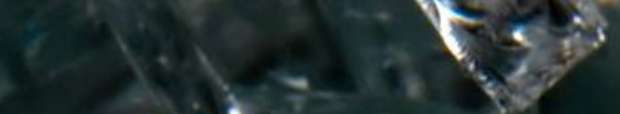

Journal of

Mechanics of

Materials and Structyres

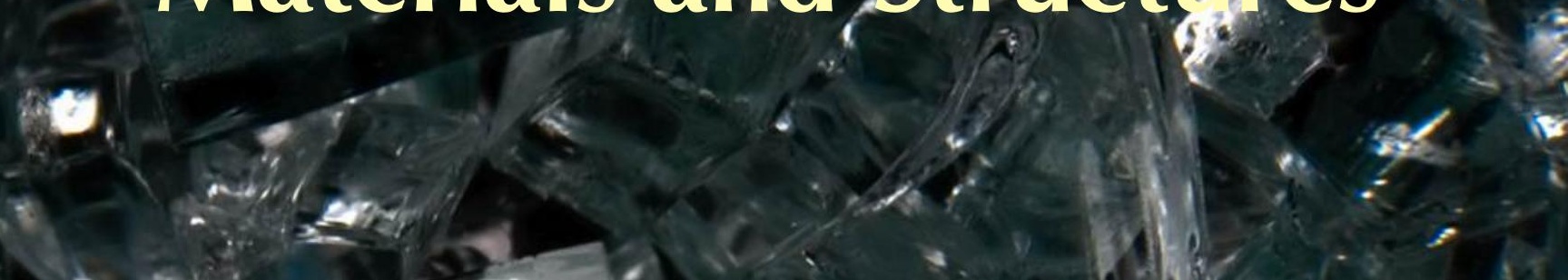

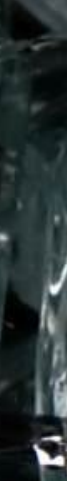

$y=1$

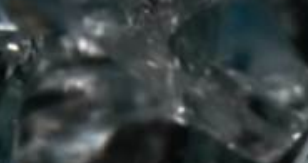

I. $2 v^{2}$

$\lim _{i \rightarrow 3} 11$

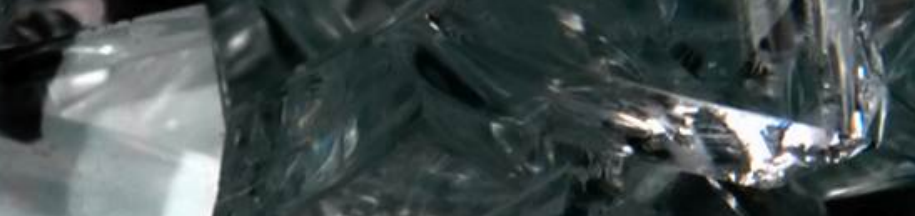

(1)

: is $2,3=0$

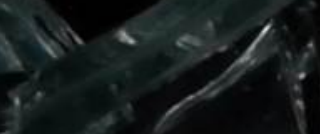
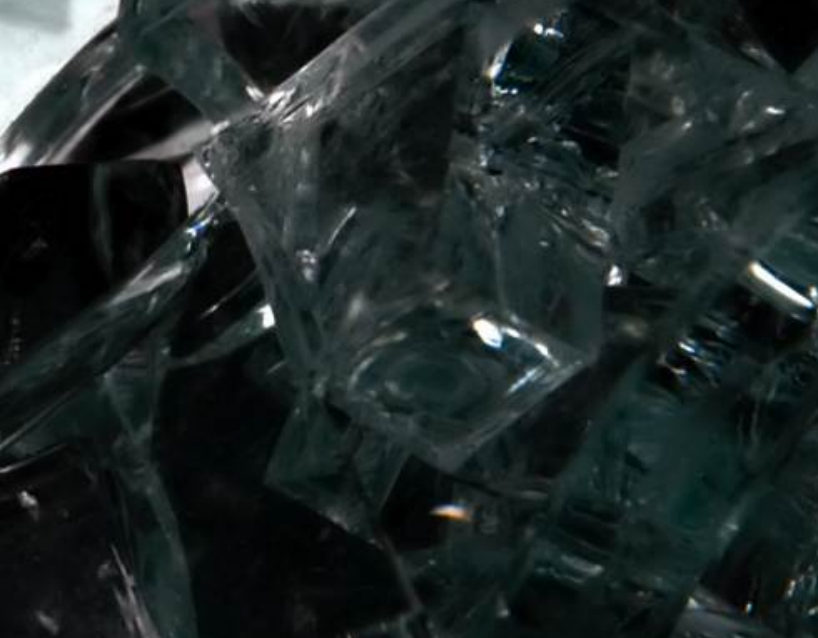

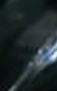

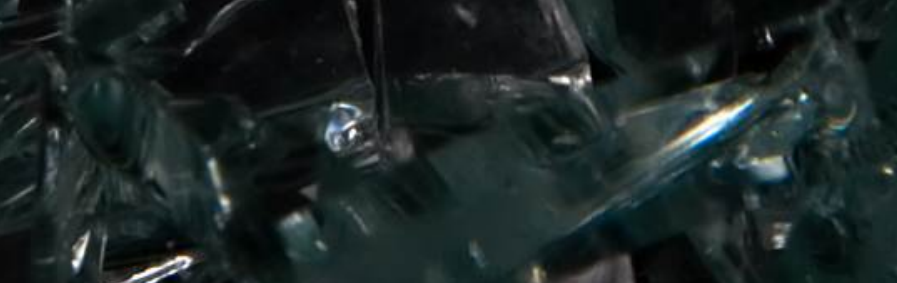

Voflume 11 , No. $20: ?$

- March 2016

N. 


\title{
JOURNAL OF MECHANICS OF MATERIALS AND STRUCTURES
}

\author{
msp.org/jomms
}

Founded by Charles R. Steele and Marie-Louise Steele

\section{EDITORIAL BOARD}

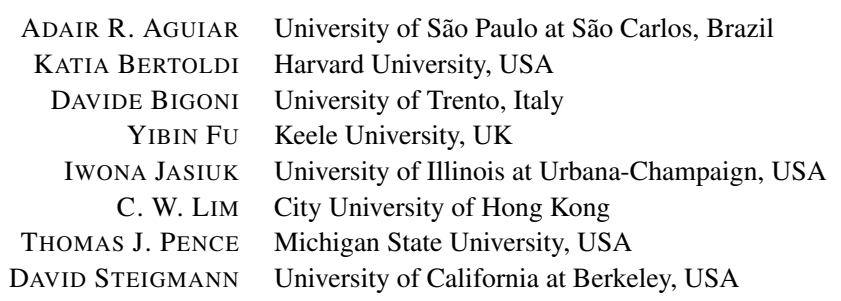

\section{ADVISORY BOARD}

J. P. CARTER University of Sydney, Australia

D. H. Hodges Georgia Institute of Technology, USA

J. HUTCHINSON Harvard University, USA

D. PAMPlona Universidade Católica do Rio de Janeiro, Brazil

M. B. RUBin Technion, Haifa, Israel

PRODUCTION production@msp.org

SILvio Levy Scientific Editor

See msp.org/jomms for submission guidelines.

JoMMS (ISSN 1559-3959) at Mathematical Sciences Publishers, 798 Evans Hall \#6840, c/o University of California, Berkeley, CA 94720-3840, is published in 10 issues a year. The subscription price for 2016 is US \$575/year for the electronic version, and $\$ 735 /$ year ( $\$ 60$, if shipping outside the US) for print and electronic. Subscriptions, requests for back issues, and changes of address should be sent to MSP.

JoMMS peer-review and production is managed by EditFLOW ${ }^{\circledR}$ from Mathematical Sciences Publishers.

PUBLISHED BY

mathematical sciences publishers

nonprofit scientific publishing

http://msp.org/

(C) 2016 Mathematical Sciences Publishers 


\title{
THE EFFECT OF SMALL SCALE ON THE FREE VIBRATION OF FUNCTIONALLY GRADED TRUNCATED CONICAL SHELLS
}

\author{
YAghoub TAdi Beni AND FAHIMEH MEHRALIAN
}

\begin{abstract}
In this paper, the FG thin truncated conical shell formulation is developed using the modified couple stress theory. The material distributions in FG conical shell are assumed to vary continuously along shell thickness according to volume fraction of constituents based on power law distribution. The governing equations and boundary conditions are derived using Hamilton's principle, and, in the special case, the free vibration of the simply supported FG conical nanoshell is investigated using Galerkin method. Finally, the effects of parameters such as dimensionless length scale parameter, apex angle, gradient index and length on the natural frequency are examined. According to the studies conducted, the modified couple stress theory predicts the stiffness of conical nanoshell with higher accuracy than the classical continuum theory. Besides, the increasing effect of the length scale parameter on increase in natural frequency caused by decrease in length and increase in circumferential and axial wave numbers is investigated as well.
\end{abstract}

\section{Introduction}

Compared to homogeneous materials, laminated composite materials are considered as structural elements in different industries because of their high stiffness-weight and strength-weight ratios and the ability of changing structural properties to meet specific needs. However, due to discontinuity existing in material properties in the interfaces between two different materials, the stress concentration resulting in cracks and delamination phenomenon has rendered the use of these materials difficult [Sahoo and Singh 2014; Xie et al. 2014; Li et al. 2014; Furlotti et al. 2014]. Today, FGMs which are made up two isotropic materials - e.g., metal and ceramics - and enjoy the benefits of both materials are in demand by many industries [Tajalli et al. 2013; Khalili et al. 2010; Shahba and Rajasekaran 2012]. The material properties of FGMs are variable continuously and smoothly along a definite direction from one surface to the next, solving the delamination problem in composite structures [Jomehzadeh et al. 2009]. The material's high toughness, low density and thermal resistance are properties that appeal to industries as varied as the biomedical, defense and aerospace industries [Kahrobaiyan et al. 2012]. Hence, it is necessary to study the behavior of FGMs in order to make a correct prediction of their static and dynamic behavior, as various studies have to date been carried out on them [Tadi Beni et al. 2015a; 2015b; Tornabene and Viola 2013; Sankar 2001; Aydogdu and Taskin 2007; Tornabene et al. 2015; Ying et al. 2008; Viola et al. 2012]. For example, using Navier's solution for the rectangular plate and finite element model based on the third order shear deformation plate theory, Reddy investigated the deformations and stresses under the influence of material distribution based on the linear third-order theory and the nonlinear first order

Keywords: free vibration, modified couple stress theory, thin shell model, functionally graded material, truncated conical shell, length scale parameter. 
theory by using von Karman's geometric nonlinearity [Reddy 2000]. Li studied the dynamic and static behaviors of the functionally graded beam by considering rotational inertia and shear deformation [Li 2008]. Using higher order shear and normal deformation theories, Kant et al. [2010] investigated the displacements and stresses of the functionally graded beams and plates and examined the analytical formulation of static behavior of simply supported functionally graded beams and plates based on higher order theories.

Today, FGMs have extensive applications in nano/micro structures such as micro/nano-electromechanical systems (MEMS/NEMS), thin films in the shape of memory alloys and atomic force microscopes (AFMs) [Craciunescu and Wuttig 2003; Fu et al. 2004; Witvrouw and Mehta 2005]. The classical continuum theory is used by most of the aforementioned studies to study micro-nanoscale devices [Yoon et al. 2005; Zhang et al. 2005; Ansari et al. 2010]. However, the results obtained in the nanoscale reveal a difference between the predictions of the results based on the classical continuum theory and the experimental results in the nanoscale. For instance, McFarland et al. observed a considerable difference between the values of stiffness obtained by the classical continuum theory and those obtained by the bending test of a polypropylene micro cantilever [McFarland and Colton 2005]. Therefore, the appropriate theory is required to correct investigation of the FGMs behavior in micro-nanodimensions. On the other hand, obtained experimental results demonstrate that mechanical properties of materials in the micro-nanoscale are size-dependent; hence, the theories should be able to correctly predict and investigate size-dependent behavior in micro-nanostructures. It should be noted that some conventional methods used in the study of micro-nanostructures are: atomistic simulations, molecular dynamics, and higher order continuum theories such as the modified couple stress theory, the strain gradient theory, and the nonlocal theory. Using these theories and by considering the size effect, researchers have to date conducted many studies showing the capabilities of these theories [Tadi Beni 2012; Tadi Beni and Abadyan 2013; Tadi Beni et al. 2012; Ke et al. 2012; Zeverdejani and Tadi Beni 2013; Abadyan et al. 2011; Sahmani and Ansari 2013; Wang et al. 2013].

The couple stress theory, as a nonclassical continuum theory containing higher order stresses is initially introduced by Toupin [1962], Mindlin and Tiersten [1962], Mindlin [1964] and Koiter [1964a; 1964b]. This theory which contains four material parameters, two lame constants and two higher order material constants is employed by researchers such as Anthoine [2000] in an attempt to investigate beam bending. Using moments of couples equilibrium equation as well as forces and moments of forces equilibrium equations, the modified couple stress theory is introduced by Yang et al. [2002]. Many studies are carried out using this theory which contains only one higher order material parameter [Roque et al. 2013; Sahmani et al. 2013; Şimşek et al. 2013; Şimşek and Reddy 2013]. For example, using the modified couple stress theory, Abbasnejad et al. [2013] examined the dynamic and static pull-in voltage of a FGM microbeam under electrostatic load and demonstrated the effect of the distribution of materials in beam's thickness on the pull-in voltage. Tadi Beni et al. [2014] employed the modified couple stress theory to examine the pull-in instability of rotational nano-mirror and to demonstrate the effect of the Casimir force on size-dependent pull-in instability. By considering nonlinear geometry and using the modified couple stress theory, Ansari et al. [2014] studied the dynamic behavior of functionally graded rectangular Mindlin microplate and discussed the effects of parameters such as length scale parameter and boundary conditions on the rectangular microplate. Based on the modified couple stress theory, Zeighampour et al. [2014b] investigated vibration and instability of double-walled carbon nanotube conveying fluid using 
Donnell's shell model. They found that the effects of parameters such as length and length scale parameter are more considerable in the modified couple stress theory than in the classical continuum theory.

Since using an appropriate model is essential for studying the behavior of structures, the investigation of the nanoscaled structure requires an appropriate model. So far, many studies are carried out to investigate the mechanical behavior of nanostructures using the beam model [Ansari et al. 2013; Mohammad Abadi and Daneshmehr 2014; Şimşek 2010]. However, it should be noted that in these investigations, nanoelements such as CNTs are also usually modeled as beams. Considering the geometry of many nanostructure elements, which are conical or cylindrical, usage the shell model will certainly results in correct prediction of the behavior of such structures. Thus, researchers are induced to investigate the mechanical and static behavior of conical shell, which is one of the appealing structural elements to many researchers due to its unique geometric properties and numerous applications [Dung et al. 2013; Sofiyev 2009; Viola et al. 2014; Su et al. 2014]. Many studies, such as [Zhao and Liew 2011; Malekzadeh and Daraie 2014], investigated the free vibration and dynamic behavior of functionally graded conical shell panels and truncated conical shell respectively, have to date conducted on conical shell. Therefore, today, considering the increasing growth of nanoscience and the high potentials of the shell model due to its unique characteristics, it is highly essential to investigate static and dynamic behaviors of the conical nanoshell. For that reason, researchers such as Zeighampour and Tadi Beni [2014a; 2015] examined the behavior of single-walled carbon nanocones (SWCNC) using the modified couple stress theory.

Therefore, this study attempts to gain a better understanding of conical nanoshells by examining the dynamic behavior of FGM conical nanoshells using the modified couple stress theory. In this paper, the equations of the FG conical nanoshell are developed using Love's thin shell theory and the modified couple stress theory, proposing considerable advantages in the modeling of nanostructures. These advantages include modeling the size effect with only one higher order material constant using the modified couple stress theory for nanoscale calculations; modeling FGMs which expands the formulation to the FG conical nanoshell and which can be extended for isotropic materials in special cases; and the ability to obtain more precise results using the shell model for structure behavior in the nanoscale. To achieve this goal, considering the above discussion, in this paper the modified couple stress theory and the thin shell model are used to investigate the free vibration of FG truncated conical nanoshell. Using the Hamilton's principle, the governing equations and the boundary conditions are derived, and, the free vibration of the simply supported FGM conical thin shell are studied in the special case using the Galerkin method. Finally, the effects of parameters such as length, dimensionless length scale parameter and apex angle on the vibration of this nanostructure are investigated as well.

\section{Governing equations}

Figure 1 illustrates an FG truncated conical shell of length $L$, radius $R_{1}$, thickness $h$, and apex angle $2 \alpha$, and the coordinate system $(x, \theta, z)$ is located on the middle surface of the conical shell.

Based on the power law distribution, the material properties of the FG conical shell are assumed to make up a mixture of metal and ceramic, are variable along shell thickness, and, based on the volume fraction of elements, these properties are considered continuously variable from the inner shell surface to outer one. The volume fraction of FG components is

$$
V_{m}=(\tilde{z} / h)^{N}, \quad V_{c}=1-V_{m},
$$




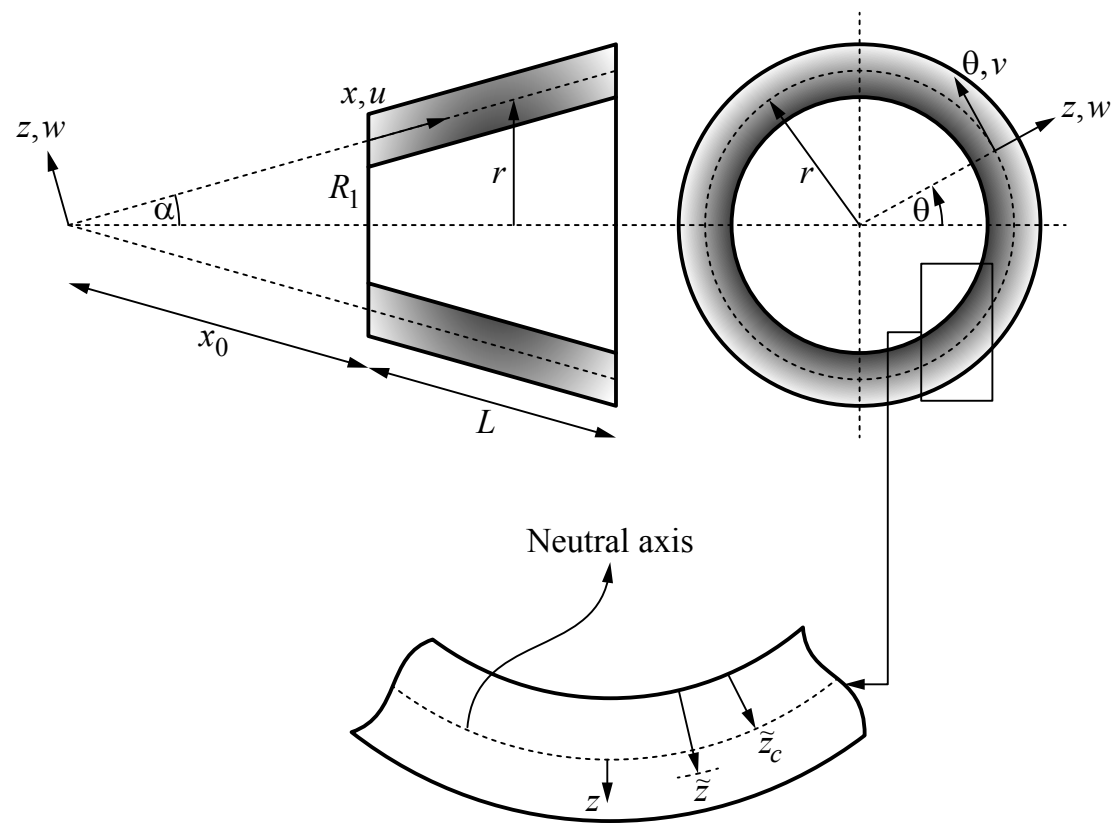

Figure 1. Schematic view of truncated conical shell.

where $N$ is defined as the power index whose variation takes place in the $0 \leq N \leq \infty$ interval and according to Figure 1, $\tilde{z}$ is the distance of an arbitrary surface from the inner one of the conical shell. Therefore, the material properties of the conical shell are defined as

$$
\begin{aligned}
& E(\tilde{z})=\left(E_{m}-E_{c}\right)\left(\frac{\tilde{z}}{h}\right)^{N}+E_{c}, \\
& \rho(\tilde{z})=\left(\rho_{m}-\rho_{c}\right)\left(\frac{\tilde{z}}{h}\right)^{N}+\rho_{c}, \\
& v(\tilde{z})=\left(v_{m}-v_{c}\right)\left(\frac{\tilde{z}}{h}\right)^{N}+v_{c},
\end{aligned}
$$

where $E_{c}, \rho_{c}$ and $v_{c}$ respectively represent Young's modulus, density, and Poisson's ratio of ceramics when $\tilde{z}=0$, and, $E_{m}, \rho_{m}$ and $v_{m}$ represent Young's modulus, density, and Poisson's ratio of metals, respectively, when $\tilde{z}=h$.

According to the modified couple stress theory introduced by Yang et al. [2002], the strain energy is expressed as a function of strain tensor and curvature tensor as

$$
U=\frac{1}{2} \int_{\Omega}(\boldsymbol{\sigma}: \boldsymbol{\epsilon}+\boldsymbol{m}: \boldsymbol{\chi}) d V,
$$

where $\boldsymbol{\epsilon}$ stands for the strain tensor, $\sigma$ represents the Cauchy stress tensor, $\chi$ is the symmetric curvature tensor and $\boldsymbol{m}$ is the deviatoric part of couple stress tensor, and, the components of the introduced tensors 
are defined as

$$
\begin{aligned}
\epsilon_{i j} & =\frac{1}{2}\left(u_{i, j}+u_{j, i}\right), \\
\chi_{i j} & =\frac{1}{4}\left(e_{i p q} \eta_{j p q}+e_{j p q} \eta_{i p q}\right), \\
\sigma_{i j} & =C_{i j k l} \epsilon_{k l}, \\
m_{i j} & =2 l^{2} \mu(\tilde{z}) \chi_{i j},
\end{aligned}
$$

where $u_{i}, e_{i p q}$ and $\eta_{i p q}$ are the components of the displacement vector, permutation symbol and deviatoric stretch gradient tensor, respectively, and $l$ stands for the material length scale parameter. It should be noted that this study is ignored the variation of parameter $l$ with material coordinates, and the value of $l$ in the whole of the FG material is considered constant.

With the assumption of plane stress, the stress-strain equations for the FGM are defined as

$$
\sigma_{x x}=\frac{E(\tilde{z})}{1-v^{2}(\tilde{z})}\left(\epsilon_{x x}+v(\tilde{z}) \epsilon_{\theta \theta}\right), \quad \sigma_{\theta \theta}=\frac{E(\tilde{z})}{1-v^{2}(\tilde{z})}\left(\epsilon_{\theta \theta}+v(\tilde{z}) \epsilon_{x x}\right), \quad \sigma_{x \theta}=2 \mu(\tilde{z}) \epsilon_{x \theta},
$$

where $\mu(\tilde{z})$ is the shear modulus and $E(\tilde{z})$ and $v(\tilde{z})$ are Young's modulus and Poisson's ratio, respectively. The displacement field of the conical shell based on Love's thin shell along $x, \theta, z$ represented respectively by $u, v, w$ is defined [Leissa 1993] as

$$
\begin{aligned}
u(x, \theta, z, t) & =U(x, \theta, t)-z \frac{\partial W(x, \theta, t)}{\partial x}, \\
v(x, \theta, z, t) & =V(x, \theta, t)-\frac{z}{x \sin \alpha}\left(\frac{\partial W(x, \theta, t)}{\partial \theta}-V(x, \theta, t) \cos \alpha\right), \\
w(x, \theta, z, t) & =W(x, \theta, t),
\end{aligned}
$$

where $U(x, \theta, t), V(x, \theta, t)$, and $W(x, \theta, t)$ are neutral surface displacement of the conical shell, and the position of this plate $\left(\tilde{z}_{c}\right)$ is determined by using the equilibrium of longitudinal forces [Barber 2011] as

$$
\int_{A} \sigma_{x x} d A=\int_{A} \frac{E(\tilde{z})}{1-v^{2}(\tilde{z})}\left(z \frac{\partial^{2} w}{\partial x^{2}}\right) d A=0,
$$

where

$$
z=\tilde{z}-\tilde{z}_{c}
$$

By substituting (11) into (10), we obtain

$$
\tilde{z}_{C}=\frac{\int_{A} \frac{E(\tilde{z})}{1-v^{2}(\tilde{z})} \tilde{z} d A}{\int_{A} \frac{E(\tilde{z})}{1-v^{2}(\tilde{z})} d A} .
$$

The classic and nonclassical strain tensors in orthogonal coordinate system are obtained by using the equations [Eringen 1980; Zhao and Pedroso 2008] 


$$
\begin{aligned}
& \epsilon_{(j)}^{(i)}=\epsilon_{j}^{i} \sqrt{\frac{g_{\underline{i i}}}{g_{\underline{j j}}}}=\frac{1}{2} \sqrt{\frac{g_{\underline{i i}}}{g_{\underline{j j}}}}\left[\left(\left(\frac{u^{(i)}}{\sqrt{g_{\underline{i i}}}}\right)_{, j}+\Gamma_{m j}^{i} \frac{u^{(m)}}{\sqrt{g_{\underline{m m}}}}\right)+g_{n j} g^{i m}\left(\left(\frac{u^{(n)}}{\sqrt{g_{\underline{n n}}}}\right)_{, m}+\Gamma_{q m}^{n} \frac{u^{(q)}}{\sqrt{g_{\underline{q q}}}}\right)\right], \\
& \eta_{(i)(j)}^{(k)}=\eta_{i j}^{k} \sqrt{\frac{g_{\underline{k k}}}{g_{\underline{i i}} g_{\underline{j} j}}}=\frac{1}{2} \sqrt{\frac{g_{\underline{k} k}}{g_{\underline{i i}} g_{\underline{j} j}}}\left(\left.u^{k}\right|_{i j}+\left.u^{k}\right|_{j i}\right), \\
& \left.u^{k}\right|_{l m}=\left(\frac{u^{(k)}}{\sqrt{g_{\underline{k k}}}}\right)_{, l m}+\Gamma_{q l}^{k}\left(\frac{u^{(q)}}{\sqrt{g_{\underline{q q}}}}\right)_{, m}+\Gamma_{q m}^{k}\left(\frac{u^{(q)}}{\sqrt{g_{\underline{q q}}}}\right)_{, l}-\Gamma_{m l}^{q}\left(\frac{u^{(k)}}{\sqrt{g_{\underline{k k}}}}\right)_{, q} \\
& +\left(\left(\Gamma_{l p}^{k}\right)_{, m}+\Gamma_{q m}^{k} \Gamma_{p l}^{q}-\Gamma_{p q}^{k} \Gamma_{m l}^{q}\right)\left(\frac{u^{(p)}}{\sqrt{g_{\underline{p p}}}}\right),
\end{aligned}
$$

where $u^{(i)}, \epsilon_{(j)}^{(i)}$ and $\eta_{(i)(j)}^{(k)}$ are the physical components of displacement vector $u^{i}$, displacement gradient $\epsilon_{i j}$ and higher-order displacement gradient $\eta_{i j}^{k}$, and $g_{i i}$ and $\Gamma_{j k}^{i}$ stand for components of the metric tensor and Christoffel symbols of the second kind. Underscores placed under the indices indicate lack of addition on them. In the cylindrical coordinate system, the components of metric tensor and Christoffel symbol are expressed as

$$
\begin{aligned}
& g_{x x}=1, \quad g_{\theta \theta}=(x \sin \alpha(1+z / x \tan \alpha))^{2}, \\
& g_{z z}=1, \quad g_{k l}=0(k \neq l), \\
& \Gamma_{z \theta}^{\theta}=\Gamma_{\theta z}^{\theta}=\frac{1}{x \tan \alpha(1+z / x \tan \alpha)}, \\
& \Gamma_{\theta \theta}^{z}=-x \sin \alpha \cos \alpha(1+z / x \tan \alpha), \\
& \Gamma_{x \theta}^{\theta}=\Gamma_{\theta x}^{\theta}=\frac{1}{x(1+z / x \tan \alpha)}, \\
& \Gamma_{\theta \theta}^{x}=-x \sin ^{2} \alpha(1+z / x \tan \alpha) .
\end{aligned}
$$

Classical strain tensors components are obtained by substituting (16) into (13) as

$$
\begin{aligned}
& \epsilon_{z z}=\frac{\partial w}{\partial z}, \quad \epsilon_{\theta \theta}=\frac{1}{x \sin \alpha(1+z / x \tan \alpha)}\left[\frac{\partial v}{\partial \theta}+u \sin \alpha+w \cos \alpha\right], \quad \epsilon_{x x}=\frac{\partial u}{\partial x}, \\
& \epsilon_{x \theta}=\epsilon_{\theta x}=\frac{1}{2 x \sin \alpha(1+z / x \tan \alpha)}\left[\frac{\partial u}{\partial \theta}+x \sin \alpha(1+z / x \tan \alpha) \frac{\partial v}{\partial x}-v \sin \alpha\right], \\
& \epsilon_{\theta z}=\epsilon_{z \theta}=\frac{1}{2 x \sin \alpha(1+z / x \tan \alpha)}\left[\frac{\partial w}{\partial \theta}+x \sin \alpha(1+z / x \tan \alpha) \frac{\partial v}{\partial z}-v \cos \alpha\right], \\
& \epsilon_{z x}=\epsilon_{x z}=\frac{1}{2}\left[\frac{\partial w}{\partial x}+\frac{\partial u}{\partial z}\right],
\end{aligned}
$$

and the components of the deviatoric stretch gradient tensor are obtained by substituting (15) and (16) into (14) as

$$
\eta_{x x x}=\frac{\partial^{2} u}{\partial x^{2}}, \quad \eta_{z z z}=\frac{\partial^{2} w}{\partial z^{2}}, \quad \eta_{x x \theta}=\frac{\partial^{2} v}{\partial x^{2}}, \quad \eta_{z z \theta}=\frac{\partial^{2} v}{\partial z^{2}}
$$


$\eta_{x \theta \theta}=\eta_{\theta x \theta}=\frac{\frac{\partial^{2} v}{\partial x \partial \theta}-\frac{1}{x(1+z / x \tan \alpha)} \frac{\partial v}{\partial \theta}-\frac{u \sin \alpha+w \cos \alpha}{x(1+z / x \tan \alpha)}+\cos \alpha \frac{\partial w}{\partial x}+\sin \alpha \frac{\partial u}{\partial x}}{x \sin \alpha(1+z / x \tan \alpha)}$,

$\eta_{z \theta \theta}=\eta_{\theta z \theta}=\frac{\frac{\partial^{2} v}{\partial z \partial \theta}-\frac{1}{x \tan \alpha(1+z / x \tan \alpha)} \frac{\partial v}{\partial \theta}-\frac{w \cos \alpha+u \sin \alpha}{x \tan \alpha(1+z / x \tan \alpha)}+\cos \alpha \frac{\partial w}{\partial z}+\sin \alpha \frac{\partial u}{\partial z}}{x \sin \alpha(1+z / x \tan \alpha)}$,

$\eta_{\theta x x}=\eta_{x \theta x}=\frac{\frac{\partial^{2} u}{\partial x \partial \theta}-\frac{1}{x(1+z / x \tan \alpha)} \frac{\partial u}{\partial \theta}-\sin \alpha \frac{\partial v}{\partial x}+\frac{v \sin \alpha}{x(1+z / x \tan \alpha)}}{x \sin \alpha(1+z / x \tan \alpha)}$,

$\eta_{\theta z z}=\eta_{z \theta z}=\frac{\frac{\partial^{2} w}{\partial z \partial \theta}-\frac{1}{x \tan \alpha(1+z / x \tan \alpha)} \frac{\partial w}{\partial \theta}-\cos \alpha \frac{\partial v}{\partial z}+\frac{v \cos \alpha}{x \tan \alpha(1+z / x \tan \alpha)}}{x \sin \alpha(1+z / x \tan \alpha)}$,

$\eta_{\theta \theta x}=\frac{\frac{\partial^{2} u}{\partial \theta^{2}}-u \sin ^{2} \alpha-w \sin \alpha \cos \alpha-2 \sin \alpha \frac{\partial v}{\partial \theta}+x \sin \alpha(1+z / x \tan \alpha)\left(\cos \alpha \frac{\partial u}{\partial z}+\sin \alpha \frac{\partial u}{\partial x}\right)}{(x \sin \alpha(1+z / x \tan \alpha))^{2}}$,

$\eta_{\theta \theta z}=\frac{\frac{\partial^{2} w}{\partial \theta^{2}}-w \cos ^{2} \alpha-u \sin \alpha \cos \alpha-2 \cos \alpha \frac{\partial v}{\partial \theta}+x \sin \alpha(1+z / x \tan \alpha)\left(\cos \alpha \frac{\partial w}{\partial z}+\sin \alpha \frac{\partial w}{\partial x}\right)}{(x \sin \alpha(1+z / x \tan \alpha))^{2}}$,

$\eta_{\theta \theta \theta}=\frac{\frac{\partial^{2} v}{\partial \theta^{2}}-v+x \sin ^{2} \alpha(1+z / x \tan \alpha) \frac{\partial v}{\partial x}+2 \sin \alpha \frac{\partial u}{\partial \theta}+2 \cos \alpha \frac{\partial w}{\partial \theta}+x \cos \alpha \sin \alpha(1+z / x \tan \alpha) \frac{\partial v}{\partial z}}{(x \sin \alpha(1+z / x \tan \alpha))^{2}}$,

$\eta_{x \theta z}=\eta_{\theta x z}=\frac{\frac{\partial^{2} w}{\partial x \partial \theta}-\frac{1}{x(1+z / x \tan \alpha)} \frac{\partial w}{\partial \theta}-\cos \alpha \frac{\partial v}{\partial x}+\frac{v \cos \alpha}{x(1+z / x \tan \alpha)}}{x \sin \alpha(1+z / x \tan \alpha)}$,

$\eta_{z \theta x}=\eta_{\theta z x}=\frac{\frac{\partial^{2} u}{\partial z \partial \theta}-\frac{1}{x \tan \alpha(1+z / x \tan \alpha)} \frac{\partial u}{\partial \theta}-\sin \alpha \frac{\partial v}{\partial z}+\frac{v \cos \alpha}{x(1+z / x \tan \alpha)}}{x \sin \alpha(1+z / x \tan \alpha)}$,

$\eta_{x x z}=\frac{\partial^{2} w}{\partial x^{2}}, \quad \eta_{z z x}=\frac{\partial^{2} u}{\partial z^{2}}, \quad \eta_{z x \theta}=\eta_{x z \theta}=\frac{\partial^{2} v}{\partial x \partial z}$.

Now, by substituting the displacement field according to (9) into (17) and (18) and using (5) and the assumptions of Love's thin shell theory as $(1 \pm z / x \tan \alpha) \approx 1$ and $(z / x \tan \alpha)^{2} \approx 0$, the classical and nonclassical nonzero strain components are respectively obtained as

$$
\begin{aligned}
\epsilon_{x x} & =\frac{\partial U}{\partial x}-z \frac{\partial^{2} W}{\partial x^{2}} \\
\epsilon_{\theta \theta} & =\frac{1}{x \sin \alpha}\left[\frac{\partial V}{\partial \theta}+U \sin \alpha+W \cos \alpha-\frac{z}{x \sin \alpha} \frac{\partial^{2} W}{\partial \theta^{2}}-z \sin \alpha \frac{\partial W}{\partial x}\right] \\
\epsilon_{x \theta} & =\epsilon_{\theta x}=\frac{1}{2 x \sin \alpha}\left[\frac{\partial U}{\partial \theta}+x \sin \alpha \frac{\partial V}{\partial x}-V \sin \alpha-2 z \frac{\partial^{2} W}{\partial x \partial \theta}+\frac{2 z}{x} \frac{\partial W}{\partial \theta}\right],
\end{aligned}
$$


and

$$
\begin{aligned}
& \chi_{z z}=\frac{1}{2 x^{2} \sin ^{2} \alpha}\left[\cos \alpha \frac{\partial U}{\partial \theta}+x \sin \alpha \cos \alpha\left[\frac{\partial V}{\partial x}-\frac{V}{x}\right]-2 z \cos \alpha \frac{\partial^{2} W}{\partial x \partial \theta}+\frac{2 z \cos \alpha}{x} \frac{\partial W}{\partial \theta}\right], \\
& \chi_{x x}=\frac{1}{x \sin \alpha}\left[\cos \alpha\left[\frac{V}{x}-\frac{\partial V}{\partial x}\right]+\frac{\partial^{2} W}{\partial x \partial \theta}-\frac{1}{x} \frac{\partial W}{\partial \theta}\right], \\
& \chi_{\theta \theta}=\frac{1}{2 x \sin \alpha}\left[\cos \alpha\left[\frac{\partial V}{\partial x}-\frac{V}{x}\right]-\frac{1}{x \tan \alpha} \frac{\partial U}{\partial \theta}-2 \frac{\partial^{2} W}{\partial x \partial \theta}+\frac{2}{x} \frac{\partial W}{\partial \theta}\right], \\
& \chi_{x \theta}=\chi_{\theta x}=\frac{1}{2 x^{2} \sin ^{2} \alpha}\left[\frac{\partial^{2} W}{\partial \theta^{2}}-x^{2} \sin ^{2} \alpha \frac{\partial^{2} W}{\partial x^{2}}+x \sin ^{2} \alpha \frac{\partial W}{\partial x}-\cos \alpha \frac{\partial V}{\partial \theta}\right], \\
& \chi_{z \theta}=\chi_{\theta z}=\frac{1}{4 x^{2} \sin ^{2} \alpha}\left[x \sin \alpha \frac{\partial^{2} V}{\partial x \partial \theta}+\sin \alpha \frac{\partial V}{\partial \theta}-\frac{\partial^{2} U}{\partial \theta^{2}}+2 x \sin \alpha \cos \alpha \frac{\partial W}{\partial x}\right], \\
& \chi_{x z}=\chi_{z x}=\frac{1}{4}\left[\frac{1}{x} \frac{\partial V}{\partial x}+\frac{\partial^{2} V}{\partial x^{2}}-\frac{V}{x^{2}}+\frac{1}{x \sin \alpha}\left[\frac{1}{x} \frac{\partial U}{\partial \theta}-\frac{\partial^{2} U}{\partial x \partial \theta}\right]\right] .
\end{aligned}
$$

Now, by substituting Equations (19) and (20) into Equations (6) and (7) and determining the components of classical and nonclassical stresses, the strain energy of the conical shell is obtained using (3) as

$$
\begin{aligned}
& U_{s}=\frac{1}{2} \int_{0}^{2 \pi} \int_{x_{0}}^{x_{0}+L}\left[\left[N_{x x}\right] \frac{\partial U}{\partial x}+\left[\frac{N_{x \theta}}{x \sin \alpha}-\frac{Y_{\theta \theta} \cos \alpha}{2 x^{2} \sin ^{2} \alpha}+\frac{Y_{z z} \cos \alpha}{2 x^{2} \sin ^{2} \alpha}+\frac{Y_{x z}}{2 x^{2} \sin \alpha}\right] \frac{\partial U}{\partial \theta}\right. \\
&-\left[\frac{Y_{z \theta}}{2 x^{2} \sin ^{2} \alpha}\right] \frac{\partial^{2} U}{\partial \theta^{2}}-\left[\frac{Y_{z x}}{2 x \sin \alpha}\right] \frac{\partial^{2} U}{\partial x \partial \theta}+\left[\frac{N_{\theta \theta}}{x}-\frac{N_{x x}}{x}\right] U+\left[\frac{Y_{z \theta}}{2 x \sin \alpha}\right] \frac{\partial^{2} V}{\partial x \partial \theta} \\
&+ {\left[\frac{Y_{z x}}{2}\right] \frac{\partial^{2} V}{\partial x^{2}}+\left[N_{x \theta}-\frac{Y_{x x}}{x \tan \alpha}+\frac{Y_{z z}}{2 x \tan \alpha}+\frac{Y_{\theta \theta}}{2 x \tan \alpha}+\frac{Y_{x z}}{2 x}\right] \frac{\partial V}{\partial x} } \\
&+ {\left[\frac{N_{\theta \theta}}{x \sin \alpha}-\frac{Y_{x \theta} \cos \alpha}{x^{2} \sin ^{2} \alpha}+\frac{Y_{z \theta}}{2 x^{2} \sin \alpha}\right] \frac{\partial V}{\partial \theta} } \\
&+ {\left[-\frac{N_{x \theta}}{x}+\frac{Y_{x x}}{x^{2} \tan \alpha}-\frac{Y_{z z}}{2 x^{2} \tan \alpha}-\frac{Y_{\theta \theta}}{2 x^{2} \tan \alpha}-\frac{Y_{x z}}{2 x^{2}}\right] V } \\
&+ {\left[\frac{2 M_{x x}}{x}-\frac{M_{\theta \theta}}{x}+\frac{Y_{z \theta}}{x \tan \alpha}+\frac{Y_{x \theta}}{2 x}\right] \frac{\partial W}{\partial x}-\left[M_{x x}+Y_{x \theta}\right] \frac{\partial^{2} W}{\partial x^{2}}+\left[\frac{N_{\theta \theta}}{x \tan \alpha}\right] W } \\
&-\left[\frac{M_{\theta \theta}}{x^{2} \sin ^{2} \alpha}-\frac{Y_{x \theta}}{x^{2} \sin ^{2} \alpha}\right] \frac{\partial^{2} W}{\partial \theta^{2}}+\left[\frac{2 M_{x \theta}}{x^{2} \sin \alpha}-\frac{Y_{x x}}{x^{2} \sin \alpha}+\frac{Y_{\theta \theta}}{x^{2} \sin \alpha}+\frac{T_{z z} \cos \alpha}{x^{3} \sin 2}\right] \frac{\partial W}{\partial \theta} \\
&\left.-\left[\frac{2 M_{x \theta}}{x \sin \alpha}-\frac{Y_{x x}}{x \sin \alpha}+\frac{Y_{\theta \theta}}{x \sin \alpha}+\frac{T_{z z} \cos \alpha}{x^{2} \sin ^{2} \alpha}\right] \frac{\partial^{2} W}{\partial x \partial \theta}\right] x \sin \alpha d x d \theta,
\end{aligned}
$$


where the classical and nonclassical force and momentum are expressed as

$$
N_{i j}=\int_{-\tilde{z}_{c}}^{h-\tilde{z}_{c}} \sigma_{i j} d z, \quad M_{i j}=\int_{-\tilde{z}_{c}}^{h-\tilde{z}_{c}} \sigma_{i j} z d z, \quad Y_{i j}=\int_{-\tilde{z}_{c}}^{h-\tilde{z}_{c}} m_{i j} d z, \quad T_{i j}=\int_{-\tilde{z}_{c}}^{h-\tilde{z}_{c}} m_{i j} z d z
$$

and the kinetic energy of the conical thin shell is defined as

$$
T=\frac{1}{2} \int_{\Omega} \rho(\tilde{z})\left[\left(\frac{\partial U}{\partial t}\right)^{2}+\left(\frac{\partial V}{\partial t}\right)^{2}+\left(\frac{\partial W}{\partial t}\right)^{2}\right] x \sin \alpha d x d \theta d z
$$

where $\rho(\tilde{z})$ stands for the density of the conical shell.

Also, the work done by external forces acting on the conical shell is expressed as

$$
\begin{aligned}
w_{e} & =w_{d}+w_{b}, \\
w_{d} & =\int_{\theta} \int_{x}\left(f_{x} U+f_{\theta} V+f_{z} W\right) x \sin \alpha d x d \theta, \\
w_{b} & =\left.\int_{\theta}\left[\bar{N}_{x}^{u} U+\bar{N}_{x}^{v} V+\bar{N}_{x}^{w} W+\hat{P}_{x}^{v h} \frac{\partial V}{\partial x}+\bar{M}_{x}^{w} \frac{\partial W}{\partial x}\right] x \sin \alpha d \theta\right|_{x_{0}} ^{x_{0}+L}, \\
& +\left.\int_{x}\left[\bar{N}_{\theta}^{u} U+\bar{N}_{\theta}^{v} V+\bar{N}_{\theta}^{w} W+\hat{P}_{\theta}^{u h} \frac{\partial U}{\partial \theta}+\bar{M}_{\theta}^{w} \frac{\partial W}{\partial \theta}\right] x \sin \alpha d x\right|_{0} ^{\theta_{0}},
\end{aligned}
$$

where $f_{x}, f_{\theta}$ and $f_{z}$ represent volume distributed forces and $\bar{N}_{x}^{u}, \bar{N}_{x}^{v}, \bar{N}_{x}^{w}, \hat{P}_{x}^{v h}, \bar{M}_{x}^{w}, \bar{N}_{\theta}^{u}, \bar{N}_{\theta}^{v}, \bar{N}_{\theta}^{w}$, $\hat{P}_{\theta}^{u h}, \bar{M}_{\theta}^{w}$ are the classical and nonclassical force and momentum applied to typical $(x=$ constant $)$ and $(\theta=$ constant $)$ lines and edge.

Now, the Hamilton's principle is

$$
\int_{t_{1}}^{t_{2}}\left(\delta T-\delta U_{s}+\delta W_{e}\right) d t=0
$$

By substituting the work done by external forces applied as well as strain energy and kinetic energy of the shell in Hamilton's principle, and using variations method and performing direct, lengthy mathematical calculations, the governing equations and boundary conditions are derived as

$$
\begin{aligned}
\delta U: & -\frac{D_{1,0}}{x} \frac{\partial U}{\partial x}+\frac{D_{1,0}}{x^{2}} U-D_{1,0} \frac{\partial^{2} U}{\partial x^{2}}+\frac{D_{5,0}}{x^{2} \sin ^{2} \alpha}\left[-1+\frac{l^{2}}{4 x^{2}}-\frac{l^{2}}{x^{2} \tan ^{2} \alpha}\right] \frac{\partial^{2} U}{\partial \theta^{2}}-\frac{D_{5,0} l^{2}}{4 x^{3} \sin ^{2} \alpha} \frac{\partial^{3} U}{\partial x \partial \theta^{2}} \\
& +\frac{D_{5,0} l^{2}}{4 x^{4} \sin ^{4} \alpha} \frac{\partial^{4} U}{\partial \theta^{4}}+\frac{D_{5,0} l^{2}}{4 x^{2} \sin ^{2} \alpha} \frac{\partial^{4} U}{\partial x^{2} \partial \theta^{2}}+\frac{1}{x^{2} \sin \alpha}\left[D_{1,0}+D_{5,0}\left[1-\frac{l^{2}}{4 x^{2}}\right]\right] \frac{\partial V}{\partial \theta}-\frac{D_{5,0} l^{2}}{2 x^{2} \sin \alpha} \frac{\partial^{3} V}{\partial x^{2} \partial \theta} \\
& -\frac{1}{x \sin \alpha}\left[D_{3,0}+D_{5,0}\left[1-\frac{l^{2}}{4 x^{2}}\right]\right] \frac{\partial^{2} V}{\partial x \partial \theta}-\frac{D_{5,0} l^{2}}{4 x^{3} \sin ^{3} \alpha} \frac{\partial^{4} V}{\partial x}-\frac{D_{5,0} l^{2}}{4 x \sin \alpha} \frac{\partial^{4} V}{\partial x^{3} \partial \theta}-\frac{D_{5,0} l^{2}}{4 x^{4} \sin ^{3} \alpha} \frac{\partial^{3} V}{\partial \theta^{3}} \\
& +\frac{1}{x^{3} \sin ^{2} \alpha}\left[\frac{D_{5,0} l^{2}}{x \tan \alpha}-D_{1,1}-2 D_{5,1}-\frac{D_{5,1} l^{2}}{x^{2} \tan ^{2} \alpha}-D_{3,1}\right] \frac{\partial^{2} W}{\partial \theta^{2}}+\frac{D_{1,0}}{x^{2} \tan \alpha} W-\frac{1}{x}\left[\frac{D_{3,0}}{\tan \alpha}+\frac{D_{1,1}}{x}\right] \frac{\partial W}{\partial x} \\
& -\frac{1}{x^{2} \sin ^{2} \alpha}\left[\frac{3 D_{5,0} l^{2}}{2 x \tan \alpha}-D_{3,1}-2 D_{5,1}-\frac{D_{5,1} l^{2}}{x^{2} \tan ^{2} \alpha}\right] \frac{\partial^{3} W}{\partial x \theta^{2}}+D_{1,1} \frac{\partial^{3} W}{\partial x^{3}}+\frac{D_{1,1}}{x} \frac{\partial^{2} W}{\partial x^{2}}-f_{x}+I_{1,0} \frac{\partial^{2} U}{\partial t^{2}}=0
\end{aligned}
$$




$$
\begin{aligned}
\delta V: & -\frac{1}{x^{2} \sin \alpha}\left[D_{1,0}+D_{5,0}\left[1-\frac{3 l^{2}}{4 x^{2}}\right]\right] \frac{\partial U}{\partial \theta}-\frac{1}{x \sin \alpha}\left[D_{3,0}+D_{5,0}\left[1+\frac{3 l^{2}}{4 x^{2}}\right]\right] \frac{\partial^{2} U}{\partial x \partial \theta} \\
& +\frac{D_{5,0} l^{2}}{2 x^{2} \sin \alpha} \frac{\partial^{3} U}{\partial x^{2} \partial \theta}+\frac{3 D_{5,0} l^{2}}{4 x^{4} \sin ^{3} \alpha} \frac{\partial^{3} U}{\partial \theta^{3}}-\frac{D_{5,0} l^{2}}{4 x \sin \alpha} \frac{\partial^{4} U}{\partial x^{3} \partial \theta}-\frac{D_{5,0} l^{2}}{4 x^{3} \sin ^{3} \alpha} \frac{\partial^{4} U}{\partial x \partial \theta^{3}} \\
& +\frac{D_{5,0}}{x^{2}}\left[1-\frac{3 l^{2}}{x^{2} \tan ^{2} \alpha}-\frac{3 l^{2}}{4 x^{2}}\right] V-\frac{D_{5,0}}{x}\left[1-\frac{3 l^{2}}{x^{2} \tan ^{2} \alpha}-\frac{3 l^{2}}{4 x^{2}}\right] \frac{\partial V}{\partial x}+\frac{D_{5,0} l^{2}}{2 x} \frac{\partial^{3} V}{\partial x^{3}} \\
& -D_{5,0}\left[1+\frac{3 l^{2}}{x^{2} \tan ^{2} \alpha}+\frac{3 l^{2}}{4 x^{2}}\right] \frac{\partial^{2} V}{\partial x^{2}}+\frac{D_{5,0} l^{2}}{4} \frac{\partial^{4} V}{\partial x^{4}}-\frac{1}{x^{2} \sin ^{2} \alpha}\left[D_{1,0}+\frac{D_{5,0} l^{2}}{x^{2}}\left[\frac{1}{\tan ^{2} \alpha}+\frac{3}{4}\right]\right] \frac{\partial^{2} V}{\partial \theta^{2}} \\
& -\frac{D_{5,0} l^{2}}{4 x^{3} \sin ^{2} \alpha} \frac{\partial^{3} V}{\partial x \partial \theta^{2}}+\frac{D_{5,0} l^{2}}{4 x^{2} \sin ^{2} \alpha} \frac{\partial^{4} V}{\partial x^{2} \partial \theta^{2}}-\frac{1}{x^{2} \sin \alpha}\left[\frac{3 D_{5,0} l^{2}}{x \tan \alpha}-D_{1,1}+\frac{2 D_{5,1} l^{2}}{x^{2} \tan ^{2} \alpha}\right] \frac{\partial^{2} W}{\partial x \partial \theta} \\
& +\frac{1}{x^{3} \sin ^{3} \alpha}\left[\frac{D_{5,0} l^{2}}{x \tan \alpha}+D_{1,1}\right] \frac{\partial^{3} W}{\partial \theta^{3}}-\frac{1}{x^{2} \sin ^{2} \alpha}\left[D_{1,0} \cos \alpha-\frac{3 D_{5,0} l^{2} \cos \alpha}{x^{2}}\right] \frac{\partial W}{\partial \theta} \\
& +\frac{1}{x \sin ^{2}}\left[\frac{5 D_{5,0} l^{2}}{2 x \tan \alpha}+D_{3,1}+2 D_{5,1}+\frac{D_{5,1} l^{2}}{x^{2} \tan ^{2} \alpha}\right] \frac{\partial^{3} W}{\partial x^{2} \partial \theta}-f_{\theta}+I_{1,0} \frac{\partial^{2} V}{\partial t^{2}}=0,
\end{aligned}
$$

$\delta W: \frac{1}{x}\left[\frac{D_{3,0}}{\tan \alpha}+\frac{D_{1,1}}{x}\right] \frac{\partial U}{\partial x}+\frac{1}{x^{2}}\left[\frac{D_{1,0}}{\tan \alpha}-\frac{D_{1,1}}{x}\right] U-\frac{1}{x^{3} \sin ^{2} \alpha}\left[\frac{2 D_{5,0} l^{2}}{x \tan \alpha}-\frac{2 D_{5,1} l^{2}}{x^{2} \tan ^{2} \alpha}+D_{1,1}\right] \frac{\partial^{2} U}{\partial \theta^{2}}$

$$
\begin{aligned}
& +\frac{1}{x^{2} \sin ^{2} \alpha}\left[\frac{3 D_{5,0} l^{2}}{2 x \tan \alpha}-\frac{D_{5,1} l^{2}}{x^{2} \tan ^{2} \alpha}-D_{3,1}-2 D_{5,1}\right] \frac{\partial^{3} U}{\partial x \partial \theta^{2}}-D_{1,1} \frac{\partial^{3} U}{\partial x^{3}}-\frac{2 D_{1,1}}{x} \frac{\partial^{2} U}{\partial x^{2}} \\
& +\frac{1}{x^{2} \sin \alpha}\left[\frac{D_{1,0}}{\tan \alpha}-\frac{2 D_{5,0} l^{2}}{x^{2} \tan \alpha}+\frac{2 D_{5,1}}{x}-\frac{2 D_{5,1} l^{2}}{x^{3} \tan ^{2} \alpha}-\frac{D_{1,1}}{x}-\frac{2 D_{3,1}}{x}\right] \frac{\partial V}{\partial \theta}-\frac{1}{x \sin \alpha}\left[\frac{5 D_{5,0} l^{2}}{2 x \tan \alpha}\right.
\end{aligned}
$$$$
\left.+\frac{D_{5,1} l^{2}}{x^{2} \tan ^{2} \alpha}+D_{3,1}+2 D_{5,1}\right] \frac{\partial^{3} V}{\partial x^{2} \partial \theta}-\frac{1}{x^{3} \sin ^{3} \alpha}\left[\frac{D_{5,0} l^{2}}{x \tan \alpha}+D_{1,1}\right] \frac{\partial^{3} V}{\partial \theta^{3}}
$$$$
+\frac{1}{x^{2} \sin \alpha}\left[\frac{2 D_{5,0} l^{2}}{x \tan \alpha}+\frac{2 D_{5,1} l^{2}}{x^{2} \tan ^{2} \alpha}+D_{1,1}\right] \frac{\partial^{2} V}{\partial x \partial \theta}+\frac{1}{x^{2} \tan \alpha}\left[\frac{D_{1,0}}{\tan \alpha}-\frac{D_{1,1}}{x}\right] W
$$$$
+\frac{1}{x^{2} \sin ^{2} \alpha}\left[\frac{2 D_{5,2} l^{2}}{x^{2} \tan ^{2} \alpha}+2 D_{3,2}+4 D_{5,2}+2 D_{5,0} l^{2}\right] \frac{\partial^{4} W}{\partial x^{2} \partial \theta^{2}}+\frac{2}{x}\left[D_{1,2}+D_{5,0} l^{2}\right] \frac{\partial^{3} W}{\partial x^{3}}
$$$$
+\frac{1}{x^{3} \sin ^{2} \alpha}\left[\frac{2 D_{1,2}}{x}+\frac{2 D_{3,2}}{x}+\frac{4 D_{5,2}}{x}+\frac{4 D_{5,0} l^{2}}{x}+\frac{6 D_{5,2} l^{2}}{x^{3} \tan ^{2} \alpha}-\frac{2 D_{1,1}}{\tan \alpha}\right] \frac{\partial^{2} W}{\partial \theta^{2}}
$$$$
-\frac{1}{x^{3} \sin ^{2} \alpha}\left[\frac{6 D_{5,2} l^{2}}{x^{2} \tan ^{2} \alpha}+2 D_{3,2}+4 D_{5,2}+2 D_{5,0} l^{2}\right] \frac{\partial^{3} W}{\partial x \partial \theta^{2}}+\frac{1}{x^{3}}\left[D_{1,2}+D_{5,0} l^{2}\left[1+\frac{1}{\tan ^{2} \alpha}\right]\right] \frac{\partial W}{\partial x}
$$$$
-\frac{1}{x}\left[\frac{D_{1,2}}{x}+\frac{D_{5,0} l^{2}}{x}\left[1+\frac{1}{\tan ^{2} \alpha}\right]+\frac{2 D_{3,1}}{\tan \alpha}\right] \frac{\partial^{2} W}{\partial x^{2}}+\left[D_{1,2}+D_{5,0} l^{2}\right] \frac{\partial^{4} W}{\partial x^{4}}
$$$$
+\frac{1}{x^{4} \sin ^{4} \alpha}\left[D_{1,2}+D_{5,0} l^{2}\right] \frac{\partial^{4} W}{\partial \theta^{4}}-f_{w}+I_{1,0} \frac{\partial^{2} W}{\partial t^{2}}=0
$$ 
And the boundary conditions for $x=$ constant are obtained as

$$
\begin{gathered}
\left.\int_{\theta}\left[N_{x x}+\frac{\partial}{\partial \theta}\left[\frac{Y_{z x}}{2 x \sin \alpha}\right]+\bar{N}_{x}^{u}\right] x \sin \alpha d \theta\right|_{x}=0 \quad \text { or }\left.\quad \delta U\right|_{x}=0, \\
\int_{\theta}\left[\left[N_{x \theta}-\frac{Y_{x x}}{x \tan \alpha}+\frac{Y_{z z}}{2 x \tan \alpha}+\frac{Y_{\theta \theta}}{2 x \tan \alpha}+\frac{Y_{x z}}{2 x}\right]\right. \\
\left.-\frac{\partial}{\partial \theta}\left[\frac{Y_{z \theta}}{2 x \sin \alpha}\right]-\frac{1}{x} \frac{\partial}{\partial x}\left[\frac{x Y_{z x}}{2}\right]+\bar{N}_{x}^{v}\right]\left.x \sin \alpha d \theta\right|_{x}=0 \quad \text { or }\left.\quad \delta V\right|_{x}=0, \\
\left.\int_{\theta}\left[\left[\frac{Y_{z x}}{2}\right]+\hat{P}_{x}^{v h}\right] x \sin \alpha d \theta\right|_{x}=0 \quad \text { or }\left.\delta\left(\frac{\partial V}{\partial x}\right)\right|_{x}=0, \\
\int_{\theta}\left[\frac{1}{x} \frac{\partial}{\partial x}\left[x M_{x x}+x Y_{x \theta}\right]+\left[\frac{2 M_{x x}}{x}-\frac{M_{\theta \theta}}{x}+\frac{Y_{z \theta}}{x \tan \alpha}+\frac{Y_{x \theta}}{2 x}\right]\right. \\
\left.+\frac{\partial}{\partial \theta}\left[\frac{2 M_{x \theta}}{x \sin \alpha}-\frac{Y_{x x}}{x \sin \alpha}+\frac{Y_{\theta \theta}}{x \sin \alpha}+\frac{T_{z z} \cos \alpha}{x^{2} \sin ^{2} \alpha}\right]+\bar{N}_{x}^{w}\right]\left.x \sin \alpha d \theta\right|_{x}=0 \quad \text { or }\left.\quad \delta W\right|_{x}=0, \\
\left.\int_{\theta}\left[-\left[M_{x x}+Y_{x \theta}\right]+\bar{M}_{x}^{w}\right] d \theta\right|_{x}=0 \quad \text { or }\left.\delta\left(\frac{\partial W}{\partial x}\right)\right|_{x}=0 .
\end{gathered}
$$

The boundary conditions for $\theta=$ constant are

$$
\begin{aligned}
& \int_{x}\left[\left[\frac{N_{x \theta}}{x \sin \alpha}-\frac{Y_{\theta \theta} \cos \alpha}{2 x^{2} \sin ^{2} \alpha}+\frac{Y_{z z} \cos \alpha}{2 x^{2} \sin ^{2} \alpha}+\frac{Y_{x z}}{2 x^{2} \sin \alpha}\right]\right. \\
& \left.+\frac{\partial}{\partial \theta}\left[\frac{Y_{z \theta}}{2 x^{2} \sin ^{2} \alpha}\right]+\frac{1}{x} \frac{\partial}{\partial x}\left[\frac{Y_{z x}}{2 \sin \alpha}\right]+\bar{N}_{\theta}^{u}\right]\left.x \sin \alpha d x\right|_{\theta}=0 \quad \text { or }\left.\quad \delta U\right|_{\theta}=0 \\
& \left.\int_{x}\left[-\left[\frac{Y_{z \theta}}{2 x^{2} \sin ^{2} \alpha}\right]+\hat{P}_{\theta}^{u h}\right] x \sin \alpha d x\right|_{\theta}=0 \quad \text { or }\left.\quad \delta\left(\frac{\partial U}{\partial \theta}\right)\right|_{\theta}=0 \\
& \left.\int_{x}\left[\left[\frac{N_{\theta \theta}}{x \sin \alpha}-\frac{Y_{x \theta} \cos \alpha}{x^{2} \sin ^{2} \alpha}+\frac{Y_{z \theta}}{2 x^{2} \sin \alpha}\right]-\frac{1}{x} \frac{\partial}{\partial x}\left[\frac{Y_{z \theta}}{2 \sin \alpha}\right]+\bar{N}_{\theta}^{v}\right] x \sin \alpha d x\right|_{\theta}=0 \quad \text { or }\left.\quad \delta V\right|_{\theta}=0, \\
& \int_{x}\left[\left[\frac{2 M_{x \theta}}{x^{2} \sin \alpha}-\frac{Y_{x x}}{x^{2} \sin \alpha}+\frac{Y_{\theta \theta}}{x^{2} \sin \alpha}+\frac{T_{z z} \cos \alpha}{x^{3} \sin ^{2} \alpha}\right]+\frac{1}{x} \frac{\partial}{\partial x}\left[\frac{2 M_{x \theta}}{\sin \alpha}-\frac{Y_{x x}}{\sin \alpha}+\frac{Y_{\theta \theta}}{\sin \alpha}+\frac{T_{z z} \cos \alpha}{x \sin ^{2} \alpha}\right]\right. \\
& \left.+\frac{\partial}{\partial \theta}\left[\frac{M_{\theta \theta}}{x^{2} \sin ^{2} \alpha}-\frac{Y_{x \theta}}{x^{2} \sin ^{2} \alpha}\right]+\bar{N}_{\theta}^{w}\right]\left.x \sin \alpha d x\right|_{\theta}=0 \quad \text { or }\left.\quad \delta W\right|_{\theta}=0 \\
& \left.\int_{x}\left[-\left[\frac{M_{\theta \theta}}{x^{2} \sin ^{2} \alpha}-\frac{Y_{x \theta}}{x^{2} \sin ^{2} \alpha}\right]+\bar{M}_{\theta}^{w}\right] x \sin \alpha d x\right|_{\theta}=0 \quad \text { or }\left.\quad \delta\left(\frac{\partial W}{\partial \theta}\right)\right|_{\theta}=0 .
\end{aligned}
$$


In the equations above (equations of motion and boundary conditions) parameters $D_{1, i}, D_{3, i}, D_{5, i}$ and $I_{1, i}$ are defined as

$$
\begin{aligned}
& \left(D_{1, i}\right)=\int_{-\tilde{z}_{c}}^{h-\tilde{z}_{c}} \frac{E(\tilde{z})}{1-v^{2}(\tilde{z})}\left(z^{i}\right) d z,(i=0,1,2), \\
& \left(D_{3, i}\right)=\int_{-\tilde{z}_{c}}^{h-\tilde{z}_{c}} \frac{E(\tilde{z}) v(\widehat{z})}{1-v^{2}(\tilde{z})}\left(z^{i}\right) d z,(i=0,1,2), \\
& \left(D_{5, i}\right)=\int_{-\tilde{z}_{c}}^{h-\tilde{z}_{c}} \mu(\tilde{z})\left(z^{i}\right) d z,(i=0,1,2), \\
& \left(I_{1, i}\right)=\int_{-\tilde{z}_{c}}^{h-\tilde{z}_{c}} \rho(\tilde{z})\left(z^{i}\right) d z,(i=0,1,2) .
\end{aligned}
$$

Now, it can be argued that (28)-(30) are the equations of motion of the FG conical shell based on the modified couple stress theory. Also, (31)-(40) are the classical and nonclassical boundary conditions of the FG conical shell based on the modified couple stress theory. In special cases, these equations of motion and boundary conditions are reduced to the following equations:

Case 1: By setting the length scale parameter to zero $(l=0)$, the equations obtained in this paper are reduced to the equations of motion and boundary conditions in the classical continuum theory based on the thin shell model for FG materials.

Case 2: By assuming constant mechanical properties (Young's modulus, Poisson's ratio and density), the equations obtained in this paper are reduced to the equations of motion and classical and nonclassical boundary conditions based on the modified couple stress theory and the thin shell model for the isotropic homogeneous structure.

Case 3: By assuming cases (1) and (2), the equations obtained in this paper are reduced to the equations of motion and boundary conditions in the classical continuum theory based on the thin shell model.

\section{Case study}

In this section, the free vibration of the simply supported FG conical shell in the special case is examined so as to evaluate the equations derived. The equations governing the simply supported FG conical shell are similar to (28)-(30); hence, it suffices to investigate the boundary conditions. It should be noted that the boundary conditions in (36)-(40) are satisfied due to the variability of the $\theta$ angle from 0 to $2 \pi$ edges; thus, the boundary conditions in $x=x_{0}$ and $x=x_{0}+L$ edges must be investigated, which are (31)-(35). By substituting (22) into (31)-(35), the boundary conditions for the simply supported FG conical shell are obtained as

$$
\begin{aligned}
\left.V\right|_{x=x_{0}, x_{0}+L} & =0, \\
\left.W\right|_{x=x_{0}, x_{0}+L} & =0,
\end{aligned}
$$




$$
\begin{aligned}
& {\left[D_{1,0} \frac{\partial U}{\partial x}-D_{1,1} \frac{\partial^{2} W}{\partial x^{2}}+\frac{D_{3,0}}{x} U-\frac{D_{3,1}}{x^{2} \sin ^{2} \alpha} \frac{\partial^{2} W}{\partial \theta^{2}}-\frac{D_{3,1}}{x} \frac{\partial W}{\partial x}-\frac{D_{5,0} l^{2}}{4 x^{2} \sin ^{2} \alpha} \frac{\partial^{3} U}{\partial x \partial \theta^{2}}\right.} \\
& \quad+\frac{D_{5,0} l^{2}}{4 x^{3} \sin ^{2} \alpha} \frac{\partial^{2} U}{\partial \theta^{2}}+\frac{1}{x \sin \alpha}\left[D_{3,0}-\frac{D_{5,0} l^{2}}{4 x^{2}}\right] \frac{\partial V}{\partial \theta}+\frac{D_{5,0} l^{2}}{4 x \sin \alpha} \frac{\partial^{3} V}{\partial x^{2} \partial \theta}+\frac{D_{5,0} l^{2}}{4 x^{2} \sin \alpha} \frac{\partial^{2} V}{\partial x \partial \theta} \\
& {\left.\left[-\frac{D_{5,0} l^{2}}{4 x \sin \alpha} \frac{\partial^{2} U}{\partial x}+\frac{D_{5,0} l^{2}}{4 x^{2} \sin \alpha} \frac{\partial U}{\partial \theta}+\frac{D_{5,0} l^{2}}{4} \frac{\partial^{2} V}{\partial x^{2}}+\frac{D_{5,0} l^{2}}{4 x} \frac{\partial V}{\partial x}-\frac{D_{5,0} l^{2}}{4 x^{2}} V\right]\right|_{x=x_{0}, x_{0}+L}=0} \\
& {\left[\left[D_{1,2}+D_{5,0} l^{2}\right] \frac{\partial^{2} W}{\partial x^{2}}+\frac{1}{x}\left[D_{3,2}-D_{5,0} l^{2}\right] \frac{\partial W}{\partial x}+\frac{1}{x^{2} \sin ^{2} \alpha}\left[D_{3,2}-D_{5,0} l^{2}\right] \frac{\partial^{2} W}{\partial \theta^{2}}\right.} \\
& \left.-D_{1,1} \frac{\partial U}{\partial x}+\frac{1}{x \sin \alpha}\left[\frac{D_{5,0} l^{2}}{x \tan \alpha}-D_{3,1}\right] \frac{\partial V}{\partial \theta}-\frac{D_{3,1}}{x} U-\frac{D_{3,1}}{x \tan \alpha} W\right]\left.\right|_{x=x_{0}, x_{0}+L}=0
\end{aligned}
$$

Hence, (28)-(30) as well as (42)-(45) are the governing equations and boundary conditions for the simply supported FG thin conical shell, which must be simultaneously solved in order to investigate the free vibration.

In order to solve the above equations and considering boundary conditions, the following approximate solutions are used [Dung et al. 2014]:

$$
\begin{gathered}
U(x, \theta, t)=U_{0} \cos \left(\frac{m \pi\left(x-x_{0}\right)}{L}\right) \sin (n \theta) \sin (\omega t), \quad V(x, \theta, t)=U_{0} \sin \left(\frac{m \pi\left(x-x_{0}\right)}{L}\right) \cos (n \theta) \sin (\omega t), \\
W(x, \theta, t)=W_{0} \sin \left(\frac{m \pi\left(x-x_{0}\right)}{L}\right) \sin (n \theta) \sin (\omega t) .
\end{gathered}
$$

In the above equations $\omega, n$ and $m$ stand for the natural frequency of the nanoshell, circumferential and axial wave numbers, respectively. Given the above assumption, the majority of boundary conditions in (42)-(45) are satisfied although some of them are not fully satisfied. However, in the references, it is common that for a complicated formulation, like that one above, not all boundary conditions be satisfied. Therefore, to investigate the free vibration of the nanoshell, it is necessary to solve the equations of motion in (28)-(30). For this purpose, the Galerkin method is employed. To simplify integration, (28)(29) are multiplied by $x^{4}$ and (30) is multiplied by $x^{6}$, and, finally, using the Galerkin method and the equations derived, the following equation is obtained:

$$
\begin{aligned}
& \int_{x_{0}}^{x_{0}+L} \int_{0}^{2 \pi} \psi_{1} x \cos \left(\frac{m \pi\left(x-x_{0}\right)}{L}\right) \sin \alpha d x d \theta=0, \\
& \int_{x_{0}}^{x_{0}+L} \int_{0}^{2 \pi} \psi_{2} x \sin \left(\frac{m \pi\left(x-x_{0}\right)}{L}\right) \sin \alpha d x d \theta=0, \\
& \int_{x_{0}}^{x_{0}+L} \int_{0}^{2 \pi} \psi_{3} x \sin \left(\frac{m \pi\left(x-x_{0}\right)}{L}\right) \sin \alpha d x d \theta=0,
\end{aligned}
$$




\begin{tabular}{|cccc|}
\hline & $\rho\left(\mathrm{kg} / \mathrm{m}^{3}\right)$ & $v$ & $E(\mathrm{GPa})$ \\
\hline Aluminum & 70 & 2702 & 0.3 \\
Ceramic & 427 & 3100 & 0.17 \\
\hline
\end{tabular}

Table 1. Material properties of aluminum and ceramic.

where the parameters $\psi_{1}, \psi_{2}$ and $\psi_{3}$ are obtained as

$$
\begin{aligned}
& \psi_{1}=x^{4}\left[A_{11}\left(U_{0}\right)+A_{12}\left(V_{0}\right)+A_{13}\left(W_{0}\right)\right], \\
& \psi_{2}=x^{4}\left[A_{21}\left(U_{0}\right)+A_{22}\left(V_{0}\right)+A_{23}\left(W_{0}\right)\right], \\
& \psi_{3}=x^{6}\left[A_{31}\left(U_{0}\right)+A_{32}\left(V_{0}\right)+A_{33}\left(W_{0}\right)\right] .
\end{aligned}
$$

In the above equation, $A_{i j}$ are values obtained by substituting Equation (47) into Equations (28)-(30). Hence, the matrix form of Equation (48) is

$$
\left([K]-\omega^{2}[M]\right)\left\{\begin{array}{c}
U_{0} \\
V_{0} \\
W_{0}
\end{array}\right\}=0 .
$$

Considering the eigenvalue problem, in order to obtain a nontrivial solution to Equation (49), the determinant of the coefficients must be set to zero, and, by solving the derived equation, one can determine the frequency of nanoshell.

\section{Results and discussion}

This section is devoted to the investigation of the free vibration of the simply supported FG conical thin shell using the modified couple stress theory. The effect of parameters such as dimensionless length scale parameter, apex angle, dimensionless length parameter and circumferential and axial wave numbers on the dimensionless natural frequency are studied and the results are compared with those obtained based on the classical continuum theory. As mentioned before, assuming $l=0$, the resulting equations are obtained based on the classical continuum theory.

The geometric features of the conical shell are assumed to be $h=0.34 \mathrm{~nm}, L / R_{1}=2$ and $h / R_{1}=0.04$, and the FG conical shell is assumed to consist of aluminum or ceramic with the material properties in Table 1 [Sahmani et al. 2013].

4.1. Comparison of results. Since according to the authors studies, so far, no study is carried out on the FG conical nanoshell, in order to verify the results, first the correctness of the results are shown by comparing the obtained results from the homogeneous conical shell based on the classical continuum theory and assuming $N=0$ with that of [Irie et al. 1984; Jin et al. 2014; Lam and Hua 1999a] in Tables 2 and 3. Afterwards, as shown in Figure 2, the correctness of using the size effect, is assessed by comparing the results from an isotropic homogeneous conical nanoshell with that of [Zeighampour and Tadi Beni 2014a] based on the modified couple stress theory. Besides, according to Table 2, the size effect which leads to increase in natural frequency are shown correctly. By way of comparison, the dimensionless natural frequency is calculated based on $\Omega=\omega R \sqrt{\rho\left(1-v^{2}\right) / E}$. 


\begin{tabular}{|lcccc|}
\hline$n$ & [Irie et al. 1984] & [Jin et al. 2014] & Present & Present $l=h$ \\
\hline 1 & 0.5462 & 0.5462 & 0.5988 & 0.6791 \\
2 & 0.631 & 0.6309 & 0.6371 & 0.6594 \\
3 & 0.5065 & 0.5063 & 0.5101 & 0.5511 \\
4 & 0.3947 & 0.3943 & 0.4044 & 0.4852 \\
5 & 0.3348 & 0.3340 & 0.3436 & 0.4975 \\
6 & 0.3248 & 0.3239 & 0.3290 & 0.5748 \\
7 & 0.3524 & 0.3213 & 0.3264 & 0.6833 \\
8 & 0.4033 & 0.4022 & 0.3934 & 0.8386 \\
9 & 0.4684 & 0.4673 & 0.4635 & 1.0227 \\
\hline
\end{tabular}

Table 2. Comparison of frequency parameter $\Omega$ for the conical shell. $(E=211 \mathrm{GPa}$, $\left.v=0.3, \rho=7800 \mathrm{~kg} \mathrm{~m}^{-3}, R_{2}=1 \mathrm{~m}, h / R_{2}=0.01, \alpha=45, L \sin (\alpha) / R_{2}=0.5\right)$.

\begin{tabular}{|cccccccccc|}
\hline \multicolumn{4}{c}{$\alpha=30^{\circ}$} & \multicolumn{3}{c}{$\alpha=45^{\circ}$} & \multicolumn{3}{c|}{$\alpha=60^{\circ}$} \\
$n$ & $\mathrm{~A}^{\dagger}$ & $\mathrm{B}^{\dagger \dagger}$ & Present & $\mathrm{A}^{\dagger}$ & $\mathrm{B}^{\dagger \dagger}$ & Present & $\mathrm{A}^{\dagger}$ & $\mathrm{B}^{\dagger \dagger}$ & Present \\
\hline 2 & 0.7910 & 0.8420 & 0.8297 & 0.6879 & 0.7655 & 0.7534 & 0.5722 & 0.6348 & 0.6301 \\
3 & 0.7284 & 0.7376 & 0.7329 & 0.6973 & 0.7212 & 0.7135 & 0.6001 & 0.6238 & 0.6208 \\
4 & 0.6352 & 0.6362 & 0.6365 & 0.6664 & 0.6739 & 0.6699 & 0.6054 & 0.6145 & 0.6130 \\
5 & 0.5531 & 0.5528 & 0.5552 & 0.6304 & 0.6323 & 0.6309 & 0.6077 & 0.6111 & 0.6102 \\
6 & 0.4949 & 0.4950 & 0.4972 & 0.6032 & 0.6035 & 0.6029 & 0.6159 & 0.6171 & 0.6158 \\
7 & 0.4653 & 0.4661 & 0.4662 & 0.5918 & 0.5921 & 0.5905 & 0.6343 & 0.6350 & 0.6322 \\
8 & 0.4654 & 0.4660 & 0.4629 & 0.5992 & 0.6001 & 0.5962 & 0.6650 & 0.6660 & 0.6607 \\
9 & 0.4892 & 0.4916 & 0.4848 & 0.6257 & 0.6273 & 0.6202 & 0.7084 & 0.7101 & 0.7016 \\
\hline
\end{tabular}

Table 3. Comparison of frequency parameter $\Omega$ for the conical shell. ( $E=211 \mathrm{GPa}$, $\left.v=0.3, \rho=7800 \mathrm{kgm}^{-3}, R_{2}=1 \mathrm{~m}, h / R_{2}=0.01, L \sin (\alpha) / R_{2}=0.25\right)$. $\dagger$ Values in column A are from [Irie et al. 1984]. ††Values in B are from [Lam and Hua 1999a].

As is visible, according to [Hua 2000; Lam and Hua 1997; 1999b; Sofiyev et al. 2009] using the Galerkin method, the comparison study is carried out for $n \geq 2$ in order to establish convergent validity; therefore, in this study according to Table 2, the obtained results have good consistency for $n \geq 2$; besides, in order to gain confidence on the accuracy of the current study, an additional comparison is carried out in different apex angle according to Table 3. As is clear, the results have appropriate precision, according to Table 2, Table 3 and Figure 2.

4.2. Effects of size parameter and apex angle on natural frequency. Figure 3 illustrates the effect of dimensionless length scale parameter on natural frequency for $\alpha=30^{\circ}$ according to the modified couple stress theory. As can be seen in Figure 3, decrease in the dimensionless length scale parameter is accompanied by increase in natural frequency according to the modified couple stress theory, and this increase is intensified with the increase in the gradient index $N$. In fact, decrease in $h / l$ stiffens the conical shell, leading to increase in natural frequency. Also, as $N=0$ is according to the aluminum shell 


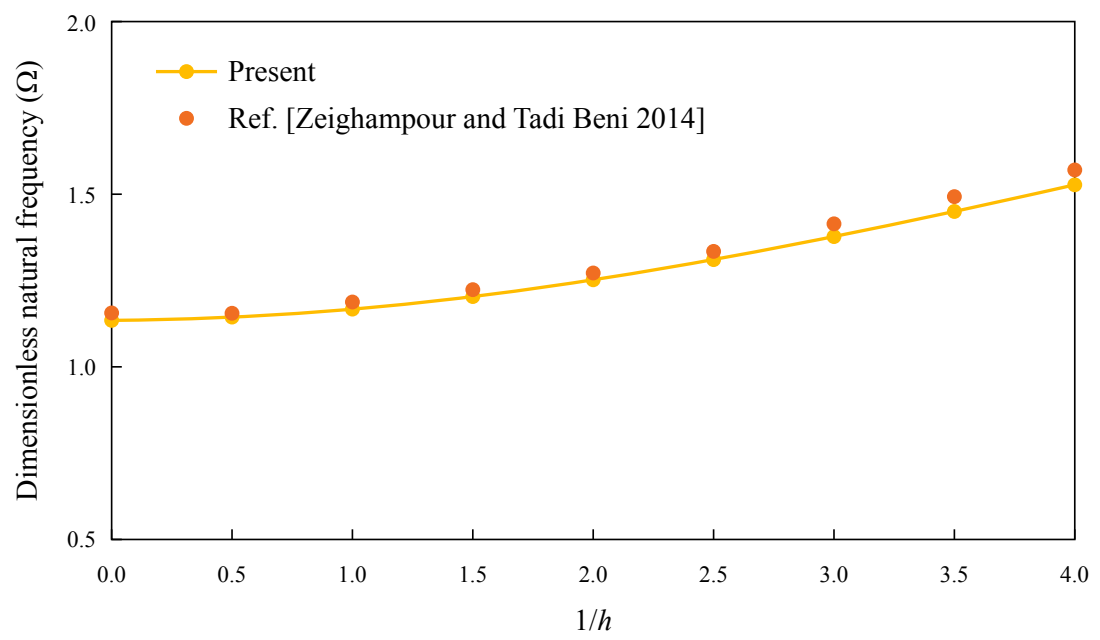

Figure 2. Comparison of dimensionless natural frequency versus dimensionless length scale parameter for SWCNC.

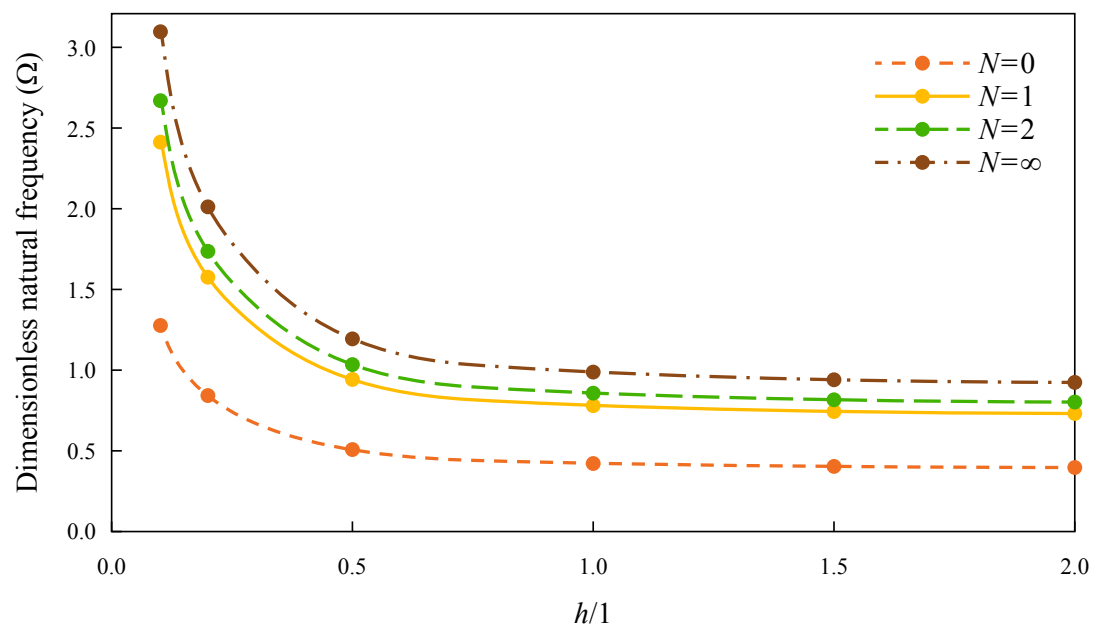

Figure 3. Effects of dimensionless length scale parameter on natural frequency based on modified couple stress theory $\alpha=30^{\circ}$.

and $N=$ infinite is according to the ceramic shell, an increase in the gradient index is accompanied by an increase in Young's modulus of FG conical nanoshell, which stiffens the shell and results in increasing the natural frequency.

Figure 4 displays the effect of dimensionless length scale parameter on natural frequency for $\alpha=60^{\circ}$ based on the modified couple stress theory. As can be seen in Figures 3 and 4, as the apex angle increases, the natural frequency increases with the decrease in dimensionless length scale parameter, and the effect of increase in the gradient index on the natural frequency can still be seen; however, with the increases of the apex angle, the effects of these two parameters, length scale parameter and gradient index, on the increase of natural frequency are weakened. 


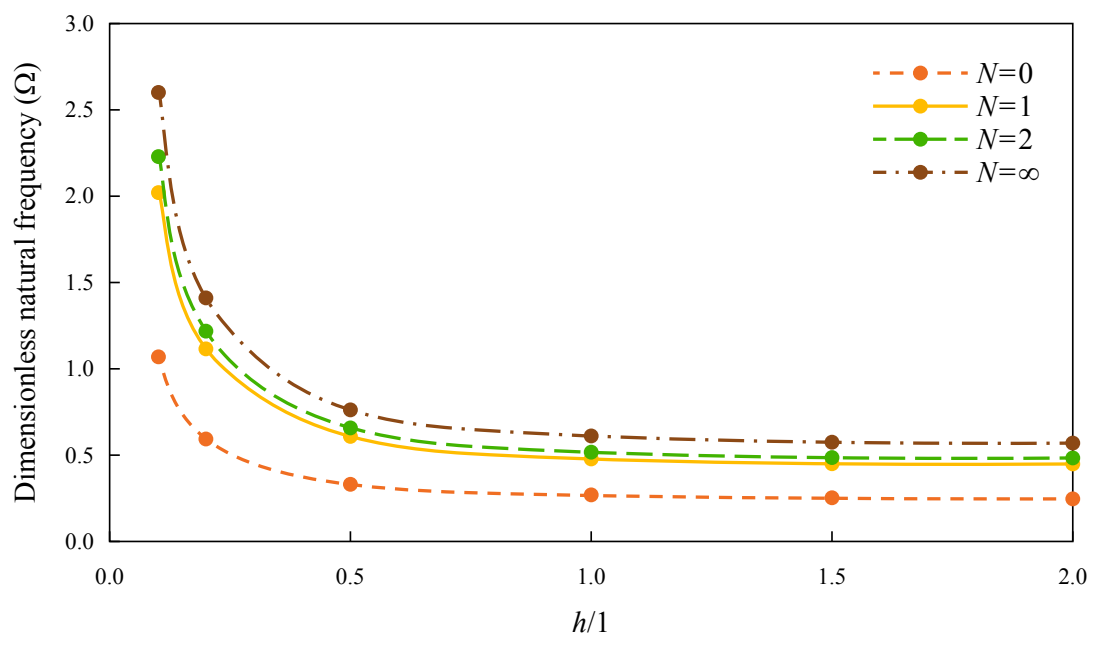

Figure 4. Effects of dimensionless length scale parameter on natural frequency based on modified couple stress theory $\alpha=60^{\circ}$.

4.3. Effects of length besides circumferential and axial wave number on natural frequency. Figures 5, 6 show the effect of length parameter; in addition, the circumferential and axial wave numbers on the natural frequency. As can be seen, an increase in the circumferential and axial wave numbers leads to increase in the natural frequency. On the other hand, the decrease in length parameter induced the decrease in stability in the shell leading to increase in natural frequency intensifies the increasing effect of circumferential and axial wave numbers on the increase in natural frequency. Therefore, in $l=h$, as length parameter decreases from $L=2 R_{1}$ to $L=R_{1}$, and the circumferential wave number increases from $n=1$ to $n=5$, the natural frequency increases from 1.69 to 2.3 for $L=2 R_{1}$ and from 3.9 to 9 for $L=R_{1}$. In addition, according to the illustration, increase in the length scale parameter has an increasing impact on the effect of length parameter and circumferential and axial wave numbers on natural frequency. Thus, in $L=R_{1}$, as the axial wave number changes from $m=1$ to $m=5$, the dimensionless natural frequency increases from 1.95 to 13.6 in $l=h$ and in $l=2 h$, the natural frequency increases from 2.77 to 22.1. Also, in $n=2$, the decrease in length parameter from $L=2 R_{1}$ to $L=R_{1}$ leads to an increase in natural frequency from 1.36 to 3.7 in $l=h$ and from 1.77 to 5.66 in $l=2 h$.

\section{Conclusion}

Using the modified couple stress theory, a new formulation for the FG truncated conical thin shell is developed in this paper. The size effect is considered using the modified couple stress theory, and material distribution in the FG conical shell is assumed according to the power law distribution as continuously variable along shell thickness. Governing equations as well as classical and nonclassical boundary conditions are derived using Hamilton's principle, and, in the special case, the free vibration of the simply supported FG conical nanoshell is investigated. Also, the effects of parameters such as length scale parameter, apex angle, length, and circumferential and axial wave numbers on natural frequency is studied. Increase in natural frequency induced by increase in length scale parameter is shown based on the modified couple stress theory and compared with the results of classical continuum theory. Moreover, 


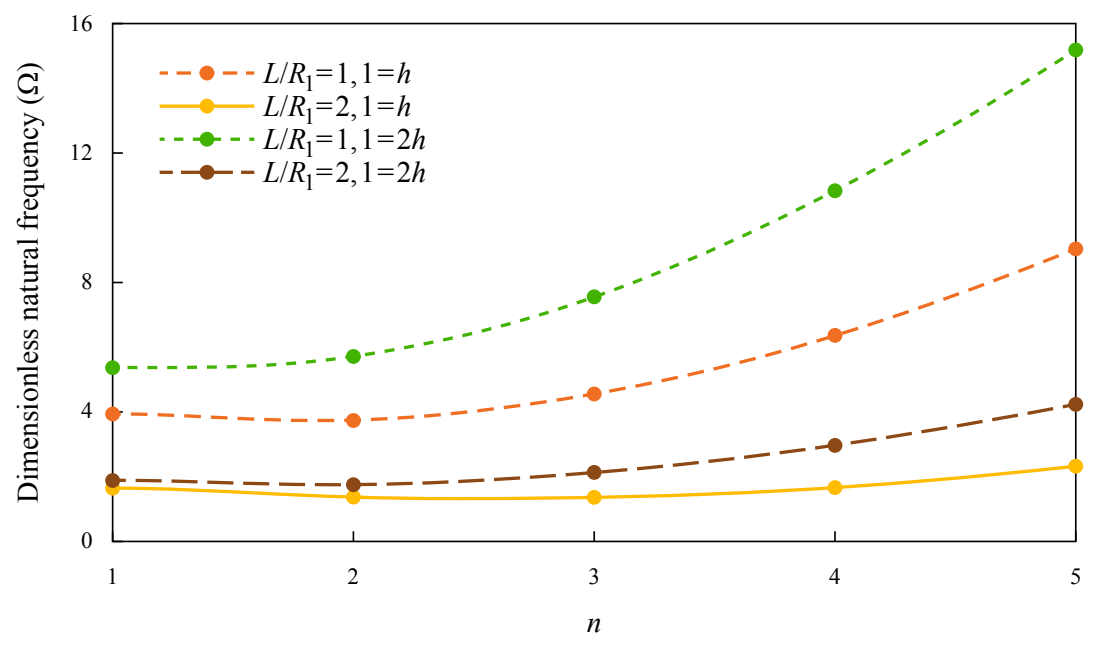

Figure 5. Effects of circumferential wave number and dimensionless length on dimensionless natural frequency, $\alpha=30^{\circ}, N=1$.

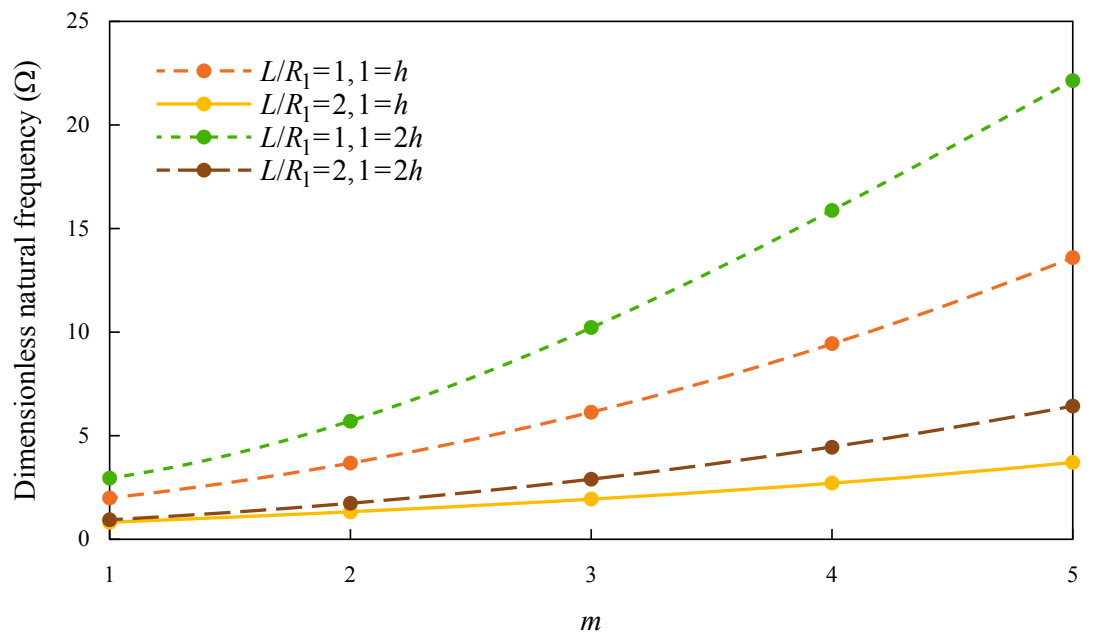

Figure 6. Effects of axial wave number and dimensionless length on dimensionless natural frequency, $\alpha=30^{\circ}, N=1$.

the increasing effect of length scale parameter on the natural frequency induced by the decrease in length and increase in circumferential and axial wave numbers is investigated as well.

\section{References}

[Abadyan et al. 2011] M. R. Abadyan, Y. Tadi Beni, and A. Noghrehabadi, "Investigation of elastic boundary condition on the pull-in instability of beam-type NEMS under van der Waals attraction", Proc. Eng. 10 (2011), 1724-1729.

[Abbasnejad et al. 2013] B. Abbasnejad, G. Rezazadeh, and R. Shabani, "Stability analysis of a capacitive fgm micro-beam using modified couple stress theory”, Acta Mech. Solida Sin. 26:4 (2013), 427-440. 
[Ansari et al. 2010] R. Ansari, M. Hemmatnezhad, and H. Ramezannezhad, "Application of HPM to the nonlinear vibrations of multiwalled carbon nanotubes", Numer. Methods Partial Differ. Equations 26:2 (2010), 490-500.

[Ansari et al. 2013] R. Ansari, R. Gholami, M. F. Shojaei, V. Mohammadi, and S. Sahmani, "Size-dependent bending, buckling and free vibration of functionally graded Timoshenko microbeams based on the most general strain gradient theory", Compos. Struct. 100 (2013), 385-397.

[Ansari et al. 2014] R. Ansari, M. F. Shojaei, V. Mohammadi, R. Gholami, and M. A. Darabi, "Nonlinear vibrations of functionally graded Mindlin microplates based on the modified couple stress theory", Compos. Struct. 114 (2014), 124-134.

[Anthoine 2000] A. Anthoine, "Effect of couple-stresses on the elastic bending of beams", Int. J. Solids Struct. 37:7 (2000), 1003-1018.

[Aydogdu and Taskin 2007] M. Aydogdu and V. Taskin, "Free vibration analysis of functionally graded beams with simply supported edges", Mater. Des. 28:5 (2007), 1651-1656.

[Barber 2011] J. R. Barber, Intermediate mechanics of materials, 2nd ed., Solid Mechanics and Its Applications 175, Springer, New York, 2011.

[Craciunescu and Wuttig 2003] C. M. Craciunescu and M. Wuttig, "New ferromagnetic and functionally graded shape memory alloys", ChemInform 34:39 (2003).

[Şimşek 2010] M. Şimşek, "Dynamic analysis of an embedded microbeam carrying a moving microparticle based on the modified couple stress theory”, Int. J. Eng. Sci. 48:12 (2010), 1721-1732.

[Şimşek and Reddy 2013] M. Şimşek and J. N. Reddy, "Bending and vibration of functionally graded microbeams using a new higher order beam theory and the modified couple stress theory", Int. J. Eng. Sci. 64 (2013), 37-53.

[Şimşek et al. 2013] M. Şimşek, T. Kocatürk, and Ş. D. Akbaş, "Static bending of a functionally graded microscale Timoshenko beam based on the modified couple stress theory", Compos. Struct. 95 (2013), 740-747.

[Dung et al. 2013] D. V. Dung, L. K. Hoa, N. T. Nga, and L. T. N. Anh, "Instability of eccentrically stiffened functionally graded truncated conical shells under mechanical loads”, Compos. Struct. 106 (2013), 104-113.

[Dung et al. 2014] D. V. Dung, L. K. Hoa, and N. T. Nga, "On the stability of functionally graded truncated conical shells reinforced by functionally graded stiffeners and surrounded by an elastic medium", Compos. Struct. 108 (2014), 77-90.

[Eringen 1980] A. C. Eringen, Mechanics of continua, 2nd ed., R. E. Krieger, Huntington, New York, 1980.

[Fu et al. 2004] Y. Fu, H. Du, W. Huang, S. Zhang, and M. Hu, "TiNi-based thin films in MEMS applications: A review”, Sens. Actuators A Phys. 112:2-3 (2004), 395-408.

[Furlotti et al. 2014] E. Furlotti, G. Giuliese, A. Pirondi, A. Zucchelli, and R. Palazzetti, "Numerical assessment of impact behavior of composite laminates with variable interply delamination strength", J. Proc. Mater. Sci. 3 (2014), 1467-1472.

[Hua 2000] L. Hua, "Frequency analysis of rotating truncated circular orthotropic conical shells with different boundary conditions", Compos. Sci. Technol. 60:16 (2000), 2945-2955.

[Irie et al. 1984] T. Irie, G. Yamada, and K. Tanaka, "Natural frequencies of truncated conical shells", J. Sound Vib. 92:3 (1984), $447-453$.

[Jin et al. 2014] G. Jin, X. Ma, S. Shi, T. Ye, and Z. Liu, "A modified Fourier series solution for vibration analysis of truncated conical shells with general boundary conditions", Appl. Acoust. 85 (2014), 82-96.

[Jomehzadeh et al. 2009] E. Jomehzadeh, A. R. Saidi, and S. R. Atashipour, "An analytical approach for stress analysis of functionally graded annular sector plates”, Mater. Des. 30:9 (2009), 3679-3685.

[Kahrobaiyan et al. 2012] M. H. Kahrobaiyan, M. Rahaeifard, S. A. Tajalli, and M. T. Ahmadian, "A strain gradient functionally graded Euler-Bernoulli beam formulation", Int. J. Eng. Sci. 52 (2012), 65-76.

[Kant et al. 2010] T. Kant, S. M. Shiyekar, and C. V. Subbaiah, "Higher order theories of functionally graded beams and plates", pp. 65-74 in IUTAM symposium on multi-functional material structures and systems, edited by B. Dattaguru et al., IUTAM Bookseries 19, Springer, Netherlands, 2010.

[Ke et al. 2012] L.-L. Ke, Y.-S. Wang, J. Yang, and S. Kitipornchai, "Nonlinear free vibration of size-dependent functionally graded microbeams", Int. J. Eng. Sci. 50:1 (2012), 256-267.

[Khalili et al. 2010] S. M. R. Khalili, A. A. Jafari, and S. A. Eftekhari, "A mixed Ritz-DQ method for forced vibration of functionally graded beams carrying moving loads", Compos. Struct. 92:10 (2010), 2497-2511. 
[Koiter 1964a] W. T. Koiter, "Couple stresses in the theory of elasticity, I", Proceedings of the Koninklijke Nederlandse Akademie van Wetenschappen, Series B 67 (1964), 17-29.

[Koiter 1964b] W. T. Koiter, "Couple stresses in the theory of elasticity, II", Proceedings of the Koninklijke Nederlandse Akademie van Wetenschappen, Series B 67 (1964), 30-44.

[Lam and Hua 1997] K. Y. Lam and L. Hua, "Vibration analysis of a rotating truncated circular conical shell", Int. J. Solids Struct. 34:17 (1997), 2183-2197.

[Lam and Hua 1999a] K. Y. Lam and L. Hua, "Influence of boundary conditions on the frequency characteristics of a rotating truncated circular conical shell”, J. Sound Vib. 223:2 (1999), 171-195.

[Lam and Hua 1999b] K. Y. Lam and L. Hua, "On free vibration of a rotating truncated circular orthotropic conical shell", Compos. B Eng. 30:2 (1999), 135-144.

[Leissa 1993] A. W. Leissa, Vibration of shells, American Inst. of Physics, Woodbury, NY, 1993.

[Li 2008] X.-F. Li, "A unified approach for analyzing static and dynamic behaviors of functionally graded Timoshenko and Euler-Bernoulli beams", J. Sound Vib. 318:4-5 (2008), 1210-1229.

[Li et al. 2014] D. H. Li, Y. Liu, and X. Zhang, "Low-velocity impact responses of the stiffened composite laminated plates based on the progressive failure model and the layerwise/solid-elements method", Compos. Struct. 110 (2014), 249-275.

[Malekzadeh and Daraie 2014] P. Malekzadeh and M. Daraie, "Dynamic analysis of functionally graded truncated conical shells subjected to asymmetric moving loads", Thin-Walled Struct. 84 (2014), 1-13.

[McFarland and Colton 2005] A. W. McFarland and J. S. Colton, "Role of material microstructure in plate stiffness with relevance to microcantilever sensors", J. Micromech. Microeng. 15:5 (2005), 1060.

[Mindlin 1964] R. D. Mindlin, "Micro-structure in linear elasticity", Arch. Ration. Mech. Anal. 16:1 (1964), 51-78.

[Mindlin and Tiersten 1962] R. D. Mindlin and H. F. Tiersten, "Effects of couple-stresses in linear elasticity", Arch. Ration. Mech. Anal. 11:1 (1962), 415-448.

[Mohammad Abadi and Daneshmehr 2014] M. Mohammad Abadi and A. R. Daneshmehr, "An investigation of modified couple stress theory in buckling analysis of micro composite laminated Euler-Bernoulli and Timoshenko beams", Int. J. Eng. Sci. 75 (2014), 40-53.

[Reddy 2000] J. N. Reddy, “Analysis of functionally graded plates”, Int. J. Numer. Methods Eng. 47:1-3 (2000), 663-684.

[Roque et al. 2013] C. M. C. Roque, D. S. Fidalgo, A. J. M. Ferreira, and J. N. Reddy, "A study of a microstructure-dependent composite laminated Timoshenko beam using a modified couple stress theory and a meshless method", Compos. Struct. 96 (2013), 532-537.

[Sahmani and Ansari 2013] S. Sahmani and R. Ansari, "On the free vibration response of functionally graded higher-order shear deformable microplates based on the strain gradient elasticity theory”, Compos. Struct. 95 (2013), 430-442.

[Sahmani et al. 2013] S. Sahmani, R. Ansari, R. Gholami, and A. Darvizeh, "Dynamic stability analysis of functionally graded higher-order shear deformable microshells based on the modified couple stress elasticity theory", Compos. B Eng. 51 (2013), 44-53.

[Sahoo and Singh 2014] R. Sahoo and B. N. Singh, "A new trigonometric zigzag theory for buckling and free vibration analysis of laminated composite and sandwich plates", Compos. Struct. 117 (2014), 316-332.

[Sankar 2001] B. V. Sankar, "An elasticity solution for functionally graded beams", Compos. Sci. Technol. 61:5 (2001), 689696.

[Shahba and Rajasekaran 2012] A. Shahba and S. Rajasekaran, "Free vibration and stability of tapered Euler-Bernoulli beams made of axially functionally graded materials", Appl. Math. Model. 36:7 (2012), 3094-3111.

[Sofiyev 2009] A. H. Sofiyev, "The vibration and stability behavior of freely supported FGM conical shells subjected to external pressure", Compos. Struct. 89:3 (2009), 356-366.

[Sofiyev et al. 2009] A. H. Sofiyev, M. H. Omurtag, and E. Schnack, "The vibration and stability of orthotropic conical shells with non-homogeneous material properties under a hydrostatic pressure", J. Sound Vib. 319:3-5 (2009), 963-983.

[Su et al. 2014] Z. Su, G. Jin, S. Shi, T. Ye, and X. Jia, "A unified solution for vibration analysis of functionally graded cylindrical, conical shells and annular plates with general boundary conditions”, Int. J. Mech. Sci. 80 (2014), 62-80. 
[Tadi Beni 2012] Y. Tadi Beni, "Use of augmented continuum theory for modeling the size dependent material behavior of nano-actuators", Iran. J. Sci. Technol. B 36:M1 (2012), 41-52.

[Tadi Beni and Abadyan 2013] Y. Tadi Beni and M. Abadyan, "Use of strain gradient theory for modeling the size-dependent pull-in of rotational nano-mirror in the presence of molecular force", Int. J. Mod. Phys. B 27:18 (2013), Art ID 1350083.

[Tadi Beni et al. 2012] Y. Tadi Beni, A. Koochi, A. S. Kazemi, and M. Abadyan, "Modeling the influence of surface effect and molecular force on pull-in voltage of rotational nano-micro mirror using 2-DOF model", Can. J. Phys. 90:10 (2012), 963-974.

[Tadi Beni et al. 2014] Y. Tadi Beni, A. Koochi, and M. Abadyan, "Using modified couple stress theory for modeling the size-dependent pull-in instability of torsional nano-mirror under Casimir force", Int. J. Optomechatronics 8:1 (2014), 47-71.

[Tadi Beni et al. 2015a] Y. Tadi Beni, F. Mehralian, and H. Razavi, "Free vibration analysis of size-dependent shear deformable functionally graded cylindrical shell on the basis of modified couple stress theory", Compos. Struct. 120 (2015), 65-78.

[Tadi Beni et al. 2015b] Y. Tadi Beni, F. Mehralian, and H. Zeighampour, "The modified couple stress functionally graded cylindrical thin shell formulation", Mech. Adv. Mater. Struct. (online publication April 2015).

[Tajalli et al. 2013] S. A. Tajalli, M. Rahaeifard, M. H. Kahrobaiyan, M. R. Movahhedy, J. Akbari, and M. T. Ahmadian, "Mechanical behavior analysis of size-dependent micro-scaled functionally graded Timoshenko beams by strain gradient elasticity theory", Compos. Struct. 102 (2013), 72-80.

[Tornabene and Viola 2013] F. Tornabene and E. Viola, "Static analysis of functionally graded doubly-curved shells and panels of revolution", Meccanica (Milano) 48:4 (2013), 901-930.

[Tornabene et al. 2015] F. Tornabene, N. Fantuzzi, E. Viola, and R. C. Batra, "Stress and strain recovery for functionally graded free-form and doubly-curved sandwich shells using higher-order equivalent single layer theory", Compos. Struct. 119 (2015), $67-89$.

[Toupin 1962] R. A. Toupin, "Elastic materials with couple-stresses", Arch. Ration. Mech. Anal. 11:1 (1962), 385-414.

[Viola et al. 2012] E. Viola, L. Rossetti, and N. Fantuzzi, "Numerical investigation of functionally graded cylindrical shells and panels using the generalized unconstrained third order theory coupled with the stress recovery", Compos. Struct. 94:12 (2012), 3736-3758.

[Viola et al. 2014] E. Viola, L. Rossetti, N. Fantuzzi, and F. Tornabene, "Static analysis of functionally graded conical shells and panels using the generalized unconstrained third order theory coupled with the stress recovery", Compos. Struct. 112 (2014), 44-65.

[Wang et al. 2013] L. Wang, Y. Y. Xu, and Q. Ni, "Size-dependent vibration analysis of three-dimensional cylindrical microbeams based on modified couple stress theory: A unified treatment", Int. J. Eng. Sci. 68 (2013), 1-10.

[Witvrouw and Mehta 2005] A. Witvrouw and A. Mehta, "The use of functionally graded poly-SiGe layers for MEMS applications", Mater. Sci. Forum 492-493 (2005), 255-260.

[Xie et al. 2014] X. Xie, G. Jin, Y. Yan, S. X. Shi, and Z. Liu, "Free vibration analysis of composite laminated cylindrical shells using the Haar wavelet method", Compos. Struct. 109 (2014), 169-177.

[Yang et al. 2002] F. Yang, A. C. M. Chong, D. C. C. Lam, and P. Tong, "Couple stress based strain gradient theory for elasticity", Int. J. Solids Struct. 39:10 (2002), 2731-2743.

[Ying et al. 2008] J. Ying, C. F. Lü, and W. Q. Chen, "Two-dimensional elasticity solutions for functionally graded beams resting on elastic foundations", Compos. Struct. 84:3 (2008), 209-219.

[Yoon et al. 2005] J. Yoon, C. Q. Ru, and A. Mioduchowski, "Vibration and instability of carbon nanotubes conveying fluid", Compos. Sci. Technol. 65:9 (2005), 1326-1336.

[Zeighampour and Tadi Beni 2014a] H. Zeighampour and Y. Tadi Beni, "Analysis of conical shells in the framework of coupled stresses theory", Int. J. Eng. Sci. 81 (2014), 107-122.

[Zeighampour and Tadi Beni 2014b] H. Zeighampour and Y. Tadi Beni, "Size-dependent vibration of fluid-conveying doublewalled carbon nanotubes using couple stress shell theory", Physica E 61 (2014), 28-39.

[Zeighampour et al. 2015] H. Zeighampour, Y. Tadi Beni, and F. Mehralian, "A shear deformable conical shell formulation in the framework of couple stress theory", Acta Mech. 226:8 (2015), 2607-2629.

[Zeverdejani and Tadi Beni 2013] M. K. Zeverdejani and Y. Tadi Beni, "The nano scale vibration of protein microtubules based on modified strain gradient theory”, Curr. Appl. Phys. 13:8 (2013), 1566-1576. 
[Zhang et al. 2005] Y. Zhang, G. Liu, and X. Han, "Transverse vibrations of double-walled carbon nanotubes under compressive axial load", Phys. Lett. A 340:1-4 (2005), 258-266.

[Zhao and Liew 2011] X. Zhao and K. M. Liew, "Free vibration analysis of functionally graded conical shell panels by a meshless method", Compos. Struct. 93:2 (2011), 649-664.

[Zhao and Pedroso 2008] J. Zhao and D. Pedroso, "Strain gradient theory in orthogonal curvilinear coordinates", Int. J. Solids Struct. 45:11-12 (2008), 3507-3520.

Received 6 Jan 2015. Revised 2 Oct 2015. Accepted 16 Dec 2015.

YAGHOUB TADI BENI: tadi@eng.sku.ac.ir

Mechanical Engineering, Shahrekord University, Rahbar Boulevard, Shahrekord 88581, Iran

FAHIMEH MEHRALIAN: fahimeh.mehralian@yahoo.com

Mechanical Engineering, Shahrekord University, Rahbar Boulevard, Shahrekord 88581, Iran 


\title{
CONDITIONS FOR THE LOCALISATION OF PLASTIC DEFORMATION IN TEMPERATURE SENSITIVE VISCOPLASTIC MATERIALS
}

\author{
Martin K. Paesold, Andrew P. Bassom, Klaus Regenauer-Lieb and Manolis Veveakis
}

\begin{abstract}
We study the onset of localisation of plastic deformation for a class of materials that exhibit both temperature and rate sensitivity. The onset of localisation is determined via an energy bifurcation criterion, defined by the postulate that viscoplastic materials admit a critical (mechanical) energy input above which deformation becomes unstable and plastic localisation ensues. In analogy to the classical concepts of mechanics, the conditions for the onset of localisation in temperature-sensitive viscoplastic materials are reached at a critical stress. However, it is shown that in viscoplastic materials a material bifurcation occurs when the heat supply through mechanical work surpasses the diffusion capabilities of the material. This transition from near-isothermal stable evolution to near-adiabatic thermal runaway is the wellknown concept of shear heating. Here, it is generalised and the correspondence between this runaway instability and the localisation of plastic deformation in solid mechanics is detailed. The obtained phase space controlling the localisation is shown to govern the evolution of the system in the postyield regime. These results suggest that the energy balance essentially drives the evolution of the plastic deformation and therefore constitutes a physics-based hardening law for thermoviscoplastic materials.
\end{abstract}

\section{Introduction}

In this work, we present the hypothesis that the classical criterion for localisation can be generalised from their isothermal and adiabatic limits through an extension of an energy bifurcation criterion suggested in earlier studies [Cherukuri and Shawki 1995a; 1995b]. The criterion involves an analytical analysis of the multiple steady states followed by a numerical analysis of the transients. We showcase the development of the criterion, highlight its relation to the characteristics of hyperbolic differential equations (slip line fields) and present a first numerical example. The problem of the localisation of plastic deformation is commonly considered solved once both the spatial configuration of localisation and the necessary loading conditions for its onset are extracted. The classical theory [Hill 1950; 1962; Rudnicki and Rice 1975; Rice 1977] of rate and temperature independent materials, provides a platform upon which modern solid mechanics is built. These works suggest that the spatial configuration as well as the critical loading conditions for the onset of localisation can be obtained from the stationary limit of a material dependent wave equation.

In particular, the slip line field theory for ideal rigid plastic materials has been developed in the middle of the last century [Hill 1950] and successfully applied to metal forming processes. The theory is based on solving the hyperbolic differential equations of mass and momentum balance (at its stationary limit)

This work was supported by iVEC through the use of advanced computing resources located at the iVEC@Murdoch facility and MKP is grateful for financial support through the Australian Postgraduate Award and an International Postgraduate Research Scholarship.

Keywords: bifurcation analysis, localisation of plastic deformation, energy balance, slip lines. 
and provides a closed form solution for the failure of such an idealised material using the method of characteristics. The slip line field theory [Hill 1962] also forms the background for the development of a criterion of localisation of plastic deformation stemming from a material bifurcation [Rudnicki and Rice 1975; Rice 1977]. The study of this material bifurcation was the subject of the early approaches of accelerating waves in rate and temperature independent solids [Hill 1962; Rudnicki and Rice 1975] and shows that localisation instabilities occur at the stationary wave limit of the linear elasto-plastic wave equation

$$
L_{i k} \eta_{k}=\rho c^{2} \eta_{i}
$$

In this eigenvalue problem $\eta_{i}$ is the vector of the jump of the velocity gradient, $c$ is the material velocity of the accelerating wave and $\rho$ the material density. The properties of the rate- and temperature-independent material are encapsulated in the acoustic tensor

$$
L_{i k}=v_{j} C_{i j k l}^{\mathrm{ep}} v_{l},
$$

where $C_{i j k l}^{\mathrm{ep}}$ is the elasto-plastic stiffness matrix of the material obeying an incremental elasto-plastic response $\dot{\sigma}_{i j}=C_{i j k l}^{\mathrm{ep}} \dot{\epsilon}_{k l}$. The vectors $v_{i}$ are the unit vectors of the discontinuity imposed by the propagating acceleration wave. According to the theory of localisation of plastic deformation [Hill 1962; Rudnicki and Rice 1975], localised failure occurs at the stationary limit of the propagating wave when $c=0$. In this regime, nontrivial solutions are obtained only when the determinant of the acoustic tensor is zero and this condition allows for the calculation of both the orientation of the localised plane given by the vectors $v_{i}$, and the critical stress ratio for the onset of localisation, given by the critical value of the tangent modulus.

Although slip line field theory has played an undeniable role in underpinning the theory of plasticity, and has still some use in the limit analysis and design [Khan et al. 2008], it has been superseded by advanced numerical techniques that are capable of modelling nonlinear, elastic, viscous and plastic materials [Needleman and Tvergaard 1992]. The main drawback of the slip line field theory was that it cannot be used for rate and temperature dependent constitutive laws which significantly hampered the applicability to modern engineering applications since the importance of temperature is well known in constitutive properties of most materials. Soil, rocks and ceramics are significantly influenced by temperature with strain localisation being strongly affected by thermal loading [Hückel and Baldi 1990; Hückel and Pellegrini 2002]. In polymers and polycarbonates temperature and strain rate are key parameters influencing the response of the material, even at ambient conditions [Bauwens-Crowet et al. 1974]. Finally, even in the analogue rigid materials for which the slip line field theory was developed, i.e., metals, temperature was shown to be important. This becomes obvious in particular under conditions of high speed deformation or at large strain where mechanical work is dissipated and the effects of heat becomes important. An excellent example is the thermal cross that is often observed during forging of mild steel caused by localised plastic dissipation on slip lines [Johnson et al. 1964].

The thermal cross becomes visible as heat lines owing to reaching temperatures of around $680{ }^{\circ} \mathrm{C}$ and their pattern [Johnson et al. 1964, Figures 3 and 4]) closely resembles the original slip lines calculated in theoretical plasticity [Hill 1950]. The coincidence of heat lines and slip lines suggests a strong relationship and calls for an extension of the original theory beyond isothermal conditions. Later studies [Benallal and Lemaitre 1991; 2004] generalised the localisation concepts and extended the acoustic tensor 
criterion to the realm of coupled thermomechanical response for rate-independent materials, involving an updated formulation of the acoustic tensor for the limits of isothermal and adiabatic conditions.

However, when dealing with rate-dependent thermoplastic coupling, the mathematical study of the eigenvalue problem (1-1) of plasticity breaks down. The procedure for determining the conditions for the onset of localised deformation differs significantly from that of rate-independent materials [Anand et al. 1987]. In this regime, the conditions for the loss of ellipticity that express the material instability leading to localisation is approached through analyses of the full system of field equations, rather than seeking for the loss of ellipticity of the momentum balance. These techniques were first introduced for one-dimensional plastic shear deformation of nonlinear viscous fluids [Gruntfest 1963; Clifton 1980; Bai 1981; 1982]. The one-dimensional problem of simple shear of a temperature dependent viscoplastic layer has also been treated semianalytically [Chen et al. 1989; Leroy and Molinari 1992]. These concepts gave rise to the proposition of an energy based localisation theory in which instabilities emerge when the mechanical input rate rises significantly leading to a departure from the near isothermal limit towards the near adiabatic limit [Cherukuri and Shawki 1995b; 1995a]. Since the energy equation can provide information about the time evolution of the system, this regime has been extensively studied in earth sciences [Regenauer-Lieb et al. 2013a; Regenauer-Lieb et al. 2013b] for one-dimensional failure patterns seen in landslides [Veveakis et al. 2007] and fault mechanics [Veveakis et al. 2010].

The adiabatic limit of the energy equation, also known as adiabatic shear bands, was the focus of considerable research efforts during past decades in material sciences [Gruntfest 1963]. It is of particular interest since the adiabatic shear limit can act as a precursor to failure, irrespective of its mode (ductile or brittle) [Dodd and Bai 2012]. In spite of the apparent relationship suggested by observations such as reported by Johnson et al. [Johnson et al. 1964], a generalised slip line field theory that extends Hill's theory to nonlinear thermoviscoplastic materials is not yet developed. In this study we show that such a theory can be obtained by expanding the conditions necessary for the loss of ellipticity of the momentum equations, in the realm of coupled thermomechanical problems for temperature-dependent, viscoplastic materials. This generalisation of the slip line field theory is two-pronged: firstly, at the limit of stationary thermomechanical wave propagation (following the classical concepts of mechanics) the stress equilibrium conditions define a spatial pattern of failure and dissipation (the product of stress and velocity gradient). Secondly, the energy balance supplies the necessary conditions for the loss of ellipticity that would lead to a jump in the dissipation. Further on, the transient analysis of the system provides the evolution of plastic deformation from near-isothermal to near-adiabatic conditions and verifies the results of the stationary analysis.

In the remainder of this article we present a detailed mathematical approach to the problem. In Section 2 the continuum thermomechanical framework considered in this article is described. Section 3 extends the concepts of the slip line field theory from ideal plastic to viscoplastic materials. This entails a generalisation of Hencky's and Geiringer's equations that determine the geometry and the onset of localisation, respectively. These results are compared to finite element simulations of simple geometries and Johnson's heat lines (see [Johnson et al. 1964, Figures 3 and 4]) in Section 4. We conclude with a discussion on the importance of the obtained results in Section 5. In the appendices more details on the mathematical derivations are given and in Appendix A the energy budget of a viscoplastic material is discussed, Appendix B details the derivation of the slip lines and Appendix $\mathrm{C}$ gives a perturbation analysis of the energy equation. 


\section{Fundamental principles of continuum thermomechanics}

Here, we restrict ourselves to general plane strain loading and neglect the effect of gravity and inertia terms so that the stress equilibrium reads

$$
\begin{aligned}
& \frac{\partial \sigma_{11}}{\partial x_{1}}+\frac{\partial \sigma_{12}}{\partial x_{2}}=0, \\
& \frac{\partial \sigma_{21}}{\partial x_{1}}+\frac{\partial \sigma_{22}}{\partial x_{2}}=0 .
\end{aligned}
$$

Further, we adopt the stress decomposition $\sigma_{i j}=p \delta_{i j}+s_{i j}$, with $p$ the volumetric mean stress and $s_{i j}$ the deviatoric stress. For such a problem, we formulate the governing equations in the equivalent coordinate system where the stress tensor is rotated such that its elements are the mean stress $p=I_{1} / 3$ and the von Mises stress $q=\sqrt{3 J_{2}}$. In these expressions $I_{1}=\operatorname{tr}\left(\sigma_{i j}\right)$ is the first invariant of the stress tensor and $J_{2}=s_{i j} s_{i j} / 2$. The corresponding coordinate transformation dates back to Levy [Hill 1950] and is given through the Mohr circle transformation

$$
\sigma_{11}=p-q \sin 2 \psi, \quad \sigma_{22}=p+q \sin 2 \psi, \quad \sigma_{12}=\sigma_{21}=q \cos 2 \psi,
$$

where $\psi$ is the rotation angle of the coordinate system.

2A. Energy considerations. In this study we neglect higher-order energy terms in the energy equation. These terms are expressed in terms of a second derivative of the Helmholtz free energy, $\frac{\partial^{2} \psi}{\partial \psi \partial T} \dot{\xi}$ and are of secondary importance when studying the quasistatic the problem of localisation of viscoplastic deformation. Frequently this is the case because $\dot{\xi}=0$ at steady state. Such a case is the thermoelastic heating term (see Appendix A) in which case the internal state variable is the elastic strain. Under this assumption, the energy balance combined with the second law of thermodynamics can be written as

$$
\rho c \frac{\partial T}{\partial t}=\alpha\left(\frac{\partial^{2}}{\partial x_{1}^{2}}+\frac{\partial^{2}}{\partial x_{2}^{2}}\right) T+\Phi-r_{\text {sink }},
$$

where the right hand side represents the temporal change of energy within a volume element due to heat flux and mechanical dissipation

$$
\Phi=\beta \sigma_{i j} \dot{\varepsilon}_{i j}^{p},
$$

and an energy sink $r_{\text {sink }}$ due to a postlocalisation transformation such as melting or endothermic chemical reactions [Rosakis et al. 2000]. The constants in (2-4)-(2-5) denote the material density $\rho$, the specific heat capacity $c$, heat conductivity $\alpha$ and the Taylor-Quinney coefficient $\beta$ [Taylor and Quinney 1934]. This coefficient quantifies the amount of mechanical work converted to heat and $\beta$ is of particular importance in the field of thermodynamics with internal state variables, as it incorporates the evolution of all the internal state variables $\xi$ of the system, as evident from its definition [Alevizos et al. 2014; Veveakis et al. 2014]

$$
\beta=1-\frac{Y \dot{\xi}}{\sigma_{i j} \dot{\varepsilon}_{i j}^{p}} .
$$

In this expression $Y$ is a thermodynamic potential, dual in energy with the internal state variable $\xi$. 
2B. Constitutive modelling. We first split the strain rate into elastic (reversible) and plastic (irreversible) parts $\dot{\varepsilon}_{i j}=\dot{\varepsilon}_{i j}^{e}+\dot{\varepsilon}_{i j}^{p}$. For the elastic component we adopt a linear elastic law of the form

$$
\dot{\varepsilon}_{i j}^{e}=C_{i j k l}^{-1} \dot{\sigma}_{k l} \text {. }
$$

For the irreversible part, we assume that the Helmholtz free energy is invertible, such that the evolution of the plastic strain depends on the stress and temperature through a smooth function of the plastic potential $g\left(\sigma_{i j}, T\right)$. We focus on temperature contributions that act independently of the stress such that the viscoplastic flow law can be decomposed as

$$
\dot{\varepsilon}_{i j}^{p}=\dot{\varepsilon}_{0} e^{-T_{0} / T}\langle f(g)\rangle \frac{\partial g\left(\sigma_{i j}\right)}{\partial \sigma_{i j}},
$$

where the activation temperature is denoted by $T_{0}$, and the Macaulay brackets $\langle\cdot\rangle$ ensure zero plastic strain before yield [Freed and Walker 1993]. This decomposition is supported by experimental data at elevated temperatures, below the phase transition temperature of the material [Bauwens-Crowet et al. 1974] and the two most representative constitutive responses of temperature and rate dependent materials are an Arrhenius-type dependency on temperature, with either a power-law or an exponential dependency on stress. The function $f(g)$ is an arbitrary scalar flow stress function. For the example of $J_{2}$ associative viscoplasticity the viscoplastic flow law takes the form

$$
\dot{\varepsilon}_{i j}^{p}=\dot{e}_{i j}^{p}=\dot{\varepsilon}_{0} e^{-T_{0} / T}\langle f(g)\rangle \frac{\partial g}{\partial s_{i j}}=\dot{\varepsilon}_{0}\left\langle\frac{\sqrt{q}}{k}-1\right\rangle \frac{s_{i j}}{q} e^{-T_{0} / T},
$$

where $\dot{e}_{i j}^{p}$ is the deviatoric part of the strain-rate plastic and $k$ is the yield stress [Perzyna 1966]. The flow stress function is in principle dependent on temperature, plastic strain and any other internal state variable, but in this study we will not consider these dependencies for simplicity in the mathematical treatment.

The exact form of the constitutive equation is not prescribed during the analysis of the bifurcation in order to emphasise the generic nature of the formulation, where the onset of plastic deformation is derived from the basic assumptions of the energetics. The only important aspect of the constitutive response of the material is that it must obey a viscoplastic relationship linking the plastic strain-rate (i.e., plastic strain increment) with the stress, in contrast to rate independent plasticity where an incremental relationship between plastic strain increment and stress increment is required. The viscoplastic law is required so that in the steady-state limit of the equations $\left(\dot{\sigma}_{i j}=\dot{T}=0\right)$ the mechanical dissipation remains nonzero.

The formulation therefore encompasses most classes of physical behaviour described in the summary of constitutive laws for viscoplastic materials [Chaboche 2008]. Since the rate-independent plasticity case can be deduced from the equations of viscoplasticity as a limiting case [Chaboche 1977; Lubliner 2008], the presented formulation can be seen as a generic framework for temperature-sensitive plasticity. For a more detailed discussion on the constitutive concepts of viscoplasticity the reader is encouraged to consult the review article by Chaboche [2008].

As shown in earlier studies [Leroy and Molinari 1992; Cherukuri and Shawki 1995b; Veveakis et al. 2010], the choice of the form of the temperature dependence of the plastic flow law is not central for the results of the present study. Those studies have shown that any nonlinear temperature dependence 
leads to the same physical behaviour with the Arrhenius type exponential dependency being the one that allows for the most convenient mathematical treatment.

\section{Steady state analysis: generalisation of the slip line field theory}

We start by studying the steady-state limit of the system, in which $\dot{T}=\dot{\sigma}_{i j}=0$. Therefore, in this regime the elastic contribution (2-7) is neglected and the problem reduces to that of the study of the response of a rigid (visco-)plastic material. This setting can therefore be considered to be a direct extension of the slip line field theory to thermoviscoplastic materials. We note that in the present formulation the temperature equation (2-4) is active only when dissipation is nonzero. Since this is achieved in the postyield regime, we expect that the orientation of possible localisation planes would arise from the characteristics of the stress equilibrium, in accordance with the theory of plasticity [Hill 1950].

We therefore anticipate that given an arbitrary set of slip lines, two distinctly different cases of material response can be identified from the energy balance equation. The plastic material may deform homogeneously across the whole domain, and therefore across the slip lines; alternatively the material deforms in a localised manner along the slip lines (the equivalent velocity gradient discontinuities in the classical case). In our formulation the transition becomes an energy based transition expressed through the multiple steady states which are possible. The complete field approach of the present work consists of the identification of the generalised patterns of slip lines given by an arbitrary yield surface and the derivation of the conditions for localised plastic deformation along these slip lines.

3A. Generalised Hencky's equations. At the point of initial yield, where the temperature equation is inactive, the response of the system is governed by the stress equilibrium equations. We assume a generalised yield surface at a reference temperature of the form $q=q_{Y}(p)$. In order to find the characteristics of the hyperbolic differential stress equilibrium equations (slip lines) we substitute the Levy stress transformations, (2-3), into the stress equilibrium equations, (2-1) and (2-2). The mathematical treatment is detailed in Appendix B (see also [Lubliner 2008, Chapter 5]) and the results are summarised below.

The slip lines are parametrised in terms of the arc-length $s$ and the slopes of the characteristics along the $x_{1}$ - and $x_{2}$-axes read

$$
\frac{\partial x_{1}}{\partial s_{k}}=\frac{\mu^{(k)}}{\sqrt{1+\mu^{(k) 2}}}, \quad \frac{\partial x_{2}}{\partial s_{k}}=\frac{1}{\sqrt{1+\mu^{(k) 2}}} \quad(k=1,2),
$$

where $\mu^{(k)}$ is the $k$-th left eigenvalue of the stress equilibrium equations (see Appendix B). For a general yield surface $q(p)$

$$
\mu^{(1,2)}=\frac{\mp \sqrt{1-h^{2}}+\cos (2 \psi)}{h+\sin (2 \psi)},
$$

and the quantity $h=q_{Y}^{\prime}(p)$ is a generalised pressure modulus. Note that in this expression $p$ must be critical, i.e., equal to its yield value. For incompressible materials $h=0$.

Rescaling (2-1) and (2-2) along the characteristics, Equation (3-1) provides

$$
r_{i}^{(k)} B_{i j} \frac{\partial(p, \psi)}{\partial s}=0 \quad(k=1,2),
$$


where the left eigenvectors are

$$
r^{(1,2)}=\left(\frac{\sqrt{1-h^{2}} \mp \cos (2 \psi)}{ \pm(h-\sin (2 \psi))}, 1\right) .
$$

Equations (3-3) are derived in Appendix B and expanding them yields the generalised Henchky's equations

$$
0=\sqrt{1-h^{2}} p_{s} \pm 2 q \psi_{s}
$$

In the case of a von Mises material, where $q_{Y}=k$ is a constant, (3-2) simplifies (as $h=0$ ) to the familiar form

$$
\mu^{(1)}=-\tan \psi, \quad \mu^{(2)}=\cot \psi
$$

and the eigenvectors (3-4) to

$$
r^{(1)}=(-\tan \psi, 1), \quad r^{(2)}=(\cot \psi, 1)
$$

The corresponding traces are commonly known as $\alpha / \beta$-slip lines. Along the slip lines Equations (3-5) reduce to the classical Hencky's equations

$$
p \pm 2 k \psi=C_{\alpha, \beta}
$$

3B. Generalised Geiringer's equations. Traditionally the velocity components along the slip lines are described by Geiringer's equations which are obtained from the continuity equation, in conjunction with the plastic flow rule and the small-strain compatibility equations [Hill 1950]. In the classical isothermal formulation of slip line field theory velocity solutions are sought that satisfy the continuity equation and localisation of plastic deformation is defined as a velocity jump across the slip line (velocity discontinuity) of zero thickness. In the present formulation we relax the isothermal assumption and seek conditions of localisation that replace the vanishing thickness by a finite width.

To this end, the mechanical dissipation $\Phi$ of (2-5) is combined together with the constitutive law of plasticity (2-8) to provide a temperature sensitive dissipation term

$$
\Phi=\beta \sigma_{i j} \dot{\varepsilon}_{i j}^{p}=\beta \dot{\varepsilon}_{0} \sigma_{i j}\langle f(g)\rangle e^{-T_{0} / T} \frac{\partial g}{\partial \sigma_{i j}},
$$

where the term $\sigma_{i j}\left(\partial g / \partial \sigma_{i j}\right)$ represents a tensorial product. The stability and bifurcation of the energy balance equation (2-4) is characterised by the nonlinear response of its steady state. This problem has been extensively studied in the literature for shear zones [Gruntfest 1963; Chen et al. 1989; Leroy and Molinari 1992; Vardoulakis 2002; Veveakis et al. 2010]. In this work we generalise it for 2D loading conditions by first studying the response of the energy balance in the original geometry and then rescaling it to a one-dimensional subspace defined by the directions of the characteristics.

3B.1. Steady state analysis in the original geometry. The steady state of the energy balance equation is defined by

$$
\alpha\left(\frac{\partial^{2}}{\partial x_{1}^{2}}+\frac{\partial^{2}}{\partial x_{2}^{2}}\right) T+\beta \dot{\varepsilon}_{0} \sigma_{i j}\langle f(g)\rangle \frac{\partial g}{\partial \sigma_{i j}} e^{-T_{0} / T}=0
$$


The energy balance can be brought into dimensionless form by setting

$$
\theta=\frac{T-T_{b}}{T_{b}}, \quad \hat{x}_{i}=\frac{x_{i}}{L_{i}} \quad(i=1,2), \quad \operatorname{Ar}=\frac{T_{0}}{T_{b}},
$$

where $T_{b}$ is the boundary temperature and $L_{i}$ is an appropriate length scale. Since we are interested in deformation taking place under isothermal boundary conditions the final dimensionless equation is (the superimposed hats are dropped for convenience)

$$
\left(\frac{\partial^{2}}{\partial x_{1}^{2}}+\lambda^{2} \frac{\partial^{2}}{\partial x_{2}^{2}}\right) \theta+\mathrm{Gr}^{2 \mathrm{D}} \exp \left(\frac{\operatorname{Ar} \theta}{1+\theta}\right)=0,
$$

where $\lambda=L_{1} / L_{2}$ is an aspect ratio and the superscript $2 \mathrm{D}$ refers to the dimension of the domain. The exponential term stems from the mechanical dissipation $\Phi$ and we define the normalised dissipation function

$$
\phi=\exp \left(\frac{\operatorname{Ar} \theta}{1+\theta}\right)
$$

In order to solve the partial differential equation (3-12) on the domain $[-1,1] \times[-1,1]$ a pseudo arclength continuation [Chan and Keller 1982] in $\mathrm{Gr}^{2 \mathrm{D}}$, the Gruntfest number [Gruntfest 1963], was carried out where

$$
\mathrm{Gr}^{2 \mathrm{D}}=\frac{\beta \dot{\varepsilon}_{0} L_{1}^{2}}{\alpha T_{b}} \sigma_{i j}\langle f(g)\rangle e^{-\mathrm{Ar}} \frac{\partial g}{\partial \sigma_{i j}},
$$

and (3-12) is subjected to isothermal boundary conditions

$$
\theta( \pm 1, y)=0, \quad \theta(x, \pm 1)=0
$$

In physical terms, Gr represents the ratio between heat production due to mechanical deformation and heat loss due to thermal conduction or additional energy sinks [Vardoulakis 2002; Veveakis et al. 2010]. At the low Gr limit the system deforms in virtually isothermal conditions, whereas as $\mathrm{Gr} \rightarrow \infty$ so the system deforms under near-adiabatic conditions [Veveakis et al. 2010].

It is well known that the steady state response of the system depends on the values of Gr and Ar [Law 2006; Veveakis et al. 2010], see Figure 1. In Figure 1(a) we sketch the maximum temperature at the centre of the domain, $\theta_{c}$, as a function of $\mathrm{Gr}$ for two values of Ar. We notice that between $\mathrm{Ar}=4$ and $\mathrm{Ar}=5$ the response of the system changes from a stretched $(\mathrm{Ar}=4)$ to a folded $\mathrm{S}$-curve $(\mathrm{Ar}=5)$. The stability of the system is determined by the eigenvalues of the Jacobian matrix, shown in Figure 1(b). Since the maximum eigenvalue of the case $\mathrm{Ar} \leq 4$ (stretched) is negative for all values of Gr, the stretched S-curve is stable throughout. In contrast, the folded $\mathrm{S}$-curve $(\mathrm{Ar}=5)$ exhibits two points of stability change, coinciding with the turning points of the S-curve. This means that the stationary solutions are initially stable up to the first turning point, then unstable and after the second turning point restabilise.

The above arguments are independent of $\lambda$ and the variation of the steady state response with respect to $\lambda$ are presented in Figure 2. This conclusion allows us to proceed with rescaling the energy equation in a $1 \mathrm{D}$ equivalent, along the characteristic curves $s_{k}$ of the stress equilibrium equations. 

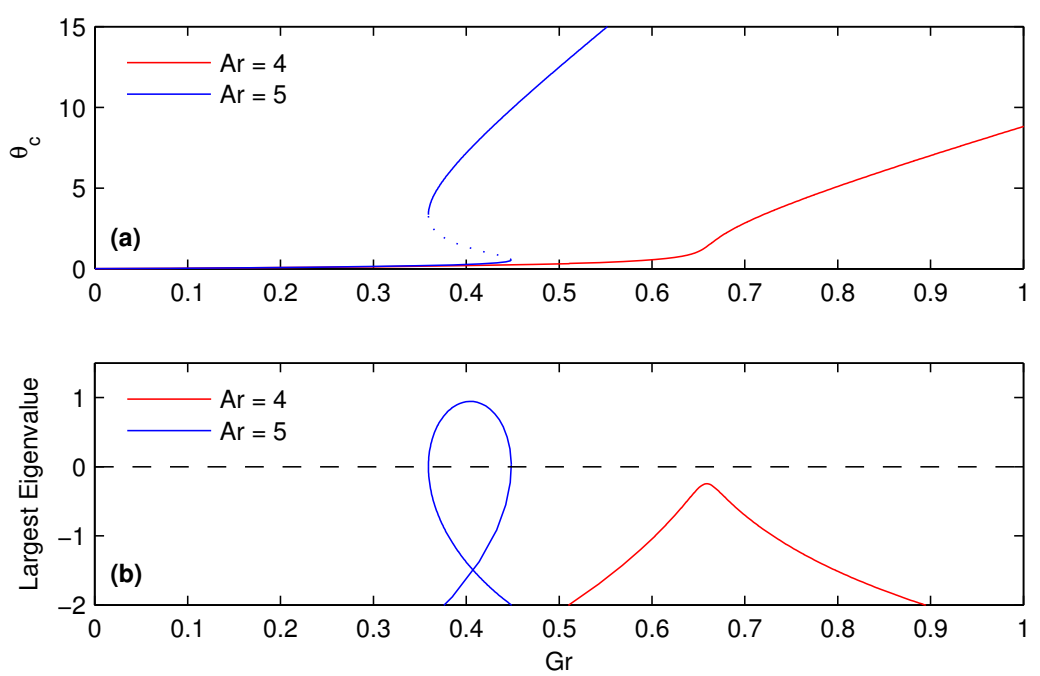

Figure 1. (a) Bifurcation diagram of the two-dimensional heat equation for $\mathrm{Ar}=4,5$. If the path of stationary states is folded $(\mathrm{Ar}=5)$ the solutions change stability at the fold and dotted lines denote unstable stationary states. (b) Largest eigenvalues of the Jacobian matrix associated with (3-12). For $\mathrm{Ar}=4$ the largest eigenvalue remains negative, but for $\mathrm{Ar}=5$ stability of the stationary solutions changes at the folds where the largest eigenvalue changes sign.
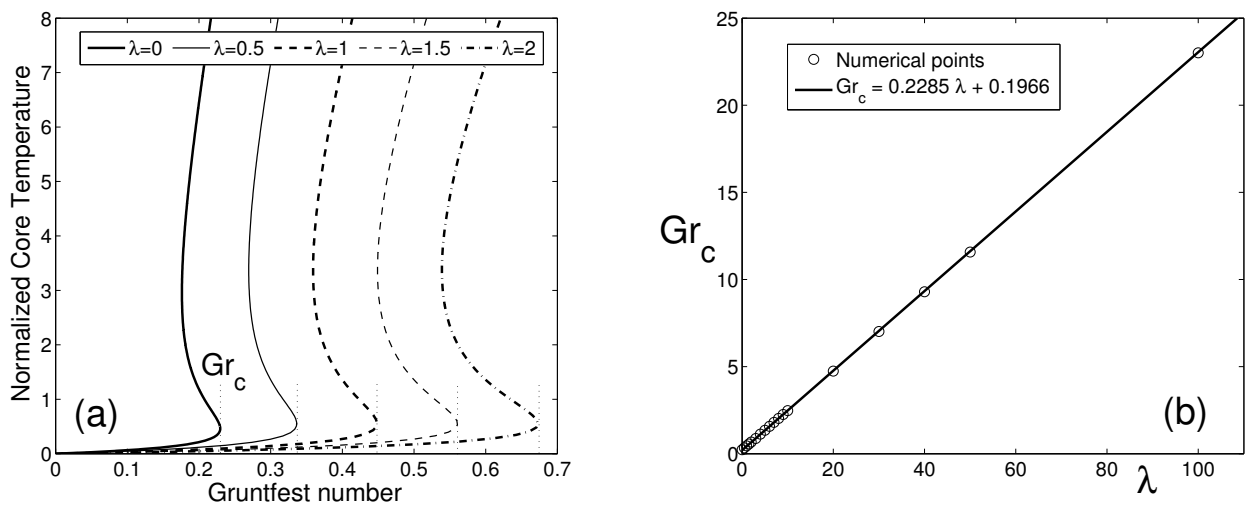

Figure 2. A change in aspect ratio $\lambda$ shifts the steady states along the Gr-axis, but has only little influence on the shape of the S-curve and no influence on the stability of the steady states.

3B.2. Rescaling along the characteristics. In this work we look for the necessary conditions for localisation of the plastic deformation to occur along the characteristics of the stress equilibrium. This means that the characteristic curves $s_{k}$ also need to be the characteristics of the temperature equation. This condition is satisfied in the limit where the elliptic operator vanishes, which is also the adiabatic limit. 
Hence, the loss of ellipticity of the temperature equation would ensure that localisation is achieved along the characteristics of the momentum equation. Localisation should therefore be obtained through the properties of the nonlinear response of the energy equation.

Replacing the spatial derivatives in (3-10) with (3-1) yields

$$
\alpha \frac{\left(1+\mu^{2}\right)}{\mu^{2}}\left(\left(1+\mu^{2}\right) \frac{\partial^{2}}{\partial s^{2}}+\frac{\left(\mu^{2}-1\right)}{\mu} \frac{\partial \mu}{\partial s} \frac{\partial}{\partial s}\right) T+\beta \dot{\varepsilon}_{0} \sigma_{i j}\langle f(g)\rangle \frac{\partial g}{\partial \sigma_{i j}} e^{-T_{0} / T}=0,
$$

and all super- and subscripts $k$ have been dropped since the above arguments hold true for both sets of characteristics. We consider the case of infinitesimal variations of the slip line geometry, so that the derivatives $\partial \mu / \partial s$ are small and can be ignored.

Analogously to (3-11), (3-16) is normalised and

$$
\frac{\partial^{2} \theta}{\partial s^{2}}+\mathrm{Gr}^{1 \mathrm{D}} \exp \left(\frac{\operatorname{Ar} \theta}{1+\theta}\right)=0
$$

where the Gruntfest number admits the following form

$$
\mathrm{Gr}^{1 \mathrm{D}}=\frac{\beta \dot{\varepsilon}_{0} L^{2}}{\alpha T_{b}} \frac{\mu^{2}}{\left(1+\mu^{2}\right)^{2}} e^{-\mathrm{Ar}} \sigma_{i j}\langle f(g)\rangle \frac{\partial g}{\partial \sigma_{i j}},
$$

where $L$ is a length scale. This Gruntfest number is spatially dependent through $\mu$, and also incorporates the dimensionality of the system at hand, through $L$ and we conjecture that the critical values of the Gruntfest number for the two dimensional and one dimensional case are related via

$$
\mathrm{Gr}_{\mathrm{cr}}^{1 \mathrm{D}}=\frac{L^{2}}{L_{1}^{2}} \frac{\mu^{2}}{\left(1+\mu^{2}\right)^{2}} \mathrm{Gr}_{\mathrm{cr}}^{2 \mathrm{D}},
$$

which follows upon comparing (3-14) and (3-18). As an example, we consider the experiment in [Johnson et al. 1964] where the heat lines are oriented along the diagonals of a square sample. When rescaling a von Mises material along the characteristics of a square domain (see the next section) with dimension $L_{1}$, then $L=\sqrt{2} L_{1}$ and the geometric correction

$$
\frac{L^{2}}{L_{1}^{2}} \frac{\mu^{2}}{\left(1+\mu^{2}\right)^{2}}=\frac{1}{2}
$$

as $\mu=-\tan \psi$ or $\mu=\cot \psi$. From here on forward we do not distinct between the $\mathrm{Gr}^{1 \mathrm{D}}$ and $\mathrm{Gr}^{2 \mathrm{D}}$ any longer since there is no dis-ambiguity in terms of the physics between the one- and two-dimensional heat equation.

The steady state response of this $1 \mathrm{D}$ equivalent equation follows that of the $2 \mathrm{D}$ case, as shown in Appendix C. In Figure 3, we present the results of this analysis for the dissipation function $\phi$ and we find three distinct steady states of alternating stability. The unstable branch BC corresponds to a localisation instability across the characteristic trace for the normalised dissipation function $\phi$. Since in Figure 3(a) the branches $\mathrm{AB}$ and $\mathrm{CD}$ are stable in the dissipation the unstable branch $\mathrm{BC}$ cannot be admitted and the solution jumps from stable near isothermal homogeneous plastic deformation on $\mathrm{AB}$ to localised near adiabatic deformation on $\mathrm{CD}$. The saddle point $\mathrm{B}$ therefore corresponds to the necessary condition for the loss of ellipticity of the thermomechanical system, after which localisation is progressively achieved. 

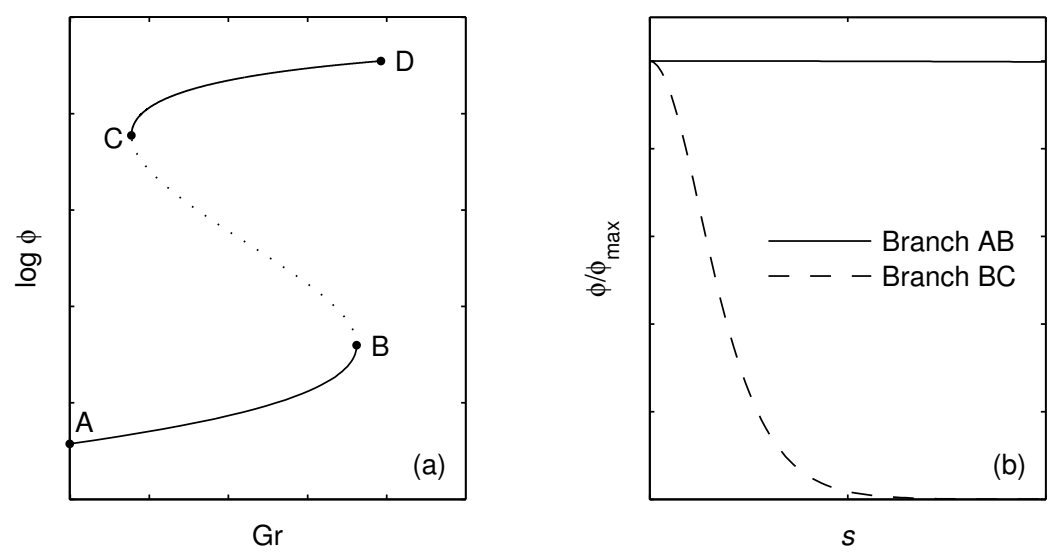

Figure 3. (a) Folded S-curve. Along the branch AB the solution of (3-12) corresponds to an isothermal temperature profile whereas along the section between the turning points $\mathrm{B}$ and $\mathrm{C}$ the solutions localise. (b) Examples of the one-dimensional spatial pattern of the dissipation profile for $\mathrm{Ar}=10$. The profiles are normalised with respect to the maximum value of dissipation.

It is a similar condition to the traditional jump conditions for stress and velocity discontinuities in the classical slip line field solution but is expressed here in terms of their product, the dissipation.

This transition from homogeneous to localised deformation is shown in Figure 3(b), where the normalised dissipation is shown to localise towards the centre of the domain as it crosses the unstable BC branch of the S-curve (see also the results of [Leroy and Molinari 1992; Veveakis et al. 2010]). The critical threshold for the transition from the stable branch $\mathrm{AB}$ to the new steady state $\mathrm{CD}$ is characterised by the turning point $\mathrm{B}$ and can be calculated asymptotically as shown in the Appendix C and Figure 10.

\section{Transient analysis of the system}

The steady state limit characterises the long-term behaviour of the system, but the evolution of the system given a certain initial condition can only be obtained through direct time integration of the transient system of equations of a von Mises elasto-(visco-)plastic material:

$$
\frac{\partial \sigma_{i j}}{\partial x_{i}}=0, \quad \rho c \frac{\partial T}{\partial t}=\alpha \frac{\partial^{2} T}{\partial x_{i}^{2}}+\beta s_{i j} \dot{e}_{i j}^{p}, \quad \dot{e}_{i j}=\frac{s_{i j}}{2 G}+\dot{\varepsilon}_{0}\left(\frac{\sqrt{q}}{k}-1\right) \frac{s_{i j}}{q} e^{-T_{0} / T} .
$$

where $G$ is the elastic shear modulus, and the last term of the third equation corresponds to the viscoplastic contribution of an associative von Mises material. Notably, although the momentum balance is at its stationary limit, the time evolution of the system is achieved through the temperature equation. The energy balance therefore acts as a postfailure evolution equation similar to the ad hoc evolution laws of the hardening modulus in classical plasticity.

In this section, we integrate this system of equations for the case of an ideal viscoplastic $(G \rightarrow \infty)$ and an elasto-viscoplastic material with thermomechanical coupling. To illustrate the results of the steadystate analysis an elementary problem set is chosen, where in a plane strain setting the loading conditions shown in Figure 4 are applied. A square cell is pinned in the $x, y$-direction on the left hand side and in 


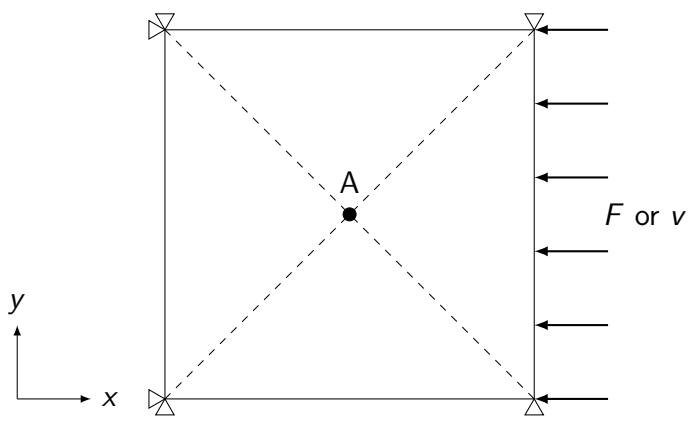

Figure 4. Geometry of the problem configuration. A square is deformed by applying either a constant force $F$ or constant velocity $v$ at the right hand side of the sample. The sample is pinned on the left and in the $y$-direction. At the centre "A" the sample is probed for various quantities. The theoretical analysis presented in the previous sections for a rate-dependent von Mises material suggests that the slip lines of this model run diagonally across the specimen and are represented by dashed lines [Johnson et al. 1964].

the $y$-direction on the right hand side. It is loaded on the right boundary with either a constant force $F$ or constant velocity $v$. The cell is surrounded by a reservoir that has a constant temperature $T_{b}$.

The results of the previous sections suggest that in such a configuration the slip lines should propagate from the pinned corners of the rectangle, diagonally across the specimen as discussed in [Johnson et al. 1964]. Owing to the pressure independent von Mises yield envelope, the slip lines are expected to be perpendicular to each other. In addition, the critical condition for the localisation of plastic deformation is expected to be given by the critical value of the Gruntfest number, as shown in the previous sections. In the time-dependent case, we may follow the same rules of normalisation ((3-11)) and define the Gruntfest number as

$$
\mathrm{Gr}=\left(\frac{\beta \dot{\varepsilon}_{0} L^{2}}{4 \alpha T_{b}} e^{-\mathrm{Ar}}\right) s_{i j}\left\langle\frac{q}{k}-1\right\rangle .
$$

Because the Gruntfest number incorporates the mechanical work, we expect the Gruntfest analysis of the previous section to hold for both constant force and constant velocity conditions. The difference between the two regimes would be that in the case of constant force conditions Gr will obtain a constant value, once the stress equilibrates to a constant value. Under constant velocity conditions, the stress, and so Gr, will continue to increase throughout the time of loading. Therefore, given enough time the system will always be able to cross the critical Gr value for the onset of localisation.

In the following sections we present the results of the time-integration of the system (4-1). The analysis was carried out using finite element techniques as implemented in the finite element code REDBACK ${ }^{1}$ in which the viscoplasticity is solved using Perzyna's overstress formulation. REDBACK is an open source application derived from the Multiphysics Object Oriented Simulation Environment (MOOSE) [Gaston et al. 2009]. MOOSE provides a simulation environment that is focussed on the physics of the problem at hand and it takes care of meshing and numerical handling of memory and structures internally via the finite element library libMesh [Kirk et al. 2006] and the nonlinear parallel solver PETSc

\footnotetext{
${ }^{1}$ Available at http://github.com/pou036/redback.git.
} 
[Balay et al. 2014], respectively. Hence, MOOSE's approach to the finite element method is mesh and dimension independent which differentiates it from other solvers that require the user to define elements and handle more in-depth numerical issues. MOOSE is based in Jacobian-free Newton-Krylov methods for computational efficiency [Knoll and Keyes 2004].

Initially we present two cases for the deformation of an ideal viscoplastic material and an elastoviscoplastic material under constant force boundary conditions. Further, the response of the elastoviscoplastic material under a constant velocity boundary condition is studied.

4A. Ideal viscoplastic material under constant force boundary conditions. In the case of an ideal viscoplastic material $(G \rightarrow \infty)$ under constant force conditions the stress exerted onto the sample is constant throughout the simulation and the sample enters the plastic regime immediately. Hence, Gr is held constant throughout the loading. The evolution of the deforming system is best represented by its orbits in $\mathrm{Gr}-\theta$ space as shown in Figure 5 because this allows for a direct comparison between the "S-curve" that marks the steady states in Gr- $T$ space and the steady state attained by the sample. As $\mathrm{Gr}$ is constant these orbits are vertical lines in the case of ideal viscoplasticity. Figure 5 summarises the evolution of the deforming sample for various initial conditions (marked by grey squares) and their final state (black squares). The results of these simulations confirm the concept of the "S-curve" that is comprised of two stable branches that are linked by an unstable branch (BC). If $\mathrm{Gr}$ is chosen to be larger than the critical value (turning point B) or the initial state lies above the unstable branch BC the system converges to the high-energy (temperature), adiabatic branch. On the other hand, if $\mathrm{Gr}$ is smaller than the critical value the system converges to the low-energy (temperature) isothermal branch.

When elasticity is incorporated and the system is loaded using constant force, the Gruntfest number (i.e., the overstress) is expected to relax to a constant value after its initial evolution due to the elastic

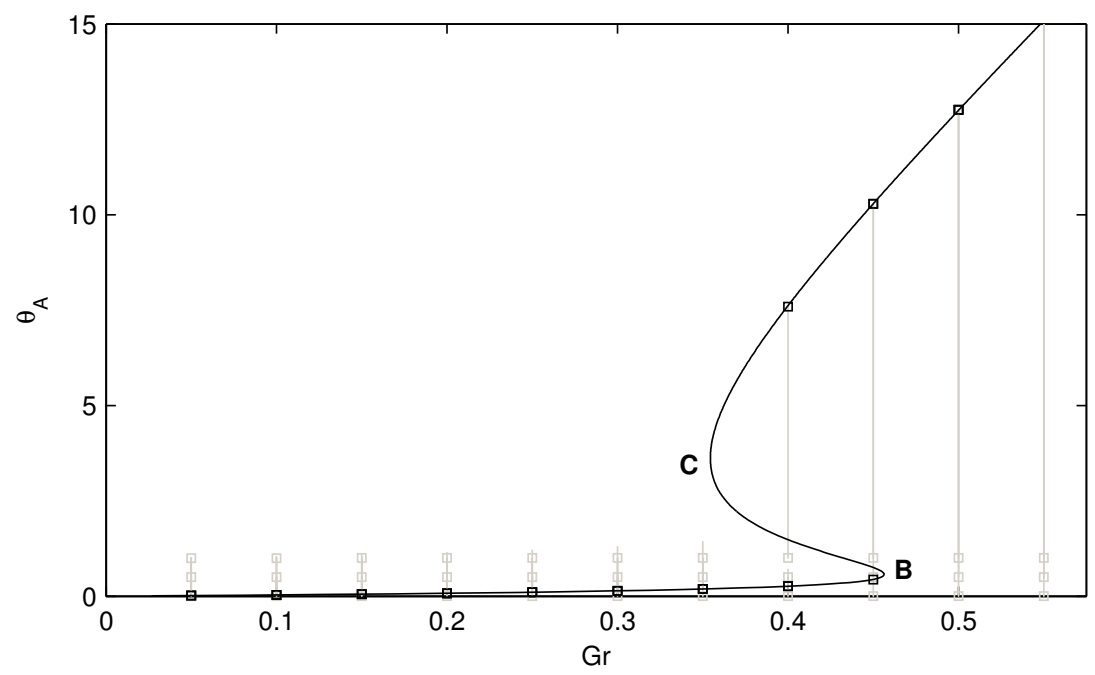

Figure 5. Evolution of an ideal viscoplastic material that is deformed with a constant force. The solid black line marks steady states. The grey lines represent the trajectories through the Gr- $\theta$ space as the system evolves in time. Initial and final conditions are marked by grey and black squares, respectively. 
response of the system. This regime allows us to test the extent of the validity of the S-curve concept established in Section 3B, since the orbits of the elasto-viscoplastic material are not constant in Gr. To this end, the system of equations (4-1) was integrated for different initial conditions and values of Gr. The results are summarised in Figure 6(a) where we observe that the phase space is indeed split into two domains, in accordance with the concept of the S-curve. Grey squares denote initial conditions and black squares denote the final state of each numerical experiment.

For initial conditions starting below the unstable branch (branch BC of Figure 3) the system evolves towards a low-energy steady state, which coincides with the stable branch AB of Figure 3. Correspondingly, for initial conditions above the unstable branch the orbits diverge and eventually relax in a high-energy steady-state where dissipation localises along the characteristics of the stress equilibrium equations. This high energy steady state corresponds to the branch CD of Figure 3. Runs that started very close to, but above, branch BC (solid black squares) diverge towards the high temperature branch as well. The time evolution of these orbits showcase the different responses (Figure 6(b,c)). Indeed, the low-energy orbits converge quickly to the low-energy steady state where plastic deformation is not localised, whereas the high-energy orbits provide an evolution of the system towards the localisation of dissipation along the characteristics of the stress equilibrium equations. This transition is equivalent to the evolution from secondary to tertiary creep in material science (Figure 6(b,c)).

4B. Elasto-viscoplastic material under constant force boundary conditions. Following the results of Figure 3, we expect the orbits leading to the low-energy steady state to be stable without profound localisation of the plastic deformation. On the contrary, the high-energy steady-state is expected to provide the necessary conditions for localisation. In Figure 7 we plot the spatial distribution of the mechanical dissipation for an orbit converging to a low-energy and high-energy steady-state. We notice that in both cases the slip lines indeed propagate from the corners towards the diagonals of the specimen as expected, but that in the low-energy case the plastic deformation is not localised across the slip lines, whereas in the high energy case localisation is emerging, with localisation being more pronounced the higher the value of Gr. We may therefore suggest indeed that the critical Gruntfest number constitutes a material bifurcation criterion for temperature sensitive viscoplastic materials.

4C. Heat lines under constant velocity boundary conditions. When the elasto-viscoplastic material is loaded at constant velocity conditions, the Gruntfest number initially grows rapidly and attains values beyond its critical value. Hence, the temperature rises rapidly and the system can be assumed to transition quickly from near-isothermal to near-adiabatic conditions.

An extreme end-member of this regime is the case of an elasto-viscoplastic material evolving under fast loading, thus establishing directly adiabatic conditions. This regime which is also known as adiabatic shear banding is crucial in metal forming and cutting [Gruntfest 1963], but also in geomaterials [Vardoulakis 2002]. In the present study adiabatic shear banding constitutes just a limiting case of the analysis, when $\mathrm{Gr} \rightarrow \infty$. Such a case can be simulated by setting the thermal diffusion to zero in the temperature equation which is equivalent to assuming that the diffusive capabilities of the material are negligible as compared to the heat producing capabilities. In this case, the dimensionless form of the temperature equation (4-1) reads

$$
\frac{\partial \theta}{\partial t_{\mathrm{ad}}}=\exp \left(\frac{\theta}{1+\epsilon \theta}\right),
$$



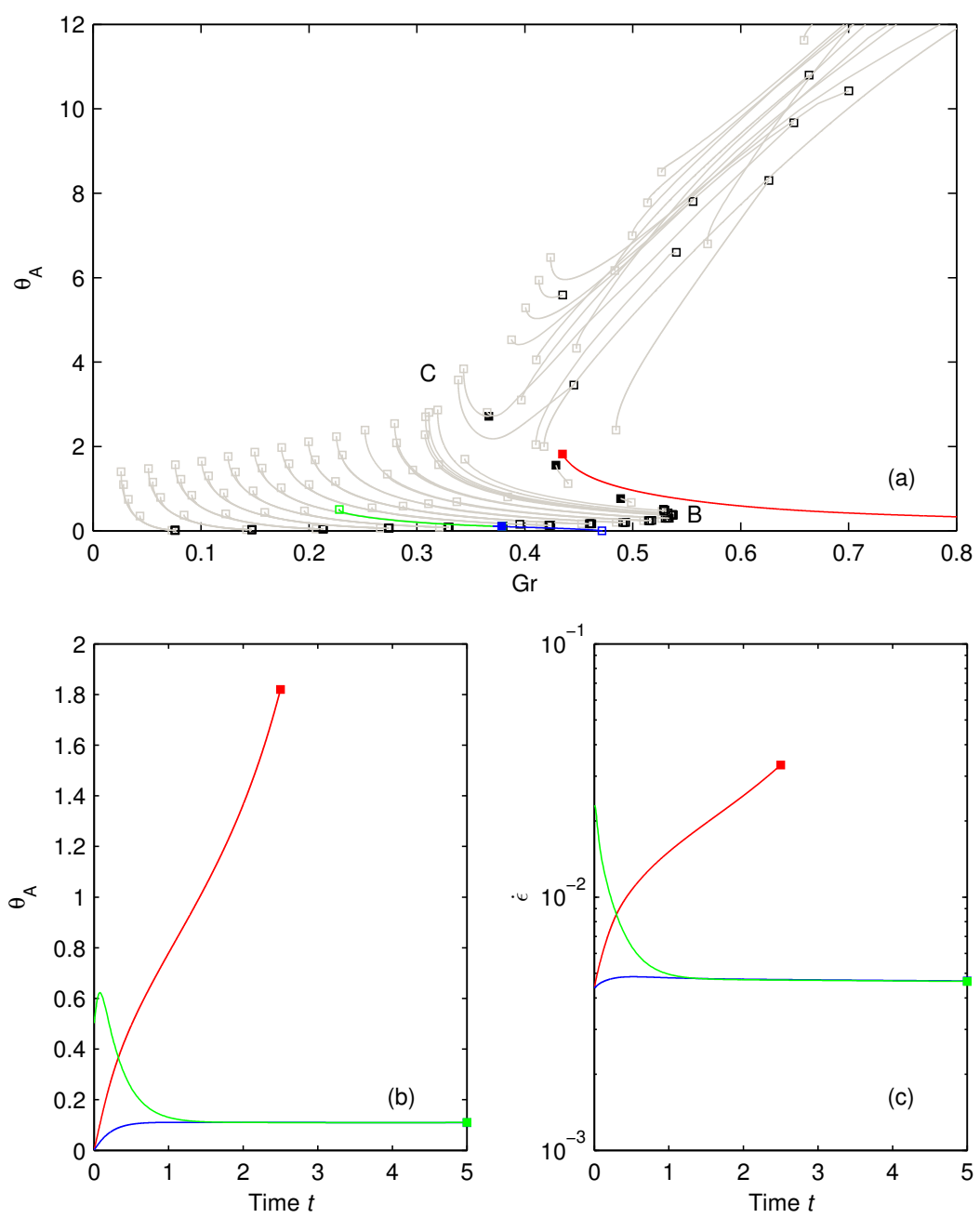

Figure 6. (a) Evolution of an elasto-viscoplastic material deformed with a constant force. Grey and black squares mark initial and final states of the system of the simulation, respectively. The solid black squares mark the final state of runs with initial conditions very close to but above the separatrix $\mathrm{BC}$ and we find that the these runs are indeed intrinsically unstable, blowing up in short times. (b) Centre temperature $\theta_{A}$ over time for the three runs marked in (a). (c) Strain rate $\dot{\varepsilon}$ at point $A$ over time for the three runs marked in (a).

where $t_{\mathrm{ad}}=\mathrm{Gr} \cdot t$ is finite only when $t \rightarrow 0$, since $\mathrm{Gr} \rightarrow \infty$. This means that the limiting case of adiabatic shearing takes place in explosive timescales of the order $t \sim \mathcal{O}(1 / \mathrm{Gr})$ [Gruntfest 1963; Veveakis et al. 2007]. Hence, the Gruntfest number Gr controls the time evolution of the adiabatic shear banding, in addition to the onset of localisation.

Under such a scenario the localisation of dissipation along the slip lines is profound, and patterns resembling the heat lines of [Johnson et al. 1964] are obtained, as shown in Figure 8. In this case the system enters directly the unstable area of the S-curve, and traces the upper branch CD during the time of loading. 

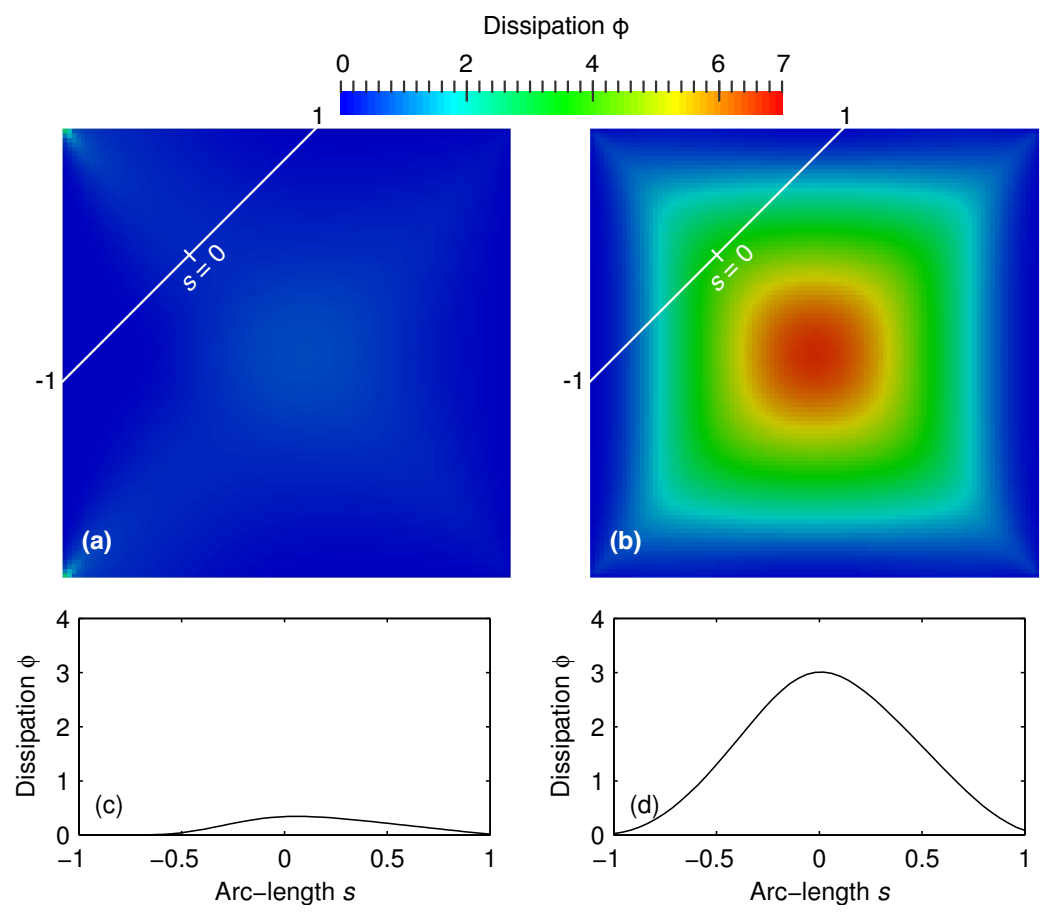

Figure 7. Dissipation profiles for the final state of a run that converges towards the low temperature branch $\mathrm{AB}$ and a run that converges towards branch $\mathrm{CD}$. The spatial distribution of the mechanical dissipation: (a) isothermal low temperature stable branch without significant localisation of plastic deformation. (b) simulation for the high temperature branch with significant localised heating. The dissipation profiles were recorded along the lines highlighted in (a) and (b) and are presented in (c) and (d), respectively.
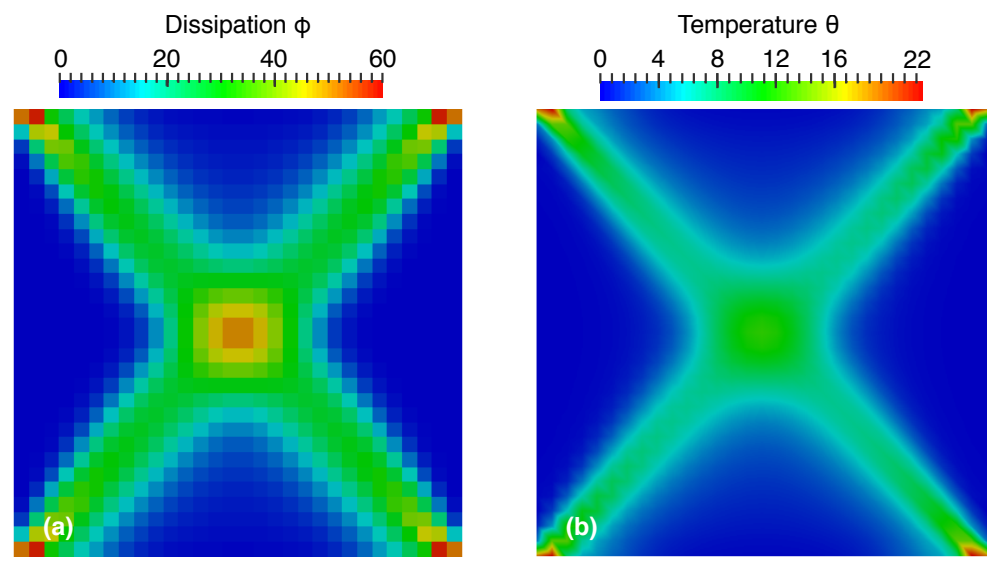

Figure 8. Heat lines under fast constant velocity loading. The heat lines emerge from an initially homogeneous temperature state. The heat lines follow the slip lines and can be observed as a localisation of (a) mechanical dissipation and (b) temperature. 


\section{Discussion}

The simulation of the temporal evolution of temperature and rate-sensitive materials under various loading conditions verifies the results of the steady-state response. In conclusion, the problem of mechanical deformation of a temperature-sensitive viscoplastic material can be elegantly captured by the bifurcation curves of Figure 1. We have seen that for this class of materials the energy equation determines the conditions for the onset of localisation of plastic deformation, which occurs along the characteristics of the stress equilibrium equations.

The results of Figure 6 highlight this energy bifurcation as the main driver of the transient orbits in the system, even when elasticity is considered. It therefore provides a material bifurcation criterion and, combined with the analysis of Section 3A, a generalisation of the slip line field theory for thermoviscoplastic materials. The main effect of an extension to rate and temperature dependence is that lines of velocity discontinuity do not exist below a critical temperature and deformation rate corresponding to a critical dissipation as captured by the Gruntfest number Gr. Above critical Gr the classical slip lines emerge in terms of velocity discontinuities and below critical Gr homogeneous deformation is derived.

The extension of the analytical treatment of generalised slip line field theory to the transient regime using a numerical scheme has allowed us to test it for the problem of metal forging [Johnson et al. 1964]. The constant velocity boundary condition applied during forging lead to a variation in Gr thereby crossing the stability regimes from homogeneous deformation to the appearance of heat lines. The fast loading during this deformation can lead the material directly to the extreme case of adiabatic deformation.

This work has therefore presented a generalised approach of slip line field theory showing that the simple, but restrictive, rheology of a rigid-plastic body can be extended to included rate dependent and temperature dependent material behaviour. We have detailed an energy based framework to obtain the modified Hencky's and Geiringer's equations which still provide the information on the basic pattern of slip lines underpinning the deformation process. We argue that this extension is therefore useful for gaining a basic understanding into the more complex material behaviour from an analytical perspective. The extension of the analytical approach reveals, however, a more complex material behaviour than in the classical theory.

The results of the present study highlight the role of the energy balance in material bifurcations. Since the critical condition for material instability is retrieved from an energy bifurcation, the temperature equation can act as a hardening law and substitute the experimentally derived hardening laws. Such a case would allow us to account explicitly for different physics as well as for the mechanisms acting at different scales in a material through their energy budget. We are therefore one step further to our quest on multiscale analyses, since all the necessary mechanisms can be explicitly accounted for in the energy equation through the corresponding internal state variables of the microprocesses. The results of the present work can provide the basis for a unified theory for material behaviour, starting from solid mechanics and extending naturally to the fluid-like postfailure evolution of materials using explicitly the energy considerations as the link.

\section{Appendix A: The complete energy budget}

A consistent description of the thermomechanical behaviour of viscoplastic materials must be based on the conservation of (1) mass, (2) momentum and (3) energy. In addition to these conservation laws 
it must be ensured that the entropy production is positive at all times. The combination of these four prerequisites leads to the heat equation

$$
\rho c \dot{T}=\partial_{x_{i}} \alpha \partial_{x_{i}} T+\Phi+r+T\left(\partial_{T} \sigma_{i j}\right) \dot{\varepsilon}_{i j}^{e}
$$

that describes the local energy budget and where $\rho$ denotes the material density, $c$ the specific heat, $\alpha$ the heat conductivity, $\Phi$ the mechanical dissipation, $\sigma_{i j}$ the stress tensor, $\dot{\varepsilon}_{i j}^{e}$ the elastic strain rate. The significance of this equation is that it couples the temperature evolution to the mechanical properties of the material.

In the context of thermomechanical coupling the term $r$ in (A-1) could be thought of as a heat sink that has the effect of limiting temperature production after the onset of localisation. Possible heat sinks due to a postlocalisation transformation are melting or endothermic chemical reactions [Rosakis et al. 2000]. Without the heat sink uncontrolled thermal runaway would ensue and hence $r$ acts as a stabiliser [Veveakis et al. 2010]. In this work $r$ is neglected, because the aim of this work is to find criteria for the onset of localisation and a detailed study of the postlocalisation regime is not of central interest. In doing so, the postfailure evolution of temperature will be exaggerated, since the heat-absorbing processes gathered in $r$ do not limit the temperature evolution.

The final term in (A-1) describes thermoelastic heating, but is ignored in this work for simplicity of the mathematical treatment.

\section{Appendix B: Characteristic traces and generalised Hencky equations}

Here, the stress equilibrium equations (2-1) and (2-2) are rewritten as a system of first-order differential equations by means of the method of characteristics and this process reveals the characteristic traces inherent to the stress equilibrium equations. In the context of plasticity characteristic traces are often referred to as slip lines along which failure will occur [Hill 1950]. Hence, in the remainder of this section the geometry of the failure patterns is determined.

Rather than solving equations (2-1) and (2-2) for the stress components $\sigma_{i j}$ themselves we employ the Mohr circle transformation (2-3) and evaluate $p, q$ and $\phi$. Since there are three unknowns and only two equations a further relation is required and we choose a general yield criterion that allows to express $q$ in terms of $p, q=q(p)$.

After substituting the stress components (2-3), (2-1) and (2-2) can be conveniently written in matrix form as

$$
A_{i j} \frac{\partial(p, \psi)}{\partial x_{1}}+B_{i j} \frac{\partial(p, \psi)}{\partial x_{2}}=0
$$

where

$$
\begin{aligned}
& A=\left(\begin{array}{cc}
1-q^{\prime} \sin (2 \psi) & -2 q \cos (2 \psi) \\
q^{\prime} \cos (2 \psi) & -2 q \sin (2 \psi)
\end{array}\right), \\
& B=\left(\begin{array}{cc}
q^{\prime} \cos (2 \psi) & -2 q \sin (2 \psi) \\
q^{\prime} \sin (2 \psi)+1 & 2 q \cos (2 \psi)
\end{array}\right),
\end{aligned}
$$


and the prime $(\cdot)^{\prime}$ denotes differentiation with respect to $p$. In order to simplify (B-1) the two (left) eigenvectors $r_{i}$ and eigenvalues $\mu$ that satisfy

$$
r_{i}^{(k)} A_{i j}=\mu^{(k)} r_{i}^{(k)} B_{i j} \quad(k=1,2)
$$

are computed and (B-4) is substituted into (B-1) so that

$$
r_{i}^{(k)} B_{i j}\left(\frac{\mu^{(k)}}{\sqrt{1+\mu^{(k) 2}}} \frac{\partial(p, \psi)}{\partial x_{1}}+\frac{1}{\sqrt{1+\mu^{(k) 2}}} \frac{\partial(p, \psi)}{\partial x_{2}}\right)=0 .
$$

Instead of solving (B-5) on the whole $x_{1}-x_{2}$ plane we restrict ourselves to one characteristic trace along which the independent variables $x_{1}$ and $x_{2}$ can be parametrised in terms of the arc-length $s$. Parametrising along the characteristic and identifying

$$
\frac{\partial x_{1}}{\partial s_{k}}=\frac{\mu^{(k)}}{\sqrt{1+\mu^{(k) 2}}}, \quad \frac{\partial x_{2}}{\partial s_{k}}=\frac{1}{\sqrt{1+\mu^{(k) 2}}}
$$

allows us to write (B-5) as

$$
r_{i}^{(k)} B_{i j} \frac{\partial(p, \psi)}{\partial s_{k}}=0
$$

and the coupled system of ordinary differential equations (B-6) and (B-7) is equivalent to stress equilibrium equations (2-1)-(2-2) along the characteristic.

We wish to emphasise that (B-6) defines the geometry of the characteristic trace and that there are two characteristic traces. In general, the eigenvalues $\mu^{(k)}$ depend on $\psi$ and $q^{\prime}(p)$.

\section{Appendix C: Asymptotic approximations}

In this section asymptotic solutions to the nondimensional heat equation (3-12) are computed, but the perturbation analysis requires us to identify a "small" parameter which is used as the perturbation parameter. If the temperature is measured in units of Ar such that $\tilde{\theta}=\operatorname{Ar} \theta$ the heat equation (3-16) reads

$$
\tilde{\theta}^{\prime \prime}+\widetilde{G r} \exp \left(\frac{\tilde{\theta}}{1+\varepsilon \tilde{\theta}}\right)
$$

where $\widetilde{G r}=\operatorname{Ar}$ Gr and $\varepsilon=1 / \operatorname{Ar} \ll 1$ and $\varepsilon$ is a suitable perturbation parameter. We drop the $\sim$ superscript for convenience.

If $\theta \sim \mathcal{O}(1), \varepsilon \theta \ll 1$ and the exponent in (C-1) can be expanded as a geometric series which yields

$$
\theta^{\prime \prime}+\operatorname{Gr} \exp \left(\theta-\varepsilon \theta^{2}+\varepsilon^{2} \theta^{3}+\cdots\right)=0 .
$$

In order to find the governing equations at increasing orders of $\varepsilon$ we expand

$$
\theta=\theta_{0}+\varepsilon \theta_{1}+\cdots \quad \text { and } \mathrm{Gr}=\mathrm{Gr}_{0}+\varepsilon \mathrm{Gr}_{1}+\cdots
$$

and substitute into (C-2). Here, only asymptotic solutions up to $O\left(\varepsilon^{2}\right)$ are considered and we find

$$
\begin{aligned}
0=\left(\theta_{0}+\varepsilon \theta_{1}+\varepsilon^{2} \theta_{2}+\cdots\right)^{\prime \prime}+\left(\mathrm{Gr}_{0}+\varepsilon \mathrm{Gr}_{1}\right. & \left.+\varepsilon^{2} \mathrm{Gr}_{2}+\cdots\right) \\
& \times \exp \left(\theta_{0}+\varepsilon\left(\theta_{1}-\theta_{0}^{2}\right)+\varepsilon^{2}\left(\theta_{2}-2 \theta_{0} \theta_{1}+\theta_{0}^{3}\right)+\cdots\right) .
\end{aligned}
$$


We perturb Gr as well since the previously obtained numerical results suggest that the critical value of the Gruntfest number, $\mathrm{Gr}_{c}$, depends on $\mathrm{Ar}$, and hence on the small parameter $\varepsilon=\mathrm{Ar}^{-1} \ll 1$ (Figure 10).

After the exponential in the above expression is expanded as a Taylor series the zeroth-, first- and second-order equations of an asymptotic series are obtained:

$$
\begin{aligned}
\mathcal{O}(1): & \theta_{0}^{\prime \prime}+\mathrm{Gr}_{0} e^{\theta_{0}}=0, \\
\mathcal{O}(\varepsilon): & \theta_{1}^{\prime \prime}+\mathrm{Gr}_{0} e^{\theta_{0}} \theta_{1}=\mathrm{Gr}_{0} \theta_{0}^{2} e^{\theta_{0}}-\mathrm{Gr}_{1} e^{\theta_{0}}, \\
\mathcal{O}\left(\varepsilon^{2}\right): & \theta_{2}^{\prime \prime}+\mathrm{Gr}_{0} e^{\theta_{0}} \theta_{2}=\mathrm{Gr}_{0} e^{\theta_{0}}\left(2 \theta_{0} \theta_{1}-\theta_{0}^{3}-\frac{1}{2}\left(\theta_{1}-\theta_{0}^{2}\right)^{2}\right)-\mathrm{Gr}_{1} e^{\theta_{0}}\left(\theta_{1}-\theta_{0}^{2}\right)-\mathrm{Gr}_{2} e^{\theta_{0}} .
\end{aligned}
$$

Equation (C-4) has a known solution

$$
\theta_{0}(s)=\theta_{c}-2 \ln \cosh \left(\sqrt{\frac{\mathrm{Gr}_{0}}{2}} e^{\theta_{c} / 2} s\right)
$$

which satisfies the boundary conditions $\theta_{0}(0)=\theta_{c}$ as $\theta_{0}^{\prime}(0)=0$ [Fowler 1997]. The remaining boundary condition, $\theta_{0}(1)=0$ fixes the value of $\mathrm{Gr}_{0}$ and

$$
\mathrm{Gr}_{0}=2 \operatorname{acosh}^{2}\left[e^{\theta_{c} / 2}\right] e^{-\theta_{c}} .
$$

Maximising $\mathrm{Gr}_{0}$ with respect to $\theta_{c}$ yields the critical value of $\mathrm{Gr}$ at leading order which is $\mathrm{Gr}_{0, \mathrm{cr}} \approx 0.878$.

In order to find the first-order approximations we note that the homogeneous part of (C-5) is solved by $t_{\alpha}=\theta_{0}^{\prime}$ and $t_{\beta}=s \theta_{0}^{\prime}+2$. Based on the homogeneous solutions a particular solution $t_{p}$ can be constructed and we set $t_{p}=\xi t_{\alpha}+\psi t_{\beta}$. The parameters $\xi$ and $\psi$ are required to satisfy

$$
\begin{aligned}
0 & =\xi^{\prime} t_{\alpha}+\psi^{\prime} t_{\beta}, \\
\operatorname{Gr}_{0} \theta_{0}^{2} e^{\theta_{0}}-\mathrm{Gr}_{1} e^{\theta_{0}} & =\xi^{\prime} t_{\alpha}^{\prime}+\psi^{\prime} t_{\beta}^{\prime} .
\end{aligned}
$$

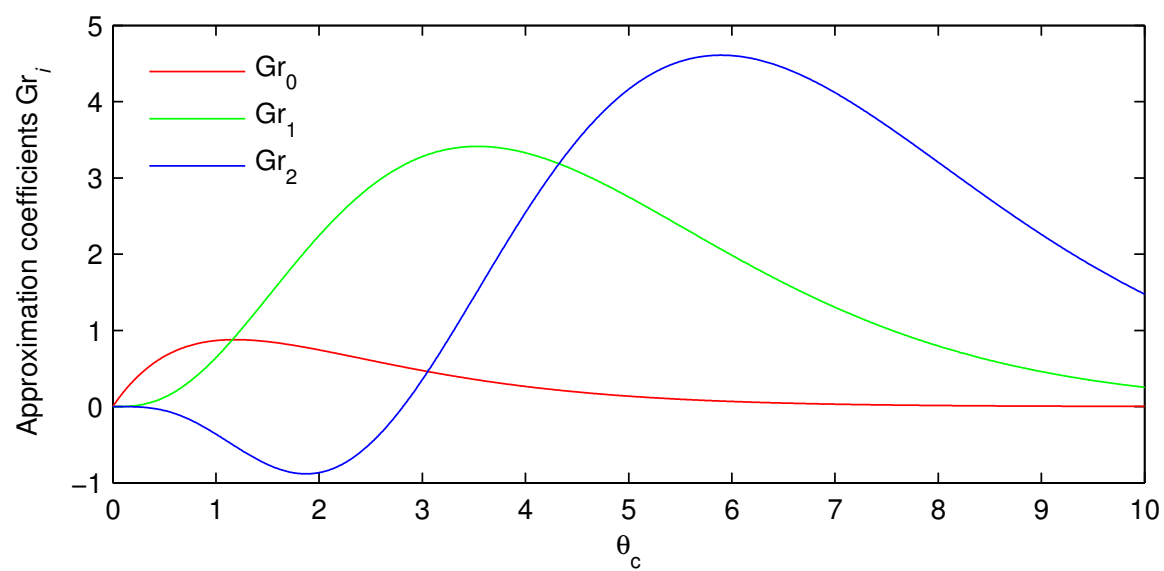

Figure 9. Approximation coefficients $\operatorname{Gr}_{i}(i=1,2,3)$ as a function of centre temperature $\theta_{c}$. 

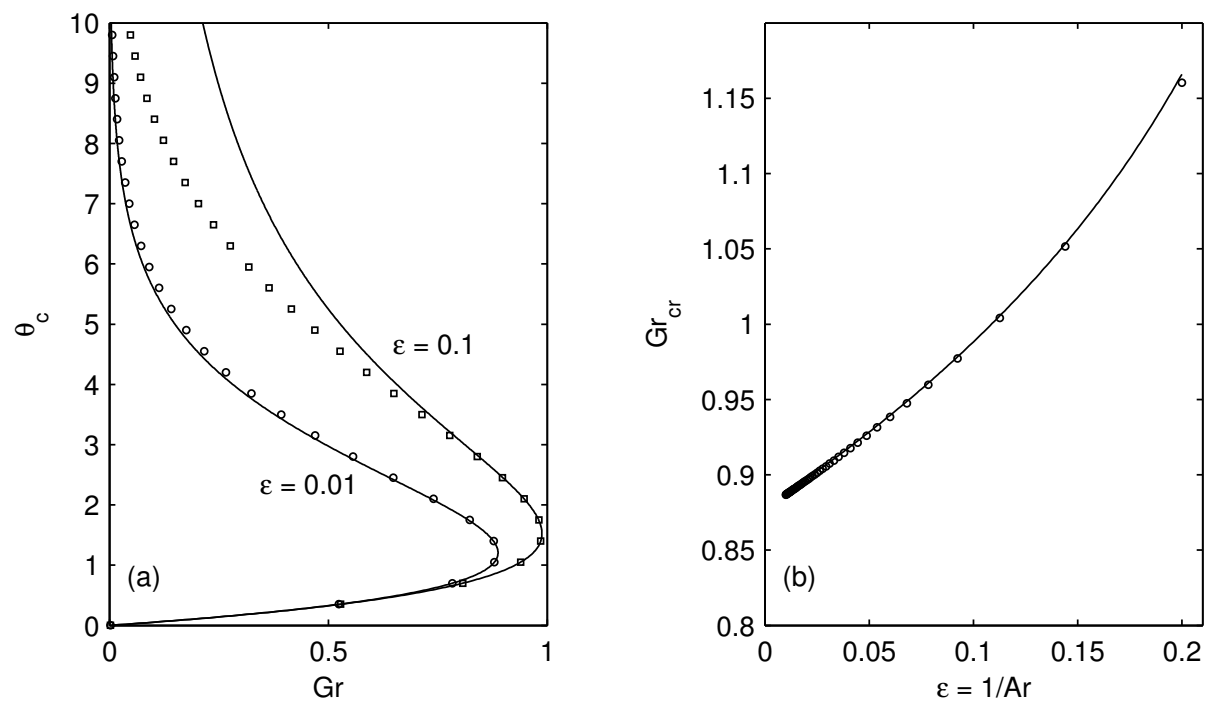

Figure 10. (a) Position of steady states of the heat equation (3-12) for $\varepsilon=0.1,0.01$. Squares and circles denote numerical results and the solid lines represent a three-term asymptotic approximation. The asymptotic approximation is suitable for $\theta_{c}<3$. (b) The critical Gruntfest number, $\mathrm{Gr}_{\mathrm{cr}}$, as a function of $\varepsilon$. Circles denote numerical results and the solid line represents a three-term asymptotic approximation. Note: here temperature is measured in units of Ar as described in the main text.

The even solution of $(\mathrm{C}-5)$ then reads

$$
\theta_{1}=\theta_{0}^{2}-2 \theta_{0}+4 s \theta_{0}^{\prime}+6-\frac{\mathrm{Gr}_{1}}{\mathrm{Gr}_{0}}-2 \theta_{0}^{\prime} \int \theta_{0} d s+C_{1}\left(s \theta_{0}^{\prime}+2\right),
$$

where $\mathrm{Gr}_{1}$ and $C_{1}$ are fixed by the boundary conditions $\theta_{1}(0)=\theta_{1}(1)=0$. After $\theta_{1}$ and $\mathrm{Gr}_{1}$ have been established the second order corrections $\mathrm{Gr}_{2}$ and $\theta_{2}$ can be computed analogously to the first-order correction.

In Figure 9 the coefficients of the expansion $\mathrm{Gr}=\mathrm{Gr}_{0}+\mathrm{Gr}_{1} \varepsilon+\mathrm{Gr}_{2} \varepsilon^{2}$ are shown as functions of the centre temperature $\theta_{c}$ and Figure 10(a) presents a comparison of the numerical results and asymptotic approximation. An approximation in the low temperature regime is capable of estimating the critical Gruntfest number to a good degree (see Figure 10(b)).

\section{References}

[Alevizos et al. 2014] S. Alevizos, T. Poulet, and E. Veveakis, "Thermo-poro-mechanics of chemically active creeping faults, 1: theory and steady state considerations", J. Geophys. Res.: Solid Earth 119:6 (2014), 4558-4582.

[Anand et al. 1987] L. Anand, K. H. Kim, and T. G. Shawki, "Onset of shear localization in viscoplastic solids", J. Mech. Phys. Solids 35:4 (1987), 407-429.

[Bai 1981] Y. L. Bai, "A criterion for thermo-plastic shear instability", pp. 277-284 in Shock waves and high-strain-rate phenomena in metals, edited by M. A. Myers and L. E. Murr, Plenum Press, New York, 1981.

[Bai 1982] Y. L. Bai, “Thermo-plastic instability in simple shear”, J. Mech. Phys. Solids 30:4 (1982), 195-207. 
[Balay et al. 2014] S. Balay, S. Abhyankar, M. F. Adams, J. Brown, P. Brune, K. Buschelman, V. Eijkhout, W. D. Gropp, D. Kaushik, M. G. Knepley, L. C. McInnes, K. Rupp, B. F. Smith, and H. Zhang, "PETSc Users Manual", software manual ANL-95/11, Argonne National Laboratory, 2014, http://www.mcs.anl.gov/petsc.

[Bauwens-Crowet et al. 1974] C. Bauwens-Crowet, J. M. Ots, and J. Bauwens, "The strain-rate and temperature dependence of yield of polycarbonate in tension, tensile creep and impact tests", J. Mater. Sci. 9 (1974), 1197-1201.

[Benallal and Bigoni 2004] A. Benallal and D. Bigoni, "Effects of temperature and thermo-mechanical couplings on material instabilities and strain localization of inelastic materials", J. Mech. Phys. Solids 52:3 (2004), 725-753.

[Benallal and Lemaitre 1991] A. Benallal and J. Lemaitre, "Creep in structures", pp. 223-235 in Creep in structures: Fourth IUTAM Symposium (Cracow, Poland, 1990), edited by M. Życzkowski, Springer, Berlin, 1991.

[Chaboche 1977] J. L. Chaboche, "Viscoplastic constitutive equations for the description of cyclic and anisotropic behavior of metals", B. Acad. Pol. Sci. Tech. 25:1 (1977), 33-42.

[Chaboche 2008] J. L. Chaboche, "A review of some plasticity and viscoplasticity constitutive theories", Int. J. Plast. 24:10 (2008), 1642-1693.

[Chan and Keller 1982] T. F. C. Chan and H. B. Keller, "Arc-length continuation and multigrid techniques for nonlinear elliptic eigenvalue problems”, SIAM J. Sci. Stat. Comput. 3:2 (1982), 173-194.

[Chen et al. 1989] H. T. Chen, A. S. Douglas, and R. Malek-Madani, "An asymptotic stability condition for inhomogeneous simple shear", Quart. Appl. Math. 47:2 (1989), 247-262.

[Cherukuri and Shawki 1995a] H. Cherukuri and T. Shawki, "An energy-based localization theory, II: effects of the diffusion, inertia, and dissipation numbers", Int. J. Plast. 11:1 (1995), 41-64.

[Cherukuri and Shawki 1995b] H. P. Cherukuri and T. G. Shawki, "An energy-based localization theory, I: basic framework", Int. J. Plast. 11:1 (1995), 15-40.

[Clifton 1980] R. J. Clifton, "Material response to ultra high loading rates", Report No. NMAB-356, NRC National Material Advisory Board (US) Report, 1980.

[Dodd and Bai 2012] B. Dodd and Y. Bai (editors), Adiabatic shear localization: frontiers and advances, 2nd ed., Elsevier, 2012.

[Fowler 1997] A. C. Fowler, Mathematical models in the applied sciences, Cambridge University Press, 1997.

[Freed and Walker 1993] A. D. Freed and K. P. Walker, "Viscoplasticity with creep and plasticity bounds", Int. J. Plast. 9:2 (1993), 213-242.

[Gaston et al. 2009] D. Gaston, C. Newman, G. Hansen, and D. Lebrun-Grandi, "MOOSE: A parallel computational framework for coupled systems of nonlinear equations", Nucl. Eng. Des. 239:10 (2009), 1768-1778.

[Gruntfest 1963] I. Gruntfest, "Thermal feedback in liquid flow: plane shear at constant stress", Trans. Soc. Rheol. 7 (1963), 195-207.

[Hill 1950] R. Hill, The mathematical theory of plasticity, Clarendon Press, Oxford, 1950.

[Hill 1962] R. Hill, “Acceleration waves in solids”, J. Mech. Phys. Solids 10 (1962), 1-16.

[Hückel and Baldi 1990] T. Hückel and G. Baldi, "Thermoplastic behavior of saturated clays: an experimental constitutive study”, J. Geotech. Eng. 116:12 (1990), 1778-1796.

[Hückel and Pellegrini 2002] T. Hückel and R. Pellegrini, "Reactive plasticity for clays: application to a natural analog of long-term geomechanical effects of nuclear waste disposal", Eng. Geology 64 (2002), 195-215.

[Johnson et al. 1964] W. Johnson, G. L. Baraya, and R. A. C. Slater, "On heat lines or lines of thermal discontinuity", Int. J. Mech. Sci. 6:6 (1964), 409-414.

[Khan et al. 2008] I. A. Khan, V. Bhasin, J. Chattopadhyay, and A. K. Ghosh, "On the equivalence of slip-line fields and work principles for rigid-plastic body in plane strain”, Int. J. Solids Struct. 45:25-26 (2008), 6416-6435.

[Kirk et al. 2006] B. S. Kirk, J. W. Peterson, R. H. Stogner, and G. F. Carey, "libMesh: A C++ library for parallel adaptive mesh refinement/coarsening simulations", Eng. Comput. 22:3-4 (2006), 237-254.

[Knoll and Keyes 2004] D. A. Knoll and D. E. Keyes, "Jacobian-free Newton-Krylov methods: a survey of approaches and applications”, J. Comput. Phys. 193:2 (2004), 357-397. 
[Law 2006] C. Law, Combustion physics, Cambridge University Press, 2006.

[Leroy and Molinari 1992] Y. Leroy and A. Molinari, "Stability of steady states in shear zones", J. Mech. Phys. Solids 40 (1992), 181-212.

[Lubliner 2008] J. Lubliner, Plasticity theory, Dover Publications, Mineola, NY, 2008.

[Needleman and Tvergaard 1992] A. Needleman and V. Tvergaard, "Analyses of plastic flow localization in metals", Appl. Mech. Rev. (ASME) 45:3S (1992), 1394557.

[Perzyna 1966] P. Perzyna, "Fundamental problems in viscoplasticity", Adv. Appl. Mech. 9 (1966), 243-377.

[Regenauer-Lieb et al. 2013a] K. Regenauer-Lieb, M. Veveakis, T. Poulet, F. Wellmann, A. Karrech, J. Liu, J. Hauser, C. Schrank, O. Gaede, and M. Trefry, "Multiscale coupling and multiphysics approaches in earth sciences: applications", $J$. Coupled Syst. Multiscale Dyn. 1:3 (2013), 281-323.

[Regenauer-Lieb et al. 2013b] K. Regenauer-Lieb, M. Veveakis, T. Poulet, F. Wellmann, A. Karrech, J. Liu, J. Hauser, C. Schrank, O. Gaede, and M. Trefry, "Multiscale coupling and multiphysics approaches in earth sciences: theory", J. Coupled Syst. Multiscale Dyn. 1:1 (2013), 49-73.

[Rice 1977] J. R. Rice, "The localization of plastic deformation", pp. 207-220 in 14th International Congress on Theoretical and Applied Mechanics (ICTAM) (Delft, Netherlands, 1976), edited by W. Koiter, North-Holland Publishing Company, Amsterdam, 1977.

[Rosakis et al. 2000] P. Rosakis, A. J. Rosakis, G. Ravichandran, and J. Hodowany, "A thermodynamic internal variable model for the partition of plastic work into heat and stored energy in metals", J. Mech. Phys. Solids 48:3 (2000), 581-607.

[Rudnicki and Rice 1975] J. W. Rudnicki and J. R. Rice, "Conditions for the localization of deformation in pressure sensitive dilatant materials", J. Mech. Phys. Solids 23 (1975), 371-394.

[Taylor and Quinney 1934] G. Taylor and H. Quinney, "The latent energy remaining in a metal after cold working", Proc. Royal Soc., Ser. A. 143 (1934), 307-326.

[Vardoulakis 2002] I. Vardoulakis, "Steady shear and thermal run-away in clayey gouges", Int. J. Solids Struct. 39 (2002), 3831-3844.

[Veveakis et al. 2007] E. Veveakis, I. Vardoulakis, and G. D. Toro, "Thermoporomechanics of creeping landslides: the 1963 Vaiont slide, northern Italy", J. Geophys. Res. 112 (2007), F03026.

[Veveakis et al. 2010] E. Veveakis, S. Alevizos, and I. Vardoulakis, "Chemical reaction capping of thermal instabilities during shear of frictional faults", J. Mech. Phys. Solids 58:9 (2010), 1175-1194.

[Veveakis et al. 2014] E. Veveakis, T. Poulet, and S. Alevizos, "Thermo-poro-mechanics of chemically active creeping faults, 2: transient considerations", J. Geophys. Res.: Solid Earth 119:6 (2014), 4583-4605.

Received 16 Apr 2015. Revised 19 Oct 2015. Accepted 24 Oct 2015.

MARTIN K. PAESOLD: martin.paesold@gmail.com

School of Mathematics and Statistics, University of Western Australia, 35 Stirling Hwy, Crawley WA 6009, Australia and

CSIRO Earth Science and Resource Engineering, 26 Dick Perry Avenue, Kensington, WA 6151, Australia

ANDREW P. BASSOM: andrew. bassom@utas.edu.au

School of Mathematics and Physics, University of Tasmania, Private Bag 37, Hobart TAS 7001, Australia

and

School of Mathematics and Statistics, University of Western Australia, 35 Stirling Hwy, Crawley, WA6009, Australia

KLAUS REgEnAUER-LIEB: regenau@gmail.com

School of Petroleum Engineering, University of New South Wales, Tyree Energy Technologies Building, Anzac Parade,

Kensington NSW 2052, Australia

e.veveakis@unsw.edu.au

MANOLIS VEVEAKIS: e.veveakis@unsw.edu.au 
School of Petroleum Engineering, University of New South Wales, Tyree Energy Technologies Building, Anzac Parade, Kensington NSW 2052, Australia

and

School of Mathematics and Statistics, University of Western Australia, 35 Stirling Hwy, Crawley, WA6009, Australia 


\title{
A SIMPLE HARD-PARTICLE COLLISION MODEL WITH A SMOOTH TRANSITION TO FULL SLIP
}

\author{
M. B. RUBIN
}

\begin{abstract}
Hard-particle models assume that the collision between rigid bodies is instantaneous and they propose algebraic equations for the jumps in the motions of the rigid bodies based on an assumed functional form for the impulsive force acting during the collision process. These hard-particle models have the advantage that they are significantly less computationally expensive than soft-particle models. Moreover, they are sufficiently accurate for some applications and can also be used for preliminary analysis of complicated processes with large numbers of particle interactions. Here, a simple three parameter hard-particle collision model is developed which predicts a smooth transition from normal collisions with no slip to oblique collisions with full slip. This model automatically satisfies a number of physical restrictions on the impulsive force. An additional physical restriction on nonnegative energy dissipation places nontrivial restrictions on the model parameters. Examples show the importance of improved modeling of the transition region from normal to oblique collisions with full slip.
\end{abstract}

\section{Introduction}

The mechanics of collision of two bodies is complicated by dynamic wave propagation, stick-slip frictional contact conditions and plastic dissipation [Goldsmith 1960]. The Distinct Element Method (DEM) is currently being used to model a broad range of physical phenomena like: granular flow [Walton 2004; Di Renzo and Di Maio 2004; Ketterhagen et al. 2005], particle fluids [Lu et al. 2005; Prasanth et al. 2012], studies of agglomeration [Kosinski and Hoffmann 2010; 2011], powder technology [Liu et al. 2011] and shock waves in jointed geological media [Morris et al. 2004]. Most often the DEM methods model the interaction of collections of rigid bodies with various shapes. The equations of motion of the rigid bodies are integrated using constitutive equations for the interactions between bodies that are in contact with each other. Soft-particle contact models use information about the virtual penetration regions of the bodies to determine the normal and shearing components of the contact force. The constitutive equations for the contact force attempt to model details of elastic and inelastic effects that are relevant for the specific application. In addition to the extensive computational effort to determine the contact regions, it is necessary to use small time steps to accurately integrate the equations of motion over the short interaction times associated with abrupt collision events.

The computational effort in DEM calculations to identify and analyze the virtual penetration regions can be significantly reduced when the bodies have simple shapes like spheres. Additional reduction in computational effort can be obtained by simplifying the contact events as instantaneous collisions. This simplification uses hard-particle models which assume that the contact region is small enough that only

Keywords: coefficient of restitution, collision, friction, hard-particle, rigid bodies, spheres. 
a single force acts at the point of contact between the bodies. Also, it is assumed that the collision events are separated in time so that at any instant only a single contact process operates between two bodies. Details of the contact event are modeled by proposing constitutive equations for the impulsive force that acts between the bodies at the point of contact. Then, the equations of motion of the rigid bodies are integrated to obtain algebraic equations that predict the motion of the bodies just after the collision in terms of the motion of the bodies just before the collision. Hard-particle models have the advantage that they are significantly less computationally expensive than soft-particle models. Moreover, they are sufficiently accurate for some applications and can also be used for preliminary analysis of complicated processes with large numbers of particle interactions.

A review of the numerical simulation of inelastic, frictional particle-particle interactions can be found in [Walton 1993]. More recent reviews discuss: discrete particle modeling of fluidized beds [Deen et al. 2007] the extension of normal force models [Kruggel-Emden et al. 2007]; the simulation of particulate systems [Zhu et al. 2007]; and particle collision modeling [Meyer and Deglon 2011]. Ketterhagen et al. [2005] studied flows with a range of solid fractions to examine the limits of hard-particle collision models, which are typically valid for dilute systems. Also, it is noted that Stevens and Hrenya [2005] have presented a comparison of predictions of soft-sphere models and measurements of normal impacts.

The algebraic equations that describe the jumps in the motion of two colliding rigid bodies have been discussed in [Rubin 1998; Chatterjee and Ruina 1998]. These equations are based on an energetic coefficient of restitution $\eta$ introduced by Ivanov [1992]. More specifically, the work in [Rubin 1998] discusses physical restrictions on constitutive equations for the impulsive force acting between the two rigid bodies during hard-particle collision events.

Walton [1993, sec. 25.5.1] discusses a hard-particle model for the collision of spheres that is based on the elastic, frictional solutions of the dynamic equations of elasticity described in [Maw et al. 1976; 1981]. This model depends on three parameters: the usual coefficient of restitution $e$, which connects jumps in the normal component of the relative velocity of the contact points of the bodies; a coefficient of rotational restitution $\beta$, which connects jumps in the tangential component of the relative velocity of the contact points of the bodies; and the constant shear coefficient $\mu_{s}$ which controls the ratio of the shear component of the impulsive force to its normal component in a full slip region. More specifically, the model is based on two straight lines in the space of auxiliary parameters $\left\{\psi_{1}, \psi_{2}\right\}$, which are measures of the obliquity of the collision event at the point of contact, just before and just after collision, respectively. One line characterizes oblique collisions with full slip and the other line characterizes partial slip during the transition from normal to oblique collisions with full slip. In particular, the model has a sharp transition at the intersection of these two lines.

The objective of the present paper is to analyze the model proposed in [Walton 1993] from the perspective of the physical restrictions discussed in [Rubin 1998]. This analysis reveals that the transition region from zero slip to full slip is large with respect to the angle of obliquity. Consequently, it is important to improve the modeling of this transition region from normal to oblique collisions with full slip. Moreover, the present work suggests that the rotational coefficient of restitution be replaced by a shear coefficient $\mu$, which characterizes the ratio of the shear component of the impulsive force to its normal component. This shear coefficient increases from zero for normal collisions to a maximum constant value $\mu_{s}$ for oblique collisions with full slip. Specifically, a three parameter model is proposed with a smooth transition from no slip to full slip. All three parameters can be determined directly from experimental data or from 

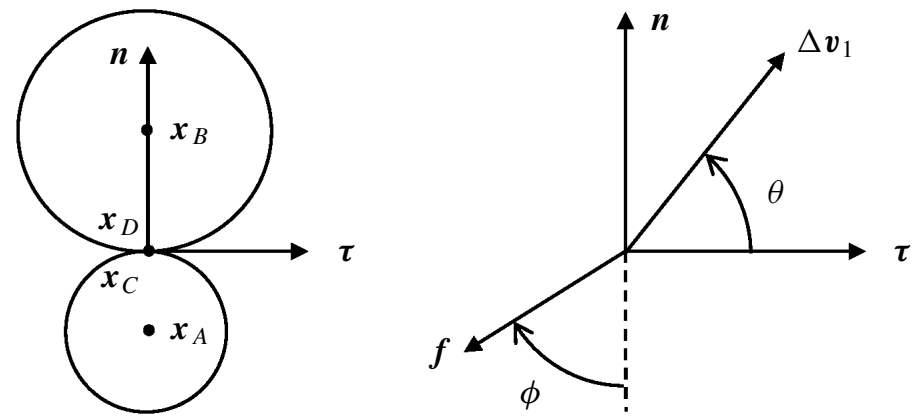

Figure 1. Sketch of the spheres, the unit outward normal vector $\boldsymbol{n}$ to sphere $(A)$, the unit tangent vector $\tau$ and the directions of $\Delta \boldsymbol{v}_{1}$ and $f$.

results of simulations using more complicated soft-particle models. In addition, a restriction is imposed on the model parameters which ensures non-negative energy dissipation in the collision event.

An outline of this paper is as follows. Section 2 reviews the basic equations of the formulation in [Rubin 1998] and specializes these equations to collision of two spheres. In particular, this section reviews physical restrictions that are placed on constitutive equations for the impulsive force acting during the collision event. Section 3 proposes a constitutive equation for the impulsive force that exhibits a smooth transition from normal to oblique collisions. Section 4 reviews the hard-particle model of Walton [1993] and Section 5 compares the smooth transition model with that of Walton. Section 6 considers the example of a sphere that bounces off a fixed horizontal plane and Section 7 presents conclusions.

\section{Basic equations}

In this section the general formulation presented in [Rubin 1998] is specialized to the case of collision of two rigid spheres. For simplicity, a number of equations are summarized without further reference to the work in [Rubin 1998]. Specifically, consider two spherical rigid bodies with masses $\left\{m_{A}, m_{B}\right\}$, radii $\left\{R_{A}, R_{B}\right\}$ and moments of inertia $\left\{I_{A}, I_{B}\right\}$ about their centers of mass, which have positions $\left\{\boldsymbol{x}_{A}, \boldsymbol{x}_{B}\right\}$ relative to a fixed origin (see Figure 1). Just before collision, the centers of mass have velocities $\left\{\boldsymbol{v}_{A 1}, \boldsymbol{v}_{B 1}\right\}$ and the bodies have angular velocities $\left\{\omega_{A 1}, \omega_{B 1}\right\}$. Just after collision, the centers of mass have velocities $\left\{\boldsymbol{v}_{A 2}, \boldsymbol{v}_{B 2}\right\}$ and the bodies have angular velocities $\left\{\boldsymbol{\omega}_{A 2}, \boldsymbol{\omega}_{B 2}\right\}$.

The collision event is considered to be instantaneous with only a point force acting between the bodies. Specifically, let $\hat{\boldsymbol{F}}$ denote the impulsive force applied by body $B$ on body $A$ at the common contact point which is denoted as $\boldsymbol{x}_{C}$ for the material point attached to body $A$ and by $\boldsymbol{x}_{D}$ for the material point attached to body $B$. Also, let $\boldsymbol{n}$ be the unit outward normal to the body $A$ at the impact point $\boldsymbol{x}_{C}=\boldsymbol{x}_{D}$, such that

$$
\boldsymbol{n}=\frac{\boldsymbol{x}_{B / A}}{\left|\boldsymbol{x}_{B / A}\right|}, \quad \boldsymbol{n} \cdot \boldsymbol{n}=1, \quad \boldsymbol{x}_{C / A}=R_{A} \boldsymbol{n}, \quad \boldsymbol{x}_{D / B}=-R_{B} \boldsymbol{n},
$$

where for simplicity use has been made of the notation

$$
x_{B / A}=x_{B}-x_{A} .
$$


The impulsive forms of the balances of linear and angular momentum (about the centers of mass) can be solved to deduce that

$$
\begin{array}{rlrl}
\boldsymbol{v}_{A 2} & =\boldsymbol{v}_{A 1}+\frac{1}{m_{A}} \hat{\boldsymbol{F}}, & \boldsymbol{v}_{B 2} & =\boldsymbol{v}_{B 1}-\frac{1}{m_{B}} \hat{\boldsymbol{F}}, \\
\boldsymbol{\omega}_{A 2} & =\boldsymbol{\omega}_{A 1}+\frac{R_{A}}{I_{A}} \boldsymbol{n} \times \hat{\boldsymbol{F}}, & \boldsymbol{\omega}_{B 2}=\boldsymbol{\omega}_{B 1}+\frac{R_{B}}{I_{B}} \boldsymbol{n} \times \hat{\boldsymbol{F}} .
\end{array}
$$

Moreover, the velocities $\left\{\boldsymbol{v}_{C 1}, \boldsymbol{v}_{D 1}\right\}$ of the contact material points just before the collision and the velocities $\left\{\boldsymbol{v}_{C 2}, \boldsymbol{v}_{D 2}\right\}$ just after the collision are given by

$$
\begin{array}{ll}
\boldsymbol{v}_{C 1}=\boldsymbol{v}_{A 1}+R_{A} \boldsymbol{\omega}_{A 1} \times \boldsymbol{n}, & \boldsymbol{v}_{D 1}=\boldsymbol{v}_{B 1}-R_{B} \boldsymbol{\omega}_{B 1} \times \boldsymbol{n}, \\
\boldsymbol{v}_{C 2}=\boldsymbol{v}_{A 2}+R_{A} \boldsymbol{\omega}_{A 2} \times \boldsymbol{n}, & \boldsymbol{v}_{D 2}=\boldsymbol{v}_{B 2}-R_{B} \boldsymbol{\omega}_{B 2} \times \boldsymbol{n} .
\end{array}
$$

Next, it is convenient to define the velocity differences of the contact points $\left\{\Delta \boldsymbol{v}_{1}, \Delta \boldsymbol{v}_{2}\right\}$ just before and just after the collision by the expressions

$$
\Delta \boldsymbol{v}_{1}=\boldsymbol{v}_{C 1}-\boldsymbol{v}_{D 1}, \quad \Delta \boldsymbol{v}_{2}=\boldsymbol{v}_{C 2}-\boldsymbol{v}_{D 2},
$$

and to recall that

$$
\Delta \boldsymbol{v}_{2}=\Delta \boldsymbol{v}_{1}+\frac{1}{m} \boldsymbol{A} \hat{\boldsymbol{F}}, \quad \boldsymbol{A}=\left(\frac{1+I_{m}}{I_{m}}\right) \boldsymbol{I}-\left(\frac{1}{I_{m}}\right) \boldsymbol{n} \otimes \boldsymbol{n},
$$

where $\boldsymbol{A}$ is a second order tensor and the effective mass $m$ for linear momentum and the parameter $I_{m}$ associated with angular momentum are defined by

$$
m=\frac{m_{A} m_{B}}{m_{A}+m_{B}}, \quad I_{m}=\frac{I_{A} I_{B}}{m\left(I_{A} R_{B}^{2}+I_{B} R_{A}^{2}\right)} .
$$

Also, $\boldsymbol{a} \otimes \boldsymbol{b}$ denotes the tensor product between two vectors $\{\boldsymbol{a}, \boldsymbol{b}\}$. Here, the spherical bodies are assumed to have spherically symmetric mass distributions but are allowed to have inhomogeneous mass distributions radially.

The incident slip velocity $\Delta \boldsymbol{v}_{1}$ and the unit normal $\boldsymbol{n}$ form a plane which contains the slip direction $\tau$, such that

$$
\Delta \boldsymbol{v}_{1}=\frac{\left|\Delta \boldsymbol{v}_{1}\right|}{\sqrt{1+\psi_{1}^{2}}}\left(\boldsymbol{n}+\psi_{1} \boldsymbol{\tau}\right), \quad \psi_{1}=\tan \theta \geq 0, \quad \boldsymbol{\tau}=\frac{\Delta \boldsymbol{v}_{1}-\left(\Delta \boldsymbol{v}_{1} \cdot \boldsymbol{n}\right) \boldsymbol{n}}{\left|\Delta \boldsymbol{v}_{1}-\left(\Delta \boldsymbol{v}_{1} \cdot \boldsymbol{n}\right) \boldsymbol{n}\right|}
$$

where $\theta$ is the incident angle of obliquity (see Figure 1). It follows that since

$$
\Delta \boldsymbol{v}_{1} \cdot \boldsymbol{n}>0
$$

sphere $A$ collides with sphere $B$.

Denoting $\left\{T_{1}, T_{2}\right\}$ as the kinetic energy of the two-particle system just before and just after the collision

$$
\begin{aligned}
& T_{1}=\frac{1}{2} m_{A} \boldsymbol{v}_{A 1} \cdot \boldsymbol{v}_{A 1}+\frac{1}{2} I_{A} \boldsymbol{\omega}_{A 1} \cdot \boldsymbol{\omega}_{A 1}+\frac{1}{2} m_{B} \boldsymbol{v}_{B 1} \cdot \boldsymbol{v}_{B 1}+\frac{1}{2} I_{B} \boldsymbol{\omega}_{B 1} \cdot \boldsymbol{\omega}_{B 1}, \\
& T_{2}=\frac{1}{2} m_{A} \boldsymbol{v}_{A 2} \cdot \boldsymbol{v}_{A 2}+\frac{1}{2} I_{A} \boldsymbol{\omega}_{A 2} \cdot \boldsymbol{\omega}_{A 2}+\frac{1}{2} m_{B} \boldsymbol{v}_{B 2} \cdot \boldsymbol{v}_{B 2}+\frac{1}{2} I_{B} \boldsymbol{\omega}_{B 2} \cdot \boldsymbol{\omega}_{B 2},
\end{aligned}
$$


it can be shown that

$$
T_{1}-T_{2}=-\hat{\boldsymbol{F}} \cdot \Delta \boldsymbol{v}_{1}-\frac{1}{2 m} \boldsymbol{A} \cdot(\hat{\boldsymbol{F}} \otimes \hat{\boldsymbol{F}}),
$$

where $\boldsymbol{A} \cdot(\boldsymbol{a} \otimes \boldsymbol{b})=\operatorname{tr}\left[\boldsymbol{A}(\boldsymbol{a} \otimes \boldsymbol{b})^{T}\right]$ is the inner product between the two second order tensors $\{\boldsymbol{A}, \boldsymbol{a} \otimes \boldsymbol{b}\}$.

In [Rubin 1998] $\hat{\boldsymbol{F}}$ was expressed in the form

$$
\hat{\boldsymbol{F}}=f \boldsymbol{f}, \quad \boldsymbol{f} \cdot \boldsymbol{f}=1, \quad f=m(1+\eta)\left[\frac{-\boldsymbol{f} \cdot \Delta \boldsymbol{v}_{1}}{\boldsymbol{A} \cdot(\boldsymbol{f} \otimes \boldsymbol{f})}\right]>0,
$$

which can be used to show that (2-11) reduces to

$$
T_{1}-T_{2}=\frac{1}{2} m\left|\Delta \boldsymbol{v}_{1}\right|^{2} \Delta T, \quad \Delta T=\frac{1}{2} m\left(1-\eta^{2}\right)\left[\frac{\left(\boldsymbol{f} \cdot \Delta \boldsymbol{v}_{1} /\left|\Delta \boldsymbol{v}_{1}\right|\right)^{2}}{\boldsymbol{A} \cdot(\boldsymbol{f} \otimes \boldsymbol{f})}\right] .
$$

In particular, the work in [Rubin 1998] suggests that the constitutive equation for the impulsive force must satisfy the following four physical restrictions.

(P1) The impulse is not orthogonal to the relative velocity of the contact points just before the collision

$$
\hat{\boldsymbol{F}} \cdot \Delta \boldsymbol{v}_{1} \neq 0
$$

(P2) The impulse has a component that resists penetration and aids separation of the two bodies

$$
\hat{\boldsymbol{F}} \cdot(-\boldsymbol{n})>0 .
$$

(P3) The two bodies have a tendency to separate after the collision event

$$
\Delta \boldsymbol{v}_{2} \cdot(-n) \geq 0
$$

(P4) The dissipation of kinetic energy during the collision event is non-negative

$$
T_{1}-T_{2} \geq 0
$$

\section{A constitutive equation for the impulsive force}

Once a constitutive equation for the impulsive force $\hat{\boldsymbol{F}}$ is specified the motion of the two particles just after collision is determined by the equations (2-3). This section presents a constitutive equation for $\hat{\boldsymbol{F}}$. First it is noted that with the help of (2-9) and (2-12), the restrictions (2-14) and (2-15) require

$$
-\boldsymbol{f} \cdot \Delta \boldsymbol{v}_{1}>0, \quad-\boldsymbol{f} \cdot \boldsymbol{n}>0 .
$$

Also, using (2-13), the restriction (2-17) on energy dissipation requires

$$
\begin{aligned}
& 1+\eta>0, \\
& 1-\eta \geq 0 .
\end{aligned}
$$

The work in [Maw et al. 1976; 1981] and [Walton 1993, Sec. 25.5.1] suggests that a model for hardparticle collision between two spheres can be formulated in terms of $\psi_{1}$ in (2-8) and $\psi_{2}$ defined by

$$
\psi_{1}=\frac{\Delta \boldsymbol{v}_{1} \cdot \boldsymbol{\tau}}{\Delta \boldsymbol{v}_{1} \cdot \boldsymbol{n}} \geq 0, \quad \psi_{2}=-\left[\frac{\Delta \boldsymbol{v}_{2} \cdot \boldsymbol{\tau}}{\Delta \boldsymbol{v}_{2} \cdot \boldsymbol{n}}\right]
$$


The parameters $\left\{\psi_{1}, \psi_{2}\right\}$ are measures of the obliquity of the collision event, just before and just after the collision, respectively. Moreover, the model in [Walton 1993] uses the standard coefficient of restitution $e$, which connects the normal components of relative velocities by

$$
\Delta \boldsymbol{v}_{2} \cdot \boldsymbol{n}=-e\left(\Delta \boldsymbol{v}_{1} \cdot \boldsymbol{n}\right), \quad 0 \leq e \leq 1
$$

which with the help of (2-9) ensures that the restriction (2-16) is satisfied. Here, use is made of (3-4) to obtain a constitutive equation for $\eta$. Specifically, from (2-6) and (2-12) it can be shown that (3-4) yields the constitutive equation

$$
(1+\eta)=(1+e)\left[\frac{\boldsymbol{A} \cdot(\boldsymbol{f} \otimes \boldsymbol{f})}{\boldsymbol{A} \cdot(-\boldsymbol{f} \otimes \boldsymbol{n})}\right]\left(\frac{\boldsymbol{n} \cdot \Delta \boldsymbol{v}_{1}}{-\boldsymbol{f} \cdot \Delta \boldsymbol{v}_{1}}\right) .
$$

The hard-particle model proposed by Walton [1993, Sec. 25.5.1] introduced a rotational coefficient of restitution $\beta$ associated with the tangential direction $\tau$ defined by

$$
\Delta \boldsymbol{v}_{2} \cdot \boldsymbol{\tau}=-\beta\left(\Delta \boldsymbol{v}_{1} \cdot \boldsymbol{\tau}\right), \quad-1 \leq \beta \leq 1
$$

Here, this constitutive assumption is replaced by a constitutive equation for a shear coefficient $\mu$, which is the ratio of the shearing component of the impulsive force to its normal component. Specifically, it is assumed that the impulsive force is in the $\boldsymbol{n}-\boldsymbol{\tau}$ plane with

$$
\boldsymbol{f}=-\frac{1}{\sqrt{1+\mu^{2}}}(\boldsymbol{n}+\mu \boldsymbol{\tau}), \quad \mu=\tan \phi>0,
$$

where $\phi$ is the friction angle (see Figure 1). Then, using (2-8) it can be shown that the restrictions (3-1) are automatically satisfied

$$
-\boldsymbol{f} \cdot \Delta \boldsymbol{v}_{1}=\frac{\left|\Delta \boldsymbol{v}_{1}\right|}{\sqrt{1+\psi_{1}^{2}} \sqrt{1+\mu^{2}}}\left(1+\mu \psi_{1}\right)>0, \quad-\boldsymbol{f} \cdot \boldsymbol{n}=\frac{1}{\sqrt{1+\mu^{2}}}>0 .
$$

It should be noted that although the impulsive force has been assumed to limited to the $\boldsymbol{n}-\boldsymbol{\tau}$ plane this does not mean that the motion of the spheres is two-dimensional since the angular velocities of the spheres need not be normal to this collision plane. Next, using (2-6), (2-8) and (3-7) it can be shown that

$$
1+\eta=\left(\frac{1+e}{1+\mu \psi_{1}}\right)\left[1+\left(\frac{1+I_{m}}{I_{m}}\right) \mu^{2}\right]>0,
$$

which shows that the restriction (3-2a) is automatically satisfied. Then, the restriction (3-2b) can be rewritten in the form

$$
1-\eta=2-\left(\frac{1}{1+\mu \psi_{1}}\right)\left[1+\left(\frac{1+I_{m}}{I_{m}}\right) \mu^{2}\right] \geq 0,
$$

which imposes a non-trivial restriction on the functional form for the shear coefficient $\mu$.

In order to motivate a functional form for $\mu$ use is made of (2-6), (2-8), (2-12), (3-3) (3-7), (3-8) and (3-9) to deduce that 


$$
\begin{aligned}
\hat{\boldsymbol{F}} & =-m(1+e) \frac{\left|\Delta \boldsymbol{v}_{1}\right|}{\sqrt{1+\psi_{1}^{2}}}(\boldsymbol{n}+\mu \boldsymbol{\tau}), \\
\Delta \boldsymbol{v}_{2} & =\frac{e\left|\Delta \boldsymbol{v}_{1}\right|}{\sqrt{1+\psi_{1}^{2}}}\left(-\boldsymbol{n}+\psi_{2} \boldsymbol{\tau}\right) \\
\psi_{2} & =\frac{1}{e}\left[\psi_{1}-(1+e)\left(\frac{1+I_{m}}{I_{m}}\right) \mu\right] .
\end{aligned}
$$

The hard-particle model in [Walton 1993] assumes that $\psi_{2}$ is a bi-linear function of $\psi_{1}$ with a negative slope in the region of partial slip and a positive slope in the region of full slip. Motivated by this model, a constitutive equation for $\mu$ is specified by the form

$$
\mu=\mu_{s} g(z), \quad g(z)=1-\langle 1-z\rangle^{1+\alpha}, \quad \alpha>0, \quad z=\frac{\psi_{1}}{\psi_{s}}, \quad \psi_{s}=(1+e)\left(\frac{1+I_{m}}{I_{m}}\right) \mu_{s},
$$

where $\langle x\rangle=\max (x, 0)$ denotes the Macaulay brackets. Thus, $\psi_{2}$ in (3-11) can be simplified to obtain

$$
\psi_{2}=\psi_{2}\left(\psi_{1}\right)=\frac{\psi_{s}}{e}[z-g(z)]
$$

It follows that

$$
\psi_{2}(0)=0, \quad \frac{d \psi_{2}}{d \psi_{1}}(0)=-\frac{\alpha}{e}, \quad \psi_{2}\left(\psi_{s}\right)=0, \quad \frac{d \psi_{2}}{d \psi_{1}}\left(\psi_{s}\right)=\frac{1}{e} .
$$

This means that the measure $\psi_{2}$ of the obliquity angle just after the collision is a smooth function of the measure $\psi_{1}$ of the obliquity angle just before the collision. The response characterizes partial slip with negative values of $\psi_{2}$ for $\psi_{1}<\psi_{s}$ and full slip with positive values of $\psi_{2}$ for $\psi_{1}>\psi_{s}$. Moreover, the transition to full slip occurs when $\psi_{2}$ changes sign with $\psi_{1}=\psi_{s}$. Also, the shear coefficient $\mu$ attains and maintains its maximum constant value $\mu_{s}$ during full slip.

This hard-particle model is characterized by three model parameters

$$
\left\{e, \mu_{s}, \alpha\right\}
$$

with $\alpha$ controlling the shape of the transition region from partial slip to full slip. The model will satisfy the physical restrictions (2-14)-(2-17) on the impulsive force when the values of these parameters ensure that (3-10) is satisfied for all collision events. In the following sections this model is compared with the hard-particle model of Walton [1993] and examples are considered which demonstrate the importance of the smooth transition in the partial slip region.

\section{The hard-particle collision model of Walton [1993]}

The hard-particle model proposed by Walton [1993, Sec. 25.5.1] is characterized by the usual coefficient of restitution $e$ in (3-4) and a rotational coefficient of restitution $\beta$ associated with the tangential direction $\boldsymbol{\tau}$ defined in (3-6) (see Figure 2). These expressions tacitly assume that $\Delta \boldsymbol{v}_{2}$ remains in the plane of $\left\{n, \Delta \boldsymbol{v}_{1}\right\}$ with

$$
\Delta \boldsymbol{v}_{2}=-\left[e\left(\Delta \boldsymbol{v}_{1} \cdot \boldsymbol{n}\right) \boldsymbol{n}+\beta\left(\Delta \boldsymbol{v}_{1} \cdot \boldsymbol{\tau}\right) \boldsymbol{\tau}\right],
$$


which with the help of (2-8) and (3-3) can be rewritten in the form

$$
\Delta \boldsymbol{v}_{2}=\frac{e\left|\Delta \boldsymbol{v}_{1}\right|}{\sqrt{1+\psi_{1}^{2}}}\left(-\boldsymbol{n}+\psi_{2} \boldsymbol{\tau}\right), \quad \psi_{2}=-\frac{\beta}{e} \psi_{1} .
$$

Moreover, Walton [1993] proposed a functional form for $\beta$ defined by

$$
\beta=\min \left(\beta_{0}, \beta^{*}\right), \quad \beta^{*}=-1+\frac{\psi_{s}}{\psi_{1}}, \quad 0<\beta_{0} \leq 1,
$$

where $\psi_{s}$ is given by (3-12) and it is noted that $K$ in [Walton 1993] is equal to $I_{m}$ here. It then follows that (4-2) and (4-3) yield

$$
\psi_{2}=-\frac{\beta_{0}}{e} \psi_{1} \text { for } 0 \leq \psi_{1} \leq \frac{\psi_{s}}{1+\beta_{0}}, \quad \psi_{2}=\frac{1}{e}\left(\psi_{1}-\psi_{s}\right) \text { for } \psi_{1} \geq \frac{\psi_{s}}{1+\beta_{0}},
$$

which satisfy the conditions (3-14) when $\beta_{0}=\alpha$. Moreover, using (3-12) the expression (3-11) can be rewritten in the form

$$
\psi_{2}=\frac{1}{e}\left[\psi_{1}-\psi_{s}\left(\frac{\mu}{\mu_{s}}\right)\right]
$$

Thus, comparing (4-2) and (4-5), replacing $\left\{\mu, \beta_{0}\right\}$ by $\left\{\mu_{W}, \alpha\right\}$ and using the definition of $z$ in (3-12) yields the constitutive equation

$$
\mu=\mu_{W}=(1+\beta) \mu_{s} z, \quad \beta=\min \left(\alpha, \beta^{*}\right), \quad \beta^{*}=-1+\frac{\psi_{s}}{\psi_{1}},
$$

which causes the model described in Sections 2 and 3 to be the same as Walton's hard-particle model. The associated value $\eta_{W}$ of $\eta$ is given by (3-9) with $\mu$ replaced by $\mu_{W}$

$$
\eta=\eta_{W}=1-\left(\frac{1}{1+\mu_{W} \psi_{1}}\right)\left[1+\left(\frac{1+I_{m}}{I_{m}}\right) \mu_{W}^{2}\right] .
$$

This functional form automatically satisfies the restriction

$$
1+\eta_{W}>0
$$

and must satisfy the restriction

$$
1-\eta_{W} \geq 0
$$

in order to ensure non-negative energy dissipation during the collision.

In summary, Walton's hard-particle model is given by (4-5)-(4-9) which depend on the same three parameters (3-15) as the smooth transition model proposed in Section 3.

\section{Comparison of the smooth model with Walton's model}

In the remainder of the paper attention is limited to the case of uniform spheres for which

$$
I_{A}=\frac{2}{5} m_{A} R_{A}^{2}, \quad I_{B}=\frac{2}{5} m_{B} R_{B}^{2}, \quad I_{m}=\frac{2}{5} .
$$




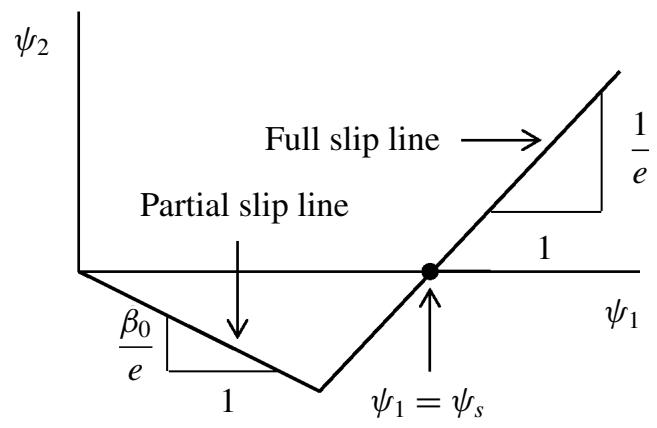

Figure 2. Sketch of the hard-sphere collision model of [Walton 1993].

The results in Figures 3-5 compare the response of the smooth model (denoted by $S$ ) with that of Walton's model (denoted by $W$ ) for

$$
\mu_{s}=0.3, \quad e=1 \text { or } 0.8, \quad \alpha=0.35,1 \text { or } 5 .
$$

where the value of $\alpha=0.35$ was suggested in [Walton 1993]. In these figures the subscript has been removed from $\mu_{W}$. These figures show that the differences between the two models can be significant, especially for the larger values of $\alpha$ when $e=0.8$. In particular, it can be seen from Figure 3(c) that for the smooth model the transition to full slip occurs at an obliquity angle $\theta=64.5^{\circ}$ so the transition region from no slip to full slip is large. Also, it can be seen from parts (d) and (e) of Figure 4 that during this transition region Walton's model predicts no dissipation whereas the smooth model predicts dissipation
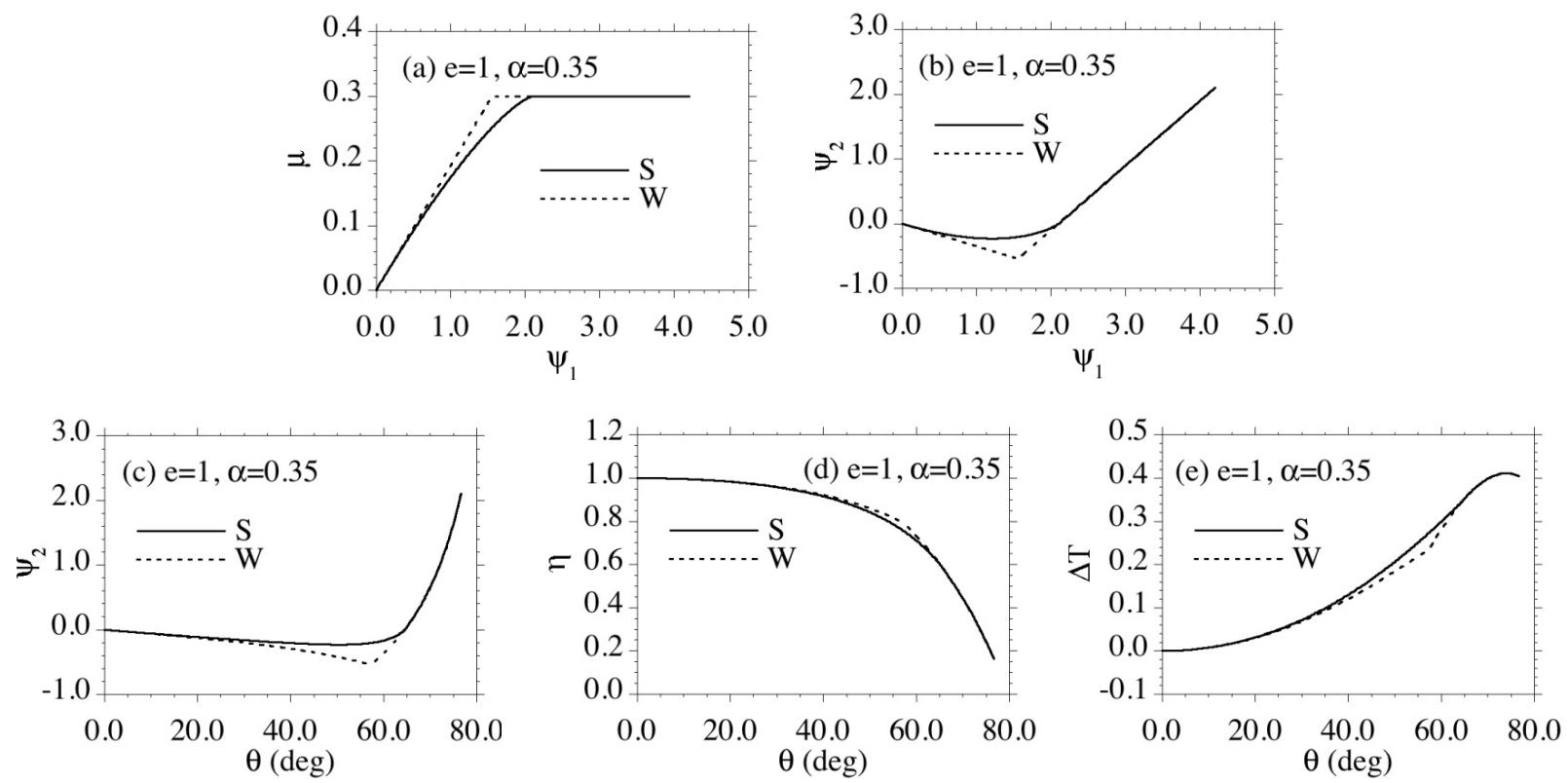

Figure 3. Comparison of the smooth model $(S)$ with Walton's model $(W)$ for $e=1$, $\alpha=0.35$ and $\mu_{s}=0.3$. 

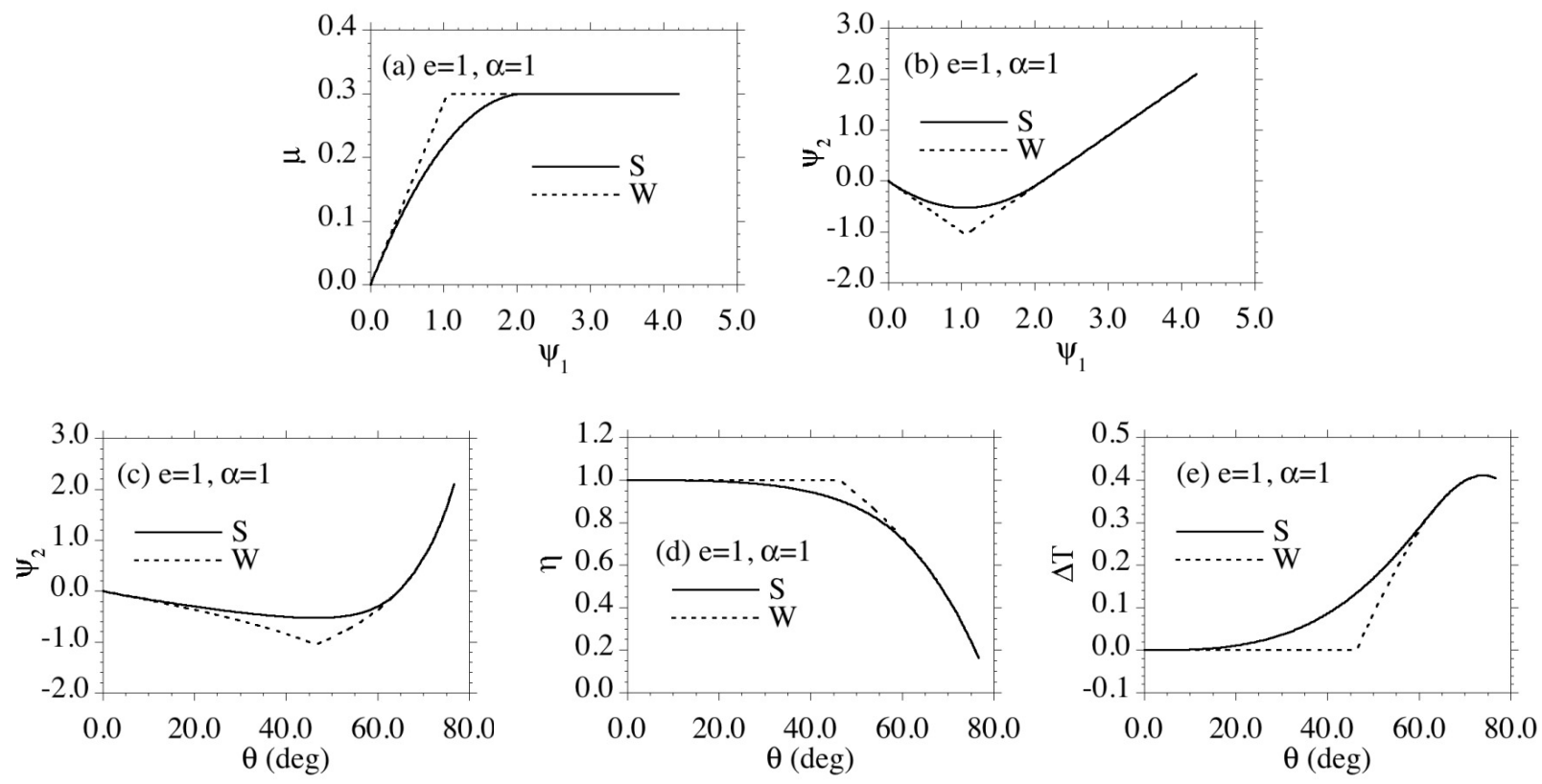

Figure 4. Comparison of the smooth model $(S)$ with Walton's model $(W)$ for $e=1$, $\alpha=1$ and $\mu_{s}=0.3$.
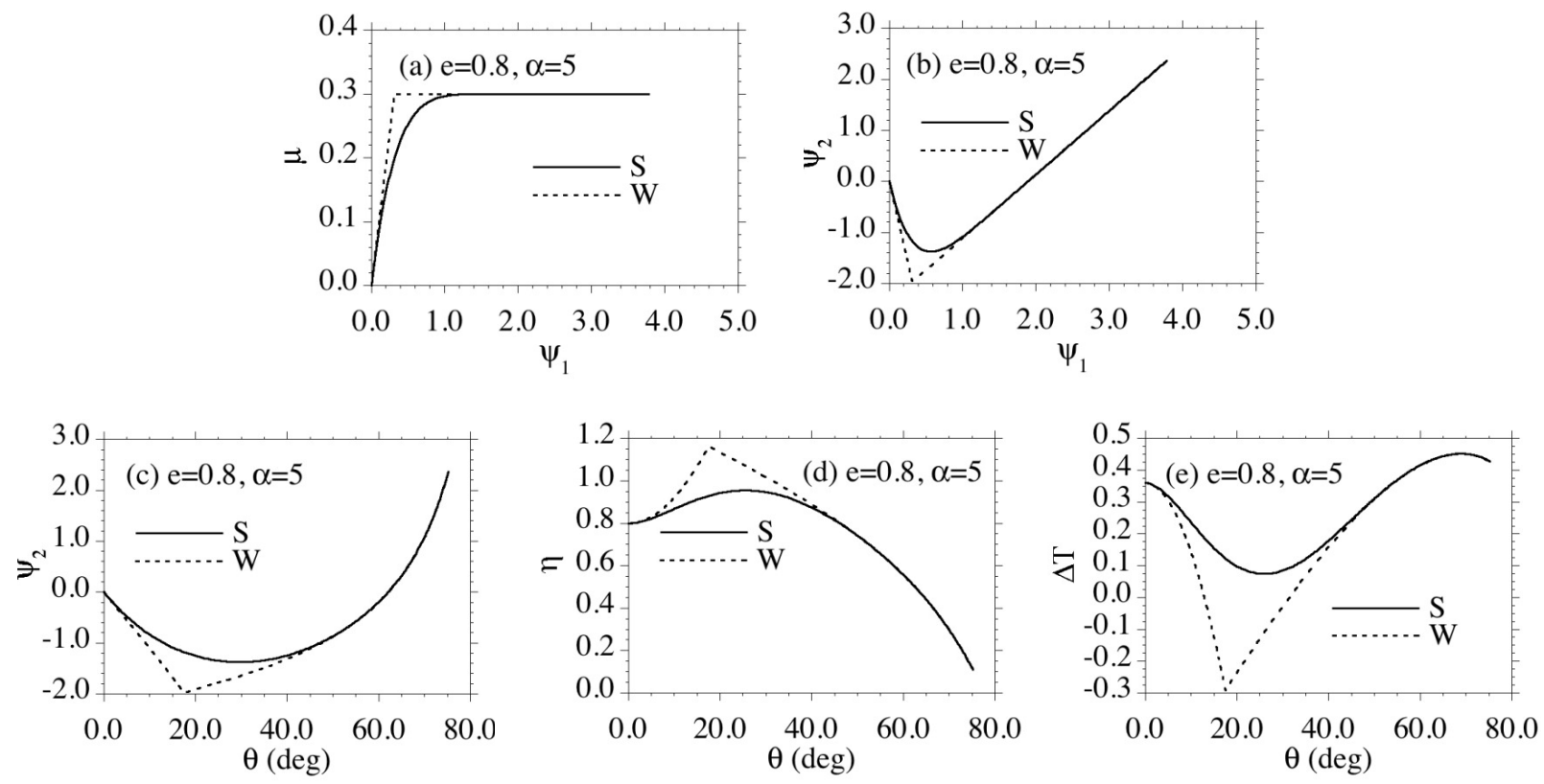

Figure 5. Comparison of the smooth model $(S)$ with Walton's model $(W)$ for $e=0.8$, $\alpha=5$ and $\mu_{s}=0.3$. 
over the entire range of the response. The results in Figures 3 and 4 satisfy the dissipation inequalities (3-9) and (3-10). Moreover, the parameters used for Figure 5 show that these inequalities are not trivially satisfied. In particular, it can be seen from parts (d) and (e) of Figure 5 that Walton's model would violate the dissipation inequality (3-10), while the smooth model would satisfy the dissipation inequality (3-10).

\section{Bouncing of a sphere off a fixed horizontal plane}

Figure 6 shows a sketch of a uniform sphere $(A)$ colliding with a fixed horizontal plane with attention limited to the vertical $\boldsymbol{e}_{1}-\boldsymbol{e}_{2}$ plane. Also, the motion of each sphere is simplified to be two-dimensional with its angular velocity in the $\boldsymbol{e}_{3}$ direction. The body force of gravity $b$ per unit mass acts in the positive $\boldsymbol{e}_{1}$ direction. The effect of gravity is used to determine the motion of the sphere between collision events but is neglected during the collisions. This approximation causes the model to limited to strong collision events.

Using the formulation in the previous sections the wall can be modeled by the sphere $(B)$ with the specifications

$$
m_{B} \gg m_{A}, \quad R_{B} \gg R_{A}, \quad \boldsymbol{v}_{B 1}=\boldsymbol{v}_{B 2}=\boldsymbol{v}_{D 1}=\boldsymbol{v}_{D 2}=0, \quad \boldsymbol{\omega}_{B 1}=\omega_{B 2}=0 .
$$

Next, consider a general collision event which occurs at $t=t_{n}$ with

$$
\begin{aligned}
\boldsymbol{v}_{A 1}\left(t_{n}\right) & =u_{1}\left(t_{n}\right) \boldsymbol{e}_{1}+w_{1}\left(t_{n}\right) \boldsymbol{e}_{2}, \quad u_{1}\left(t_{n}\right)>0, \quad \boldsymbol{\omega}_{A 1}\left(t_{n}\right)=\omega_{1}\left(t_{n}\right) \boldsymbol{e}_{3}, \\
\boldsymbol{v}_{A 2}\left(t_{n}\right) & =u_{2}\left(t_{n}\right) \boldsymbol{e}_{1}+w_{2}\left(t_{n}\right) \boldsymbol{e}_{2}, \quad u_{2}\left(t_{n}\right) \leq 0, \quad \boldsymbol{\omega}_{A 2}\left(t_{n}\right)=\omega_{2}\left(t_{n}\right) \boldsymbol{e}_{3}, \\
\Delta \boldsymbol{v}_{1}\left(t_{n}\right) & =\boldsymbol{v}_{C 1}\left(t_{n}\right)=u_{1}\left(t_{n}\right) \boldsymbol{e}_{1}+\left[w_{1}\left(t_{n}\right)+R_{A} \omega_{1}\left(t_{n}\right)\right] \boldsymbol{e}_{2}, \\
\Delta \boldsymbol{v}_{2}\left(t_{n}\right) & =\boldsymbol{v}_{C 2}\left(t_{n}\right)=u_{2}\left(t_{n}\right) \boldsymbol{e}_{1}+\left[w_{2}\left(t_{n}\right)+R_{A} \omega_{2}\left(t_{n}\right)\right] \boldsymbol{e}_{2}, \\
\boldsymbol{n} & =\boldsymbol{e}_{1}, \quad \boldsymbol{\tau}\left(t_{n}\right)=\frac{s_{1}\left(t_{n}\right)}{\left|s_{1}\left(t_{n}\right)\right|} \boldsymbol{e}_{2}, \quad s_{1}\left(t_{n}\right)=w_{1}\left(t_{n}\right)+R_{A} \omega_{1}\left(t_{n}\right),
\end{aligned}
$$
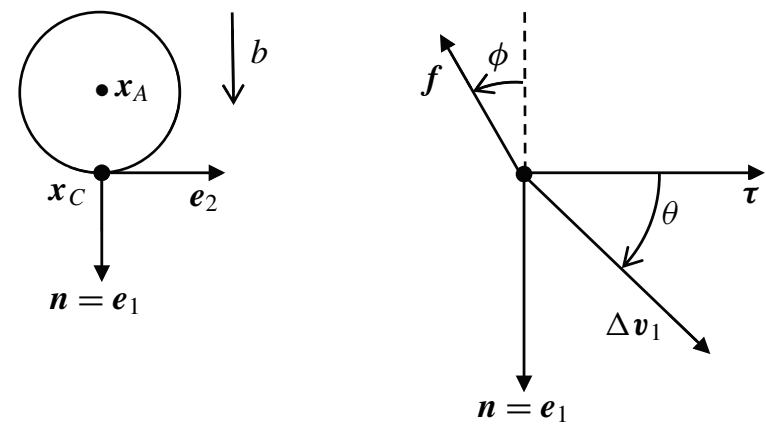

Figure 6. Sketch of a sphere colliding with a fixed horizontal plane showing the normal $\boldsymbol{n}$ and tangent $\boldsymbol{\tau}$ unit vectors and the directions of $\Delta \boldsymbol{v}_{1}$ and $\boldsymbol{f}$. 
where $s_{1}\left(t_{n}\right)$ is the slip velocity just before collision. Then, with the help of (2-3), (2-6)-(2-8), (2-13), (3-3), (3-7)-(3-9), (3-11)-(3-13), (5-1), (6-1) and (6-2) it follows that

$$
\begin{aligned}
& m=m_{A}, \quad \psi_{s}=\frac{7}{2}(1+e) \mu_{s}, \quad \eta_{n}=-1+\left[\frac{1+e+\mu_{s} \psi_{s} g_{n}^{2}}{1+\mu_{s} \psi_{s} z_{n} g_{n}}\right], \\
& \psi_{1}\left(t_{n}\right)=\frac{\left|s_{1}\left(t_{n}\right)\right|}{u_{1}\left(t_{n}\right)}, \quad z_{n}=\frac{\psi_{1}\left(t_{n}\right)}{\psi_{s}}, \quad g_{n}=1-\left\langle 1-z_{n}\right\rangle^{\alpha+1}, \\
& \psi_{2}\left(t_{n}\right)=-\frac{s_{1}\left(t_{n}\right)\left[w_{2}\left(t_{n}\right)+R_{A} \omega_{2}\left(t_{n}\right)\right]}{\left|s_{1}\left(t_{n}\right)\right| u_{2}\left(t_{n}\right)}, \quad \hat{\boldsymbol{F}}_{n}=-m_{A}(1+e) u_{1}\left(t_{n}\right)\left[\boldsymbol{e}_{1}+\mu_{s} g_{n} \frac{s_{1}\left(t_{n}\right)}{\left|s_{1}\left(t_{n}\right)\right|} \boldsymbol{e}_{2}\right], \\
& u_{2}\left(t_{n}\right)=-e u_{1}\left(t_{n}\right), \quad w_{2}\left(t_{n}\right)=w_{1}\left(t_{n}\right)-\frac{2}{7} u_{1}\left(t_{n}\right) \psi_{s} g_{n}\left[\frac{s_{1}\left(t_{n}\right)}{\left|s_{1}\left(t_{n}\right)\right|}\right], \\
& R_{A} \omega_{2}\left(t_{n}\right)=R_{A} \omega_{1}\left(t_{n}\right)-\frac{5}{7} u_{1}\left(t_{n}\right) \psi_{s} g_{n}\left[\frac{s_{1}\left(t_{n}\right)}{\left|s_{1}\left(t_{n}\right)\right|}\right] .
\end{aligned}
$$

Also, with the help of (5-1) the kinetic energy $T\left(t_{n}\right)$ just before the collision is given by

$$
T\left(t_{n}\right)=\frac{1}{2} m_{A}\left[u_{1}^{2}\left(t_{n}\right)+w_{1}^{2}\left(t_{n}\right)+\frac{2}{5} R_{A}^{2} \omega_{1}^{2}\left(t_{n}\right)\right] .
$$

Next, expressing the center of mass $\boldsymbol{x}_{A}$ and the angular velocity $\boldsymbol{\omega}_{A}$ of the sphere in the forms

$$
\boldsymbol{x}_{A}=x \boldsymbol{e}_{1}+y \boldsymbol{e}_{2}, \quad \boldsymbol{\omega}_{A}=\omega \boldsymbol{e}_{3},
$$

the balance laws of linear and angular momentum of the sphere between collisions require

$$
\ddot{x}=b, \quad \ddot{y}=0, \quad \dot{\omega}=0 .
$$

Using the values $\left\{u_{1}\left(t_{n}\right), w_{1}\left(t_{n}\right), \omega_{1}\left(t_{n}\right)\right\}$ just before the collision at $t=t_{n}$, the equations (6-3) determine the initial conditions $\left\{u_{2}\left(t_{n}\right), w_{2}\left(t_{n}\right), \omega_{2}\left(t_{n}\right)\right\}$ just after collision, with the initial values of $\{x, y, \omega\}$ for (6-6) given by

$$
x\left(t_{n}\right)=0, \quad \dot{x}\left(t_{n}\right)=u_{2}\left(t_{n}\right), \quad y\left(t_{n}\right)=y\left(t_{n}^{-}\right), \quad \dot{y}\left(t_{n}\right)=w_{2}\left(t_{n}\right), \quad \omega\left(t_{n}\right)=\omega_{2}\left(t_{n}\right),
$$

where $y\left(t_{n}^{-}\right)$is the value of $y$ just before the collision. Then, the solution of (6-6) is given by

$$
\begin{aligned}
& x(t)=u_{2}\left(t_{n}\right)\left(t-t_{n}\right)+\frac{1}{2} b\left(t-t_{n}\right)^{2}, \\
& y(t)=y\left(t_{n}\right)+w_{2}\left(t_{n}\right)\left(t-t_{n}\right), \\
& \omega(t)=\omega_{2}\left(t_{n}\right) \text { for } t_{n} \leq t \leq t_{n+1} .
\end{aligned}
$$

The value $t=t_{n+1}$ is the time when the next collision occurs with the wall

$$
x\left(t_{n+1}\right)=0 \Rightarrow t_{n+1}=t_{n}-\frac{2 u_{2}\left(t_{n}\right)}{b},
$$

with the value $y\left(t_{n+1}\right)$ for the collision at $t=t_{n+1}$ being specified by

$$
y\left(t_{n+1}\right)=y\left(t_{n}\right)-\frac{2 u_{2}\left(t_{n}\right) w_{2}\left(t_{n}\right)}{b} .
$$



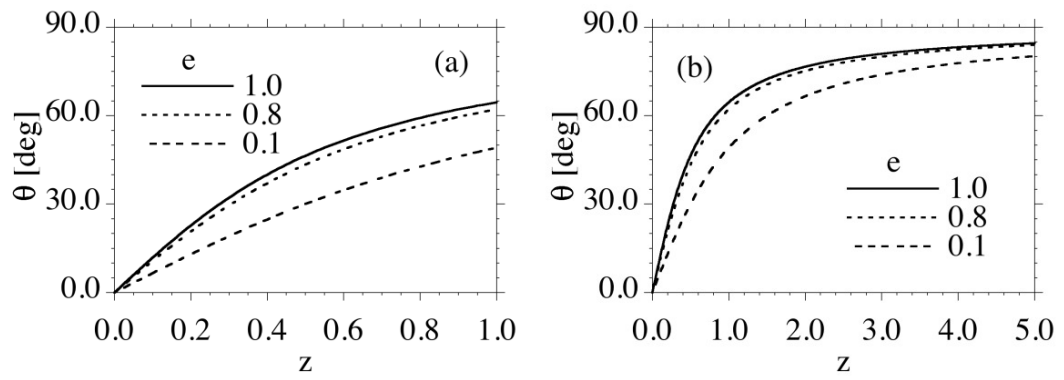

Figure 7. Values of the obliquity angle $\theta$ as a function of the normalized variable $z$.
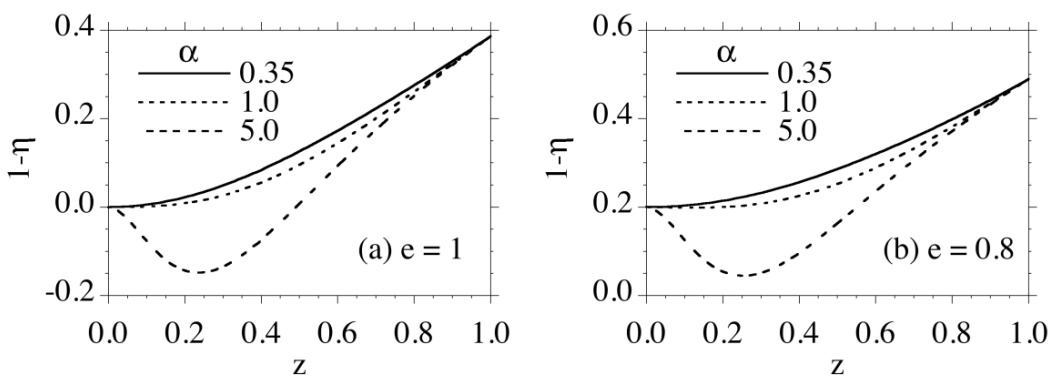

Figure 8. Examination of the restriction $1-\eta>0$ for positive energy dissipation on the parameters $e$ and $\alpha$.

Moreover, the values of $\left\{u_{1}\left(t_{n+1}\right), w_{1}\left(t_{n+1}\right), \omega_{1}\left(t_{n+1}\right)\right\}$ just before the collision at $t=t_{n+1}$ are given by

$$
u_{1}\left(t_{n+1}\right)=-u_{2}\left(t_{n}\right), \quad w_{1}\left(t_{n+1}\right)=w_{2}\left(t_{n}\right), \quad \omega_{1}\left(t_{n+1}\right)=\omega_{2}\left(t_{n}\right),
$$

which with the help of (6-3) are used to determine the initial conditions $\left\{u_{2}\left(t_{n+1}\right), w_{2}\left(t_{n+1}\right), \omega_{2}\left(t_{n+1}\right)\right\}$ just after collision at $t=t_{n+1}$. Then, the process is repeated for additional collisions.

Before presenting the solution to bouncing, it is of interest to examine the dependence of the incident obliquity angle $\theta$ and the quantity $(1-\eta)$, which characterizes energy dissipation, on the parameter $z$ for different values of the model parameters. For the examples in this section $\mu_{s}$ is specified by the value in (5-2) and the solution is considered for different values of $e$ and $\alpha$. Specifically, use is made of (2-8), (3-10), (3-12), and (6-3) to determine values for $\theta$ and (1- 1 ). Figure 7 plots $\theta$ as a function of $z$ for different values of $e$. From this figure it can be seen that the obliquity angles $\theta$ for $e=1$ and 0.8 are greater than $60^{\circ}$ at the transition to full slip $(z=1)$ so the transition region with partial slip is significant. Figure 8 plots $(1-\eta)$ as a function of $z$ for different values of $e$ and $\alpha$. Recalling that $(1-\eta)$ must be positive for positive energy dissipation during the collision, it can be seen that for $e=1$, the value of $\alpha$ has to be less than or equal to unity for positive energy dissipation, but for lower values of $e$ the value of $\alpha$ can be greater than unity and still satisfy the restriction of positive energy dissipation. 
In order to interpret the results of specific examples, it is convenient to define the following normalized parameters

$$
\begin{array}{lll}
U_{n}=\frac{u_{1}\left(t_{n}\right)}{u_{1}\left(t_{1}\right)}, & W_{n}=\frac{w_{1}\left(t_{n}\right)}{u_{1}\left(t_{1}\right)}, & \Omega_{n}=\frac{R_{A} \omega_{1}\left(t_{n}\right)}{u_{1}\left(t_{1}\right)}, \\
S_{n}=\frac{s_{1}\left(t_{n}\right)}{\psi_{s} u_{1}\left(t_{1}\right)}=\frac{W_{n}+\Omega_{n}}{\psi_{s}}, & \bar{T}_{n}=\frac{T\left(t_{n}\right)}{\frac{1}{2} m_{A} u_{1}^{2}\left(t_{n}\right)}=U_{n}^{2}+W_{n}^{2}+\frac{2}{5} \Omega_{n}^{2}, & E_{n}=\frac{\bar{T}_{n}}{\bar{T}_{1}},
\end{array}
$$

where $\left\{U_{n}, W_{n}, \Omega_{n}, S_{n}, \bar{T}_{n}, E_{n}\right\}$ characterize, respectively, the vertical velocity, horizontal velocity, angular velocity, slip velocity, kinetic energy and the energy reduction just before the collision at $t=t_{n}$. Then, equations (6-3), (6-11) and (6-12) yield

$$
\begin{aligned}
& z_{n}=\frac{\left|S_{n}\right|}{U_{n}}, \quad g_{n}=1-\left\langle 1-z_{n}\right\rangle^{1+\alpha}, \quad \eta_{n}=-1+\left[\frac{1+e+\mu_{s} \psi_{s} g_{n}^{2}}{1+\mu_{s} \psi_{s} z_{n} g_{n}}\right], \\
& U_{n+1}=e U_{n}, \quad W_{n+1}=W_{n}-\frac{2}{7} \psi_{s}\left(\frac{S_{n}}{z_{n}}\right) g_{n}, \quad \Omega_{n+1}=\Omega_{n}-\frac{5}{7} \psi_{s}\left(\frac{S_{n}}{z_{n}}\right) g_{n}, \\
& S_{n+1}=S_{n} G_{n}, \quad G_{n}\left(z_{n}\right)=1-\frac{g_{n}}{z_{n}} .
\end{aligned}
$$

Next, consider special state defined by

$$
W_{n}=\frac{1}{7}(2+5 \gamma) \psi_{s} S_{n}, \quad \Omega_{n}=\frac{5}{7}(1-\gamma) \psi_{s} S_{n}, \quad \bar{T}_{n}=U_{n}^{2}+\frac{1}{7} \psi_{s}^{2} S_{n}^{2}\left(2+5 \gamma^{2}\right) .
$$

From these expressions it can be seen that the two states $\gamma= \pm|\gamma|$, with different values of the horizontal velocity $W_{n}$ and angular velocity $\Omega_{n}$, have the same slip velocity $S_{n}$ and the same kinetic energy $\bar{T}_{n}$. Moreover, it follows from (6-13) and (6-14) that

$$
\begin{aligned}
W_{n+1} & =\frac{1}{7}(2+5 \bar{\gamma}) \psi_{s} S_{n+1}, & \Omega_{n+1} & =\frac{5}{7}(1-\bar{\gamma}) \psi_{s} S_{n+1}, \\
\bar{T}_{n+1} & =U_{n+1}^{2}+\frac{1}{7} \psi_{s}^{2} S_{n+1}^{2}\left(2+5 \bar{\gamma}^{2}\right), & \bar{\gamma} & =\frac{\gamma}{1-\frac{g_{n}}{z_{n}}} .
\end{aligned}
$$

Comparison of (6-14) and (6-15) indicates that consecutive collisions maintain the same structure so the response of the slip velocity $S_{n}$ and the energy reduction $E_{n}$ for the two initial states specified by

$$
U_{1}=1, \quad W_{1}=\frac{1}{7}(2+5 \gamma) \psi_{s} S_{1}, \quad \Omega_{1}=\frac{5}{7}(1-\gamma) \psi_{s} S_{1}, \quad \bar{T}_{1}=1+\frac{1}{7} \psi_{s}^{2} S_{1}^{2}\left(2+5 \gamma^{2}\right),
$$

with $\gamma= \pm|\gamma|$ are the same. This means that the parameters in the model can be determined by experiments or simulations for which the sphere has no initial angular velocity $\{\gamma=1\}$ and the results can be used for general states. In the following examples the initial state is specified by (6-16) with

$$
S_{1}=z_{1}>0
$$

From (6-13) it follows that when $e$ is less than unity, the value of the vertical velocity $U_{n}$ decreases with each collision, eventually attaining the value of zero. The function $G_{n}\left(z_{n}\right)$ in (6-13) which characterizes the slip velocity satisfies the properties

$$
G_{n}(0)=-\alpha, \quad G_{n}(1)=0, \quad G_{n}(\infty)=1, \quad \frac{d G_{n}}{d z_{n}} \geq 0, \quad-\alpha \leq G\left(z_{n}\right) \leq 1 .
$$


Moreover, it can be shown that

$$
-1<G_{n}\left(z_{n}\right)<1 \text { for } 0<\alpha<1 \text { and } 0 \leq z_{n}<\infty
$$

which in view of (6-13) means that the slip velocity decreases during each collision as long as $z_{n}$ remains bounded. However, the rate of decrease in $U_{n}$ might be slower than that of $S_{n}$, causing $z_{n}$ to approach infinity and the value of $S_{n}$ to approach a non-zero constant.

6.1. Bouncing vertically with no slip. The simplest special solution is one where the sphere does not translate horizontally and does not rotate with

$$
z_{n}=0, \quad W_{n}=0, \quad \Omega_{n}=0, \quad S_{n}=0 \text { for } n=1,2, \ldots
$$

This solution is valid for any values of $\left\{e, \alpha, \mu_{s}\right\}$ and consists of the sphere merely bouncing vertically. If $e$ is less than unity, the sphere loses energy during each collision and eventually stops bouncing.

6.2. Bouncing vertically with initial slip. For this example the initial slip velocity has the special value

$$
S_{1}=z_{1}=1, \quad W_{1}+\Omega_{1}=\psi_{s},
$$

which allows the sphere to initially have nonzero horizontal velocity $W_{1}$ and nonzero angular velocity $\Omega_{1}$. Since $G_{n}(1)=0$ in (6-13) it follows that slip velocity $S_{n}$ reduces to zero during the first collision and remains zero

$$
S_{n}=0 \text { for } z_{1}=1 \text { and } n=2,3, \ldots
$$

This result is also independent of the values of $\{e, \alpha\}$. Although the slip velocity might be zero this does not mean that the sphere has stopped rotating or translating. In particular, it can be seen from (6-14)-(6-15) that $\left\{W_{n}, \Omega_{n}\right\}$ will both vanish when $S_{n}$ vanishes if

$$
\gamma=0, \quad \frac{W_{n}}{\Omega_{n}}=\frac{2}{5} \text { for } n=1,2, \ldots
$$

6.3. Bouncing with partial energy dissipation. For an example of bouncing with partial energy dissipation, the parameters are specified by (6-16) and (6-17) with

$$
e=1, \quad \alpha=0.35, \quad \mu_{s}=0.3, \quad \gamma=1, \quad z_{1}=0.5
$$

Figure 9 shows the response of this sphere which is initially not rotating. Since $e=1$, the vertical velocity $U_{n}$ before each collision shown in part (a) of the figure remains constant so there is only partial energy dissipation. The slip velocity $S_{n}$ in part (b) reduces to zero, the horizontal velocity $W_{n}$ in part (c) attains a constant positive value and the energy reduction $E_{n}$ in part (d) decreases to a constant positive value. The sphere continues to bounce, translating and rotating without slip at the contact points of the collisions.

6.4. Bouncing with dissipation of the vertical kinetic energy. For an example of bouncing with dissipation of the vertical kinetic energy, the parameters are specified by

$$
e=0.8, \quad \alpha=0.35, \quad \mu_{s}=0.3, \quad \gamma=0, \quad z_{1}=1
$$



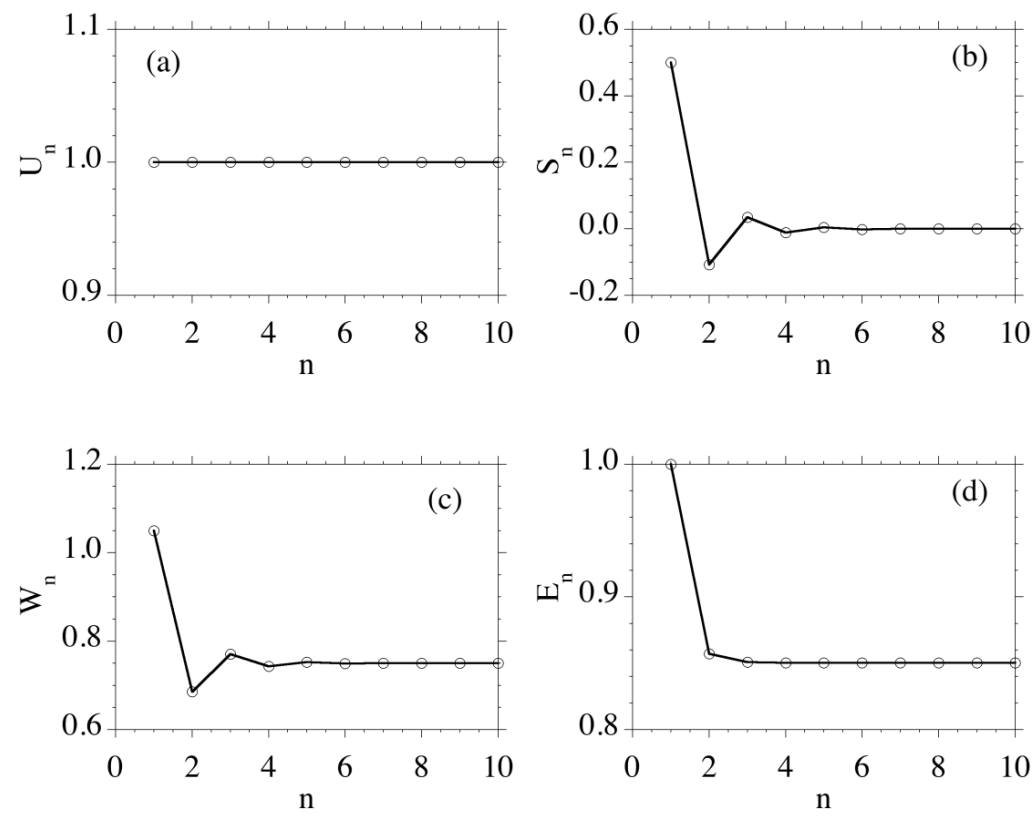

Figure 9. Bouncing with partial energy dissipation: The solution shows initial slip with $\left\{S_{n}, W_{n}\right\}$. The parameters are specified by $e=1, \alpha=0.35, \mu_{s}=0.3, \gamma=1, z_{1}=0.5$.

Figure 10 shows that for these special values $\left\{\gamma=0, z_{1}=1\right\}$ the nonzero initial values of the slip velocity $S_{n}$, the horizontal velocity $W_{n}$ and the angular velocity $\Omega_{n}$ all drop to zero during the first collision and remain zero. This means that after the first collision the sphere bounces vertically until all its kinetic energy is dissipated.

6.5. Bouncing with dissipation of the vertical kinetic energy and continued slip. For an example of bouncing with dissipation of the vertical kinetic energy and continued slip the parameters are specified by

$$
e=0.8, \quad \alpha=0.35, \quad \mu_{s}=0.3, \quad \gamma=1, \quad z_{1}=6
$$

which has a much larger value of the slip velocity and obliquity angle than that in (6-25). Figure 11(a) shows that the normal velocity $U_{n}$ decays to zero; in the rest of the figure it can be seen that the slip velocity $S_{n}$ (b), the horizontal velocity $W_{n}$ (c) and the energy reduction $E_{n}(\mathrm{~d})$ all reduce to positive values. In particular, the normal velocity $U_{n}$ decays faster than the slip velocity $S_{n}$, causing $z_{n}$ to approach infinity with $G_{n}$ in (6-13) approaching zero. Also, it is interesting to note that when $z_{n}$ approaches infinity the parameter $\eta$ approaches $(-1)$.

As $U_{n}$ approaches zero the sphere chatters (bouncing with imperceptible amplitude). The kinetic energy remains constant even though the sphere continues to slip because the force of gravity is neglected during these small collision events. This is an unphysical result of the approximation that the impulsive force dominates the bounded force of gravity during the collision and it identifies a limitation of the model for certain situations with multiple collision events. 

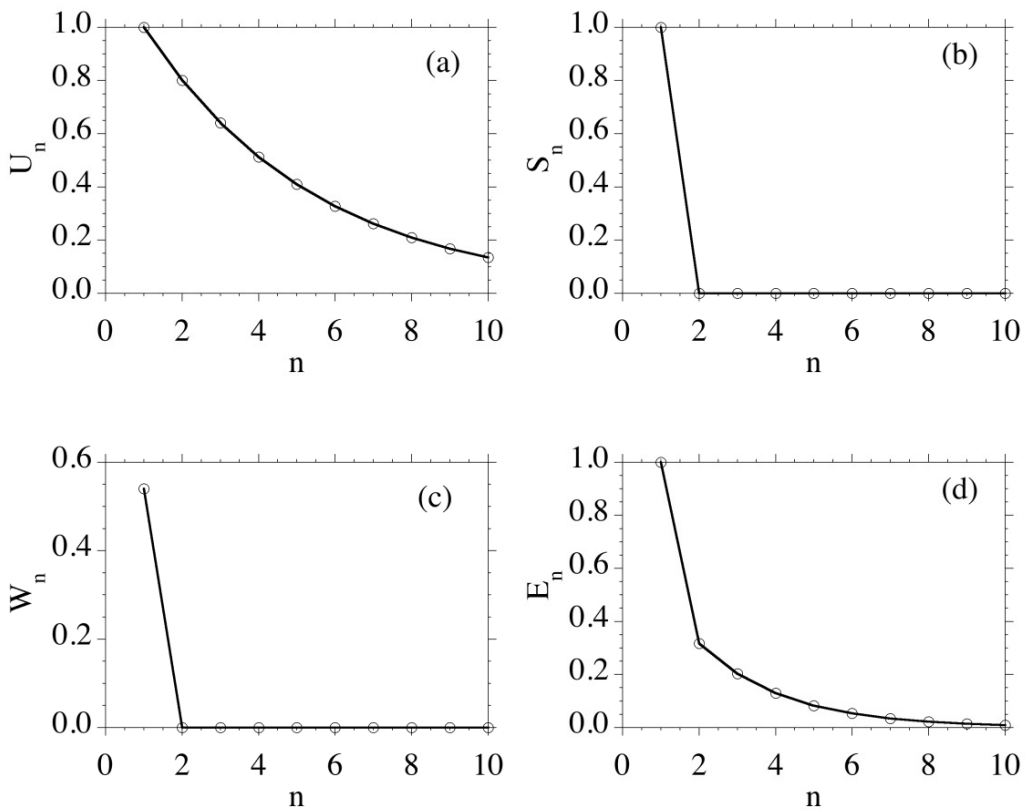

Figure 10. Bouncing with dissipation of the vertical kinetic energy: The solution shows initial slip with $\left\{S_{n}, W_{n}, \Omega_{n}\right\}$ dropping to zero after the first collision. The parameters are specified by $e=0.8, \alpha=0.35, \mu_{s}=0.3, \gamma=0, z_{1}=1$.
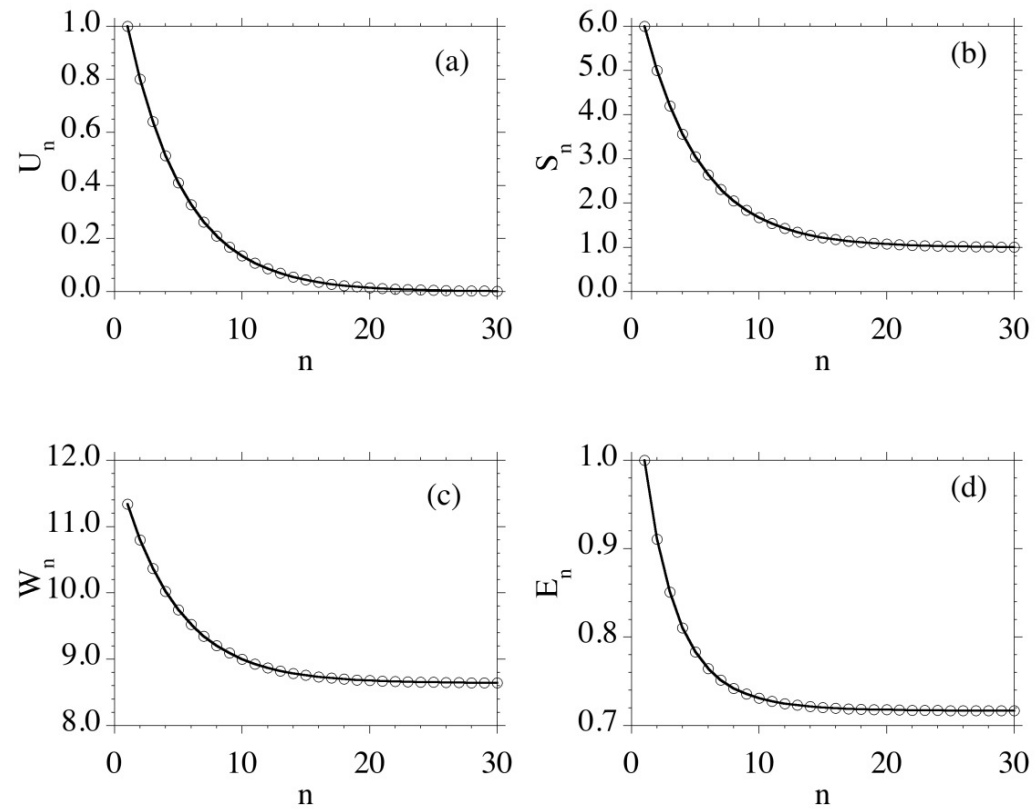

Figure 11. Bouncing with dissipation of the vertical kinetic energy: The solution shows continued slipping $\left(S_{n}>0\right)$ with the parameters specified by $e=0.8, \alpha=0.35, \mu_{s}=0.3$, $\gamma=1, z_{1}=6$. 


\section{Conclusions}

The smooth transition model for hard-particle collision of spheres presented here has been motivated by the model of Walton [1993], which has a sharp transition between a region of partial slip for low obliquity angles and a region of full slip for higher obliquity angles. Specifically, the notion of a coefficient of rotational restitution in the Walton model is replaced by a constitutive equation for the shear coefficient $\mu$, which controls the ratio of the shear component of the impulsive force to its normal component. The model depends on three parameters: the standard coefficient of restitution $e$ for relative normal velocities; a constant value $\mu_{s}$ of friction for sliding in the full slip region; and a parameter $\alpha$ in the function $g(z)$ in (3-12) that controls the shape of the transition region from partial to full slip.

The resulting smooth transition model is relatively simple and the three model parameters $\left\{e, \mu_{s}, \alpha\right\}$ can be evaluated from experimental data or from simulations using soft-particle contact models. Examples show that the smooth transition region is large with the obliquity angle $\theta$ being between 0 and nearly $60^{\circ}$. Moreover, it is shown that the physical condition of non-negative energy dissipation imposes nontrivial restrictions on the model parameters.

\section{Acknowledgements}

The author would like to acknowledge helpful discussions with $\mathrm{O}$ Walton. Also, this research was partially supported by MB Rubin's Gerard Swope Chair in Mechanics.

\section{References}

[Chatterjee and Ruina 1998] A. Chatterjee and A. Ruina, "A new algebraic rigid-body collision law based on impulse space considerations”, J. Appl. Mech. (ASME) 65:4 (1998), 939-951.

[Deen et al. 2007] N. G. Deen, M. Van Sint Annaland, M. A. Van der Hoef, and J. A. M. Kuipers, "Review of discrete particle modeling of fluidized beds", Chem. Eng. Sci. 62:1-2 (2007), 28-44.

[Di Renzo and Di Maio 2004] A. Di Renzo and F. P. Di Maio, "Comparison of contact-force models for the simulation of collisions in DEM-based granular flow codes”, Chem. Eng. Sci. 59:3 (2004), 525-541.

[Goldsmith 1960] W. Goldsmith, Impact: the theory and physical behaviour of colliding solids, Edward Arnold, London, 1960.

[Ivanov 1992] A. P. Ivanov, “Энергетика удара с трением”, Prikl. Mat. Mekh. 56:4 (1992), 624-631. Translated as "Energetics of a collision with friction" in J. Appl. Math. Mech. 56:4 (1992), 527-534.

[Ketterhagen et al. 2005] W. R. Ketterhagen, J. S. Curtis, and C. R. Wassgren, "Stress results from two-dimensional granular shear flow simulations using various collision models", Phys. Rev. E 71:6 (2005), Article ID \#061307.

[Kosinski and Hoffmann 2010] P. Kosinski and A. C. Hoffmann, "An extension of the hard-sphere particle-particle collision model to study agglomeration”, Chem. Eng. Sci. 65:10 (2010), 3231-3239.

[Kosinski and Hoffmann 2011] P. Kosinski and A. C. Hoffmann, "Extended hard-sphere model and collisions of cohesive particles", Phys. Rev. E 84:3 (2011), Article ID \#031303. Erratum in 89:4 (2014), Article ID \#049901.

[Kruggel-Emden et al. 2007] H. Kruggel-Emden, E. Simsek, S. Rickelt, S. Wirtz, and V. Scherer, "Review and extension of normal force models for the discrete element method", Powder Technol. 171:3 (2007), 157-173.

[Liu et al. 2011] G. Liu, S. Li, and Q. Yao, "A JKR-based dynamic model for the impact of micro-particle with a flat surface", Powder Technol. 207:1-3 (2011), 215-223.

[Lu et al. 2005] H. Lu, S. Wang, Y. Zhao, L. Yang, D. Gidaspow, and J. Ding, "Prediction of particle motion in a twodimensional bubbling fluidized bed using discrete hard-sphere model”, Chem. Eng. Sci. 60:12 (2005), 3217-3231.

[Maw et al. 1976] N. Maw, J. R. Barber, and J. N. Fawcett, “The oblique impact of elastic spheres", Wear 38:1 (1976), $101-114$. 
[Maw et al. 1981] N. Maw, J. R. Barber, and J. N. Fawcett, "The role of elastic tangential compliance in oblique impact", J. Lubr. Technol. (ASME) 103:1 (1981), 74-80.

[Meyer and Deglon 2011] C. J. Meyer and D. A. Deglon, "Particle collision modeling: a review", Miner. Eng. 24:8 (2011), 719-730.

[Morris et al. 2004] J. P. Morris, M. B. Rubin, S. C. Blair, L. A. Glenn, and F. E. Heuze, "Simulations of underground structures subjected to dynamic loading using the distinct element method", Eng. Comput. (Swansea Wales) 21:2-4 (2004), 384-408.

[Prasanth et al. 2012] P. S. Prasanth, J. K. Kakkassery, and R. Vijayakumar, "A variable hard sphere-based phenomenological inelastic collision model for rarefied gas flow simulations by the direct simulation Monte Carlo method", Fluid Dyn. Res. 44:2 (2012), Article ID \#025503.

[Rubin 1998] M. B. Rubin, "Physical restrictions on the impulse acting during three-dimensional impact of two 'rigid' bodies", J. Appl. Mech. (ASME) 65:2 (1998), 464-469.

[Stevens and Hrenya 2005] A. B. Stevens and C. M. Hrenya, "Comparison of soft-sphere models to measurements of collision properties during normal impacts", Powder Technol. 154:2-3 (2005), 99-109.

[Walton 1993] O. R. Walton, "Numerical simulation of inelastic frictional particle-particle interactions", Chapter 25, pp. 884911 in Particulate two-phase flow, edited by M. C. Roco, Butterworth-Heinemann, Boston, 1993.

[Walton 2004] O. R. Walton, "Potential discrete element simulation applications ranging from airborne fines to pellet beds", SAE Technical Paper 2004-01-2329, 2004.

[Zhu et al. 2007] H. P. Zhu, Z. Y. Zhou, R. Y. Yang, and A. B. Yu, "Discrete particle simulation of particulate systems: theoretical developments", Chem. Eng. Sci. 62:13 (2007), 3378-3396.

Received 23 May 2015. Accepted 20 Sep 2015.

M. B. RUBIN: mbrubin@tx.technion.ac.il

Faculty of Mechanical Engineering, Technion - Israel Institute of Technology, 32000 Haifa, Israel 



\title{
MULTIOBJECTIVE OPTIMIZATION OF LAMINATED COMPOSITE PLATE WITH ELLIPTICAL CUT-OUT USING ANN BASED NSGA-II
}

\author{
P. Emmanuel Nicholas, M. C. Lenin Babu And A. Sathya Sofia
}

\begin{abstract}
Laminated composites are highly in demand for the applications where high strength and stiffness are required at less weight. They generally fail due to buckling, as they are modeled as thin plates and are loaded compressively. Therefore, the design parameters of the laminated composite plates are to be optimized for the multiple-conflicting objectives buckling strength and weight. However, the composite plates, which are used in real world applications, are to be made with cut-outs and finite element analysis is required to analyze them. As it makes the optimization process more complex, a methodology is proposed in this paper to carry out a multiobjective optimization for the rectangular composite plate made with a central elliptical cut-out. The nondominated solutions are obtained using nondominated sorting genetic algorithm (NSGA-II) in which the multilayer feed-forward neural network is used to replace the time consuming finite element analysis. The numerical results show that the proposed method finds the nondominated solutions efficiently and reduces the computational cost prominently.
\end{abstract}

\section{Introduction}

Fiber reinforced laminated composite structures have high specific strength and stiffness and so, they are widely used in aerospace industries, automobile industries and wind turbine blades. In those applications, they are generally modeled as thin plates and shells and are compressively loaded. Hence, they generally fail due to buckling and it is mandatory to optimize the design variables of the composite plates for the multiple objectives such as maximum buckling strength and minimum weight. Aymerich and Serra [2008] optimized conventional ply angles $\left(0^{\circ} / \pm 45^{\circ} / 90^{\circ}\right)$ to increase the buckling strength of the composite plate. Almeida and Awruch [2009] carried out a multiobjective optimization to minimize the weight and deflection of the composite plate. The conventional ply angles were used as design variables and finite element method was used to evaluate the buckling strength of the structure. Omkar et al. [2009; 2011] have optimized the composite plate for the multiple objectives weight and the total cost. These multiobjective optimization problems were solved in the similar fashion of the single objective optimization problem by assigning the suitable weights to the objective functions. However, the exact values of the weights cannot be obtained, as the importance of each objective function cannot be clearly quantified in the multiobjective optimization problems. Further, the ply angles having the same orientation are grouped in the optimum stacking sequences, as conventional ply angles are generally optimized to improve the structural behavior of the composites. Kim et al. [2005] and Kim [2007] stated that the thickness of the ply having a particular orientation is increased when the plies having the same orientation are stacked together. They also mentioned that the interlaminar normal and shear stresses are developed due to the grouping of plies having the same orientation. Campbell [2010] and Emmanuel Nicholas et

Keywords: stacking sequence optimization, artificial neural network, NSGA-II, finite element analysis. 
al. [2014] recommend for maintaining the dispersed stacking sequences so as to avoid grouping of plies having the same orientation.

In real world applications, it is indispensable to design the composite plates with cut-outs (holes) in order to provide fasteners to assemble them as a complex structure. In aircraft industries, the cut-outs are necessary to function as doors, windows and access ports. Al Qablan et al. [2009] stated that the cut-outs are necessary for accessibility reasons and to reduce the weight of the composite structure. The finite element analysis was used to find the buckling strength of the composite plate with cut-outs. Liu et al. [2006] indicated that the design of interior cut-outs in laminated composite structures is of great importance in the aerospace, automobile and structural engineering. Lopes et al. [2010] mentioned that the cut-outs on composite structures are required to accommodate windows, doors, and bolted joints. Aydin Komur et al. [2010] referred that the cut-outs are generally used in composites as design necessities. They analyzed the buckling strength of a woven-glass-polyester laminated composite plate with circular/elliptical cutout. The finite element method was used to study the effect of the cutout shape on the buckling strength. Erklig and Yeter [2012] used finite element analysis to study the effects of various cut-out shapes on the lateral buckling behavior of composite beams. Iyengar and Vyas [2011] carried out an optimization process to maximize the buckling load of the laminated composite plate with and without cut-out. However, the design space was restricted by using very few ply angles $\left( \pm 60^{\circ}, \pm 45^{\circ}, \pm 30^{\circ}, 0^{\circ} / 90^{\circ}\right)$ as the design variables. Liu et al. [2006] and Lopes et al. [2010] performed the shape optimization of multiple interior cutouts by varying $[\theta /-\theta]_{2 s}$ stacking sequences. Rocha et al. [2014] considered the laminated composite curved panel with a central circular cutout that is generally used in aerospace applications. He optimized the structure consists of 8 plies by varying the conventional ply angles and ply thickness.

The artificial neural network is generally used as a prediction tool in the applications, where the opportunities are not available or not possible to find the actual targets. Recently, ANN has been successfully used to approximate the mechanical behaviors of the laminated composites. Zheng et al. [2009] successfully used the wavelet neural network to estimate the delamination locality. Bilgehan [2011] used artificial neural network to analyze the buckling strength of slender prismatic columns. Chakraborty [2005] used natural frequencies as inputs to the artificial neural network and predicted the existence of embedded delaminations based on their size, shape and location in FRP composites. The natural frequencies of laminated composite for different blends of size, shape and location of an embedded delamination were obtained using finite element method and these samples have been used to train a back propagation neural network (BPNN) to predict the delaminations in the composite structure. Karnik et al. [2008] carried out delamination analysis in high speed drilling with the application of artificial neural network model. The spindle speed, feed rate and point angle were used as the input parameters to the network structure.

Tsao and Hocheng [2008] used radial basis function network (RBFN) and predicted the thrust force and surface roughness in composite materials during the drilling process. Al-Assaf and El Kadi [2001] applied ANN to predict the mechanical properties of fiber reinforced polymeric composites. They examined the possibility of using neural network to predict the failure due to fatigue. The ply angle, stress ratio and maximum stress were given as the inputs to the network and the number of cycles to failure has been found as the output. Al-Haik et al. [2006] studied the visco-plastic behavior of composites using a neural networks formulation. Mishra et al. [2010] used artificial neural network to predict the residual tensile strength of unidirectional glass fiber reinforced plastic laminates with drilled hole. The drill point geometry, feed rate and the spindle speed have been used as the input variables to the network. 
The number of neurons in the input and output layers were equal to the number of input and output parameters. The number of neurons in the hidden layer was optimally found based on trial and error method. Reddy et al. [2013] used artificial neural networks to predict the deflection and stresses of carbon fiber reinforced plastic (CFRP) laminated composite square plate subjected to uniformly distributed load. Cardozo et al. [2011] used ANN based optimization method to optimize the stacking sequence of laminated composite. However, the work is limited by using only the conventional ply angles.

It is observed from the literature review that many research works have been done on stacking sequence optimization of rectangular laminated composite plate to increase the buckling strength and reduce the weight of the structure. The in-plane and buckling strengths of the rectangular composite plate were computed analytically during the optimization process. However, the literature review also reveals that the composite plate, which is to be used in real world applications, has to be made with cut-out. Further, it is exposed that the plate made with cut-out has to be analyzed using FEM and this increases the computational cost. Though many researchers used FEM and ANN and studied the influences of cutout size, shape and its location on buckling strength and other mechanical behaviors of the composite plate, the design factors of the composite plate made with cut-out have not been optimized adequately. In addition, the conventional ply angles have been generally used as the design variables and also the complex multiobjective optimization problems have been solved like the single objective optimization problems. Hence, the ANN based methodology is proposed in this paper to obtain the Pareto-optimal designs for the laminated composite plate made with cut-out, whereas the design space is critically increased with the choice of reduced ply angle intervals.

\section{Problem definition and solution methodology}

2.1. Problem definition. The rectangular composite plate with central elliptical cut-out shown in Figure 1 is considered in this research work whereas the cut-out is oriented at an angle $60^{\circ}$ from the major coordinate axis. The simply supported boundary conditions and biaxial compressive loads are applied on all the four sides of the plate. The ply angle $(\theta)$, number of plies (n) and stacking sequence are chosen as design variables and the in-plane strengths obtained using Tsai-Wu criterion is set as design constraint. The design space is critically increased using the concept of ply angles having the reduced intervals so as to improve the degree of the objective functions and to reduce the number of grouping of plies having the same orientation. The objective functions, design variables and constraints are defined in Equation (1).

Maximize the buckling strength $\lambda_{b}=f\left(\left[\theta_{1} / \theta_{2} / \theta_{3} \ldots / \theta_{n}\right]\right)$

and minimize the weight (total thickness) $T=\sum_{i=1}^{k} t_{i}$

subject to $\theta_{i}=-90^{\circ}$ to $90^{\circ}$ with an increment " $\Delta$ "

$t_{i}=0$ or $0.125 \mathrm{~mm}$

$f_{1} \sigma_{11} i+f_{2} \sigma_{22}+f_{6} \sigma_{12}+f_{11} \sigma_{11}^{2}+f_{22} \sigma_{22}^{2}++f_{66} \sigma_{12}^{2}+2 f_{12} \sigma_{11} \sigma_{22} \leq 1$

$G \leq 4$

where $n=$ number of plies in the plate

$t_{i}=$ thickness of each ply " $i$ "

$\Delta=$ any value of $45^{\circ}, 15^{\circ}, 5^{\circ}$ and $1^{\circ}$

$i=1,2,3, \ldots, n / 2$ (i.e., stacking sequence is symmetric)

and $G=$ number of continuous plies having the same orientation. 
2.2. Solution methodology. The artificial neural network is considered in the optimization procedure to predict the buckling strength of the laminated composite plate made with cut-out. The proposed optimization method comprises of three modules. In the first module, the stacking sequences of the laminated plate made with cut-out are randomly generated using MATLAB code and buckling strength

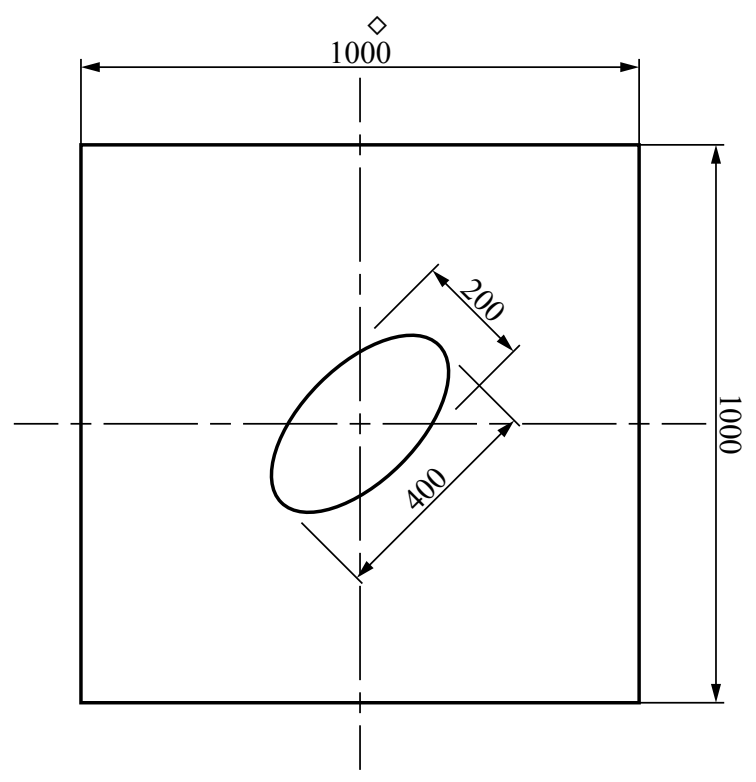

Figure 1. Geometry of the structure.

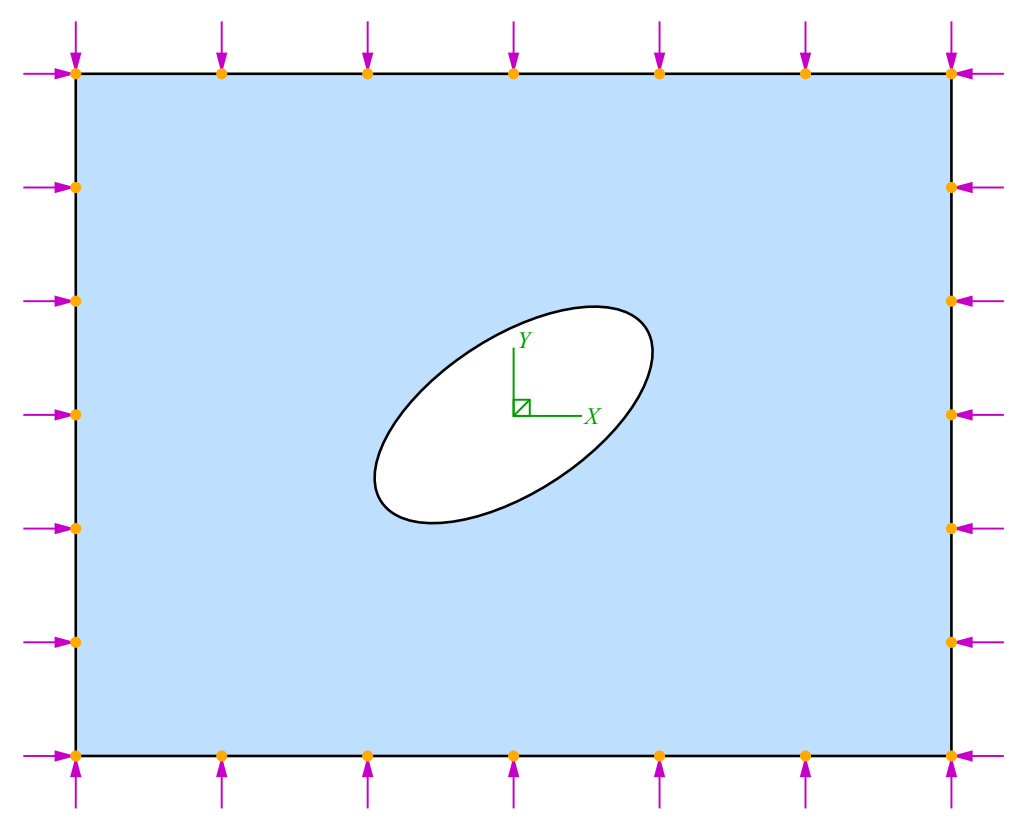

Figure 2. Loading and boundary conditions. 


\begin{tabular}{|c|c|}
\hline Property & Values \\
\hline$E_{1}$ & $133.86 \mathrm{GPa}$ \\
$E_{2}=E_{3}$ & $7.706 \mathrm{GPa}$ \\
$G_{12}=G_{13}$ & $4.306 \mathrm{GPa}$ \\
$G_{23}$ & $2.76 \mathrm{GPa}$ \\
$v_{12}=v_{13}$ & 0.301 \\
$v_{23}$ & 0.396 \\
$V_{\mathrm{f}}$ & 0.55 \\
$F_{1 \mathrm{~T}}$ & $1500 \mathrm{MPa}$ \\
$F_{1 \mathrm{C}}$ & $1200 \mathrm{MPa}$ \\
$F_{2 \mathrm{~T}}$ & $40 \mathrm{MPa}$ \\
$F_{2 \mathrm{C}}$ & $246 \mathrm{MPa}$ \\
$F_{6}$ & $68 \mathrm{MPa}$ \\
\hline
\end{tabular}

Table 1. Material properties of the (AS4D/9310) carbon/epoxy composite.

of each configuration is evaluated using the commercial finite element analysis software ABAQUS. In the second module, the neural network (ANN) structure is constructed using MATLAB code and it is trained using the sample data generated in the first module. The genetic algorithm, in which the trained ANN is used to predict the fitness values, is used as the optimization tool in the third module.

2.3. Finite element analysis and data generation. The finite element method is used to analyze the structure when the geometry, material property or loading condition is irregular. The commercial finite element analysis software ABAQUS is used in this paper to equip the sample data so as to train and test the ANN. The stacking sequences of the rectangular composite plate made with the elliptical cut-out are randomly generated from the specified design space and the buckling strength and the ply stresses (through which the safety factor is found using Tsai-Wu criterion) of each configuration are calculated using ABAQUS. The MATLAB code is used to generate the stacking sequences randomly and python script is used to interface ABAQUS with MATLAB. The material properties listed in Table 1 are applied to the plate. The maximum number of plies is set as 24 , whereas each ply has a uniform thickness of 0 or $0.125 \mathrm{~mm}$. The value of ply thickness is set as 0 so as to vary the number of plies.

The simply supported boundary conditions (as given in Equation (2)) and biaxial compressive load are applied along the edges of the plate as shown in Figure 2. The finite element model of the plate is generated using four node shell element (S4R) where each node has six degrees of freedom. The sample finite element model of the plate with cut-out is shown in Figure 3. In this paper, the number of elements is optimally found as 5300 by using the convergence graph shown in Figure 4.

$$
\begin{aligned}
& w_{0}(x, 0)=0, \quad w_{0}(x, b)=0, \quad w_{0}(0, y)=0, \quad w_{0}(a, y)=0, \\
& M_{x x}(0, y)=0, \quad M_{x x}(a, y)=0, \quad M_{y y}(x, o)=0, \quad M_{y y}(x, b)=0 .
\end{aligned}
$$

2.4. Artificial neural network. As the design space of this work is critically increased and FEA is required to analyze the plate, ANN is used in this paper to predict the buckling strength and the safety 


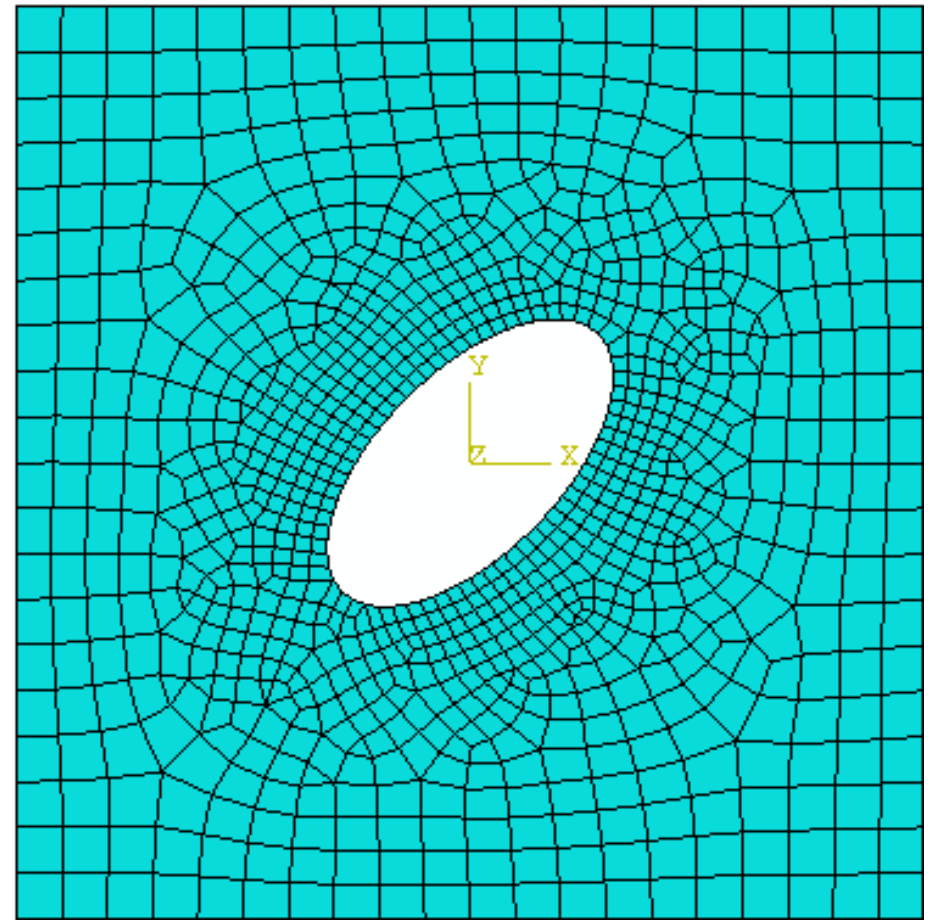

Figure 3. Finite element model of the composite plate with cut-out.

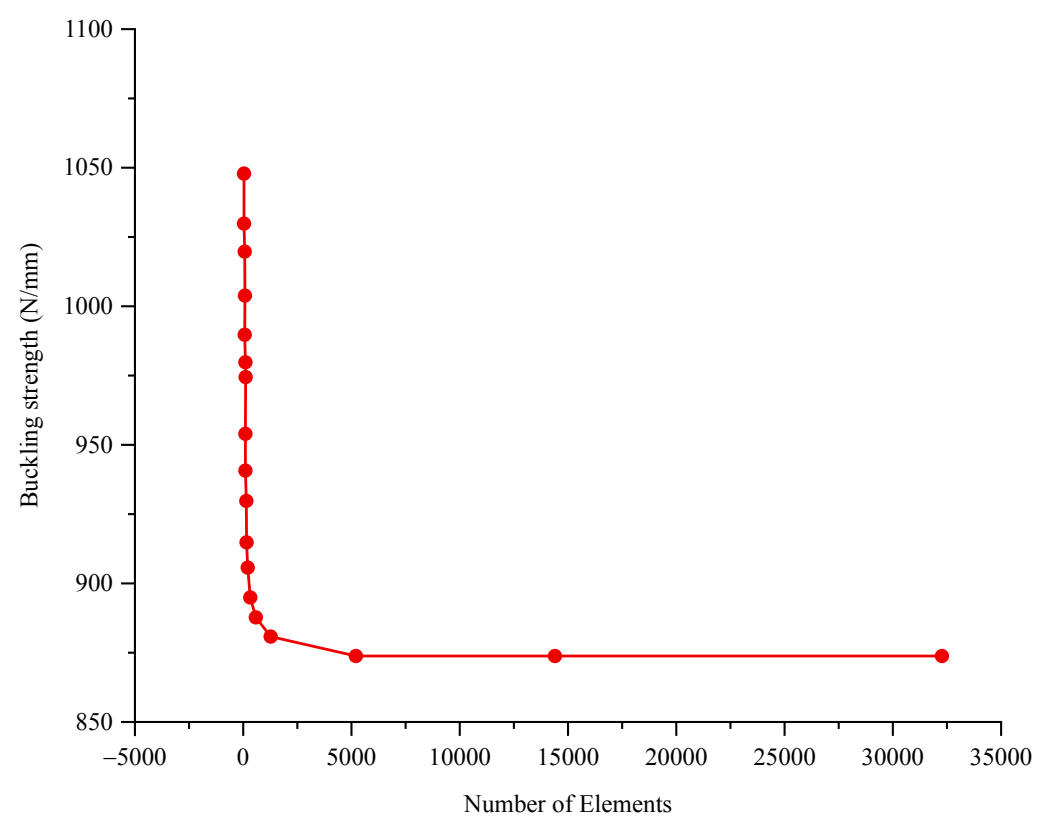

Figure 4. Convergence of number of elements. 


\begin{tabular}{|c|l|c|}
\hline Sl.No & Training Algorithm & Acronym \\
\hline 1 & resilient backpropagation & RP \\
2 & scaled conjugate gradient & SCG \\
3 & conjugate gradient with Powell/Beale restarts & CGB \\
4 & Fletcher-Powell conjugate gradient & CGF \\
5 & Polak-Ribière conjugate gradient & CGP \\
6 & Levenberg-Marquardt & LM \\
7 & BFGS quasi-Newton & BFG \\
\hline
\end{tabular}

Table 2. Various training algorithms.

factor during the optimization process. A multilayer feed-forward back- propagation neural network is used. The neural network is built in four stages. In the first stage, the sample data with their targets are collected. The network object with suitable neuron in each layer, number of layers, transfer function for each layer and the training algorithm of the network are chosen in the second stage. The created network is trained in stage three with the samples generated in stage one. The network is tested in stage four with the remaining samples and after the satisfaction of the performance of the network with these test samples, the network is simulated for the new inputs.

The performance of the neural network during the training and after the training periods highly depends on the choice of the network objects such as the numbers of hidden layers, number of neurons, transfer functions and the training algorithm. Yuen and Lam [2006] mentioned that these parameters are to be decided based on the experience or rule of thumb only. Bolanča et al. [2014] obtained the network structure by optimally varying these parameters. Kermanshahi and Iwamiya [2002] and Chakraborty [2005] also recommended for finding the network objects using the trial and error method. Four layers network is chosen for this work with the neurons 24, 30, 15 and 2, respectively. The input and output layers consist of 24 and 2 neurons, respectively, as the ANN has 24 inputs (layer angles) and 2 outputs (buckling strength and safety factor). The number of neurons in the hidden layers, transfer functions and the training algorithm are chosen based on the trial and error method [Mishra et al. 2010]. Since the design space is increased here with the choice of dispersed layer angles, 1000 samples of stacking sequences with their targets are generated for each ply angle interval. The inputs and the targets are normalized between -1 to 1 . Tan-sigmoid transfer function is used for the first three layers and linear transfer function is used in the last layer. Seven networks having same number of layers, neurons and same type of transfer functions have been constructed and they have been trained separately using the various training algorithms given in the Table 2 .

After the successful training, the networks were validated using the new data. Further, the average and the maximum error have also been found for each training algorithm and they are compared in Figure 5. It shows that the error level is high for the network trained by BFG type training algorithm and less for the network trained by CGF type training algorithm. Similarly, the average error rate is found to be maximum for the network trained by BFG type training algorithm and minimum for the network trained by CGF type training algorithm. Hence, the CGF type training algorithm is selected to train the network. The architecture of ANN is shown in Table 3. 

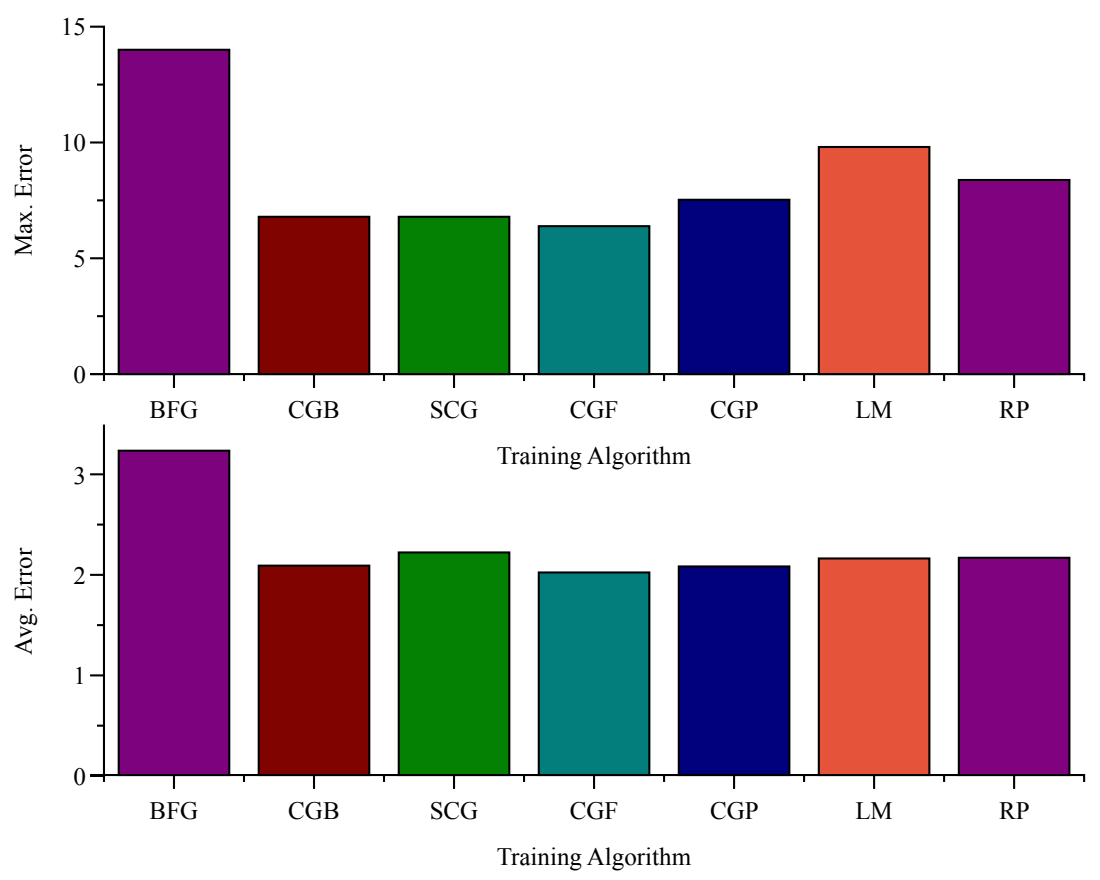

Figure 5. Networks error comparisons.

\begin{tabular}{|c|c|c|c|}
\hline Ply angle interval & Neurons in each layer & Transfer functions & Training algorithm \\
\hline $45^{\circ}$ & $24-30-15-2$ & Tansig-Tansig-Tansig-Linear & FRCGA $^{\dagger}$ \\
$30^{\circ}$ & $24-30-15-2$ & Tansig-Tansig-Tansig-Linear & FRCGA $^{\dagger}$ \\
$15^{\circ}$ & $24-30-15-2$ & Tansig-Tansig-Tansig-Linear & FRCGA $^{\dagger}$ \\
$5^{\circ}$ & $24-30-15-2$ & Tansig-Tansig-Tansig-Linear & FRCGA $^{\dagger}$ \\
\hline
\end{tabular}

Table 3. Architecture of ANN. ${ }^{\dagger}$ Fletcher-Reeves conjugate gradient algorithm.

2.5. Nondominated sorting genetic algorithm. The nondominated sorting genetic algorithm (NSGAII), proposed by Deb [2001], is used in this research work to obtain the Pareto optimal designs of the multiobjective optimization problem. It comes under the category of elitist multiobjective evolutionary algorithm. As the name suggests, an elite-preserving operator favors the elites of a population by giving them an opportunity to be directly carried over to the next generation. In this way, a good solution found at the beginning of the run will never be removed unless a better solution is found out.

Initially, the population is randomly generated and the solutions are sorted into each front based on nondomination. The dual objectives in the multiobjective optimization problem are maintained by using a fitness assignment scheme that prefers nondominated solutions. In the current population, the very first front is absolutely a nondominant set and the second front is dominated only by the solutions available in the first front and the sorting of fronts happen so on. A solution "S1" is said to dominate another solution "S2", if the following conditions are completely satisfied: 
- The solution $\mathrm{S} 1$ is not inferior to $\mathrm{S} 2$ in all the objectives.

- The solution S1 is strictly better than S2 in at least one objective.

A rank (fitness) value is assigned to the individuals of each front based on the front number in which they exist. Solutions available in the first front are assigned with a fitness value of 1 and solutions of the second front are assigned with a fitness value of 2 and so on. In addition to the fitness value, a factor called crowding distance is also calculated for each solution. The main objective of finding the crowding distance is to show the closeness of a solution to the ones of its neighbours. The systematic procedure of finding the crowding distance is given below.

- Let "P" be the number of solutions existing in a front and " $\mathrm{M}$ " the number of design objectives.

- For each objective function, the solutions in the front are sorted in the worst order of its objective values (fm) and the sorted indices vector ( Im) is found (usually the size of "I" is equal to the size of "P").

- A large distance values are assigned to the boundary solutions as given in (3).

$$
d_{1}^{m}=d_{p}^{m}=\infty
$$

- For the remaining solutions $(j=2$ to $P-1)$, the crowding distance is calculated using (4).

$$
d_{j}^{m}=I_{j}^{m}+\frac{I_{j+1}^{m}-I_{j-1}^{m}}{f_{\max }^{m}-f_{\min }^{m}} .
$$

The parents are selected from the current population based on their rank and crowding distance by using the tournament selection. The solution having a lower rank than another solution is selected. If both solutions are having the same rank, then the crowding distance is used to select the best solution. The solution with higher crowding distance is selected to the mating pool to reproduce the offspring. The uniform crossover and mutation operators are applied to generate the next populations. As the number of plies is also included as one of the design variables, the size of the chromosome in the multiobjective optimization is increased twice than that of the size used in the single objective optimization. The processes of uniform crossover and mutation applied in the multiobjective optimization problem are given in Tables 4 and 5 .

The symmetric laminated composite plate having maximum of eight plies above or below to the mid-plane is considered in this example. As number of plies, angle of each ply and stacking sequence are optimized in the multiobjective optimization problem, each chromosome has sixteen genes in this example. The genes in first half of the chromosome represent the ply angle, whereas the genes in the next half of the chromosome represent the ply thickness. The genes in the second half of the chromosome remain unchanged during the crossover and mutation processes, as each ply has a constant thickness of $0.1 \mathrm{~mm}$.

A distinct operator, named as ply deletion operator, is additionally used in the multiobjective optimization to vary the number of plies of the laminated composite plate. The ply deletion operator is used to take out a gene from the second half of the chromosome and to replace it with an empty stack code. This operator is applied based on the probability given by the user. If the randomly generated number is greater than the user given probability, the thickness of any ply is randomly chosen and set as zero. An example for the ply deletion operator is given in Table 5. 


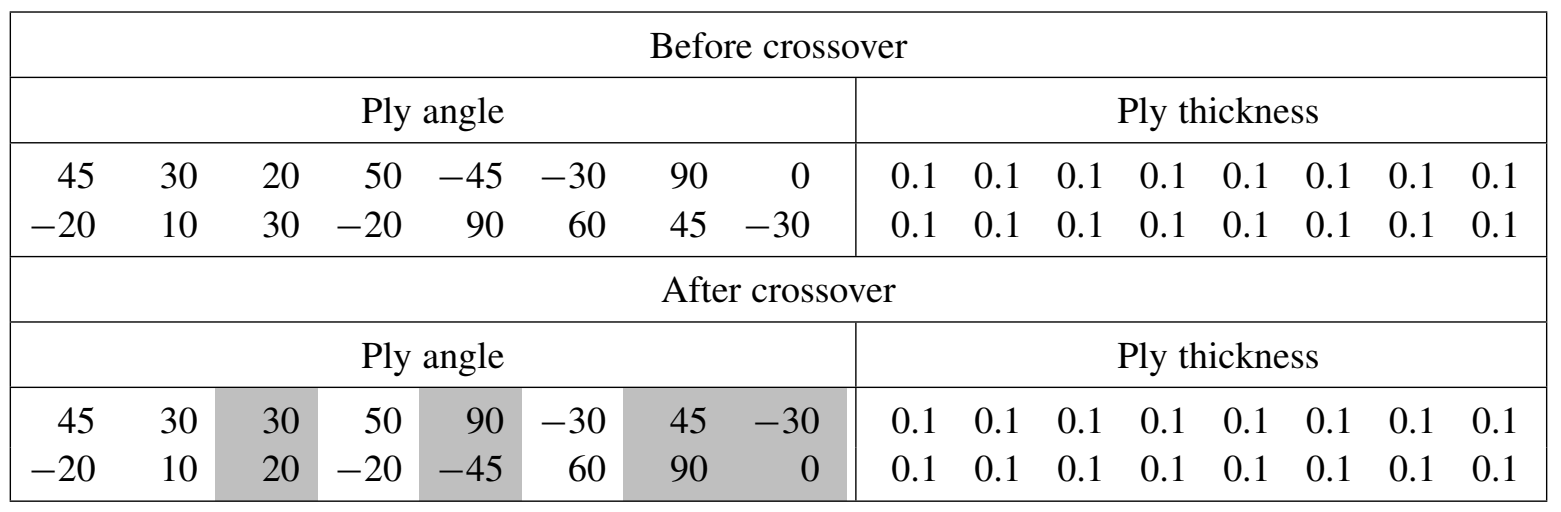

Table 4. An example of uniform crossover for NSGA-II.

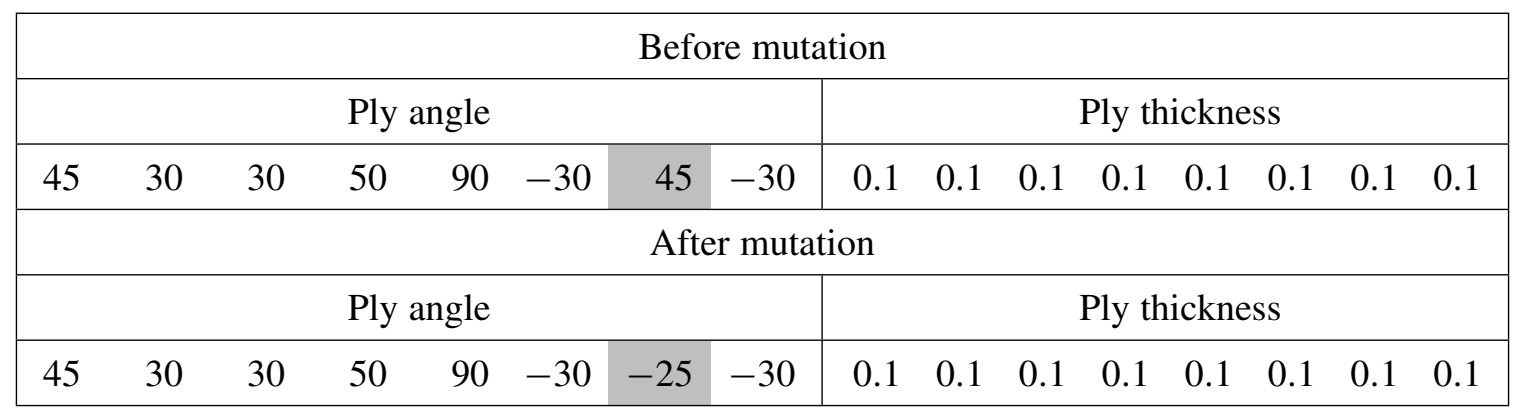

Table 5. An example of uniform mutation for NSGA-II.

\begin{tabular}{|c|c|c|c|c|c|c|c|c|c|c|c|c|c|c|c|}
\hline \multicolumn{16}{|c|}{ Before ply deletion } \\
\hline \multicolumn{8}{|c|}{ Ply angle } & \multicolumn{8}{|c|}{ Ply thickness } \\
\hline 45 & 30 & 30 & 50 & 90 & -30 & 45 & -30 & 0.1 & 0.1 & 0.1 & 0.1 & 0.1 & 0.1 & 0.1 & 0.1 \\
\hline \multicolumn{16}{|c|}{ After ply deletion } \\
\hline \multicolumn{8}{|c|}{ Ply angle } & \multicolumn{8}{|c|}{ Ply thickness } \\
\hline 45 & 30 & 30 & 50 & 90 & -30 & 45 & -30 & 0.1 & 0.1 & 0.1 & 0.1 & 0.1 & 0.1 & 0 & 0.1 \\
\hline
\end{tabular}

Table 6. An example of ply deletion operator for NSGA-II.

\section{Numerical results and discussion}

3.1. Validation of neural network. In general, the trained network performs well during the training session. However, there may be possibilities of over fitting when the same network is applied to the newly generated data. Hence, the trained network must be validated before it is included in the optimization procedure. The laminated composite plate made with the elliptical cut-out is considered, in which the 

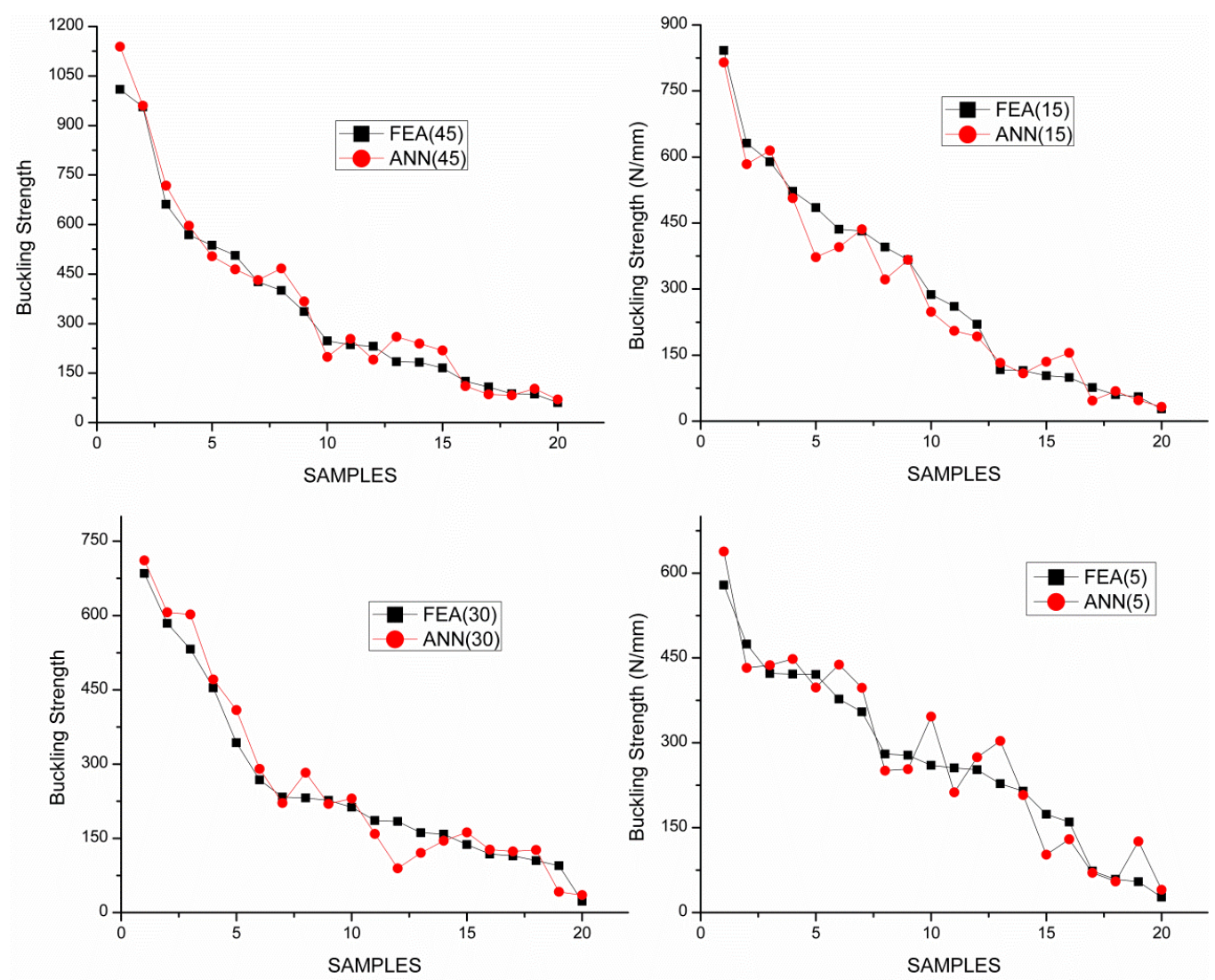

Figure 6. NN predictions versus actual targets for various ply angle intervals.

cutout is oriented at an angle $60^{\circ}$ and located at the center of the plate as shown in Figure 1. Twenty stacking sequences are generated for different ply angle intervals and the buckling strengths have been predicted using the trained networks as well as the finite element method. The results obtained using FEM and ANN have been compared in the Figure 6, which shows that the predictions of outputs are nearer to the actual targets in all the cases.

3.2. Multiobjective optimization. The ANN based NSGA-II is constructed and used to find the Pareto optimal solutions of the complex multiobjective optimization of the laminated composite plate with cutout. The maximum thickness of the plate is set as $3 \mathrm{~mm}$ whereas each ply has a thickness of $0.125 \mathrm{~mm}$. The number of plies, angle of each ply and stacking sequences are optimized for five different ply angle intervals $\left(45^{\circ}, 30^{\circ}, 15^{\circ}\right.$ and $\left.5^{\circ}\right)$. Hence, five different networks are constructed, trained and used for the multiobjective optimization problem. After the training, the networks are validated using new data. The performances of the networks during the testing period are shown in Figure 7 and it shows that the predictions of the networks are very close to the FEM results. Hence, the networks are recommended to be used in NSGA-II to predict the fitness values.

The Pareto optimal solutions obtained for different ply angle intervals using ANN based NSGA-II are shown in Figure 8. The results prove that the number of solutions in the Pareto optimal set is significantly 

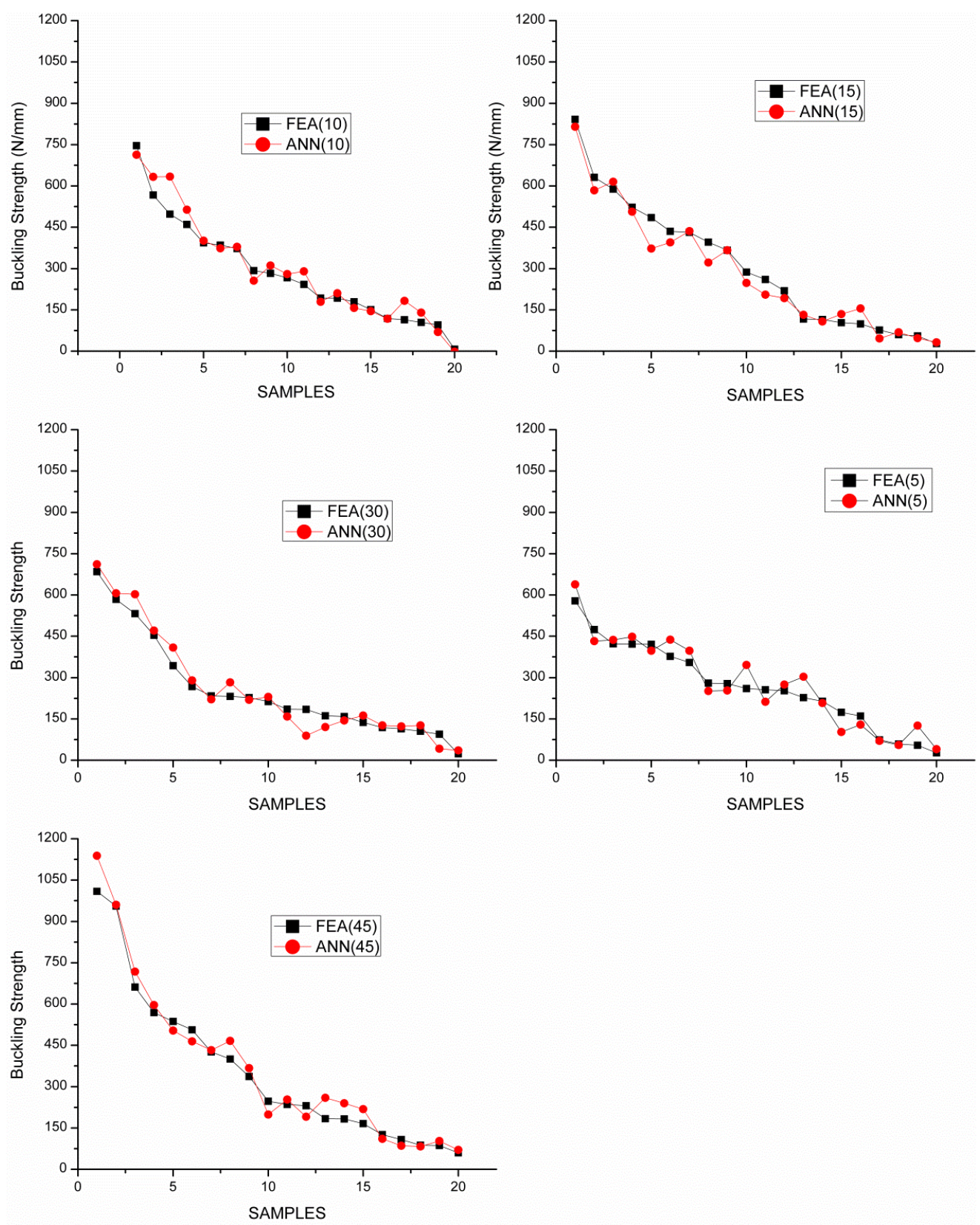

Figure 7. Performance of the networks during the testing.

increased by reducing the ply-angle interval. Only three solutions were found in the Pareto optimal set when conventional ply angles have been optimized. The number of solutions in the Pareto optimal set was increased from three to five when the ply angle interval has been reduced from $45^{\circ}$ to $30^{\circ}$. Further, it is found that the number of solutions was increased to six and twelve when the ply angle interval has 


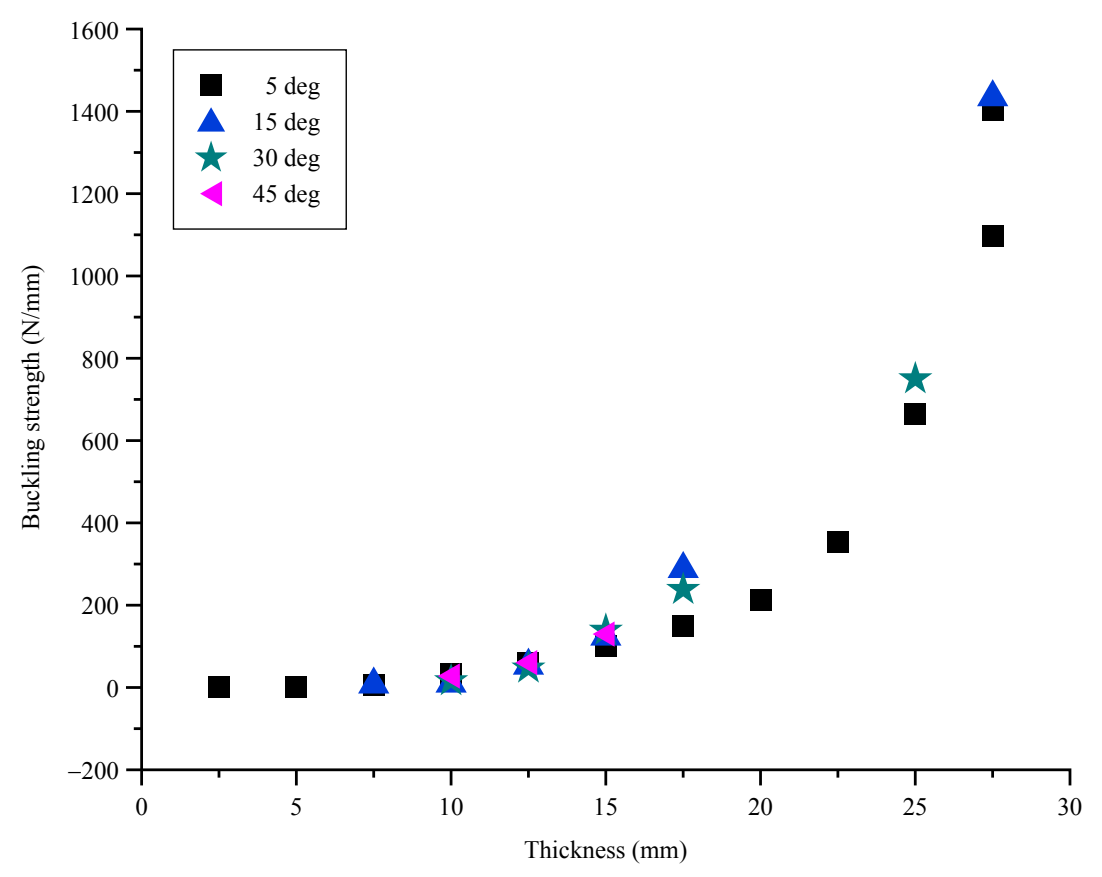

Figure 8. Pareto optimal solutions for the composite plate with cut-out.

been reduced to $15^{\circ}$ and $5^{\circ}$, respectively. The results also show that the boundaries of the Pareto optimal front are significantly increased by using the concept of ply angles having the reduced intervals.

Based on the trial and error method the maximum number of generations is set as 300 , whereas each generation works with 40 populations. If FEM had been used to analyze the plate, ABAQUS would have been called 12,000 times during the optimization process. As each run of FEM consumes approximately 18 seconds, more than sixty hours are required to complete the stacking sequence optimization problem. Further, GA is one of the stochastic optimization (SO) methods and hence, it has to be run several times in order to ensure for the global optima. This makes the FEM based optimization process more complex. However, the same optimization problem has been completed in less than 15 minutes using ANN based optimization method. Though the data generation task (for which FEM must be used) consumes about 5 hours, the weights and biases of the trained network are stored and used in future at any number of times in order to find the optimum GA control parameters and to ensure for global optima.

The number of Pareto optimal solutions obtained for various ply angle intervals are given in Table 7 and they are compared based on the objective values of the boundary solutions. It is found that only three Pareto optimal solutions were obtained when conventional ply angles have been optimized. The number of Pareto optimal solutions was increased from three to five by reducing the ply angle interval from $45^{\circ}$ to $30^{\circ}$. Further, the number of solutions in the Pareto optimal set was increased to six and twelve, when the ply angle interval has been reduced to $15^{\circ}$ and $5^{\circ}$, respectively. Thus, the concept of ply angles having the reduced intervals not only reduces the number of groupings of plies having the same orientation, but also increases the number of solutions in the Pareto optimal set as well as enhances 


\begin{tabular}{|c|c|c|c|c|c|c|c|}
\hline \multirow[t]{3}{*}{$\begin{array}{c}\text { Ply angle } \\
\text { interval (" } \Delta ")\end{array}$} & \multirow[t]{3}{*}{$\begin{array}{l}\text { Number of } \\
\text { solutions } \\
\text { in the Pareto } \\
\text { optimal set }\end{array}$} & \multicolumn{3}{|c|}{$\begin{array}{l}\text { Maximum buckling strength } \\
\text { and the corresponding } \\
\text { plate thickness }\end{array}$} & \multicolumn{3}{|c|}{$\begin{array}{l}\text { Maximum plate thickness } \\
\text { and the corresponding } \\
\text { buckling strength }\end{array}$} \\
\hline & & \multicolumn{2}{|c|}{ Buckling strength $^{\dagger}$} & \multirow[t]{2}{*}{ Thickness ${ }^{\dagger \dagger}$} & \multirow[t]{2}{*}{ Thickness $^{\dagger \dagger}$} & \multicolumn{2}{|c|}{ Buckling strength $^{\dagger}$} \\
\hline & & ANN & FEA & & & ANN & FEA \\
\hline $45^{\circ}$ & 3 & 28.29 & 27.88 & 10 & 15 & 130.55 & 134.7 \\
\hline $30^{\circ}$ & 5 & 16.36 & 16.14 & 10 & 25 & 750.23 & 738.45 \\
\hline $15^{\circ}$ & 6 & 6.856 & 7.05 & 7.5 & 27.5 & 1433.25 & 1485.2 \\
\hline $5^{\circ}$ & 12 & 1.2 & 1.17 & 2.5 & 27.5 & 1403.15 & 1359.52 \\
\hline
\end{tabular}

Table 7. Comparison of Pareto optimal solutions. Units are ${ }^{\dagger} \mathrm{N} / \mathrm{mm}$ and ${ }^{\dagger \dagger} \mathrm{mm}$.

the degree of the objective functions. In addition, the predictions of ANN are once again compared using FEA in Table 7, which proves that the performance of ANN is quite good even for the new data.

\section{Conclusions}

The Pareto optimal solutions for the laminated composite plate made with cut-out have been obtained using ANN based NSGA-II. The predictions of ANN have been found to be very close to the actual solutions. As weights and biases of the network can be stored, it is able to run NSGA several times to ensure the global optima. The number of solutions in the Pareto optimal set has been significantly increased by reducing the ply angle interval. The boundaries of the Pareto optimal set have been increased using the concept of reduced ply angle intervals. Accordingly, the degree of the objective functions has been enriched.

\section{References}

[Al-Assaf and El Kadi 2001] Y. Al-Assaf and H. El Kadi, "Fatigue life prediction of unidirectional glass fiber/epoxy composite laminae using neural networks", Compos. Struct. 53:1 (2001), 65-71.

[Al-Haik et al. 2006] M. S. Al-Haik, M. Y. Hussaini, and H. Garmestani, "Prediction of nonlinear viscoelastic behavior of polymeric composites using an artificial neural network”, Int. J. Plast. 22:7 (2006), 1367-1392.

[Al Qablan et al. 2009] H. Al Qablan, H. Katkhuda, and H. Dwairi, "Assessment of buckling behavior of square composite plates with circular cutout subjected to in-plane shear", Jordan J. Civ. Eng. 3:2 (2009), 184-195.

[Almeida and Awruch 2009] F. S. Almeida and A. M. Awruch, "Design optimization of composite laminated structures using genetic algorithms and finite element analysis", Compos. Struct. 88:3 (2009), 443-454.

[Aydin Komur et al. 2010] M. Aydin Komur, F. Sen, A. Atas, and N. Arslan, "Buckling analysis of laminated composite plates with an elliptical/circular cutout using FEM", Adv. Eng. Softw. 41:2 (2010), 161-164.

[Aymerich and Serra 2008] F. Aymerich and M. Serra, "Optimization of laminate stacking sequence for maximum buckling load using the ant colony optimization (ACO) metaheuristic", Compos. A Appl. Sci. Manuf. 39:2 (2008), 262-272.

[Bilgehan 2011] M. Bilgehan, "Comparison of ANFIS and NN models: with a study in critical buckling load estimation", Appl. Soft Comput. J. 11:4 (2011), 3779-3791.

[Bolanča et al. 2014] T. Bolanča, v. Ukić, I. Peternel, H. Kušić, and Božić, "Artificial neural network models for advanced oxidation of organics in water matrix-comparison of applied methodologies", Indian J. Chem. Tech. 21:1 (2014), 21-29. 
[Campbell 2010] F. C. Campbell, Structural composite materials, ASM International, Materials Park, OH, 2010.

[Cardozo et al. 2011] S. D. Cardozo, H. Gomes, and A. M. Awruch, "Optimization of laminated composite plates and shells using genetic algorithms, neural networks and finite elements”, Lat. Amer. J. Solids Struct. 8:4 (2011), 413-427.

[Chakraborty 2005] D. Chakraborty, "Artificial neural network based delamination prediction in laminated composites", Mater. Des. 26:1 (2005), 1-7.

[Deb 2001] K. Deb, Multi-objective optimization using evolutionary algorithms, Wiley, Chichester, 2001.

[Emmanuel Nicholas et al. 2014] P. Emmanuel Nicholas, K. P. Padmanaban, and D. Vasudevan, "Buckling optimization of laminated composite plate with elliptical cutout using ANN and GA", Struct. Eng. Mech. 52:4 (2014), 815-827.

[Erklig and Yeter 2012] A. Erklig and E. Yeter, "The effects of cutouts on buckling behavior of composite plates", Sci. Eng. Compos. Mater. 19:3 (2012), 323-330.

[Iyengar and Vyas 2011] N. G. R. Iyengar and N. Vyas, "Optimum design of laminated composite under axial compressive load”, Sadhana Acad. Proc. Eng. Sci. 36:1 (2011), 73-85.

[Karnik et al. 2008] S. R. Karnik, V. N. Gaitonde, J. C. Rubio, A. E. Correia, A. M. Abrão, and J. P. Davim, "Delamination analysis in high speed drilling of carbon fiber reinforced plastics (CFRP) using artificial neural network model", Mater. Des. 29:9 (2008), 1768-1776.

[Kermanshahi and Iwamiya 2002] B. Kermanshahi and H. Iwamiya, "Up to year 2020 load forecasting using neural nets", Int. J. of Elec. Power Energy Sys. 24:9 (2002), 789 - 797.

[Kim 2007] J.-S. Kim, "Development of a user-friendly expert system for composite laminate design", Compos. Struct. 79:1 (2007), 76-83.

[Kim et al. 2005] J.-S. Kim, N.-P. Kim, and S.-H. Han, "Optimal stiffness design of composite laminates for a train carbody by an expert system and enumeration method", Compos. Struct. 68:2 (2005), 147-156.

[Liu et al. 2006] Y. Liu, F. Jin, and Q. Li, "A strength-based multiple cutout optimization in composite plates using fixed grid finite element method", Compos. Struct. 73:4 (2006), 403-412.

[Lopes et al. 2010] C. S. Lopes, Z. Gürdal, and P. P. Camanho, "Tailoring for strength of composite steered-fibre panels with cutouts", Compos. A Appl. Sci. Manuf. 41:12 (2010), 1760-1767.

[Mishra et al. 2010] R. Mishra, J. Malik, I. Singh, and J. a. P. Davim, "Neural network approach for estimating the residual tensile strength after drilling in uni-directional glass fiber reinforced plastic laminates", Mater. Des. 31:6 (2010), 2790-2795.

[Omkar et al. 2009] S. N. Omkar, R. Khandelwal, T. V. S. Ananth, G. Narayana Naik, and S. Gopalakrishnan, "Quantum behaved particle swarm optimization (QPSO) for multi-objective design optimization of composite structures", Expert Syst. Appl. 36:8 (2009), 11312-11322.

[Omkar et al. 2011] S. N. Omkar, J. Senthilnath, R. Khandelwal, G. Narayana Naik, and S. Gopalakrishnan, "Artificial bee colony (ABC) for multi-objective design optimization of composite structures", Appl. Soft Comput. J. 11:1 (2011), 489-499.

[Reddy et al. 2013] B. S. Reddy, J. S. Kumar, and K. V. K. Reddy, "Prediction of deflection and stresses of laminated composite plate with an artificial neural network aid", Int. J. Appl. Sci. Eng. 11:4 (2013), 393-413.

[Rocha et al. 2014] I. B. C. M. Rocha, E. Parente, Jr., and A. M. C. Melo, "A hybrid shared/distributed memory parallel genetic algorithm for optimization of laminate composites", Compos. Struct. 107:1 (2014), 288-297.

[Tsao and Hocheng 2008] C. C. Tsao and H. Hocheng, "Evaluation of thrust force and surface roughness in drilling composite material using Taguchi analysis and neural network", J. Mater. Process. Technol. 203:1-3 (2008), 342-348.

[Yuen and Lam 2006] K.-V. Yuen and H.-F. Lam, "On the complexity of artificial neural networks for smart structures monitoring", Eng. Struct. 28:7 (2006), 977 - 984.

[Zheng et al. 2009] S.-J. Zheng, Z.-Q. Li, and H.-T. Wang, "Research on delamination monitoring for composite structures based on HHGA-WNN", Appl. Soft Comput. J. 9:3 (2009), 918-923.

Received 26 May 2015. Revised 30 Sep 2015. Accepted 5 Oct 2015.

P. EMMANUEL NichOlas: p.emmanuelnicholas@gmail.com

Mechanical Engineering, PSNA College of Engineering and Technology, Dindigul 624622, India 
M. C. LENIN BABU: mcbhabu@gmail.com

School of Mechanical \& Building Sciences, VIT University, Chennai 600127, India

A. SATHYA Sofia: sathyasofia@gmail.com

Computer Science Engineering, PSNA College of Engineering and Technology, Dindigul 624622, India 


\title{
ANALYTICAL ESTIMATES FOR THE LATERAL THRUST IN BOLTED STEEL BUCKLING-RESTRAINED BRACES
}

\author{
Guido Bregoli, Francesco Genna and Giovanni Metelli
}

\begin{abstract}
Analytical expressions are developed for the lateral thrust exerted by the core of a buckling-restrained brace (BRB), when, upon a prescribed axial loading history, it buckles, coming into contact with the external retaining structure. Account is taken of (i) the elastic stiffness of the containment case; (ii) the possible flattened shape of each buckled wave in the zones of contact with the containment profiles; and (iii) the increase in the lateral thrust due to loading-unloading in the presence of linear kinematic hardening. Comparisons with both experimental and numerical results confirm the effectiveness of the analytical predictions. The proposed calculation techniques for the lateral thrust might be of help in the design procedures of BRBs.
\end{abstract}

\section{Introduction}

The design of buckling-restrained braces (BRB) is becoming an increasingly important topic in civil engineering, both because BRBs have been recognized to be effective in dissipating energy, and because they appear to be quite useful for structural upgrade with minimal interventions. Nevertheless, the behavior of BRBs is not yet understood in full. For example, reliable formulas are still lacking with reference to the design of the containing structure, an element expected to sustain large lateral forces deriving from the contact with the buckled internal core; these lateral forces, if not properly accounted for, can lead to the collapse, local or global, of the whole structural element.

Little attention seems to have been devoted, so far, to the calculation of the lateral thrust in BRBs as consequence of a given loading history. Basic engineering approaches can be found in [Lin et al. 2012; Takeuchi et al. 2010; Wu et al. 2014; Chou and Chen 2010; Zhao et al. 2014]; in these works a capacity design approach was often taken, with the aim of estimating only the maximum possible thrust acting on the retaining profiles, independently on the actual loading history. Moreover, a standard Euler's relation between applied axial load and buckled wavelength was always assumed, which is incorrect in the present situation.

Other work is mainly theoretical, such as that of [Chai 1998] which, however, considers the case of large displacements, which entails complications that are unneeded in BRB applications, where the gap between core and containment is usually very small. A brief discussion of the relevance of this type of work to the case of BRBs can be found in [Genna and Bregoli 2014].

The present paper illustrates new results that exploit the theory developed in [Genna and Bregoli 2014], where only a generic, fully elastic problem of buckling with frictionless contact was studied, for a monotonic loading condition only. The new results presented hereafter hold for BRBs in the elasticplastic case, and account both for friction and, at least in part, for the effect of cyclic loading. The main

Keywords: buckling-restrained braces, elastic-plastic buckling, unilateral contact, lateral thrust, restraining member design. 
goal is an attempt at obtaining working design expressions, for the lateral thrust in bolted steel BRBs, that could be considered reliable in engineering terms, despite the uncertainty of several governing parameters, such as the existing imperfections as well as the friction and the material properties.

The results thus obtained will be compared with available experimental and numerical ones described in detail elsewhere [Bregoli et al. 2014; Bregoli 2014; Genna and Gelfi 2012a].

\section{Theoretical background}

The structural element herein considered is illustrated in Figure 1, which refers to a BRB made of two main steel components: an internal plate, or core, which is the energy-dissipating element, and an external containment structure, that can be made of steel profiles, bolted to each other and designed so as to leave a nominal gap $s$ between their internal surfaces and the external ones of the core.

The structural model of Figure 2 is adopted to describe the postbuckling mechanics of a steel-bolted BRB. In it, the central linear element represents the deformable core; the upper and lower linear elements represent the containment profiles, described as rigid surfaces free to translate only, and connected to

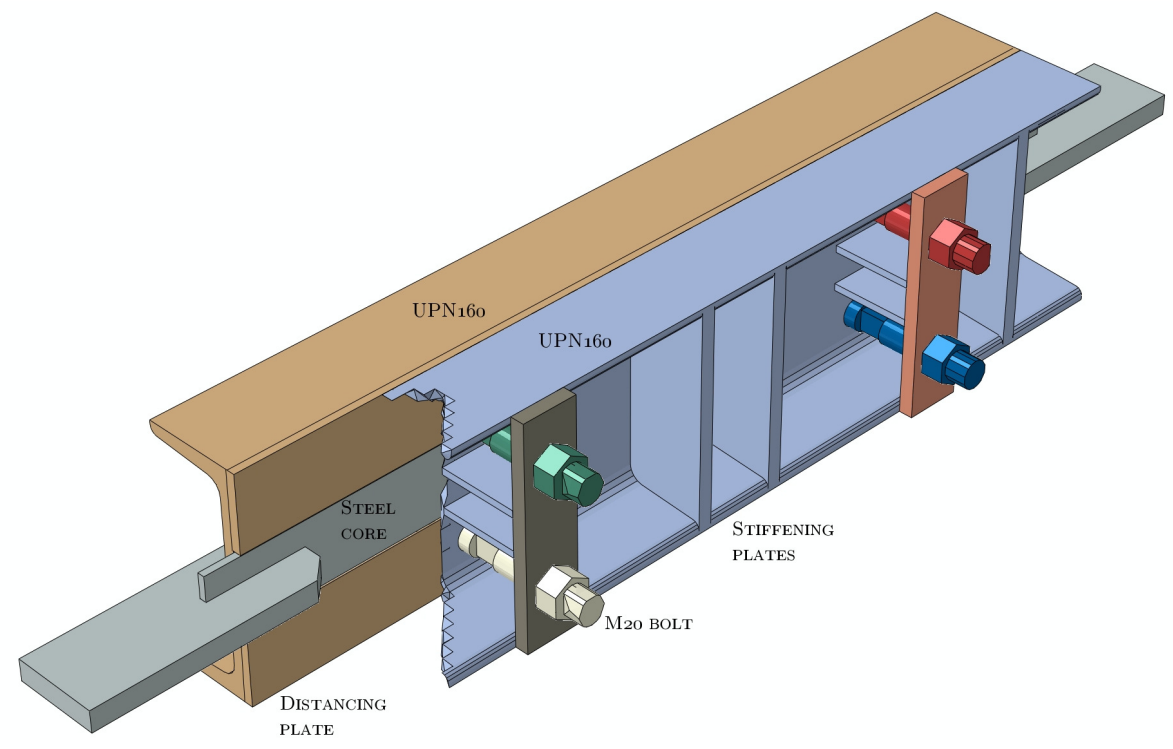

Figure 1. Sketch of the geometry of the studied BRB, showing the internal steel plate, the distancing plates, the two external C-shaped retaining steel profiles (one of them has been partially hidden), and the four connecting bolts. The transversal stiffening plates define an assembly called "design" in Section 6.

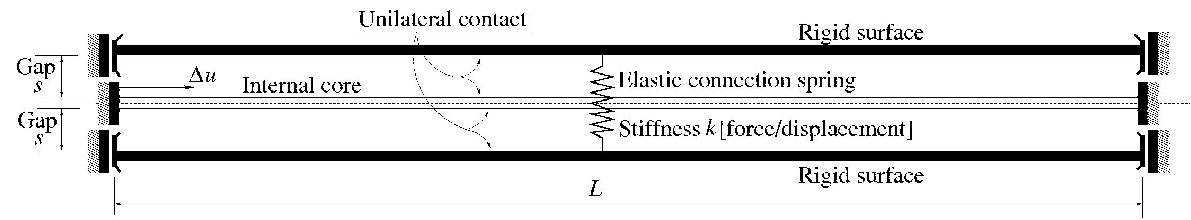

Figure 2. Analytical model for the study of a BRB assembly. 

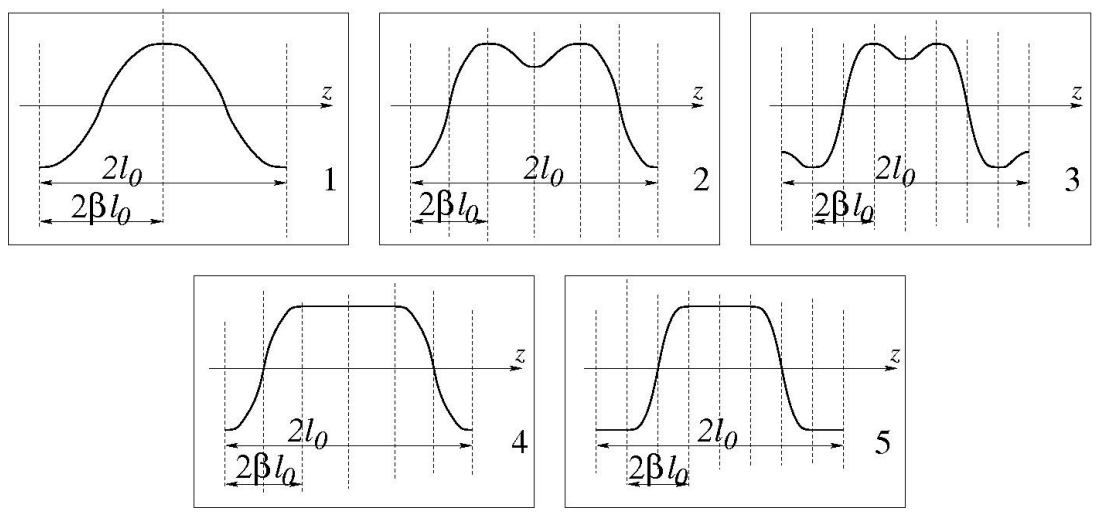

Figure 3. Buckled shapes (single waves shown): possible contact configurations considered in the analytical treatment. Image 1 is of a symmetric, single point contact. Image 2 is of an asymmetric point contact, which derives from the configuration of image 4 after local buckling of the flat top portion. Image 3 concerns a symmetric double point contact, deriving from the simultaneous local buckling of the flat portions in image 5 . Image 4 is of an asymmetric line contact configuration, with line contact on top and point contact on bottom. Image 5 is of a symmetric line contact configuration.

each other by means of a linear elastic spring, with elastic constant $k$ (dimension of force divided by displacement). This spring accounts for the actual deformability — both global and local — of the whole containment structure of Figure 1. Assuming always small values of the gap $s$ and small deflections, the analysis of this model is carried out in a second-order theory; shear strain contributions are neglected. It is assumed that the buckled configuration of the core is periodic, so that only half of a buckled wave needs to be studied. Throughout this paper, compression and shortening will be assumed as positive.

In order to consider the presence of line (flat) contacts between core and containment, it is assumed that the buckled shape can take any of the forms illustrated in Figure 3, which are of 3 types: (i) a pure point contact configuration, which has 3 possible subcases: with a single contact point at each side of each wave (image 1); or with one contact point at one side and two contact points at the other side of the core (image 2); or with two contact points at each side of each wave (image 3); (ii) an asymmetric line contact configuration (image 4), and (iii) a symmetric line contact configuration (image 5).

In the sequel of this work, $E$ denotes the Young modulus of the core, $A$ the area of its cross-section, $L$ its total length, $F$ the compressive axial force existing in the buckled core, related to the axial shortening $\Delta u$, in the elastic range, by the expression

$$
F=\frac{E A}{L} \Delta u
$$

Further, $I$ denotes the minimum moment of inertia of the core, $\alpha$ the ratio defined by

$$
\alpha^{2}=\frac{F}{E I}
$$




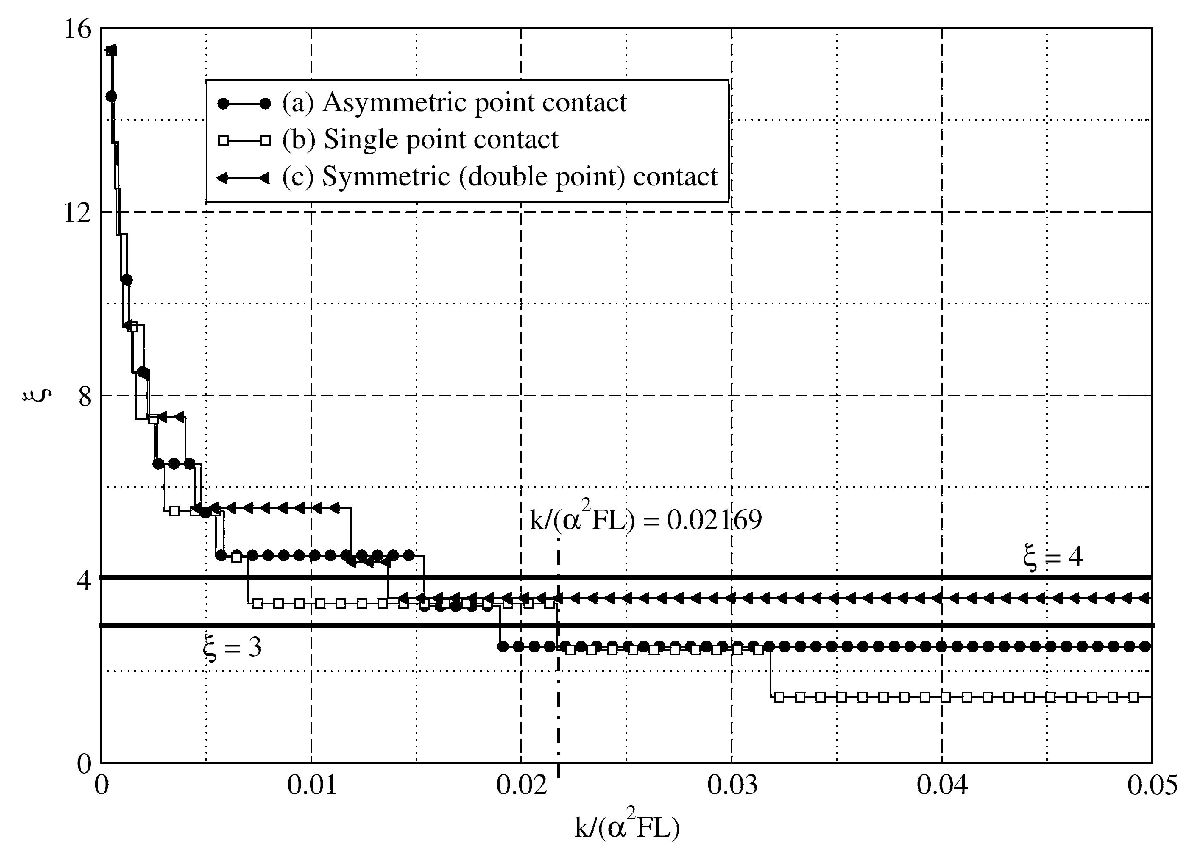

Figure 4. Possible values of $\xi$, defined in (4), as a function of the normalized spring stiffness $k /\left(\alpha^{2} F L\right)$. The horizontal solid lines describe maximum values for $\xi$ in the case of line contacts. The lines with symbols describe the possible values for $\xi$ deriving from situations of point contact only: black circles refer to asymmetric point contacts, as in Figure 3, image 4; white squares refer to single point contacts, as in Figure 3, image 1; black triangles refer to symmetric point contacts, as in Figure 3, image 5.

$N$ denotes the number of buckled waves, and $2 l_{0}$ the buckled wavelength. If the relationship between applied axial force and buckled wavelength obeyed a standard Euler theory, the following would hold:

$$
l_{0}=\frac{\pi}{\alpha}
$$

Euler's theory is not applicable to this case, however, because of the presence of contact forces between buckled core and external containment profiles.

Introducing a dimensionless parameter $\xi$ to define $l_{0}$ in a way similar to (3) as

$$
l_{0}=\xi \frac{\pi}{\alpha},
$$

the theory developed in [Genna and Bregoli 2014] leads to the results summarized hereafter, valid in the linear elastic case and for frictionless contact.

For the limiting case of $k \rightarrow \infty$, i.e., infinitely rigid retention profiles, $1.4303 \leq \xi \leq 4$ was the range of values that could be obtained for $\xi$ of (4), depending on the buckled configuration assumed by the core.

For finite values of $k$, the dependency shown in Figure 4 can be computed numerically for the three possible cases of a point contact. In Figure 4, curve (a) refers to the case of an asymmetric point contact, 
as in Figure 3, image 2; curve (b) refers to a pure point contact, as in Figure 3, image 1; and curve (c) refers to the case of a symmetric double point contact at each side of the beam, as in Figure 3, image 3 . It can be seen that, if only a point contact situation develops, the value of $\xi$ can become very high for small enough values of the stiffness $k$.

In the presence of a line contact, for any value of the stiffness $k$, it is proved that $\xi=3$ in the case of an asymmetric line contact, and $\xi=4$ in the case of a symmetric line contact.

The actual value of $\xi$, given a value of the axial shortening, is strongly dependent on the shape of the buckled configuration which, in turn, depends on several factors, among which the imperfections existing in a real structure. Therefore, the actual buckled configuration is theoretically unpredictable, since there are several possible alternatives among which to choose.

Given a buckled configuration, the corresponding expression for the lateral thrust $Q$ can be also computed; the following general result was derived in [Genna and Bregoli 2014], which gives the unit thrust $Q_{i}$, i.e., the contact force existing at each side of a single buckled wave:

$$
Q_{i}=\frac{4 \pi \xi \alpha k F s \cos (\pi \xi \beta)}{\cos (\pi \xi \beta)\left(2 \pi^{2} \xi^{2} \beta k-\alpha^{2} F L\right)-2 \pi \xi k \sin (\pi \xi \beta)},
$$

where $\beta$ is a parameter that identifies the length of the inclined portions of the wave between two successive contact points, as shown in Figure 3.

Both in the limiting case $k \rightarrow \infty$, and for all the buckled configurations with incipient or fully formed line contacts, with $\beta=1 / \xi$ as shown in [Genna and Bregoli 2014], (5) reduces to the trivial one

$$
Q_{i, \infty}=\frac{2 F \alpha s}{\pi} .
$$

In all these expressions, in the presence of a linear elastic material for the core, the value of $F$ is given by (1), and the value of $\alpha$ of (2) is

$$
\alpha=\frac{1}{\rho} \sqrt{\frac{\Delta u}{L}},
$$

where $\rho$ is the radius of inertia corresponding to $I$, i.e., $\rho^{2}=I / A$.

The total lateral thrust $Q$ is obtained by multiplying $Q_{i}$ for the number $N$ of waves in the buckled shape, i.e.,

$$
Q=Q_{i} N,
$$

with $N$ an integer number. Genna and Bregoli [2014] propose to adopt the following expressions for $\xi$ and $N$, assuming that the containment profiles are stiff enough to allow the formation of line contacts:

$$
l_{0}=\frac{3 \pi}{\alpha}, \quad N=\operatorname{Int}\left(\frac{L}{2 l_{0}}+0.5\right),
$$

where Int denotes the integer part. This result, and specifically the choice $\xi=3$, defines an axial force nine times larger than the normally adopted Euler one corresponding to a given wavelength $2 l_{0}$ in the linear elastic range.

The analysis in [Genna and Bregoli 2014] concludes with the observation that an acceptable engineering choice for the value of parameter $\xi$, even in the elastic-plastic range, remains $\xi=3$, as indicated in (9). In the next two sections, therefore, we will adopt as the best compromise for the value of $\xi$ the value 

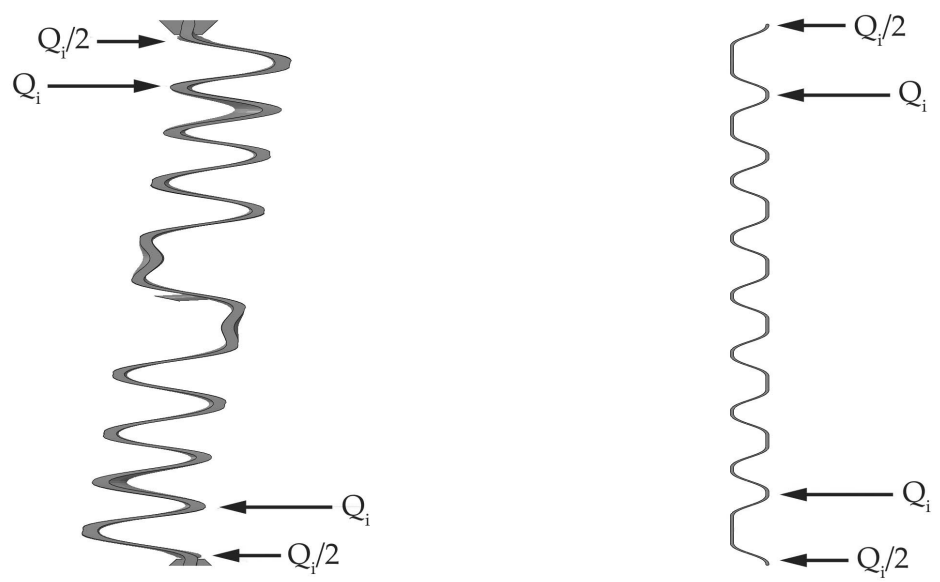

Figure 5. Buckled shapes in two different situations. Left image (from a 3D analysis with ABAQUS): the external containment can undergo rigid rotations. The number of contact points is the same at each side, and the total number of waves is not an integer (8.5, in this image). Right image (from a 2D analysis with ABAQUS in which the containment is described as in Figure 2): the external containment can not rotate. The number of contact points at one side (right, in the figure) is higher by one than at the opposite side; the number of waves is integer ( 9 , in this image).

$\xi=3$, corresponding to a configuration of asymmetric line contact, and therefore to expression (9) for the wavelength, and to expression (5) for the unit thrust, with $\beta=1 / \xi$. This implies that the containment profiles are stiff enough to enable the development of line contacts. Only Section 5 will discuss possible results deriving from different situations.

In the following, the calculation of the number $N$ of waves associated to $l_{0}$ is modified with respect to the result given by (9) proposed in [Genna and Bregoli 2014]. In fact, the examination of both experimental and numerical results (see [Bregoli et al. 2014; Bregoli 2014; Genna and Gelfi 2012a]) shows that it is possible to observe a fractional number of waves, associated to a configuration in which both sides of the core have the same number of contact points. In this case, the number of unit contact forces $Q_{i}$, at each side of the core, is of the type $I+0.5$, with $I$ an integer number, as shown in Figure 5, left image.

This situation derives from a rigid rotation of the external containment case, often possible in real applications but ruled out in the analytical model of [Genna and Bregoli 2014]. This latter must always show an integer number of waves, as in Figure 5, right image, whereas, if a rigid rotation occurs, the configuration can become that of Figure 5, left picture, and the number of waves can be fractional, with a possible difference of \pm half wave with respect to the integer $N$ case. In order to account for this possibility, the suggested expression for $N$, once computed $l_{0}$, is then

$$
N=\frac{1}{2} \operatorname{Int}\left(\frac{L}{l_{0}}+0.5\right)
$$

which yields a value for $N$ rounded to the closest 0.5 . 


\section{Extension to linear kinematic hardening and cyclic loading}

3.1. Linear hardening, monotonic loading. The material parameters describing the plastic properties are now a yield stress of the virgin material, $\sigma_{0}$, and either a tangent modulus $E_{t}$, i.e., the slope of the hardening branch in a uniaxial stress-strain curve, or, equivalently, a hardening coefficient $h$, i.e., the slope of the hardening branch in a uniaxial stress-plastic strain curve. These parameters are related to each other by the following relationships:

$$
E_{t}=\frac{h E}{E+h}, \quad h=\frac{E E_{t}}{E-E_{t}} .
$$

In practical BRB applications, the core can undergo only small deflections, since the gap $s$ is assumed to be small. It is then appropriate to consider that, in the plastic range, buckling occurs under increasing axial loading, i.e., with no local unloading at any point of any cross-section of the beam. In this case, Shanley's theory [Shanley 1947] of plastic buckling applies, which, for an Eulerian problem, would imply to replace, in the expression of the buckling load, the Young modulus $E$ with the tangent modulus $E_{t}$.

The problem under study is not Eulerian, but Shanley's theory holds also in this case, since the phenomena involved can be seen as a sequence of local Eulerian bucklings in the plastic range. Therefore, in the presence of an elastic-plastic linear hardening material (9) must be modified to

$$
l_{0}=3 \pi \sqrt{\frac{E_{t} I}{F}},
$$

where the correct expression for $F$, assigned a value $\Delta u$ of the axial shortening, should be adopted, according to the material behavior. Under the assumption of monotonic loading from zero up to an axial shortening $\Delta u$, this is done by writing

$$
F=A \sigma
$$

and

$$
\sigma=E_{t}\left(\bar{\varepsilon}+\frac{\sigma_{0}}{h}\right)
$$

where

$$
\bar{\varepsilon}=\frac{\Delta u}{L} .
$$

Finally, substituting (13) and (14) into (12), one obtains the desired result:

$$
l_{0}=\frac{3 \pi \rho}{\sqrt{\bar{\varepsilon}+\frac{\sigma_{0}}{h}}}
$$

Result (16) reduces to (9) in the case of linear elasticity $(h \rightarrow \infty)$, and yields $l_{0}=0$, i.e., an infinite number of buckled waves, for the case of perfect plasticity $(h=0)$, when, under a compressive axial load at the limit of the carrying capacity of the core, the stiffness vanishes.

In order to obtain the corresponding result for the lateral thrust, one must abandon expression (5), which derives from the linear elastic analysis summarized in Section 2. Recalling that the value $\xi=3$, in expression (9) of $l_{0}$, derives from a situation of line contact, one has the supplementary information that 


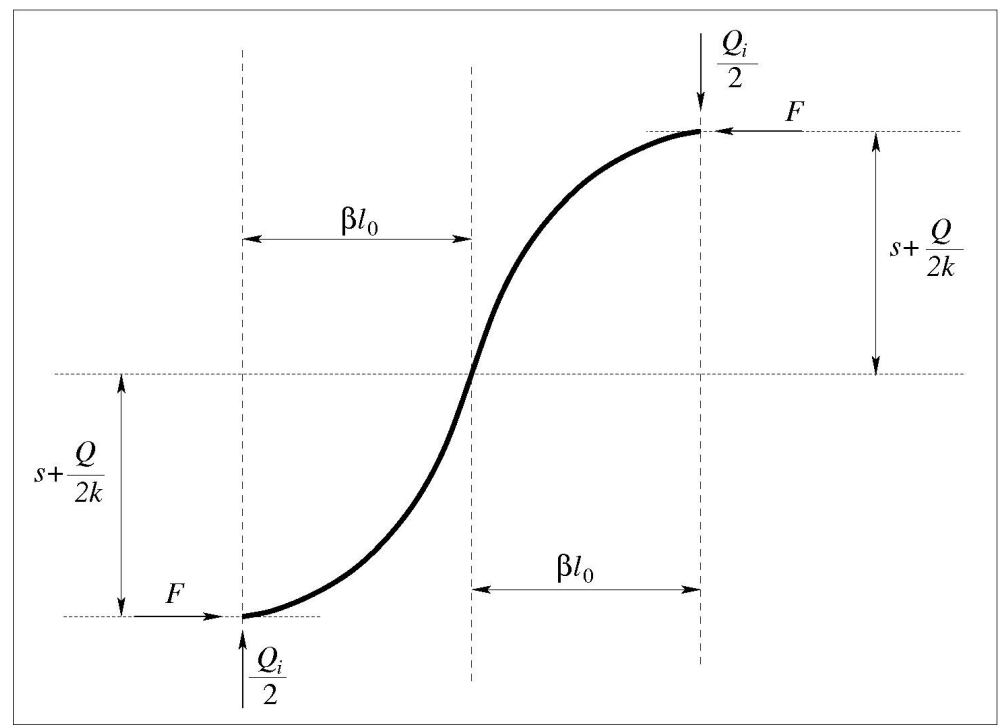

Figure 6. Deformed shape for the calculation of the unit thrust $Q_{i}$ in all the cases when the bending moment at the contact point vanishes.

the bending moment, at the contact points at both sides of the core, is zero. Therefore, one can compute the unit thrust $Q_{i}$ directly from equilibrium, as shown in Figure 6, thus obtaining

$$
Q_{i}=\frac{2 F s k}{\beta k l_{0}-F N},
$$

which, in the limit $k \rightarrow \infty$, reduces to

$$
Q_{i, \infty}=\frac{2 F s}{\beta l_{0}} .
$$

Setting $\xi=3$ and $\beta=1 / 3$ in (17), using (9) for $N$ and (16) for $l_{0}$, one obtains the desired result for the current situation.

3.2. Linear kinematic hardening, cyclic loading. In expression (16) for the buckled wavelength, the driving stress was defined through (14), which holds for monotonic loading only, from zero up to the prescribed axial displacement value.

In the case of cyclic loading, such expression does not hold anymore. One must in fact consider that, after a tension cycle that develops sufficiently large plastic strains, such as is expected to occur in real BRB applications, the core, during the next displacement reversal, and owing to irreversibility, starts being subjected to a compressive axial force while it is still elongated. Buckling is therefore associated to a different starting configuration than in the case of monotonic compressive loading.

This can be accounted for by redefining the current stress, at the end of a compressive loading cycle, as if it were reached following a fictitious monotonic loading condition.

Figure 7 illustrates the problem in terms of a stress-plastic strain diagram. In this plot, where compression and shortening are assumed positive, a loading cycle for a linear kinematic hardening material is considered, as produced by an axial displacement cycle between limits $-\Delta u_{t}$ and $\Delta u_{c}$ (subscripts $t$ 


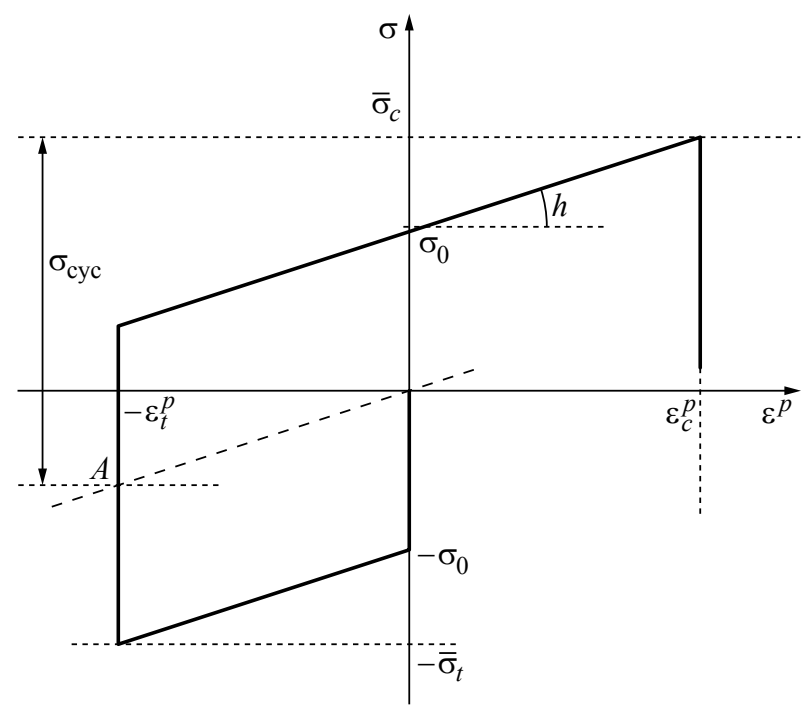

Figure 7. Stress-plastic strain loading cycle with linear kinematic hardening. Definition of $\sigma_{\text {cyc }}$ to be adopted for the calculation of the buckling load in the plastic range under cyclic loading.

and $c$ stand for tension and compression respectively), which correspond to strains $-\bar{\varepsilon}_{t}$ and $\bar{\varepsilon}_{c}$, and to plastic strains $-\varepsilon_{t}^{p}$ and $\varepsilon_{c}^{p}$, respectively. The actual stress values corresponding to the cycle strain limits $\bar{\varepsilon}_{c}$ and $-\bar{\varepsilon}_{t}$ would be $\bar{\sigma}_{c}$ and $-\bar{\sigma}_{t}$ respectively, both given by an equation of the type of (14).

Expression (16) for $l_{0}$, derived for monotonic loading, can be extended to the case in which the BRB core has undergone the nonmonotonic stress-strain history indicated in Figure 7, that can also include an arbitrary number of previous cycles with the same limits. To do so, a modified stress value must be inserted into equations (13) and (16), replacing the actual stress $\sigma$ with a fictitious value $\sigma_{\text {cyc }}$. The stress $\sigma_{\text {cyc }}$ is defined as if the current plastic strain amplitude $\varepsilon_{c}^{p}$ in compression had been reached monotonically, but starting from point A in Figure 7, after the elastic deformation recovery of the first semicycle in tension.

This leads to the following calculation:

$$
\sigma_{\mathrm{cyc}}=\sigma_{0}+h \varepsilon_{c}^{p}+h \varepsilon_{t}^{p}=\sigma_{0}+h\left(\bar{\varepsilon}_{c}-\bar{\varepsilon}_{c}^{e}+\bar{\varepsilon}_{t}-\bar{\varepsilon}_{t}^{e}\right)=\sigma_{0}+h\left(\bar{\varepsilon}_{c}-\frac{\bar{\sigma}_{c}}{E}+\bar{\varepsilon}_{t}-\frac{\bar{\sigma}_{t}}{E}\right) .
$$

Adopting for $\bar{\sigma}_{c}$ and $\bar{\sigma}_{t}$ the expression of (14), one obtains

$$
\sigma_{\mathrm{cyc}}=E_{t}\left(\bar{\varepsilon}_{c}+\bar{\varepsilon}_{t}+\frac{\sigma_{0}(E-h)}{E h}\right) .
$$

The insertion of result (20) into (16) would allow one to obtain an expression for $l_{0}$ holding for any type of cycle. Nevertheless, in the present work we will consider only the special case of cycles of equal amplitude in tension and compression, i.e., $\bar{\varepsilon}_{c}=\bar{\varepsilon}_{t}=\bar{\varepsilon}$. This will be done because all the results presented in the next sections will refer to the experimental cycles prescribed by the AISC regulations [AISC 2010], which have equal amplitude in tension and in compression. In such case, it can be immediately seen that 
(20) implies a driving strain twice the one holding for monotonic loading:

$$
\sigma_{\mathrm{cyc}}=E_{t}\left(2 \bar{\varepsilon}+\frac{\sigma_{0}(E-h)}{E h}\right) .
$$

Restarting from (12) and (13), and replacing expression (21) for the stress into (13), one obtains what follows for the semiwavelength:

$$
l_{0}=3 \pi \rho \sqrt{\frac{h E}{2 \bar{\varepsilon} h E+\sigma_{0}(E-h)}} .
$$

This result must be inserted into (17) for the unit thrust. Nevertheless, since (17) derives just from equilibrium, the expression of the axial force $F$, in it, should remain the same as in the case of monotonic loading, i.e., (13) with stress $\sigma$ given by (14).

\section{Extension to contact with friction}

Coulomb friction is assumed, with friction coefficient $\mu$; typical values, for BRB applications, are $\mu=0.15$ for lubricated steel surfaces, and $\mu=0.25$ for unlubricated steel surfaces, as measured, for instance, in [Genna and Gelfi 2012a].

The correction for including friction amounts to increasing the driving axial compressive force $F$, associated to the prescribed axial shortening $\Delta u$, by the quantity

$$
\Delta F=\frac{\mu Q_{0}}{2},
$$

where $Q_{0}$ denotes the total thrust of (8), with $Q_{i}$ as in (17), computed in the absence of friction, with $F$ associated to the real stress of (14), and $l_{0}$ given by (22). This is only a first approximation, which, however, appears to be effective from an engineering viewpoint.

As a consequence, the result for the wavelength should be recomputed replacing, in it, the value of $F$ with $F+\Delta F$. Recalling (12), the final expression for $l_{0}$ is thus

$$
l_{0}=\frac{3 \pi \sqrt{E_{t} I}}{\sqrt{\sigma_{\mathrm{cyc}} A+\mu Q_{0} / 2}} .
$$

The same correction to the axial force $F$ must be applied to the formulas for the subsequent calculation of the unit and total thrust, recalling that, in these calculations, the real value of the axial force associated to $\bar{\sigma}$, and increased by friction, should be considered.

We finally summarize the actual procedure to be followed to estimate the total thrust in a real BRB subjected to cyclic loading, variable between equal positive and negative limits (a more general result could be easily obtained adopting (20) for the corrected axial stress at the end of a nonsymmetric loading cycle).

(1) Compute a first estimate of the wavelength with no friction from (22).

(2) From this, using (10), compute $N_{0}$, the rounded number of waves associated to the estimated wavelength. 
(3) Recompute a corrected wavelength as

$$
l_{0}^{*}=\frac{L}{2 N_{0}}
$$

associated to the rounded number of waves.

(4) Recompute corrected values $\xi^{*}$ and $\beta^{*}=1 / \xi^{*}$ from $l_{0}^{*}$, associated to the rounded number of waves. This is done on the basis of definition (4), to be now rewritten in the elastic-plastic, cyclic loading context, i.e., as

$$
l_{0}=\xi \pi \sqrt{\frac{E_{t} I}{\sigma_{\mathrm{cyc}} A+\mu Q_{0} / 2}},
$$

with $Q_{0}=0$ for the time being.

(5) Compute $Q_{0}$ from (17) and (8). Recall that, so far, all these quantities hold for the frictionless case; recall also that, in the calculation of the lateral thrust, the expression for the monotonic stress (14) should be adopted when computing the axial force $F$.

(6) Compute a first estimate of the actual wavelength, with friction, from (24).

(7) Compute the correct rounded number of waves, $N$, using (10).

(8) Compute the correct wavelength as

$$
l_{0}=\frac{L}{2 N}
$$

associated to the rounded number of waves $N$.

(9) Compute the correct values for $\xi$ and $\beta=1 / \xi$ from $l_{0}$, associated to the rounded number of waves $N$, using (26).

(10) Compute $Q_{i}$ from (17) and $Q$ from (8), with $F$ associated to the monotonic stress of (14) and increased to consider friction, using the rounded number of waves $N$.

An analysis of (17), using also (27), shows that the total thrust $Q$ depends on the ratio $s b / t$, where $b$ is the width and $t$ the height of the core cross-section.

\section{Small stiffness of the containment structure}

The containment structure, in real BRB applications, has a finite elastic stiffness that derives from the presence of both the connection bolts and the global/local deformability of the profiles employed as a retaining case. Such a stiffness can be taken into account, in the expressions proposed above, by suitably computing the numerical value of the coefficient $k$, i.e., the elastic stiffness of the connection spring in Figure 2.

Genna and Bregoli [2014] suggest to always use the results holding in the limit $k \rightarrow \infty$, in order to stay clear of the complex behaviors deriving from the use of finite values for $k$. This implies a corresponding attention in designing a sufficiently stiff containment structure. In fact, if a sufficient stiffness can not be guaranteed, and even ruling out a situation of possible local plastic collapse of the containment profiles, the situation becomes complicated: 
- Given a value of $\Delta u$, a reduction of $k$, assuming that the number of buckled waves remains the same, implies an increase of the total thrust, which, in theory, could reach an infinite value.

- Given a value of $\Delta u$, a reduction of $k$ might cause a decrease of the number of buckled waves, as shown in Figure 4, and as is intuitive, considering that for $k=0$ only one wave should appear; if the number of waves is smaller, a smaller thrust is expected.

- Nevertheless, if a change in the number of buckled waves is also associated to a change of the buckled configuration, then it is possible that an opposite effect happens. Consider the curves of Figure 4, for example in the case of a symmetric double point contact configuration: for $k /\left(\alpha^{2} F L\right)=$ 0.03 the predicted value for $\xi$ would be $\xi \approx 3.586287$. If a reduction of the value of $k$ to $k /\left(\alpha^{2} F L\right)=$ 0.01 is considered, then the corresponding value for $\xi$, for the same configuration, would be $\xi \approx$ 5.548147, with a smaller number of waves and a smaller thrust to be expected, according to intuition. But - and here lies the complication - if the value of $k$ becomes small enough, then the occurrence of a double point contact becomes less and less likely, since the confinement structure does not offer enough support for this phenomenon, which can only descend from a previous line contact situation. Therefore, it might well happen that, for $k /\left(\alpha^{2} F L\right)=0.01$, a single point contact configuration occurs, for the given value of $\Delta u$, to which the value $\xi \approx 3.47089$ corresponds: a number of waves higher even than for the symmetric point contact configuration with $k /\left(\alpha^{2} F L\right)=0.03$, and a higher thrust, contrary to the expectations, in the presence of a reduced value for $k$.

In view of these observations, the following procedure is suggested, for the choice of the appropriate value for $\xi$ in the presence of a given finite value for $k$ : choose the value of $\xi$ from the single point contact curve of Figure 4 (the lowest of the three, with white square symbols), but use always $\xi \geq 3$. This choice seems reasonable because, as said, for small values of $k$ line contacts are less and less expected. According to this criterion, the set of values for $\xi$ shown in Table 1 should be adopted, assuming that even for very small values of $k$ the assumptions underlying the theory hold.

\begin{tabular}{|l|c|}
\hline \multicolumn{1}{|c|}{ Stiffness $k$ range } & $\xi$ \\
\hline $0.00055<k /\left(\alpha^{2} F L\right) \leq 0.000683$ & 13.49249 \\
$0.000683<k /\left(\alpha^{2} F L\right) \leq 0.000725$ & 12.49189 \\
$0.000725<k /\left(\alpha^{2} F L\right) \leq 0.000977$ & 11.49118 \\
$0.000977<k /\left(\alpha^{2} F L\right) \leq 0.00105$ & 10.49034 \\
$0.00105<k /\left(\alpha^{2} F L\right) \leq 0.00151$ & 9.489326 \\
$0.00151<k /\left(\alpha^{2} F L\right) \leq 0.00165$ & 8.488068 \\
$0.00165<k /\left(\alpha^{2} F L\right) \leq 0.00265$ & 7.486474 \\
$0.00265<k /\left(\alpha^{2} F L\right) \leq 0.003$ & 6.484387 \\
$0.003<</\left(\alpha^{2} F L\right) \leq 0.00583$ & 5.481536 \\
$0.00583<k /\left(\alpha^{2} F L\right) \leq 0.007$ & 4.477408 \\
$0.007<k /\left(\alpha^{2} F L\right) \leq 0.02169$ & 3.47089 \\
$0.02169<k /\left(\alpha^{2} F L\right)$ & 3 \\
\hline
\end{tabular}

Table 1. Values of the coefficient $\xi$ as a function of the normalized value of the spring stiffness $k$. 
In practice, values $\xi>5$ should not be expected since, in the case of such small values of the stiffness $k$, a BRB would hardly behave as it should. In practice, again, all this suggests that the stiffness of the containment structure, given a maximum value of the design axial force, should preferably be not less than the limit value $k_{\lim }$ defined by

$$
k \geq k_{\lim }, \quad k_{\lim } \approx 0.022 \alpha^{2} F L
$$

in order to use the "safe" results of the previous section and, at the same time, guarantee for the correct functioning of the BRB.

If use is made of a stiffness-corrected value for $\xi$ from Table 1, if $\xi>3$ then a point contact configuration is expected, and the value of parameter $\beta$, for the calculation of the unit thrust $Q_{i}$, must be changed accordingly, since $\beta=1 / \xi$ is valid only for a situation of line contact. The value associated to a single point contact situation is $\beta=0.5$, as shown in [Genna and Bregoli 2014], and obvious from Figure 3 . Apart from the possible redefinition of $\xi$ and $\beta$, everything else remains unchanged in the procedure illustrated at the end of the previous section. Even the calculation of $Q_{i}$ from (17) remains still valid because, in the fully plastic range, the bending moment, at the points of contact, is expected to be always negligible.

Appendix A illustrates an example of how to compute the stiffness $k$ for a specific arrangement of the containment profiles.

\section{Comparison with experimental and numerical results}

The proposed analytical procedure has been tested for the reduced-scale BRBs studied in [Bregoli et al. 2014; Bregoli 2014; Genna and Gelfi 2012a]. These comprise a steel core plate enclosed into 2 bolted C-shaped steel struts, that act as a retaining case, made of two UPN160 commercial profiles, as shown in Figure 1. Eight different BRB assemblies have been tested: in five of them the steel core has a $50 \times 5 \mathrm{~mm}$ cross-section, and in the remaining three the core has a $50 \times 7 \mathrm{~mm}$ cross-section. The length of the core is $L=560 \mathrm{~mm}$. Five different core-case gap values have been considered: $2 \times 0.25,2 \times 0.46,2 \times 0.5$, $2 \times 0.70$, and $2 \times 1 \mathrm{~mm}$, i.e., $s=0.25,0.46,0.5,0.7$, and $1 \mathrm{~mm}$ at each side of the core. In the sequel, the different specimens will be denoted, according to the core thickness $t$ and the nominal gap size $s$, by $5+0.25,5+0.46,5+0.5,5+0.7,5+1,7+0.25,7+0.5$, and $7+1$. For all the details of these samples, see [Bregoli et al. 2014; Bregoli 2014; Genna and Gelfi 2012a].

The experimental results have been obtained following the AISC prescriptions for the cyclic loading type [AISC 2010]. The final step has a value $\Delta u= \pm 11.2 \mathrm{~mm}$, so as to have, at the end of the last two cycles, $\bar{\varepsilon}= \pm 0.02$ in the core.

Three different arrangements of the retaining profiles have been considered. The basic one, denoted in the following by "design", makes use of two UPN160 profiles with transversal stiffening ribs welded to them (see Figure 1). A stiffer geometry has also been tested, denoted by "rigid" in the following, in which an additional longitudinal plate has been welded in the center of the UPN web, along its main axis; the reinforcing plate was doubled, and welded at the thirds of the web length, in the central zone of the assembly. In other cases, also a more deformable assembly was tested, in which no stiffening rib at all was welded to the UPN160 profiles. This setup will be denoted as "Deformable" in the following. 


\begin{tabular}{|c|lcccccccc|}
\hline \multirow{3}{*}{$k[\mathrm{~N} / \mathrm{mm}]$} & BRB type & $5+0.25$ & $5+0.46$ & $5+0.5$ & $5+0.7$ & $5+1$ & $7+0.25$ & $7+0.5$ & $7+1$ \\
\hline & "Deformable" & 340708 & 340616 & 340598 & 340510 & 340379 & 324224 & 324124 & 323925 \\
& "Design" & 551968 & 551725 & 551679 & 551449 & 551103 & 527214 & 526951 & 526425 \\
& "Rigid" & 671904 & 671545 & 671477 & 671136 & 670624 & 643129 & 642737 & 641955 \\
\hline \multirow{5}{*}{$F[\mathrm{kN}]$} & "Deformable" & 102.6 & 104.8 & 105.2 & 107.3 & 110.5 & 141.5 & 143.2 & 147.2 \\
& "Design" & 102.4 & 104.4 & 104.8 & 106.8 & 109.7 & 141.5 & 143.0 & 146.8 \\
& "Rigid" & 102.3 & 104.3 & 104.7 & 106.6 & 109.5 & 141.4 & 143.0 & 146.7 \\
& $k \rightarrow \infty$ & 102.1 & 103.9 & 104.3 & 106.0 & 108.7 & 141.3 & 142.8 & 146.3 \\
\hline \multirow{5}{*}{$l_{0}[\mathrm{~mm}]$} & "Deformable" & 37.33 & 37.33 & 37.33 & 37.33 & 37.33 & 56 & 56 & 50.91 \\
& "Design" & 37.33 & 37.33 & 37.33 & 37.33 & 37.33 & 56 & 56 & 50.91 \\
& "Rigid" & 37.33 & 37.33 & 37.33 & 37.33 & 37.33 & 56 & 56 & 50.91 \\
& $k \rightarrow \infty$ & 37.33 & 37.33 & 37.33 & 37.33 & 37.33 & 56 & 56 & 50.91 \\
\hline \multirow{5}{*}{$N$} & "Deformable" & 7.5 & 7.5 & 7.5 & 7.5 & 7.5 & 5 & 5 & 5.5 \\
& "Design" & 7.5 & 7.5 & 7.5 & 7.5 & 7.5 & 5 & 5 & 5.5 \\
& "Rigid" & 7.5 & 7.5 & 7.5 & 7.5 & 7.5 & 5 & 5 & 5.5 \\
& $k \rightarrow \infty$ & 7.5 & 7.5 & 7.5 & 7.5 & 7.5 & 5 & 5 & 5.5 \\
\hline \multirow{3}{*}{$Q[\mathrm{kN}]$} & "Deformable" & 36.7 & 70.1 & 76.8 & 111.3 & 167.5 & 22.5 & 45.8 & 107.0 \\
& "Design" & 33.8 & 64.3 & 70.3 & 101.4 & 151.3 & 21.3 & 43.4 & 100.2 \\
& "Rigid" & 33.1 & 62.8 & 68.6 & 98.8 & 147.3 & 21.0 & 42.7 & 98.4 \\
& $k \rightarrow \infty$ & 30.0 & 56.7 & 61.9 & 88.7 & 131.3 & 19.7 & 40.0 & 91.0 \\
\hline
\end{tabular}

Table 2. Summary of all the analytical results obtained for the geometries of [Genna and Gelfi 2012a] and [Bregoli 2014].

The reference numerical analyses, which replicate the experimental setup, have been run using the finite element code ABAQUS [Hibbitt et al. 2013], employing a full 3-dimensional mesh for all the parts. The core material was treated as elastic-plastic with nonlinear kinematic hardening; the analysis was run for arbitrarily large displacements and strains, under prescribed displacements. Coulomb friction was defined between the contacting surfaces, with friction coefficient $\mu=0.15$. More details about the numerical model can be found in [Bregoli 2014].

Simpler numerical analyses have also been run, again using ABAQUS, on planar beam models, in which the containment structure was described by rigid surfaces connected to each other by an elastic spring, and free to translate only. This was done in order to have a direct comparison between the results of the theoretical model and those of finite element simulations adopting exactly the same geometrical and material description. Therefore, in these analyses, that made use of linear Timoshenko beam elements, a linear kinematic hardening model was defined for the core material, and only two loading steps, from 0 to $-\Delta u$ and from $-\Delta u$ to $\Delta u$, with $\Delta u=11.2 \mathrm{~mm}$, were prescribed. The material parameters describe a metal that corresponds roughly to the real steel of the tests; the yield stress is $\sigma_{0}=330 \mathrm{MPa}$ and the hardening coefficient is $h=3850 \mathrm{MPa}$. These values have been adopted both for the analytical calculations and for the beam type numerical analyses; the value for the hardening coefficient $h$ was chosen corresponding to the maximum average strain applied to the BRB, i.e., $\bar{\varepsilon}=0.02$, even though the 
local strain could reach values twice as larger or more. An initial geometrical imperfection was defined as an irregularity of the axis line of the beam, of the order of $\pm s / 600$.

The calculation of the value for the stiffness constant $k$ of the elastic connection spring was done as illustrated in Appendix A, and adopting suitable values for the stiffness of the UPN160 profiles. For the "design" assembly, the value for the moment of inertia of a UPN160 profile, referred to its weakest principal axis, is $I_{e}=853000 \mathrm{~mm}^{4}$. For the case of the "deformable" assembly, in order to estimate the effects of the local deformability of the UPN webs, an equivalent moment of inertia was evaluated numerically, again using ABAQUS for a full 3D model of a single unreinforced UPN160 profile. The result thus obtained is $I_{e, \text { deform }}=466605 \mathrm{~mm}^{4}$. Finally, the equivalent moment of inertia of the containment profile, in the "rigid" configuration, was calculated to be $I_{e, \text { rigid }}=1120000 \mathrm{~mm}^{4}$.

The value of the bolt stiffness $k_{B}$ was also taken into account case by case, according to the various bolt geometries and properties.

Inserting all these parameters into (A.4) of Appendix A, one obtains an estimate of the elastic stiffness $k$ of the connection spring in the model of Figure 2. This value was adopted both in the analytical calculations and as a datum for the numerical analyses on the beam models.

Table 2 reports a summary of all the analytical results, as furnished by the procedure illustrated in Sections 4 and 5.

The table reports the calculated values of the stiffness $k$, of the axial force $F+\Delta F$ associated to $\Delta u=0.02 \mathrm{~mm}$ and accounting for the increase due to friction, of the semiwavelength $l_{0}$, of the wave number $N$, and of the total lateral thrust $Q$.

All the effects described in previous work [Genna and Gelfi 2012a; 2012b] can be recognized already for the limiting case of $k \rightarrow \infty$. Moreover, it is seen that, for decreasing values of $k$, the thrust increases progressively, the wave number remaining always constant.

The maximum difference in the predicted total thrust due to the difference in the external case stiffness occurs in the $5+1$ case, and is of about $28 \%$ between the "rigid" and the "deformable" setup. This suggests that, for this BRB, the "design" configuration should be appropriate.

Table 3 shows a comparison between the analytical results and the numerical ones given by the planar beam models. The largest differences appear in the cases $s=1 \mathrm{~mm}$ and for the smaller values of the stiffness $k$. All the geometries with $s=1 \mathrm{~mm}$ seem to offer a stronger challenge to the analytical model than the geometries with $s=0.25 \mathrm{~mm}$. In general, the larger is the gap $s$ the poorer is expected to be the approximation given by the analytical technique. This is due both to increasing lack of validity of a second-order theory and to possible lack of validity of Shanley's theory. Nevertheless, the analytical results of Table 3 appear always acceptable for sufficiently high values of $k$.

Table 4 reports a summary of numerical (3D), experimental, and analytical results. In the columns, the superscript (a) indicates that the experimental results have been taken from [Genna and Gelfi 2012a], whereas the superscript (b) indicates experimental results taken from [Bregoli 2014]. In both experimental and numerical results, when present, the superscripts (I) and (II) refer to the end of the first and the second compressive cycle of maximum amplitude, respectively. The numerical results refer only to the 3D analyses, that replicate the full AISC cyclic loading history [AISC 2010]. The analytical results marked as "best $k$ " are obtained adopting, for the stiffness $k$, the value corresponding to the actual containment case arrangement, as illustrated previously. 


\begin{tabular}{|l|rr|ll|ll|rr|}
\hline \multirow{2}{*}{ BRB Type } & \multicolumn{2}{|c|}{$F[\mathrm{kN}]$} & \multicolumn{2}{|c|}{$l_{0}[\mathrm{~mm}]$} & \multicolumn{2}{c|}{$N$} & \multicolumn{2}{c|}{$Q[\mathrm{kN}]$} \\
\cline { 2 - 9 } & $\begin{array}{c}\text { Beam } \\
\text { model }\end{array}$ & Anal. & $\begin{array}{c}\text { Beam } \\
\text { model }\end{array}$ & Anal. & $\begin{array}{c}\text { Beam } \\
\text { model }\end{array}$ & Anal. & $\begin{array}{r}\text { Beam } \\
\text { model }\end{array}$ & Anal. \\
\hline $5+0.25$ "Deformable" & 110.5 & 102.6 & 35 & 37.33 & 8 & 7.5 & 67.3 & 36.7 \\
$5+0.25$ "Design" & 106.4 & 102.4 & 35 & 37.33 & 8 & 7.5 & 43.0 & 33.8 \\
$5+0.25$ "Rigid" & 105.9 & 102.3 & 35 & 37.33 & 8 & 7.5 & 38.9 & 33.0 \\
$5+0.25 k \rightarrow \infty$ & 104.5 & 102.1 & 35 & 37.33 & 8 & 7.5 & 29.0 & 30.0 \\
$5+1$ "Deformable" & 112.1 & 110.5 & 40 & 37.33 & 7 & 7.5 & 101.6 & 167.5 \\
$5+1$ "Design" & 111.8 & 110.0 & 40 & 37.33 & 7 & 7.5 & 99.7 & 151.3 \\
$5+1$ "Rigid" & 111.7 & 109.5 & 40 & 37.33 & 7 & 7.5 & 97.4 & 147.3 \\
$5+1 k \rightarrow \infty$ & 112.3 & 108.7 & 40 & 37.33 & 7 & 7.5 & 92.5 & 131.3 \\
$7+0.25$ "Deformable" & 146.8 & 141.5 & 46.67 & 56 & 6 & 5 & 38.8 & 22.5 \\
$7+0.25$ "Design" & 145.5 & 141.5 & 46.67 & 56 & 6 & 5 & 29.1 & 21.3 \\
$7+0.25$ "Rigid" & 146.0 & 141.4 & 46.67 & 56 & 6 & 5 & 27.2 & 21.0 \\
$7+0.25 k \rightarrow \infty$ & 144.5 & 141.3 & 46.67 & 56 & 6 & 5 & 21.9 & 19.7 \\
$7+1$ "Deformable" & 147.9 & 147.2 & 46.67 & 50.91 & 6 & 5.5 & 86.7 & 107.0 \\
$7+1$ "Design" & 150.6 & 146.8 & 46.67 & 50.91 & 6 & 5.5 & 90.4 & 100.2 \\
$7+1$ "Rigid" & 151.1 & 146.7 & 46.67 & 50.91 & 6 & 5.5 & 89.5 & 98.4 \\
$7+1 k \rightarrow \infty$ & 151.4 & 146.3 & 46.67 & 50.91 & 6 & 5.5 & 82.0 & 91.0 \\
\hline
\end{tabular}

Table 3. Comparison between analytical predictions and numerical results. These last are given by planar beam models reproducing exactly the assembly of Figure 2, and adopting a linear kinematic hardening material model for the core.

The thrust values $Q$ obtained numerically agree well with the experimental results. In most cases, the analytical results with $k \rightarrow \infty$ provide acceptable estimates of the thrust, whereas the results obtained including a finite value of the stiffness $k$ can be somewhat off the mark, and more so as $k$ decreases and/or the gap $s$ increases, i.e., when the amplitude of the buckled shape increases.

Some analytical results (especially the $5+0.25$ "deformable" and $7+0.25$ cases) should be interpreted with caution, because for those there is some uncertainty about the actual value of the nominal gap $s$, which was not controlled after the BRB assemblage. Nevertheless, considering also that the experimental values of the lateral thrust can change significantly just after a repetition of the same cycle, for the same value of $\Delta u$ - a phenomenon that has not been described, in the calculations developed here - the overall accuracy of the analytical results appears acceptable from the engineering viewpoint.

As said, there is another effect that influences in a significant way the value of the lateral thrust in BRBs subjected to cyclic loading. As shown also in Table 4, and confirmed by other published and unpublished results, the lateral thrust value depends not only on the current value of the axial shortening $\Delta u$, but also on the number of loading cycles applied to the BRB at constant value of $\Delta u$. At each repetition of a cycle with the same amplitude, the thrust $Q$ increases, probably as an effect of the accumulation of plastic dissipation, which leads to a progressive change of geometry of the core. One of the consequences of this effect is shown in the last rows of Table 4, which report the increase $\Delta s$ of the nominal gap, at 


\begin{tabular}{|c|c|c|c|c|c|c|c|c|c|c|c|c|}
\hline & & \multicolumn{3}{|c|}{$5+0.25$} & \multirow{2}{*}{\begin{tabular}{|l|}
$5+0.46$ \\
Des. $^{(b)}$
\end{tabular}} & \multicolumn{2}{|c|}{$5+0.5$} & \multirow{2}{*}{$\begin{array}{l}5+0.7 \\
\text { Des. }^{(b)}\end{array}$} & \multirow{2}{*}{$\frac{5+1}{\text { Des. }^{(a)}}$} & \multirow{2}{*}{$\begin{array}{l}7+0.25 \\
\text { Des. }^{(a)}\end{array}$} & \multirow{2}{*}{$\frac{7+0.5}{\text { Des. }^{(a)}}$} & \multirow{2}{*}{$\frac{7+1}{\text { Des. }^{(a)}}$} \\
\hline & & Def. $^{\text {(b) }}$ & Des. ${ }^{(b)}$ & Rigid $^{(b)}$ & & Des. ${ }^{\text {(a) }}$ & Rigid $^{(b)}$ & & & & & \\
\hline \multirow{4}{*}{$\frac{Z}{Z}$} & Exper. & $\begin{array}{l}106.4^{(\mathrm{I})} \\
108.6^{(\mathrm{II})}\end{array}$ & $110.5^{(\mathrm{II})}$ & $\begin{array}{l}107.2^{(\mathrm{I})} \\
108.8^{(\mathrm{II})}\end{array}$ & \begin{tabular}{|l|}
$106.5^{(\mathrm{I})}$ \\
$110.1^{(\mathrm{II})}$
\end{tabular} & 121.5 & $\begin{array}{l}110.1^{(\mathrm{I})} \\
112.9^{(\mathrm{II})}\end{array}$ & \begin{tabular}{|l|}
$106.3^{(\mathrm{I})}$ \\
$107.4^{(\mathrm{II})}$ \\
\end{tabular} & 123.0 & 158.8 & 157.4 & 160.8 \\
\hline & Num. & & & $\begin{array}{l}114.6^{(\mathrm{I})} \\
114.8^{(\mathrm{II})}\end{array}$ & \begin{tabular}{|l|}
$116.4^{(\mathrm{I})}$ \\
$115.7^{(\mathrm{II})}$
\end{tabular} & & $\begin{array}{l}121.7^{(\mathrm{I})} \\
121.7^{(\mathrm{II})}\end{array}$ & \begin{tabular}{|l|}
$122.7^{(\mathrm{I})}$ \\
$106.6^{(\mathrm{II})}$ \\
\end{tabular} & & & & \\
\hline & $\begin{array}{l}\text { Analyt., } \\
\text { best } k\end{array}$ & 102.6 & 102.4 & 102.3 & 104.4 & 104.8 & 104.7 & 106.8 & 109.7 & 141.5 & 143.0 & 146.8 \\
\hline & $\begin{array}{l}\text { Analyt., } \\
k \rightarrow \infty\end{array}$ & & 102.1 & & 103.9 & 10 & 4.3 & 106.0 & 108.7 & 141.3 & 142.8 & 146.3 \\
\hline \multirow{4}{*}{$\begin{array}{l}\bar{\Xi} \\
\text { 窇 }\end{array}$} & Exper. & \begin{tabular}{|l|}
$37.5^{(\mathrm{I})}$ \\
$37.33^{(\mathrm{II})}$
\end{tabular} & $35^{(\mathrm{II})}$ & $35^{(\mathrm{II})}$ & $\begin{array}{l}33^{(\mathrm{I})} \\
33^{(\mathrm{II})}\end{array}$ & 37.3 & $40^{(\mathrm{II})}$ & $\begin{array}{l}35^{\text {(I) }} \\
35^{\text {(II) }}\end{array}$ & 46.65 & 50.91 & 50.91 & 50.91 \\
\hline & Num. & & & $\begin{array}{l}40^{(\mathrm{I})} \\
40^{\text {(II) }}\end{array}$ & $\begin{array}{l}40^{(\mathrm{I})} \\
40^{(\mathrm{II})}\end{array}$ & & $\begin{array}{l}40^{(\mathrm{I})} \\
40^{(\mathrm{II})}\end{array}$ & $\begin{array}{l}40^{(\mathrm{I})} \\
40^{\text {(II) }}\end{array}$ & & & & \\
\hline & $\begin{array}{l}\text { Analyt., } \\
\text { best } k\end{array}$ & 37.33 & 37.33 & 37.33 & 37.33 & 37.33 & 37.33 & 37.33 & 37.33 & 56 & 56 & 50.91 \\
\hline & $\begin{array}{l}\text { Analyt., } \\
k \rightarrow \infty\end{array}$ & & 37.33 & & 37.33 & 37. & 33 & 37.33 & 37.33 & 56 & 56 & 50.91 \\
\hline \multirow{4}{*}{$z$} & Exper. & $7.5^{(\mathrm{II})}$ & $8^{(\mathrm{II})}$ & $8^{(\mathrm{II})}$ & $\begin{array}{l}8.5^{(\mathrm{I})} \\
8.5^{(\mathrm{II})}\end{array}$ & 7.5 & $7^{(\mathrm{II})}$ & $\begin{array}{l}8^{(\mathrm{I})} \\
8^{(\mathrm{II})} \\
\end{array}$ & 6 & 5.5 & 5.5 & 5.5 \\
\hline & Num. & & & $\begin{array}{l}7.5^{(\mathrm{I})} \\
7.5^{(\mathrm{II})}\end{array}$ & $\begin{array}{l}7.5^{(\mathrm{I})} \\
7.5^{(\mathrm{II})}\end{array}$ & & $\begin{array}{l}7.5^{(\mathrm{I})} \\
7.5^{(\mathrm{II})}\end{array}$ & $\begin{array}{l}7.5^{(\mathrm{I})} \\
7.5^{(\mathrm{II})}\end{array}$ & & & & \\
\hline & $\begin{array}{l}\text { Analyt., } \\
\text { best } k\end{array}$ & 7.5 & 7.5 & 7.5 & 7.5 & 7.5 & 7.5 & 7.5 & 7.5 & 5 & 5 & 5.5 \\
\hline & $\begin{array}{l}\text { Analyt., } \\
k \rightarrow \infty\end{array}$ & & 7.5 & & 7.5 & 7. & 5 & 7.5 & 7.5 & 5 & 5 & 5.5 \\
\hline \multirow{4}{*}{$\frac{\bar{Z}}{a}$} & Exper. & $\begin{array}{l}52.1^{(\mathrm{I})} \\
61.9^{(\mathrm{II})}\end{array}$ & $\begin{array}{l}31.4^{(\mathrm{I})} \\
37.5^{(\mathrm{II})}\end{array}$ & $\begin{array}{l}35.5^{(\mathrm{I})} \\
41.3^{(\mathrm{II})}\end{array}$ & $\begin{array}{l}49.8^{(\mathrm{I})} \\
66.2^{(\mathrm{II})}\end{array}$ & 93.7 & $\begin{array}{l}67.7^{(\mathrm{I})} \\
77.1^{\text {(II) }}\end{array}$ & $\begin{array}{l}83.3^{(\mathrm{I})} \\
86.6^{(\mathrm{II})}\end{array}$ & 136.4 & 63.6 & 61.0 & 121.1 \\
\hline & Num. & & & $\begin{array}{l}32.4^{(\mathrm{I})} \\
34.2^{(\mathrm{II})} \\
\end{array}$ & $\begin{array}{l}58.1^{(\mathrm{I})} \\
61.7^{(\mathrm{II})} \\
\end{array}$ & & $\begin{array}{l}64.7^{(\mathrm{I})} \\
71.1^{(\mathrm{II})} \\
\end{array}$ & \begin{tabular}{|l|}
$89.6^{(\mathrm{I})}$ \\
$96.7^{(\mathrm{II})}$ \\
\end{tabular} & & & & \\
\hline & $\begin{array}{l}\text { Analyt., } \\
\text { best } k\end{array}$ & 36.7 & 33.8 & 33.1 & 64.3 & 70.3 & 68.6 & 101.4 & 151.3 & 21.3 & 43.4 & 100.2 \\
\hline & $\begin{array}{l}\text { Analyt., } \\
k \rightarrow \infty\end{array}$ & & 30.0 & & 56.7 & 61 & .9 & 88.7 & 131.3 & 19.7 & 40.0 & 91.0 \\
\hline \multirow{3}{*}{$\frac{\Xi}{\Xi}$} & Exper. & 1.0 & 0.09 & 0.07 & 0.16 & & 0.18 & 0.23 & & & & \\
\hline & Num. & & & 0.063 & 0.14 & & 0.15 & 0.21 & & & & \\
\hline & $\begin{array}{l}\text { Analyt., } \\
\text { best } k\end{array}$ & $\begin{array}{c}0.054 \\
(0.216 s)\end{array}$ & $\begin{array}{l}0.031 \\
(0.124 s)\end{array}$ & $\begin{array}{r}0.025 \\
(0.1 s)\end{array}$ & $\begin{array}{c}0.058 \\
(0.126 s)\end{array}$ & $\begin{array}{c}0.064 \\
(0.128 s)\end{array}$ & $\begin{array}{c}0.051 \\
(0.102 s)\end{array}$ & $\begin{array}{c}0.092 \\
(0.131 s)\end{array}$ & $\begin{array}{c}0.137 \\
(0.137 s)\end{array}$ & $\begin{array}{l}0.02 \\
(0.086 s)\end{array}$ & $\begin{array}{l}0.041 \\
(0.082 s)\end{array}$ & $\begin{array}{l}0.095 \\
(0.095 s)\end{array}$ \\
\hline
\end{tabular}

Table 4. Comparison of numerical, experimental, and analytical results. Numerical results are given by 3D FE models that replicate the experimental setup. Experimental results marked (a) are from [Genna and Gelfi 2012a]; those marked ${ }^{\text {(b) }}$ are from [Bregoli 2014]. Superscript ${ }^{\text {I }}$ and ${ }^{\text {II }}$ refer to the first and second cycles of maximum amplitude. The rows show, from top to bottom: maximum axial compressive force $F$; semiwavelength $l_{0}$; number $N$ of buckled waves; total lateral thrust $Q$; increase $\Delta s$ in the nominal gap $s$ due to deflection of the containment profiles. 
each side of the buckled core, due to the deformation of the external profiles. Both the experimental and numerical results are systematically much higher than the analytical ones. This is certainly due to some inaccuracy in the estimate of the elastic stiffness of the containment structure, as well as to the approximations inherent into the analytical procedure; but it is certainly also due to the fact that the analytical procedure neglects the increase of the lateral thrust produced by the accumulation of plastic strains at each repetition of the same loading cycle. This aspect deserves more attention, and is currently under investigation.

It is worth remarking that even though the analytical predictions for $\Delta s$ are systematically too small, they indicate a displacement $\Delta s$ of the order of 10 to $20 \%$ of the nominal gap, showing that the deformability of the external profiles plays an important role, that can not be neglected when computing the lateral thrust.

Appendix B will report an example of application of the proposed procedure to a full-scale BRB, in order to illustrate the calculations to be done by an engineer when designing a "real" BRB.

\section{Conclusions}

The theoretical results developed in [Genna and Bregoli 2014] have been adopted, and suitably extended, to obtain engineering usable estimates for the lateral thrust in steel BRBs. The new expressions derived here for such thrust overcome several important limitations of previous work:

- The elastic stiffness of the containment structure is explicitly accounted for.

- The presence of line contacts is explicitly considered in the expressions for the buckled wavelength.

- An important modification, in the calculation of the buckled wavelength and of the associated value of the lateral thrust, is introduced here, in order to take into account the expected presence of tensilecompressive loading cycles, accompanied by the development of plastic strains of both signs.

All these developments allow for analytical estimates that appear consistently acceptable from the engineering viewpoint, with the important limitation of being valid only in situations implying small buckled amplitudes. Therefore, both cases of a too large nominal gap and/or of an insufficiently stiff containment assembly might prevent the applicability of the analytical technique. A condition was defined to individuate a lower limit of the containment stiffness to be employed, which should be coupled with other possible conditions, such as, for example, the one that guarantees the absence of global buckling or of plastic collapse of the BRB under the prescribed axial shortening.

The analysis of the mechanical behavior of BRBs is a relatively new and challenging research topic, with many aspects to be considered. The present work constitutes an attempt at development and synthesis of previous theoretical work, and should provide a good engineering approach to the analytical calculation of the lateral thrust in steel BRBs. More work is still in progress, concerned with several other topics of interest:

- the accumulation of experimental evidence about several details still not fully explored, such as the dependency of the lateral thrust on the loading history and on several other factors;

- the understanding of the real loading condition to be applied in the analysis of BRBs;

- the development of reasonably accurate yet not too expensive numerical models for this type of problems; 
- the acquisition of a deeper understanding of all the theoretical aspects involved in this type of problems; an example of these is suggested, for instance, in [Chai 1998], where it is pointed out that even in the case of a fully conservative situation (linear elasticity for all the materials and frictionless unilateral contact) the postbuckled behavior of BRBs, under loading-unloading cycles, might entail some form of hysteresis loop, of unclear explanation;

- the understanding, both experimentally and numerically, of the low-cycle fatigue behavior of the buckled core, and specially the possibility of predicting the number of cycles before its failure.

All these aspects, for which there is no space in the present work, deserve more study, which is currently under way.

\section{Appendix A: Example of calculation of the spring stiffness $k$}

An example of how to compute a stiffness value $k$ accounting for the elastic stiffness of the containment structure exploits the scheme of Figure 8, which considers two retention profiles with two bolted connections. Other configurations, possibly with more bolts along the containment profiles axis, could be found in practical applications; in these cases it is necessary to suitably modify the expressions proposed next, and, in particular, result (A.1).

One possible way to relate the stiffness $k$ to the stiffness $k_{B}$ of the connection bolts at each side, and to the elastic bending stiffness $E_{e} I_{e}$ of the external profiles, is to make the total displacement $\eta_{m}$, at each side of the model of Figure 2, due to the total thrust $Q$, equal to the total displacement $\eta_{e}$, at each side of the

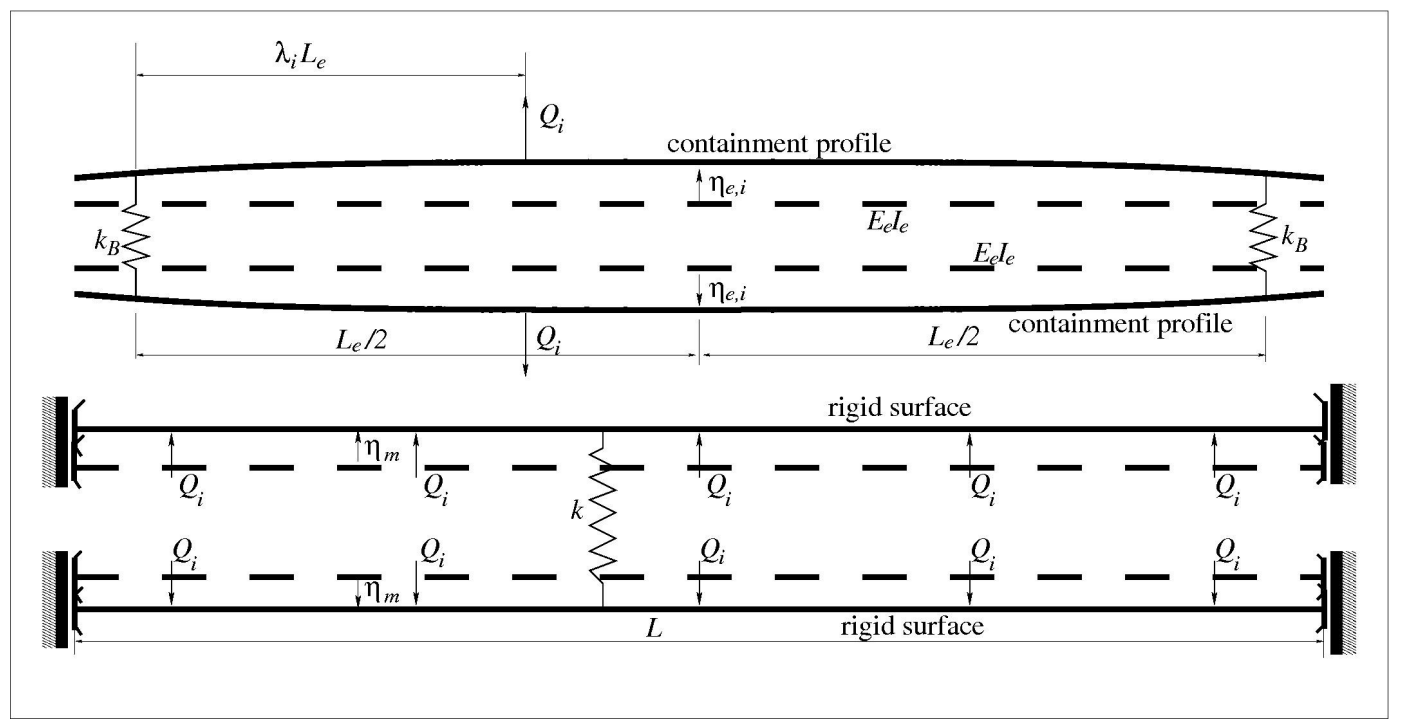

Figure 8. Top image: structural scheme of the external containment structure in the case of two profiles connected by two bolts in the central portion, loaded by a unit thrust $Q_{i}$ located at an arbitrary position along the BRB length. Bottom image: model of Figure 2, rigid surfaces only, loaded by the total thrust $Q$. Each rigid surface translates by $\eta_{m}=Q /(2 k)$. 
real structural arrangement, under the same thrust, evaluated at the mid-point along the external profile axis. The symbol $L_{e}$ denotes the distance between the connection bolts in the real structural arrangement. Contact can occur both in the external and in the central portions of the containment profiles, i.e., both outside and between the two bolts.

One can then exploit the assumption of periodicity of the buckled shape of the core, which implies that all the unit thrust forces $Q_{i}$ have the same value at all the contact points. The displacement $\eta_{e, i}$ at the mid-point of each lateral containment profile, due to a single force $Q_{i}$ placed at a distance $\lambda_{i} L$ from its left extremity, with $1 \leq i \leq N / 2$, as in Figure 8, has the following expression:

$$
\begin{gathered}
\eta_{e, i}=Q_{i}\left(\frac{1}{4 k_{B}}+\frac{c_{i, e}}{32 E_{e} I_{e}}+\frac{c_{i, i}}{96 E_{e} I_{e}}\right), \\
c_{i, e}=2 \lambda_{i} L L_{e}^{2}+L_{e}^{3}-L_{e}^{2} L, \quad 0 \leq \lambda_{i} \leq \frac{L-L_{e}}{2 L}, \\
c_{i, i}=2 L_{e}^{3}-3 L_{e} L^{2}+L^{3}+12 \lambda_{i}^{2} L^{2}\left(L-L_{e}\right)-6 \lambda_{i} L^{2}\left(L-2 L_{e}\right)-8 \lambda_{i}^{3} L^{3}, \\
\frac{L-L_{e}}{2 L} \leq \lambda_{i} \leq \frac{1}{2} .
\end{gathered}
$$

Then, the total displacement $\eta_{e}$, due to a number $N$ of unit forces $Q_{i}$ of equal intensity, has the following expression:

$$
\eta_{e}=Q\left(\frac{1}{4 k_{B}}+\frac{1}{48 E_{e} I_{e} N} \sum_{i=1}^{N^{*}} c_{i, e}+\frac{1}{96 E_{e} I_{e} N} \sum_{i=N^{*}+1}^{N} c_{i, i}\right),
$$

where $N^{*}$ denotes the number of contact points outside the bolts. Since the corresponding opening in the model of Figure 2 is given by

$$
\eta_{m}=\frac{Q}{2 k}
$$

by equating the results of (A.2) and (A.3) it is immediate to obtain

$$
k=\frac{48 E_{e} I_{e} N k_{B}}{24 E_{e} I_{e} N+3 k_{B} \sum_{i=1}^{N^{*}} c_{i, e}+k_{B} \sum_{i=N^{*}+1}^{N} c_{i, i}} .
$$

The values $\lambda_{i}$ that appear in coefficients $c_{i, e}$ and $c_{i, i}$ in expressions (A.1) to (A.4) can be easily related to the total number of buckled waves, $N$, exploiting once more the assumption of periodicity of the buckled shape. One thus finds

$$
\lambda_{i}=\frac{1}{2 N}+\frac{i-1}{N}, \quad 0 \leq \lambda_{i} \leq \frac{1}{2}, \quad 1 \leq i \leq \frac{N}{2} .
$$

The values of $\lambda_{i}$ corresponding to the contact forces located in the right portion of the structure are then obtained from symmetry.

The value for $k_{B}$ depends on the bolt configuration employed in the real BRB, and is of course related to the elementary expression holding for a single bolt of area $A_{B}$, Young modulus $E_{B}$ and length $l_{B}$, i.e.,

$$
k_{B}=\frac{E_{B} A_{B}}{l_{B}} .
$$



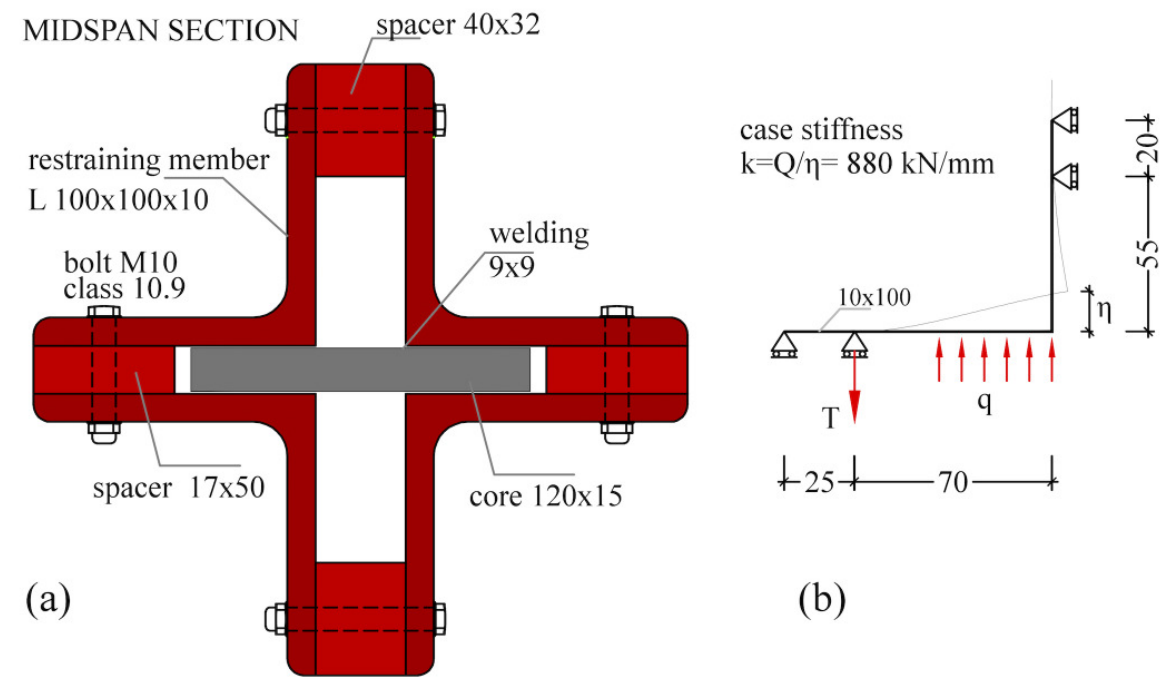

(b)

Figure 9. Geometrical details of the section of a full-scale BRB (a); and scheme for the calculation of the case local stiffness (b).

Since the number $N$ of buckled waves is unknown, at the moment of estimating the stiffness $k$ as in (A.4), one must iterate, at least in principle, in order to reach a working expression. One can start from an estimate with $N=1$, or, better, compute $N$ assuming $k \rightarrow \infty$, and then iterate if necessary to reach a final value for $N$ and then of the total thrust $Q$.

This elementary calculation has neglected a possible contribution to the elastic stiffness $k$ deriving from the local deformability of the containment at each contact point. This could be due to three-dimensional effects (i.e., plate behavior) possibly important, depending on the actual design of the containment profiles; if necessary, this contribution should be computed case by case adopting specific techniques, either analytical or numerical, for instance based on plate bending theory.

\section{Appendix B: Design of the connecting bolts in a full scale all-steel BRB}

This appendix illustrates the design of the bolts connecting the restraining profiles of a real all-steel BRB, following the procedure presented in the paper for the calculation of the lateral thrust exerted by the core during its high-mode buckling. Two cases are presented: in the former, symmetric axial displacement cycles of the BRB core are considered, as occurring in a quasistatic cyclic test following the AISC loading protocol; in the latter, the lateral thrust is calculated for a BRB core undergoing nonsymmetric axial displacement cycles, such as it may happen in reality under a seismic event [Lin et al. 2012].

Figure 9 shows the details of the considered BRB, which was designed and tested at University of Brescia [Gelfi and Metelli 2007]. The brace is $6.0 \mathrm{~m}$ long, with a dissipative core length $L=3.0 \mathrm{~m}$. The considered gap is $s=1.0 \mathrm{~mm}$. The four L-shaped members that act as a retaining structure are connected by means of high strength 10.9 bolts having a diameter of $10 \mathrm{~mm}$, whose spacing must be calculated on the basis of the lateral thrust generated by the core on the restraining profiles. The core is made of 
S275 grade steel, with a nominal yield strength $f_{y m}=321 \mathrm{MPa}$. The friction coefficient between the lubricated surfaces is taken equal to $\mu=0.15$.

This brace is designed to be employed in the first floor of a seven-story building located in a typical medium-seismic zone in Northern Italy (soil type $C$, peak ground acceleration $a_{g}=0.20 \mathrm{~g}$ ). Details of the design of this BRB are to be found in [Gelfi et al. 2007; Gelfi and Metelli 2007]. The adopted Young modulus of the material is $E=210000 \mathrm{MPa}$; for $\bar{\varepsilon}=0.02$ [AISC 2010], the hardening coefficient was taken equal to $h=3850 \mathrm{MPa}$, and the core yield strength is $f_{y}=R_{y} f_{y k}=1.15 \times 275=316 \mathrm{MPa}$, $R_{y}=1.15$ being the material overstrength factor [AISC 2010]. The restraining member will first be considered as infinitely rigid $(k \rightarrow \infty)$, then as having a finite stiffness, in order to highlight the differences entailed by this factor. The calculation of a finite value for the stiffness $k$ is based on the scheme of Figure 9, and was done numerically. The resulting value is $k=880 \mathrm{kN} / \mathrm{mm}$.

Example 1: symmetric cycles [AISC 2010]. By considering the BRB core undergoing an average axial core strain $\bar{\varepsilon}=0.02$ with cycles of equal amplitude in tension and compression, as required by the AISC loading protocol, the presented design procedure gives the following results.

- Infinitely rigid containment profiles $(k \rightarrow \infty)$ :

(1) $F=729 \mathrm{kN}$ is the maximum axial compressive force accounting also for the increase due to friction;

(2) $l_{0}=115.4 \mathrm{~mm}$ is the predicted semiwavelength of the buckled shape under the maximum axial shortening;

(3) $N=13$ is the predicted number of contact points at each side of the core;

(4) $q=0.165 \mathrm{kN} / \mathrm{mm}$ is the predicted lateral thrust per unit length.

- Deformable containment profiles $(k=880 \mathrm{kN} / \mathrm{mm})$ :

(1) $F=741.5 \mathrm{kN}$ is the maximum axial compressive force accounting also for the increase due to friction;

(2) $l_{0}=115.4 \mathrm{~mm}$ is the predicted semiwavelength of the buckled shape under the maximum axial shortening;

(3) $N=13$ is the predicted number of contact points at each side of the core;

(4) $q=0.237 \mathrm{kN} / \mathrm{mm}$ is the predicted lateral thrust per unit length;

(5) $\Delta s=0.4 \mathrm{~mm}$ is the increase of the gap due to the opening between the containment profiles. Such an increase is the main responsible for the significant increase (order of $40 \%$ ) of the lateral thrust with respect to the value given by $k \rightarrow \infty$; this shows how, in this case, the deformability of the retaining case plays an important role.

The total tensile force $T$ acting in the bolts is also calculated on the basis of the scheme of Figure 9, including contributions due to the prying action, according to [CEN 2005]. This calculation yields $T=29.63 \mathrm{kN}$. By using high strength bolts $\left(f_{u b}=1000 \mathrm{MPa}\right)$, two M10 bolts with a longitudinal spacing $p=100 \mathrm{~mm}$ are needed, having calculated their tensile strength as $F_{t, R d}=0.9 f_{u b} A_{s} / \gamma_{M 2}=41.76 \mathrm{kN}$.

It is interesting to compare these results with the corresponding ones obtained experimentally for this type of BRB, that will be described elsewhere. These are: maximum axial compressive force $F=825 \mathrm{kN}$; average value of the buckled semiwavelength $l_{0}=113 \mathrm{~mm}$; estimated value of the total lateral thrust (not measured experimentally), from (23): $Q=2 \Delta F / \mu=1080 \mathrm{kN}$, where $\Delta F$ is the experimentally 
measured difference between the peak axial forces in compression and in tension, to be compared with the analytical prediction $Q=q L=711 \mathrm{kN}$ for the case of $k=880 \mathrm{kN} / \mathrm{mm}$.

Example 2: nonsymmetric displacement cycles. In order to understand the influence of the cycle type on the predicted response of a BRB, we consider now the case of a nonsymmetric axial displacement cycle, such as it is expected to occur under a real seismic event. Lin et al. [2012] report results of tests performed on BRBs subjected to a seismic excitation; from these results it can be seen that, ignoring a possible global shift of the displacement maximum values, the ratio between the maximum tensile average strain and the maximum compressive one, in a typical cycle, is of about 0.375 . Therefore, we keep fixed the value $\bar{\varepsilon}=0.02$ as the maximum compressive axial strain, which governs the current value of the axial force, but we consider a strain cycle ranging between $-\bar{\varepsilon}_{t}=-0.007$ and $\bar{\varepsilon}_{c}=0.02$. Therefore, use is made of (20) instead of (21) to calculate the driving stress for the calculation of the wavelength. The corresponding results are as follows.

- Infinitely rigid containment profiles $(k \rightarrow \infty)$ :

(1) $F=727 \mathrm{kN}$ is the maximum axial compressive force accounting also for the increase due to friction;

(2) $l_{0}=120 \mathrm{~mm}$ is the predicted semiwavelength of the buckled shape under the maximum axial shortening;

(3) $N=12.5$ is the predicted number of contact points at each side of the core;

(4) $q=0.149 \mathrm{kN} / \mathrm{mm}$ is the predicted lateral thrust per unit length.

- Deformable containment profiles $(k=880 \mathrm{kN} / \mathrm{mm})$ :

(1) $F=735 \mathrm{kN}$ is the maximum axial compressive force accounting also for the increase due to friction;

(2) $l_{0}=120 \mathrm{~mm}$ is the predicted semiwavelength of the buckled shape under the maximum axial shortening;

(3) $N=12.5$ is the predicted number of contact points at each side of the core;

(4) $q=0.205 \mathrm{kN} / \mathrm{mm}$ is the predicted lateral thrust per unit length;

(5) $\Delta s=0.35 \mathrm{~mm}$ is the increase of the gap due to the opening between the containment profiles.

The compressive axial force remains, as expected, practically unaltered, with respect to the case of symmetric cycles ending to the same shortening displacement; the lateral thrust reduces of about $15 \%$, as a consequence of the reduced strain excursion in tension. Since the design of this element must comply with the AISC regulations, which prescribe tests under symmetrical loading cycles, this last set of calculations should be seen only as a check, and not as a design procedure.

\section{Acknowledgments}

This work was done with the support of the Italian Ministry of Education and Research (MIUR). The authors express their thanks to Dr. Marco Baiguera for his help with both the experimental and the numerical part of the work.

\section{References}

[AISC 2010] Seismic provisions for structural steel buildings, American Institute of Steel Construction, Chicago, USA, 2010. 
[Bregoli 2014] G. Bregoli, Studio di dispositivi in acciaio per il miglioramento sismico di edifici in C.A., Ph.D. thesis, University of Brescia, Italy, 2014.

[Bregoli et al. 2014] G. Bregoli, M. Baiguera, G. Metelli, and F. Genna, "Experimental and numerical analysis on the core lateral thrust in bolted BRBs", in Proceedings of the 7th European Conference on Steel and Composite Structures (EUROSTEEL 2014) (Napoli, Italy, 2014), University of Naples Federico II, 2014.

[CEN 2005] "EN1993-1-8 - Eurocode 3: design of steel structures, 1-8: design of joints", technical report, European Committee for Standardization, Brussels, 2005, Available at http://www.eurocodes.fi/1993/1993-1-8/SFS-EN1993-1-8-AC.pdf.

[Chai 1998] H. Chai, "The post-buckling response of a bi-laterally constrained column”, J. Mech. Phys. Solids 46:7 (1998), $1155-1181$.

[Chou and Chen 2010] C.-C. Chou and S.-Y. Chen, "Subassemblage tests and finite element analyses of sandwiched bucklingrestrained braces", Eng. Struct. 32:8 (2010), 2108 - 2121.

[Gelfi and Metelli 2007] P. Gelfi and G. Metelli, "Prova sperimentale di un elemento diagonale di controvento ad instabilità controllata", pp. 169-176 in Atti del XXI Congresso C.T.A.: Costruire con l'Acciaio (Catania, Italy, 2007), edited by A. Ghersi, Logo Flaccovio, Milan, 2007.

[Gelfi et al. 2007] P. Gelfi, E. Marchina, G. Metelli, and A. Temponi, "Studio teorico e sperimentale sui controventi concentrici ad instabilità impedita", technical report, no. 5, DICATAM, University of Brescia, Brescia, Italy, 2007.

[Genna and Bregoli 2014] F. Genna and G. Bregoli, "Small amplitude elastic buckling of a beam under monotonic axial loading, with frictionless contact against movable rigid surfaces", JOMMS 9:4 (2014), 441-463.

[Genna and Gelfi 2012a] F. Genna and P. Gelfi, "Analysis of the lateral thrust in bolted steel buckling-restrained braces, I: experimental and numerical results", J. Struct. Eng. (ASCE) 138:10 (2012), 1231-1243.

[Genna and Gelfi 2012b] F. Genna and P. Gelfi, "Analysis of the lateral thrust in bolted steel buckling-restrained braces, II: engineering analytical estimates”, J. Struct. Eng. (ASCE) 138:10 (2012), 1244-1254.

[Hibbitt et al. 2013] H. Hibbitt, B. Karlsson, and P. Sorensen, “ABAQUs User's Manual”, Software manual (release 6.12), Dassault Systèmes, Simulia Corp, Providence, RI, 2013.

[Lin et al. 2012] P.-C. Lin, K.-C. Tsai, K.-J. Wang, Y.-J. Yu, C.-Y. Wei, A.-C. Wu, C.-Y. Tsai, C.-H. Lin, J.-C. Chen, A. H. Schellenberg, S. A. Mahin, and C. W. Roeder, "Seismic design and hybrid tests of a full-scale three-story buckling-restrained braced frame using welded end connections and thin profile", Earthq. Eng. Struct. Dyn. 41:5 (2012), 1001-1020.

[Shanley 1947] F. Shanley, "Inelastic column theory", J. Aeronaut. Sci. 14 (1947), 261-268.

[Takeuchi et al. 2010] T. Takeuchi, J. Hajjar, R. Matsui, K. Nishimoto, and I. Aiken, "Local buckling restraint condition for core plates in buckling restrained braces", J. Constr. Steel Res. 66:2 (2010), 139-149.

[Wu et al. 2014] A.-C. Wu, P.-C. Lin, and K.-C. Tsai, "High-mode buckling responses of buckling-restrained brace core plates", Earthq. Eng. Struct. Dyn. 43:3 (2014), 375-393.

[Zhao et al. 2014] J. Zhao, B. Wu, W. Li, and J. Ou, "Local buckling behavior of steel angle core members in bucklingrestrained braces: cyclic tests, theoretical analysis, and design recommendations", Eng. Struct. 66 (2014), 129-145.

Received 6 Oct 2015. Revised 17 Nov 2015. Accepted 24 Nov 2015.

GUIDO BREGOLI: g.bregoli@unibs.it

Department of Civil Engineering, University of Brescia, Via Branze, 43, I-25123 Brescia, Italy

FRANCESCO GENNA: francesco.genna@unibs.it

Department of Civil Engineering, University of Brescia, Via Branze 43, I-25123 Brescia, Italy

Giovanni Metelli: giovanni.metelli@unibs.it

Department of Civil Engineering, University of Brescia, Via Branze, 43, I-25123 Brescia, Italy 


\section{SUBMISSION GUIDELINES}

\section{ORIGINALITY}

Authors may submit manuscripts in PDF format online at the Submissions page. Submission of a manuscript acknowledges that the manuscript is original and has neither previously, nor simultaneously, in whole or in part, been submitted elsewhere. Information regarding the preparation of manuscripts is provided below. Correspondence by email is requested for convenience and speed. For further information, write to contact@msp.org.

\section{LANGUAGE}

Manuscripts must be in English. A brief abstract of about 150 words or less must be included. The abstract should be self-contained and not make any reference to the bibliography. Also required are keywords and subject classification for the article, and, for each author, postal address, affiliation (if appropriate), and email address if available. A home-page URL is optional.

\section{FORMAT}

Authors can use their preferred manuscript-preparation software, including for example Microsoft Word or any variant of $\mathrm{T}_{\mathrm{E}} \mathrm{X}$. The journal itself is produced in $\mathrm{LT}_{\mathrm{E} X} \mathrm{X}$, so accepted articles prepared using other software will be converted to $\mathrm{LT}_{\mathrm{E}} \mathrm{X}$ at production time. Authors wishing to prepare their document in $\mathrm{ET}_{\mathrm{E}} \mathrm{X}$ can follow the example file at www.jomms.net (but the use of other class files is acceptable). At submission time only a PDF file is required. After acceptance, authors must submit all source material (see especially Figures below).

\section{REFERENCES}

Bibliographical references should be complete, including article titles and page ranges. All references in the bibliography should be cited in the text. The use of BibTEX is preferred but not required. Tags will be converted to the house format (see a current issue for examples); however, for submission you may use the format of your choice. Links will be provided to all literature with known web locations; authors can supply their own links in addition to those provided by the editorial process.

\section{FIGURES}

Figures must be of publication quality. After acceptance, you will need to submit the original source files in vector format for all diagrams and graphs in your manuscript: vector EPS or vector PDF files are the most useful. (EPS stands for Encapsulated PostScript.)

Most drawing and graphing packages-Mathematica, Adobe Illustrator, Corel Draw, MATLAB, etc.-allow the user to save files in one of these formats. Make sure that what you're saving is vector graphics and not a bitmap. If you need help, please write to graphics@msp.org with as many details as you can about how your graphics were generated.

Please also include the original data for any plots. This is particularly important if you are unable to save Excelgenerated plots in vector format. Saving them as bitmaps is not useful; please send the Excel (.xls) spreadsheets instead. Bundle your figure files into a single archive (using zip, tar, rar or other format of your choice) and upload on the link you been given at acceptance time.

Each figure should be captioned and numbered so that it can float. Small figures occupying no more than three lines of vertical space can be kept in the text ("the curve looks like this:"). It is acceptable to submit a manuscript with all figures at the end, if their placement is specified in the text by means of comments such as "Place Figure 1 here". The same considerations apply to tables.

\section{WHITE SPACE}

Forced line breaks or page breaks should not be inserted in the document. There is no point in your trying to optimize line and page breaks in the original manuscript. The manuscript will be reformatted to use the journal's preferred fonts and layout.

\section{ProOfs}

Page proofs will be made available to authors (or to the designated corresponding author) at a Web site in PDF format. Failure to acknowledge the receipt of proofs or to return corrections within the requested deadline may cause publication to be postponed. 


\title{
Journal of Mechanics of Materials and Structures
}

\author{
Volume 11, No. $2 \quad$ March 2016
}

The effect of small scale on the free vibration of functionally graded truncated conical shells

Yaghoub TAdi Beni and Fahimeh Mehralian

Conditions for the localisation of plastic deformation in temperature sensitive viscoplastic materials MARTIN K. PAESOLd, ANDREW P. BASSOM, KLAUS RegenaUeR-Lieb and Manolis VeVEAKIS

A simple hard-particle collision model with a smooth transition to full slip

Multiobjective optimization of laminated composite plate with elliptical cut-out using ANN based NSGA-II

P. Emmanuel Nicholas, M. C. Lenin Babu and A. Sathya Sofia

Analytical estimates for the lateral thrust in bolted steel buckling-restrained braces Guido Bregoli, Francesco Genna and Giovanni Metelli 